\title{
Inconspicuous offender
}

Citation for published version (APA):

Bitorina, A. (2020). Inconspicuous offender: pathophysiological role of oxidized-low density lipoprotein in metabolic disease. [Doctoral Thesis, Maastricht University]. Maastricht University. https://doi.org/10.26481/dis.20200929ab

Document status and date:

Published: 01/01/2020

DOI:

10.26481/dis.20200929ab

Document Version:

Publisher's PDF, also known as Version of record

\section{Please check the document version of this publication:}

- A submitted manuscript is the version of the article upon submission and before peer-review. There can be important differences between the submitted version and the official published version of record.

People interested in the research are advised to contact the author for the final version of the publication, or visit the DOI to the publisher's website.

- The final author version and the galley proof are versions of the publication after peer review.

- The final published version features the final layout of the paper including the volume, issue and page numbers.

Link to publication

\footnotetext{
General rights rights.

- You may freely distribute the URL identifying the publication in the public portal. please follow below link for the End User Agreement:

www.umlib.nl/taverne-license

Take down policy

If you believe that this document breaches copyright please contact us at:

repository@maastrichtuniversity.nl

providing details and we will investigate your claim.
}

Copyright and moral rights for the publications made accessible in the public portal are retained by the authors and/or other copyright owners and it is a condition of accessing publications that users recognise and abide by the legal requirements associated with these

- Users may download and print one copy of any publication from the public portal for the purpose of private study or research.

- You may not further distribute the material or use it for any profit-making activity or commercial gain

If the publication is distributed under the terms of Article $25 \mathrm{fa}$ of the Dutch Copyright Act, indicated by the "Taverne" license above, 
Inconspicuous offender: Pathophysiological role of Oxidized-Low Density Lipoprotein in metabolic disease.

Albert Bitorina 


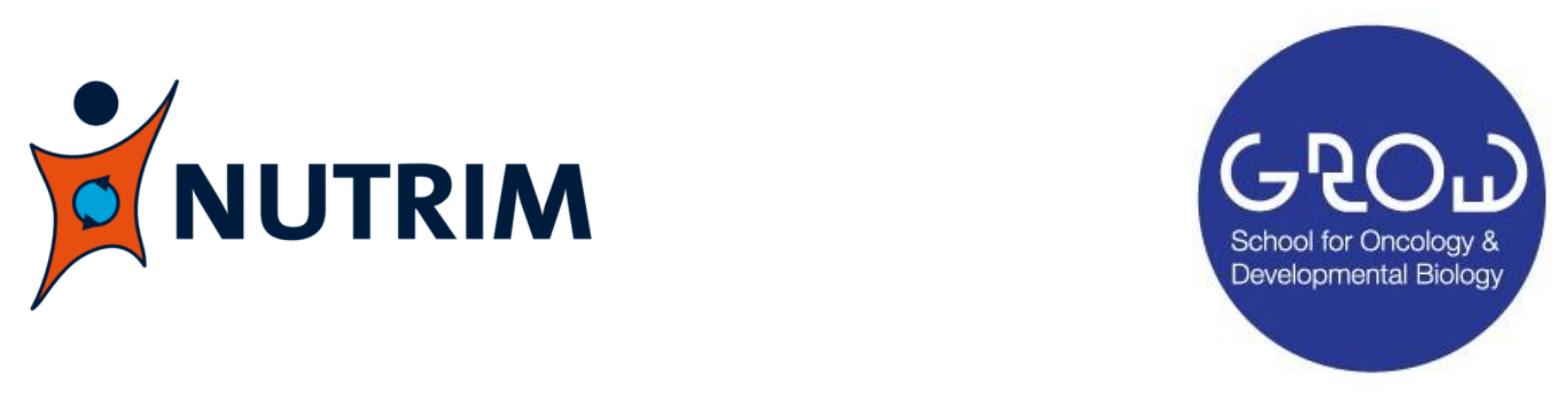

The studies presented in this thesis were performed within the NUTRIM (School of Nutrition and Translational Research in Metabolism) and GROW (School for Oncology \& Developmental Biology) at Maastricht University.

\section{Cover design: Albert Bitorina}

Layout: Albert Bitorina

Printed by: Gildeprint

ISBN: 9789464024722

(C) Copyright Albert Bitorina, Maastricht 2020

All rights reserved. No part of this thesis may be reproduced, distributed, or transmitted in any form or by any means, including photocopying, recording, or other electronic or mechanical methods, without the prior written permission of the author or publisher, except in the case of brief quotations embodied in critical reviews and certain other noncommercial uses permitted by copyright law. 


\title{
Inconspicuous offender: \\ Pathophysiological role of Oxidized-Low Density Lipoprotein in metabolic disease
}

\author{
DISSERTATION \\ to obtain the degree of Doctor at Maastricht University, \\ on the authority of the Rector Magnificus Prof. dr. Rianne M. Letschert, \\ in accordance with the decision of the Board of Deans,
} to be defended in public on Tuesday September 29, 2020, at 13.00 hours

by

Albert Bitorina 


\section{Promotor}

Prof. dr. R. Sverdlov

Dr. J. Theys

\section{Co-promotor}

Dr. Y. Oligschläger

\section{Assessment committee}

Prof. dr. J.F.C. Glatz (Chairman)

Prof. dr. J. Swinnen (KU Leuven, Belgium)

Prof. dr. S.E. Köhler

Prof. dr. N. Hellings (Hasselt University, Belgium)

Dr. A. Romano 


\section{Propositions}

Accompanying the dissertation

Inconspicuous offender:

\section{Pathophysiological role of Oxidized-Low Density Lipoprotein in metabolic disease}

1. Antibodies against oxLDL ameliorates the harmful consequences of disturbed lipid metabolism. (This Thesis)

2. Antibodies against oxLDL can improve outcomes of cancer therapy. (This Thesis)

3. OxLDL is one of the major drivers of the metabolic hallmark in cancer. (This Thesis)

4. The physiology of males and females are similar but not identical and so therapeutic strategies should be developed with this in mind. (This Thesis)

5. Current NASH and cancer therapeutic outcomes can also be improved by improving early diagnosis.

6. Science.... it's not a competition.

7. Science is pure so keep your politics, economics and non-scientific beliefs away from it unless you wish to taint it.

8. To ease the global burden of metabolic disease, oxLDL immunization should be utilized in the same manner as current standardized vaccines against infectious diseases.

9. The nature of man's life is to be lazy, the goal of innovation is to facilitate laziness, thus man works hard to innovate in order to achieve a lazy life.

10. What? Why? How? Yes, you must have the answer to all 3 for anything that you want to do.

11. "That's how they've always done it" leads to "that's how I've always done it" leads to "that's how we've always done it" leads to "I don't know why we've always done it this way" and so I say, one should never be content with "that's how we've always done it" as an explanation for anything.

Albert Bitorina

Maastricht - September $29^{\text {th }} 2020$ 


\section{Contents}

Chapter 1 - page 7 General introduction

Chapter 2 - page 17 Blood-derived macrophages prone to accumulate Lysosomal lipids trigger oxLDL-dependent murine hepatic inflammation

Chapter 3 - page 43 Cathepsin D regulates lipid metabolism in murine steatohepatitis

Chapter 4-page 77 Sex-opposed inflammatory effects of 27-hydroxycholesterol are mediated via estrogen signaling

Chapter 5 - page 111 Low profile high value target: the role of OxLDL in cancer

Chapter 6 - page 135 OxLDL as an inducer of a metabolic shift in cancer cells

Chapter 7 - page 155 Pneumococcal immunization reduces tumor burden and growth in a NASHHCC model: preliminary data from a pilot study

Chapter 8 - page 169 General Discussion

\section{Appendices}

page 178 Summary

page 180 Valorization 


\section{Chapter 1}

General introduction 


\section{Chapter 1}

\section{Chapter 1 General introduction}

\section{Lipids}

Lipids are small hydrophobic or amphiphilic molecules that are vital for many biological functions. Due to their amphiphilic properties, phospholipids serve as structural building blocks for cellular membranes and are capable of forming transport vesicles. Additionally, lipids can serve as signaling molecules in various cell signaling pathways and they are used as fuel for the cell due to their high amount of contained energy $(1,2)$.

Lipids such as cholesterol are important components of cell membranes but are also used as intermediate factors in signaling or digestion such as hormones (e.g. estrogen) or bile acids (e.g. 27hydroxycholesterol) (3). Other lipids such as triglycerides are primarily used as fuel due to the high energy content of their long chain fatty acids (1).

\section{Lipid trafficking}

Lipids are either obtained through the diet or synthesized de novo within tissues (Figure 1) (4). When gained through the diet lipids are first packaged into large transport vesicles known as chylomicrons and absorbed by the gut into the bloodstream before they are ultimately taken up by the liver. In the liver de novo synthesized and dietary lipids are either stored or processed further into cholesterol and triglycerides containing Very Low-Density Lipoprotein (VLDL) particles. These VLDL particles then carry cholesterol and triglycerides to extrahepatic tissues. While doing so they shed triglycerides which are subsequently taken up by adipose tissues. As the amount of triglycerides drops compared to the cholesterol present within the VLDL particle it is then referred to as Low Density Lipoprotein (LDL) (5). LDL is then taken up by extrahepatic tissues using the LDL membrane receptor which recognizes the apolipoprotein B-100 that is imbedded in the membrane of LDL (6). The LDL particle is subsequently processed in the lysosomes of the cell which are organelles with low internal $\mathrm{pH}$ containing several hydrolytic enzymes (e.g. lipase, Cathepsin D) that degrade many types of biomolecules such as lipids or proteins (7-9). Once broken down by the lysosome, cholesterol and fatty acids are then transported out of the lysosome through specialized transporter proteins such as NiemannPick type C1(10). Free cholesterol and fatty acids can subsequently be used by the cell to build membranes or as signaling molecules. In addition, they can be stored as reserve or be further broken down as a source of energy in the mitochondria. Metabolites resulting from the breakdown of cholesterol such as the bile acid precursor $27-\mathrm{HC}$ as well as excess free cholesterol can also be transferred out of the cell through the ATP Binding Cassette-transporters (ABC-transporters) and by means of High-Density Lipoprotein (HDL) particles be transported back to the liver for disposal through the bile $(11,12)$. 


\section{Chapter 1}

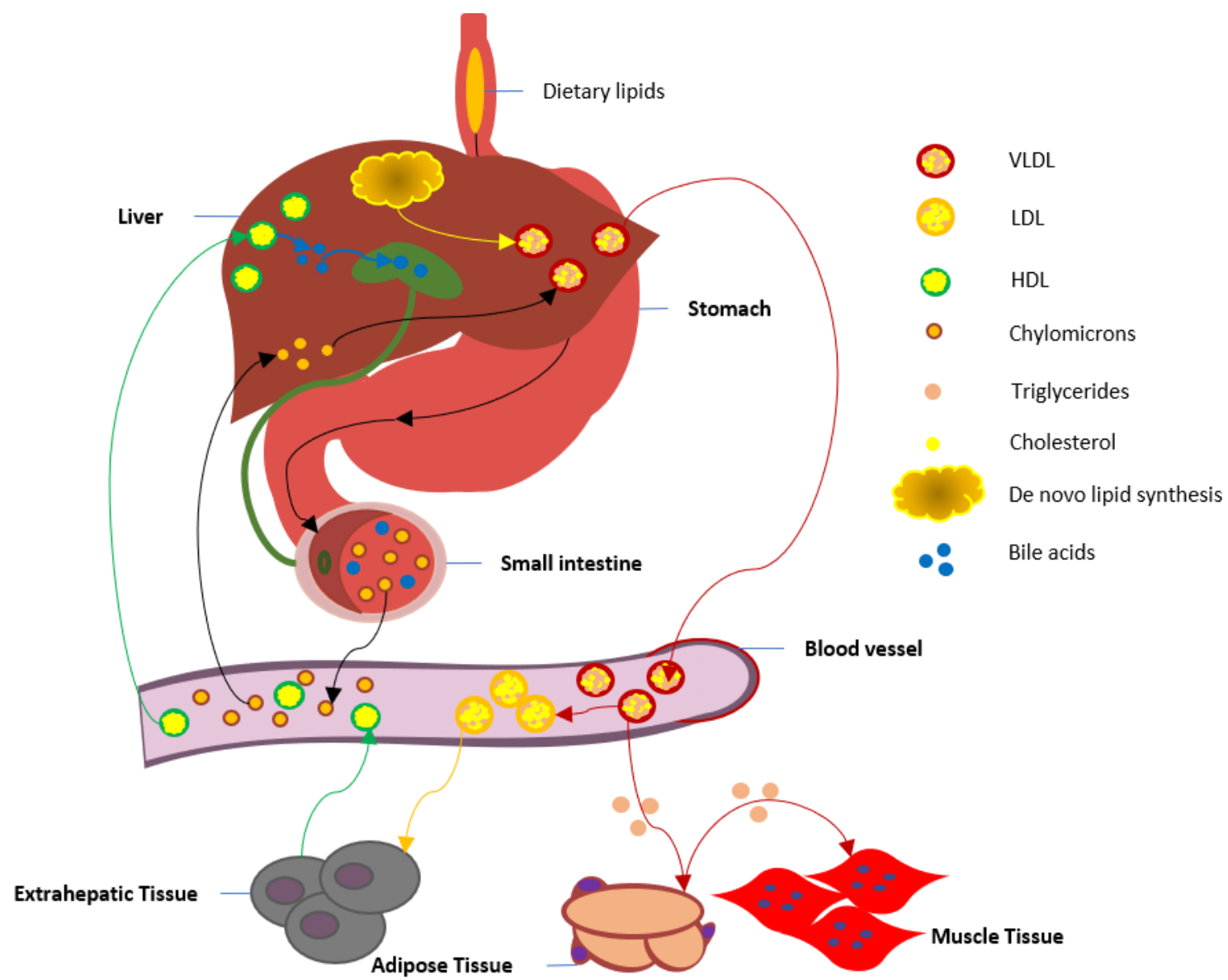

Figure 1: Lipid trafficking

\section{Oxidized LDL}

As lipids are vital to the normal functioning of cells, disturbances of their metabolic processing (due to excessive intake through the diet, impaired motility due to genetic deficiencies or structural changes), can lead to improper functioning of the cell and consequently to diseases development. Alterations to lipids through oxidation leads to poor cellular function and disease. The oxidation of lipids can occur in situations of increased oxidative stress (13). Oxidative stress results from an out-of-balance production of reactive oxygen species (ROS), which are by-products of cellular metabolism, generated in particular by mitochondrial respiration (14). Normal cellular levels of ROS are known to play an active role in modulating a variety of cell signaling pathways that for example govern growth, angiogenesis and metabolism. Whereas low physiological levels of ROS are preserved through active scavenging by antioxidants, high levels of ROS, which occur if the production of ROS exceeds the scavenging capacity of antioxidants, result in oxidative stress. Oxidative stress leads to damage to several cellular macromolecules such as proteins, DNA and lipids $(15,16)$. In case of the latter, changes can be induced in lipids carried by circulating lipoproteins as well as in the lipoproteins. Through this process of exposure to ROS, low-density lipoproteins (LDL) can become susceptible to oxidative 


\section{Chapter 1}

modifications, for instance in plasma or when retained in the artery wall, leading to conversion of LDL into oxLDL (Figure 2) (17). Once LDL is converted into oxLDl it is no longer recognized by the LDL receptor but is instead taken up by LOX-1, Cluster of differentiation 36 (CD36) or other scavenger receptors due to modifications to the B-100 protein (18). Increased levels of oxLDL are strongly correlated to various (metabolic) diseases.

\section{Low-Density Lipoprotein}

\section{Oxidized Low-Density Lipoprotein}

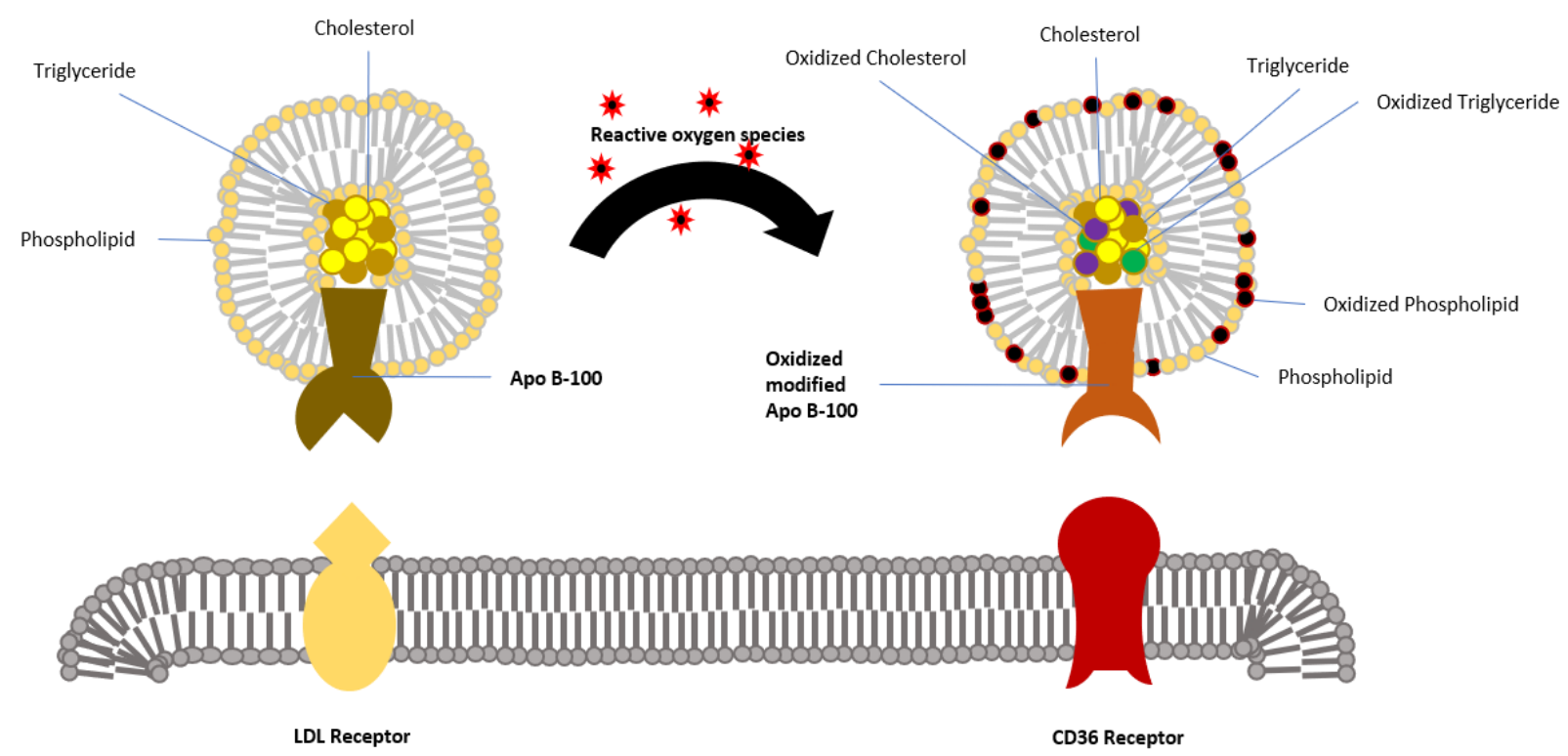

Figure 2: oxLDL formation

\section{7-hydroxycholesterol}

While lipid oxidation can take place in the plasma as exemplified with oxLDL, oxidized lipids also occur intracellularly with some of them performing various actions including cell signaling. One of these intracellularly modified lipids is 27-hydroxycholesterol $(27 \mathrm{HC})$, an oxysterol that plays an important role in the bile acid synthesis pathway and is the most abundant of all oxysterols found in the circulation. $27 \mathrm{HC}$ is produced by the mitochondrial enzyme cyp27a1 in extrahepatic tissues (19). In the liver $27 \mathrm{HC}$ is converted into 7 - $\alpha$-hydroxycholesterol $(7 \alpha \mathrm{HC})$ by cypb1 leading to production of chenodeoxycholic acid (CDCA) (20). While high concentrations of $27 \mathrm{HC}$ are found in foamy macrophages and in atherosclerotic plaques, it is reduced in npc1 mutant fibroblasts $(19,21)$. We have previously shown that $27 \mathrm{HC}$ can reduce both lysosomal cholesterol and cholesterol crystals in macrophages potentially leading to reduction of hepatic inflammation in LDLR ko mice (22). In addition, $27 \mathrm{HC}$ is known to act as a selective estrogen receptor modulator (SERM) (23). The mechanism of how $27 \mathrm{HC}$ modulates inflammation and how estrogen receptors play into this is not yet known. 


\section{Chapter 1}

\section{Cathepsin D}

Oxidized lipids such as oxLDL are known to negatively impact proper functioning of the lysosome and its components (24). The lysosome is an important cellular component involved in metabolic processes. Such process includes breakdown of nutrients into usable metabolites and building blocks for the cell. These breakdown processes are mediated by various enzyme families among which are the cathepsins (9). Cathepsin D is an aspartic protease synthesized in the rough endoplasmic reticulum and is thereafter transferred to the lysosomes. Cathepsin D activity leads to the metabolic breakdown of intracellular proteins, cell signaling and apoptosis. While cathepsin D is mostly active in the acidic milieu of the lysosomes, it is known to be secreted into the extracellular space in certain pathological conditions (25). For instance, changes in the lysosomal $\mathrm{pH}$ or accumulation of poorly degradable lipids such as oxLDL in the lysosomes are known to release cathepsin D into the circulation (26). Relevantly, it has been shown that extracellular cathepsin D activity is not only associated with metabolic-inflammatory disorders such as atherosclerosis and NAFLD but also cancer $(25,27)$. Cathepsin D is known to be associated with breast cancer and is thought to contribute to malignancy through breakdown of extracellular matrix thereby facilitating metastasis and increase proliferation through its indirect mitogenic effects $(28,29)$.

\section{Nonalcoholic steatohepatitis and oxLDL}

Among diseases commonly associated with dysregulated lipid metabolism is nonalcoholic steatohepatitis (NASH). Similar to nonalcoholic fatty liver disease (NAFLD) NASH is characterized by accumulation of lipids in the liver. However, unlike NAFLD, in NASH intrahepatic lipid accumulation is accompanied by intrahepatic inflammation, other complications associated with NASH are cirrhosis and hepatocellular carcinoma (HCC). Currently, the prevalence of NAFLD is estimated to be $25 \%$ world-wide, predominantly in the West, with NASH making up 5\% of the world population $(30,31)$. Clinicians face major challenges in diagnosing and treating NASH as the exact underlying mechanisms triggering hepatic inflammation are still largely unknown. We have previously demonstrated an association between hepatic inflammation and lysosomal lipid accumulation inside resident Kupffer cells (KCs) and in blood-derived macrophages $(22,32,33)$. This observation was confirmed by the presence of cholesterol-loaded KCs in the livers of NASH patients, strengthening the notion that lysosomal lipid storage in hepatic macrophages may be a potential mechanism for NASH (34). In line with our data, others have shown that incubating macrophages with oxLDL in vitro resulted in the accumulation of cholesteryl esters (CEs) and free cholesterol in the lysosome, which suggests the possibility of oxLDL being directly involved in lysosomal lipid-induced inflammation $(35,36)$. Furthermore, our previous data also suggests that lysosomal enzymes such as cathepsin D strongly correlate with lipid-induced inflammation in NASH. Here intracellular accumulation of lipids including 


\section{Chapter 1}

oxLDL are thought to destabilize lysosomal membranes leading to secretion of lysosomal enzymes such as cathepsin D into the plasma which are then thought to contribute to inflammation through unknown pathways (37).

\section{Niemann-Pick type C1 and oxLDL}

Niemann-Pick type C1 (NPC1), is a rare genetic disorder in which the function of the lysosomal membrane protein NPC1 is compromised, making it unable to shuttle cholesterol out of the lysosome. Due to a mutation in this gene, the intracellular trafficking of cholesterol is impaired leading to serious complications such as liver failure, splenomegaly, impairment of motor and cognitive functions all resulting in early death. While lysosomal lipid accumulation has been identified to be the main contributor to the NPC1 disease, other factors such as oxidative stress, apoptosis and inflammation have all been shown to be present and also contribute to disease progression (38). In line, cholesterol oxidation products which are enriched in oxLDL, are increased in plasma of in NPC1 disease patients. In a previous study, we studied the contribution of oxLDL to the disease progression of NPC1 by administering a vaccine to increase anti-oxLDL IgM autoantibodies in an NPC1 disease mouse model. The results showed improvement of systemic and neurological symptoms in vaccinated mice as seen from improved hepatic phenotype, including reduced liver lipid accumulation and inflammation as well as delayed regression of motor skills, reduced neuroinflammation and improved cerebellar phenotype compared to control-treated mice (38). Overall, our data indicate that targeting oxLDL is a promising approach to treat dyslipidemia related diseases.

\section{Cancer and oxLDL}

Cancer is characterized by abnormal uncontrolled cell growth along with the potential to spread to other distant tissues and is among the highest occurring diseases worldwide with 18 million new cases in 2018 alone (39). Dysregulated lipid metabolism is among the many risk factors for cancer development. High plasma lipids or metabolic diseases with hyperlipidemia such as obesity and NASH are all found to be correlated to increased cancer incidence. Due to this connection between lipids and cancer, there has been an increasing number of studies and clinical trials looking into the targeting of lipid metabolism as a means of preventing or treating cancer in recent years. Importantly, oxLDL has also been implicated in many aspects of cancer ranging from involvement in carcinogenesis due to its damaging effects on DNA, cancer progression by increasing cell proliferation or migration and cancer therapy where limited data point to its ability to reduce treatment efficacy (39). 


\section{Chapter 1}

\section{Thesis aim and outline}

In this thesis, we aimed to explore the role of oxidized lipids with a primary focus on oxLDL in the pathophysiology of metabolic diseases such as NASH, NPC1 and cancer. A variety of in vitro, in vivo and clinical study methods were employed to assess the disease progression markers affected by oxLDL or derivatives thereof such as inflammation, metabolism and response to treatment. In addition, we also sought to validate the use of antibodies against oxLDL as a promising readily-available therapeutic tool to prevent the detrimental effects of oxLDL.

Chapter 2 explores the direct effects of oxLDL on the inflammatory status of macrophages, which are thought to be the main drivers of inflammation in diseases featuring dyslipidemia, as well as verifying the ability of anti-oxLDL antibodies to prevent these effects. Chapter 3, is an in vitro study aimed at evaluating the effects of cathepsin D (CTSD) inhibition on the oxLDL mediated modulation of inflammation in macrophages in order to explore its therapeutic viability. Chapter 4 covers a novel attribute of a promising oxidized lipid (27-hydroxycholesterol) with lipid lowering therapeutic potential that shows different effects in men versus women. The current state of our understanding of the effects of oxLDl in cancer is laid out in chapter 5 and an in vitro study covered in chapter 6 aimed to further elucidate the metabolic effects of oxLDL in cancer. In addition, chapter 7 showcases preliminary results of ongoing in vivo studies looking into therapeutic potential of targeting oxLDL through immunization in a cancer model of NASH-derived hepatocellular carcinoma. Finally, chapter 8 discusses the overall conclusions of this thesis and addresses open questions that can be further investigated in future research in this context. 


\section{Chapter 1}

\section{References}

1. de Carvalho, C., and Caramujo, M. J. (2018) The Various Roles of Fatty Acids. Molecules 23

2. Muro, E., Atilla-Gokcumen, G. E., and Eggert, U. S. (2014) Lipids in cell biology: how can we understand them better? Mol Biol Cell 25, 1819-1823

3. Gill, S., Chow, R., and Brown, A. J. (2008) Sterol regulators of cholesterol homeostasis and beyond: the oxysterol hypothesis revisited and revised. Prog Lipid Res 47, 391-404

4. Stellaard, F., and Lütjohann, D. (2017) The Interpretation of Cholesterol Balance Derived Synthesis Data and Surrogate Noncholesterol Plasma Markers for Cholesterol Synthesis under Lipid Lowering Therapies. Cholesterol 2017, 5046294-5046294

5. Huang, L.-H., Elvington, A., and Randolph, G. J. (2015) The role of the lymphatic system in cholesterol transport. Front Pharmacol 6, 182-182

6. Sakamoto, N., and Rosenberg, A. S. (2011) Apolipoprotein B Binding Domains: Evidence That They Are Cell-Penetrating Peptides That Efficiently Deliver Antigenic Peptide for Cross-Presentation of Cytotoxic T Cells. The Journal of Immunology 186, 5004

7. Mulder, M., Lombardi, P., Jansen, H., van Berkel, T. J., Frants, R. R., and Havekes, L. M. (1993) Low density lipoprotein receptor internalizes low density and very low density lipoproteins that are bound to heparan sulfate proteoglycans via lipoprotein lipase. J Biol Chem 268, 9369-9375

8. Infante, R. E., and Radhakrishnan, A. (2017) Continuous transport of a small fraction of plasma membrane cholesterol to endoplasmic reticulum regulates total cellular cholesterol. Elife 6

9. Schulze, H., Kolter, T., and Sandhoff, K. (2009) Principles of lysosomal membrane degradation: Cellular topology and biochemistry of lysosomal lipid degradation. Biochim Biophys Acta 1793, 674-683

10. Thelen, A. M., and Zoncu, R. (2017) Emerging Roles for the Lysosome in Lipid Metabolism. Trends Cell Biol 27, 833-850

11. Westerterp, M., Bochem, A. E., Yvan-Charvet, L., Murphy, A. J., Wang, N., and Tall, A. R. (2014) ATP-binding cassette transporters, atherosclerosis, and inflammation. Circ Res 114, $157-170$

12. Röhrl, C., and Stangl, H. (2013) HDL endocytosis and resecretion. Biochimica et biophysica acta $1831,1626-1633$

13. Ito, F., Sono, Y., and Ito, T. (2019) Measurement and Clinical Significance of Lipid Peroxidation as a Biomarker of Oxidative Stress: Oxidative Stress in Diabetes, Atherosclerosis, and Chronic Inflammation. Antioxidants (Basel) 8, 72

14. Zorov, D. B., Juhaszova, M., and Sollott, S. J. (2014) Mitochondrial reactive oxygen species (ROS) and ROS-induced ROS release. Physiol Rev 94, 909-950

15. Lobo, V., Patil, A., Phatak, A., and Chandra, N. (2010) Free radicals, antioxidants and functional foods: Impact on human health. Pharmacogn Rev 4, 118-126 


\section{Chapter 1}

16. Schieber, M., and Chandel, N. S. (2014) ROS function in redox signaling and oxidative stress. Curr Biol 24, R453-R462

17. Lu, M., and Gursky, O. (2013) Aggregation and fusion of low-density lipoproteins in vivo and in vitro. Biomol Concepts 4, 501-518

18. Gleissner, C. A., Leitinger, N., and Ley, K. (2007) Effects of native and modified low-density lipoproteins on monocyte recruitment in atherosclerosis. Hypertension 50, 276-283

19. Brown, A. J., and Jessup, W. (1999) Oxysterols and atherosclerosis. Atherosclerosis 142, 1-

28

20. Fuchs, M. (2003) Bile acid regulation of hepatic physiology: III. Regulation of bile acid synthesis: past progress and future challenges. Am J Physiol Gastrointest Liver Physiol 284, G551-557

21. Frolov, A., Zielinski, S. E., Crowley, J. R., Dudley-Rucker, N., Schaffer, J. E., and Ory, D. S. (2003) NPC1 and NPC2 regulate cellular cholesterol homeostasis through generation of low density lipoprotein cholesterol-derived oxysterols. J Biol Chem 278, 25517-25525

22. Bieghs, V., Hendrikx, T., Gorp, P., Verheyen, F., Guichot, Y., Walenbergh, S., Gijbels, M., Rensen, S., Bast, A., Plat, J., Kalhan, S., Hofker, M., Lütjohann, D., and Shiri-Sverdlov, R. (2012) The Cholesterol Derivative 27-Hydroxycholesterol Reduces Steatohepatitis in Mice. Gastroenterology 144

23. Umetani, M., and Shaul, P. W. (2011) 27-Hydroxycholesterol: the first identified endogenous SERM. Trends Endocrinol Metab 22, 130-135

24. Cox, B. E., Griffin, E. E., Ullery, J. C., and Jerome, W. G. (2007) Effects of cellular cholesterol loading on macrophage foam cell lysosome acidification. J Lipid Res 48, 10121021

25. Benes, P., Vetvicka, V., and Fusek, M. (2008) Cathepsin D--many functions of one aspartic protease. Crit Rev Oncol Hematol 68, 12-28

26. Hoppe, G., O'Neil, J., Hoff, H. F., and Sears, J. (2004) Products of lipid peroxidation induce missorting of the principal lysosomal protease in retinal pigment epithelium. Biochim Biophys Acta 1689, 33-41

27. Khurana, P., Yadati, T., Goyal, S., Dolas, A., Houben, T., Oligschlaeger, Y., Agarwal, A. K., Kulkarni, A., and Shiri-Sverdlov, R. (2019) Inhibiting Extracellular Cathepsin D Reduces Hepatic Steatosis in Sprague-Dawley Rats (†). Biomolecules 9, 171

28. Dian, D., Vrekoussis, T., Shabani, N., Mylonas, I., Kuhn, C., Schindlbeck, C., Navrozoglou, I., Friese, K., Makrigiannakis, A., and Jeschke, U. (2012) Expression of cathepsin-D in primary breast cancer and corresponding local recurrence or metastasis: an immunohistochemical study. Anticancer Res 32, 901-905

29. Tan, G.-J., Peng, Z.-K., Lu, J.-P., and Tang, F.-Q. (2013) Cathepsins mediate tumor metastasis. World J Biol Chem 4, 91-101

30. Araújo, A. R., Rosso, N., Bedogni, G., Tiribelli, C., and Bellentani, S. (2018) Global epidemiology of non-alcoholic fatty liver disease/non-alcoholic steatohepatitis: What we need in the future. Liver Int 38 Suppl 1, 47-51 


\section{Chapter 1}

31. Povsic, M., Wong, O. Y., Perry, R., and Bottomley, J. (2019) A Structured Literature Review of the Epidemiology and Disease Burden of Non-Alcoholic Steatohepatitis (NASH). Adv Ther 36, 1574-1594

32. Bieghs, V., Walenbergh, S. M. A., Hendrikx, T., van Gorp, P. J., Verheyen, F., Olde Damink, S. W., Masclee, A. A., Koek, G. H., Hofker, M. H., Binder, C. J., and Shiri-Sverdlov, R. (2013) Trapping of oxidized LDL in lysosomes of Kupffer cells is a trigger for hepatic inflammation. Liver international : official journal of the International Association for the Study of the Liver 33, 1056-1061

33. Bieghs, V., van Gorp, P. J., Walenbergh, S. M. A., Gijbels, M. J., Verheyen, F., Buurman, W. A., Briles, D. E., Hofker, M. H., Binder, C. J., and Shiri-Sverdlov, R. (2012) Specific immunization strategies against oxidized low-density lipoprotein: a novel way to reduce nonalcoholic steatohepatitis in mice. Hepatology 56, 894-903

34. Ioannou, G. N., Haigh, W. G., Thorning, D., and Savard, C. (2013) Hepatic cholesterol crystals and crown-like structures distinguish NASH from simple steatosis. J Lipid Res 54, 1326-1334

35. Yancey, P. G., and Jerome, W. G. (2001) Lysosomal cholesterol derived from mildly oxidized low density lipoprotein is resistant to efflux. J Lipid Res 42, 317-327

36. Ullery-Ricewick, J. C., Cox, B. E., Griffin, E. E., and Jerome, W. G. (2009) Triglyceride alters lysosomal cholesterol ester metabolism in cholesteryl ester-laden macrophage foam cells. J Lipid Res 50, 2014-2026

37. Walenbergh, S. M. A., Houben, T., Rensen, S. S., Bieghs, V., Hendrikx, T., van Gorp, P. J., Oligschlaeger, Y., Jeurissen, M. L. J., Gijbels, M. J. J., Buurman, W. A., Vreugdenhil, A. C. E., Greve, J. W. M., Plat, J., Hofker, M. H., Kalhan, S., Pihlajamäki, J., Lindsey, P., Koek, G. H., and Shiri-Sverdlov, R. (2016) Plasma cathepsin D correlates with histological classifications of fatty liver disease in adults and responds to intervention. Sci Rep 6, 3827838278

38. Houben, T., Magro dos Reis, I., Oligschlaeger, Y., Steinbusch, H., Gijbels, M. J. J., Hendrikx, T., Binder, C. J., Cassiman, D., Westerterp, M., Prickaerts, J., and Shiri-Sverdlov, R. (2019) Pneumococcal Immunization Reduces Neurological and Hepatic Symptoms in a Mouse Model for Niemann-Pick Type C1 Disease. Frontiers in Immunology 9

39. Bitorina, A. V., Oligschlaeger, Y., Shiri-Sverdlov, R., and Theys, J. (2019) Low profile high value target: The role of OxLDL in cancer. Biochim Biophys Acta Mol Cell Biol Lipids 1864, 158518 


\section{Chapter 2}

Blood-derived macrophages prone to accumulate Lysosomal lipids trigger oxLDLdependent murine hepatic inflammation 
Chapter 2 


\section{Chapter 2}

Chapter 2 Blood-derived macrophages prone to accumulate Lysosomal lipids trigger oxLDL-dependent murine hepatic inflammation

Tom Houben, Yvonne Oligschlaeger, Albert V Bitorina, Tim Hendrikx, Sofie MA Walenbergh, Marie-Hélène Lenders, Marion JJ Gijbels, Fons Verheyen, Dieter Lütjohann, Marten H Hofker, Christoph J Binder, Ronit Shiri-Sverdlov

*A.V.B contributed by assisting with the acquisition of data; (statistical) analysis and interpretation of data; revising the paper critically and gave final approval of the version to be submitted.

Sci Rep. 2017 Oct 2;7(1):12550 


\title{
Chapter 2
}

\begin{abstract}
Despite the consistent rise of non-alcoholic steatohepatitis (NASH) worldwide, the mechanisms that govern the inflammatory aspect of this disease remain unknown. Previous research showed an association between hepatic inflammation and lysosomal lipid accumulation in blood-derived hepatic macrophages. Additionally, in vitro findings indicated that lipids, specifically derived from the oxidized low-density lipoprotein (oxLDL) particle, are resistant to removal from lysosomes. On this basis, we investigated whether lysosomal lipid accumulation in blood-derived hepatic macrophages is causally linked to hepatic inflammation and assessed to what extent increasing anti-oxLDL IgM autoantibodies can affect this mechanism. By creating a proof-of-concept mouse model, we demonstrate a causal role for lysosomal lipids in blood-derived hepatic macrophages in mediating hepatic inflammation and initiation of fibrosis. Furthermore, our findings show that increasing anti-oxLDL IgM autoantibody levels reduces inflammation. Hence, therapies aimed at improving lipid-induced lysosomal dysfunction and blocking oxLDL-formation deserve further investigation in the context of NASH.
\end{abstract}




\section{Chapter 2}

\section{Introduction}

Analogous to the steep rise of obesity and diabetes, the prevalence of non-alcoholic fatty liver disease (NAFLD) is currently estimated at $25 \%$ to $45 \%$ in the general population 1,2 . NAFLD encompasses a spectrum of liver diseases that are histologically categorized in nonalcoholic fatty liver (NAFL) and non-alcoholic steatohepatitis (NASH) 3. Whereas hepatic steatosis without hepatic injury is referred to as NAFL, NASH is defined by a conjunction of steatosis and inflammation, which presents with or without fibrosis 3 . Though the development of inflammation paves the way for advanced liver diseases, the mechanisms underlying the hepatic inflammatory response are largely unknown. As this lack of mechanistic understanding is a key antecedent for the lack of well-defined effective therapies, it is of utmost importance to improve the knowledge regarding the mechanisms triggering hepatic inflammation.

Previous research from our group indicated an association between hepatic inflammation and lysosomal lipid accumulation inside resident Kupffer cells (KCs) as well as in blood-derived macrophages4-6. This relation was confirmed by the presence of cholesterol-accumulating KCs in livers of NASH patients, bolstering the notion of lysosomal lipid storage in hepatic macrophages as a potential mechanism for NASH 7. Also, it has been shown that incubating macrophages with oxLDL in vitro results in the lysosomal accumulation of cholesteryl esters (CEs) and free cholesterol, suggesting a direct role for oxLDL in mediating lysosomal lipid-induced inflammation 8,9. Of note, excessive accumulation of lipids in the lysosomal compartment of cells also occurs in the context of NiemannPick type C1 (NPC1) disease. Characterized by hepatosplenomegaly, foam cell formation and hepatic inflammation, NPC1 patients exhibit features resembling NASH 10. Therefore, it becomes evident that the pathology of lysosomal lipid accumulation is not limited to lysosomal lipid storage diseases such as NPC1, but also plays a role in other lipid-associated inflammatory diseases such as NASH.

Previously, it has been demonstrated that oxLDL and the bacterium Streptococcus pneumoniae exhibit molecular mimicry for the phosphorylcholine (PC) epitope, a major target for naturally occurring immunoglobulin M (IgM) antibodies. Immunizing mice with heat-killed Streptococcus pneumoniae has been shown to increase anti-oxLDL IgM autoantibodies and to reduce atherosclerotic lesion formation 11 and hepatic inflammation 5 in low-density lipoprotein receptor knockout (Ldlr-/-) mice. Unlike acetylated LDL (acLDL) and native LDL, injection of oxLDL in hyperlipidemic mice showed increased lysosomal lipid accumulation in hepatic macrophages, corroborating the detrimental effect of oxLDL in promoting inflammation by disturbing the physiology of the lysosome 6. Altogether, several studies indicate that accumulation of oxLDL and associated lipids in lysosomes of macrophages is associated with increased inflammation. However, none of these studies provide specific evidence that lysosomal 


\section{Chapter 2}

storage of lipids in hepatic macrophages can be a mechanistic trigger for inflammation in NASH. Moreover, to what extent oxLDL contributes to lysosomal lipid accumulation-induced hepatic inflammation has to our knowledge never been investigated.

Here, we investigated whether lysosomal lipid accumulation in blood-derived hepatic macrophages is a mechanistic trigger for hepatic inflammation and assessed to what extent anti-oxLDL IgM autoantibodies can affect this mechanism. For this purpose, lysosomal lipid accumulation in bloodderived hepatic macrophages was generated by transplanting bone marrow of Npc1-mutant (Npc1mut) or wildtype (Npc1wt) mice into Ldlr-/- mice on a high-fat, high-cholesterol (HFC) diet for 12 weeks. To investigate the specific contribution of oxLDL on lysosomal lipid-induced hepatic inflammation, mice were immunized with heat-killed Streptococcus pneumoniae 5,11. This study describes lysosomal lipid accumulation in blood-derived hepatic macrophages as a novel mechanism that triggers hepatic inflammation. Moreover, our findings suggest a role for oxLDL in mediating lysosomal lipid-induced hepatic inflammation. 


\section{Chapter 2}

\section{Methods}

\section{Mice, bone marrow transplantation, immunization, and diet}

Niemann-Pick type C1nih mutant (Npc1mut) mice (a kind gift from Prof. Dr. Lieberman from University of Michigan Medical School) were backcrossed into a C57BL/6 background for more than 10 generations. Npc1mut and Ldlr-/- mice were housed under standard conditions and had access to food and water ad libitum. Experiments were performed according to Dutch regulations and approved by the Committee for Animal Welfare of Maastricht University.

To generate myeloid Npc1mut deficient Ldlr-/- mice, bone marrow transplantations were performed. Twenty-two week-old female Ldlr-/- mice received antibiotic water containing neomycin $(100 \mathrm{mg} / \mathrm{l}$; Gibco, Breda, the Netherlands) and 6*104 U/l polymycin B sulphate (Gibco, Breda, the Netherlands) one week before and four weeks after irradiation. One day before and on the day of the transplantation, Ldlr-/- mice were lethally irradiated with 6 Gray of $\gamma$-radiation, thus receiving 12 Gray in total. Lethally irradiated Ldlr-/- mice were then injected with $1 * 107$ bone marrow cells donated from either Npc1mut mice or wildtype littermate controls (Npc1wt). In order to fully ensure bone marrow replacement, mice had a nine week recovery period. After nine weeks of recovery, transplanted (-tp) mice received a highfed, high-cholesterol (HFC) diet, containing $21 \%$ butter and $0.2 \%$ cholesterol (diet 1635; Scientific Animal Food and Engineering, Villemoissonsur-Orge, France) for 12 weeks. Five weeks after bone marrow transplantation, mice were divided into three groups. One group received the equivalent of 108 colony-forming units of heat-killed R36A (unencapsulated Streptococcus pneumoniae) emulsified in $200 \mu 1$ sterile $0.9 \% \mathrm{NaCl}$ for the primary subcutaneous immunization. Subsequently, two intraperitoneal booster immunizations were administered every two weeks. The two other control groups received an $0.9 \% \mathrm{NaCl}$ injection only. From the start of the diet, intraperitoneal booster immunizations were administered every three weeks. An overall overview of the experimental set-up is depicted in Supplementary Fig. S4 online.

Liver tissue was isolated and snap-frozen in liquid nitrogen and stored at $-80^{\circ} \mathrm{C}$ or fixed in $4 \%$ formaldehyde/PBS. The biochemical determination of plasma cholesterol and liver triglyceride levels, electron microscopy, RNA isolation, complementary DNA synthesis, quantitative polymerase chain reaction and auto-antibody titers against anti-oxLDL IgM antibodies are described extensively 4,5,4346. Liver cholesterol levels were quantified as described previously 47. 


\section{Chapter 2}

\section{Bone marrow-derived macrophages}

Bone marrow-derived macrophages (BMDMs) were isolated from the tibiae and femurs of wildtype and Npc1mut mice. Cells were cultured in RPMI-1640 (GIBCO Invitrogen, Breda, the Netherlands) with $10 \%$ heat-inactivated fetal calf serum (Bodinco B.V. Alkmaar, the Netherlands), penicillin (100 $\mathrm{U} / \mathrm{ml})$, streptomycin $(100 \mu \mathrm{g} / \mathrm{ml})$ and L-glutamine $2 \mathrm{mM}$ (all GIBCO Invitrogen, Breda, the Netherlands), supplemented with 20\% L929-conditioned medium (LCM) for 8-9 days to generate BMDMs. After attachment, Npc1mut macrophages were seeded at 350,000 cells per well in 24-well plates and incubated for $24 \mathrm{~h}$ with oxLDL ( $25 \mu \mathrm{g} / \mathrm{ml}$; Alfa Aesar: J65591, Wardhill, MA, USA), with or without anti-oxLDL EO6 antibodies (Avanti Polar Lipids, Alabaster, AL, USA). Wildtype macrophages underwent a similar procedure, but did not receive the EO6 antibodies. Then cells were washed and stimulated with lipopolysaccharide (LPS; $100 \mathrm{ng} / \mathrm{ml}$ ) for $3 \mathrm{~h}$ (wildtype cells) or $4 \mathrm{~h}$ (Npc1mut cells). Finally, supernatant was collected for protein measurements and cells were lysed for mRNA expression analysis.

\section{Statistical analysis}

Data were statistically analyzed by performing two-tailed non-paired t-tests using GraphPad Prism, version 6.0 for Windows. Data were expressed as the mean \pm SEM and considered significant at $\mathrm{p}<$ 0.05 . $* * *$ and $* * *$ indicate $\mathrm{p}<0.05,0.01$ and 0.001 respectively. Additional explanation is provided in Supplementary Information. 


\section{Chapter 2}

\section{Results}

Lysosomal lipid accumulation in blood-derived hepatic macrophages results in a severe hepatic pathological phenotype

To ensure successful bone marrow replacement, bone marrow efficiency was assessed. Transplantation of both wildtype and Npc1mut bone marrow approximated an efficiency of 90\% (Supplementary Table S1 online), proving the bone marrow transplantation successful.

Furthermore, to confirm the successful transplantation at the microscopic level, hepatic tissues were subjected to electron microscopy analysis. As pointed out by the lower magnification, livers from Npc1mut-tp mice demonstrated dense clusters of macrophages which resembled granuloma-like structures. These structures were absent in Npc1wt-tp mice, indicating that these structures are related to the NPC1 mutation (Fig. 1A). Also, the majority of resident KCs were located adjacent to the large granulomas, containing various numbers of small lipid inclusions (resembling cholesterol crystals (Supplementary Fig. S1 online)). No detectable differences in phenotype between non-immunized and immunized Npc1mut-tp mice were observed.
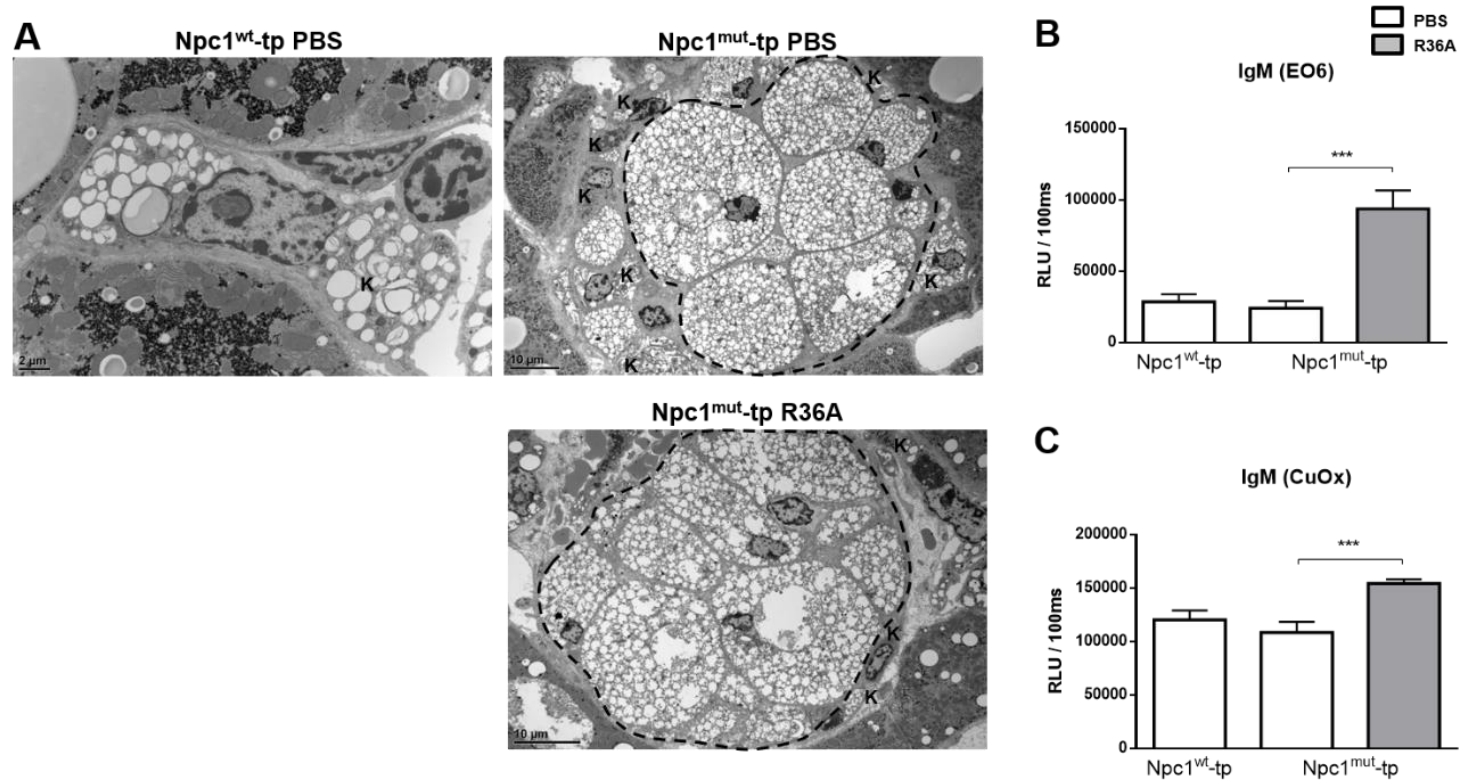

Figure 1: Hepatic phenotype of Npc1 wt-tp and Npc1 mut-tp mice and IgM autoantibody titers in plasma. (A) Representative electron microscopy pictures of resident (Kupffer cell) and bone marrow-derived macrophages of Npc1 wt-tp (scale bar $2 \mu \mathrm{m}$ ) and control or immunized Npc1 mut-tp mice (scale bar $10 \mu \mathrm{m}$ ). Area within the dashed line: Npc1 mut granuloma; K: Kupffer cell. (B,C) IgM EO6 antibodies (B) and IgM antibodies to copper-oxidized (CuOx)LDL (C) were measured in plasma of mice with or without immunization at a dilution of 1:100. Data are expressed as relative light units (RLU)/100 ms. $n=10-11$ mice/group. Asterisks indicate significant difference from nonimmunized Npc1 mut-tp mice by use of two-tailed unpaired $t$ test. ${ }^{* * *} \mathrm{p}<0.001$. All error bars are SEM. 


\section{Chapter 2}

Relevantly, these granuloma structures were also visible at higher microscopic magnification by $\mathrm{H} \& \mathrm{E}$ staining (Supplementary Fig. S2 online). Also, relative liver and spleen weights were dramatically increased in mice transplanted with Npc1mut bone marrow compared to Npc1wt-tp mice, confirming the severe pathological phenotype (Supplementary Fig. S2 online). After immunization, both relative liver and spleen weight decreased in immunized Npc1mut-tp compared to control-treated Npc1mut-tp, suggesting that inhibition of oxLDL uptake by macrophages ameliorates the pathological phenotype.

\section{Increased anti-oxLDL IgM autoantibody titers after heat-killed pneumococci immunization}

To determine whether immunization with heat-killed Streptococcus pneumoniae was performed successfully, IgM autoantibody levels were measured in the plasma. Immunization with heat-killed pneumococci resulted in an increase of plasma IgM antibodies of the EO6/T15 idiotype (Fig. 1B), which bind oxLDL by specifically recognizing the phosphorylcholine epitope 12. In line, increased IgM antibodies against copper-oxidized LDL (Cu-oxLDL) were detected in immunized mice compared to control mice (Fig. 1C). Thus, immunization with heat-killed pneumococci induced a modest antioxLDL IgM autoantibody production in Npc1mut-tp mice, confirming successfulness of the immunization.

\section{Disturbances in lipid metabolism are partly restored after elevation of anti-oxLDL IgM autoantibody levels}

To determine the effect of lysosomal lipid accumulation in hepatic macrophages on lipid metabolism, we examined cholesterol and triglyceride levels in liver and plasma. Whereas hepatic cholesterol levels were elevated in Npc1mut-tp mice compared to Npc1wt-tp mice, immunization of Npc1mut-tp mice decreased hepatic cholesterol, indicating improved hepatic cholesterol metabolism upon immunization (Fig. 2A). In contrast, plasma cholesterol levels reduced by almost 50\% in Npc1mut-tp mice compared to Npc1wt-tp mice, but did not differ between immunized and non-immunized Npc1mut-tp mice (Fig. 2B). Detailed investigation of the size of the hepatic granulomas by means of CD68 staining confirmed the presence of granulomas in Npc1mut-tp mice, while being absent in Npc1wt-tp mice (Fig. 2C-D). Additionally, immunizing Npc1mut-tp mice with heat-killed pneumococci resulted in a strong decrease of granuloma size, suggesting oxLDL as an important compound in disturbing cholesterol metabolism in this model (Fig. 2C-D). 


\section{Chapter 2}

A

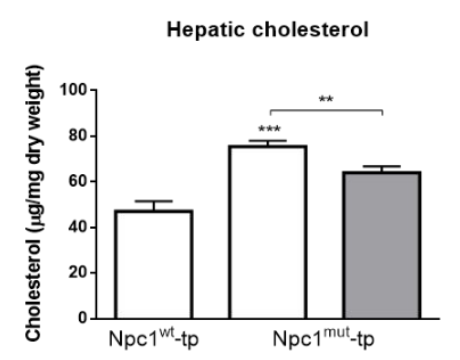

C

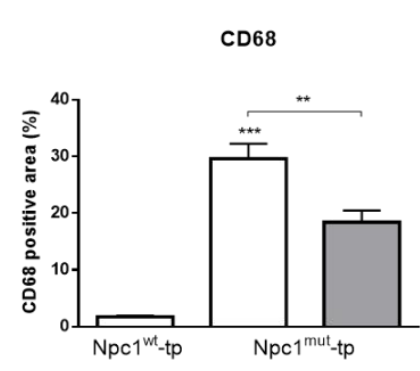

E

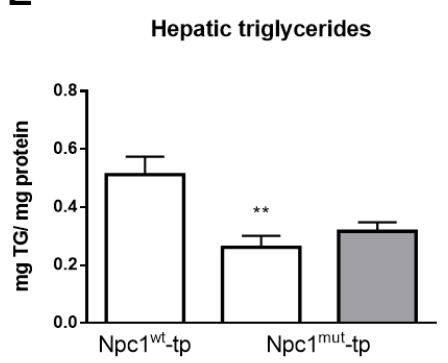

B
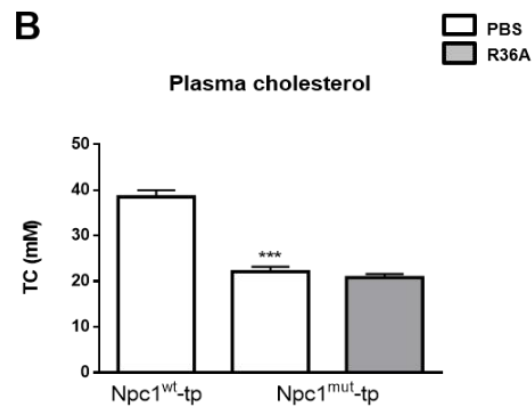

D

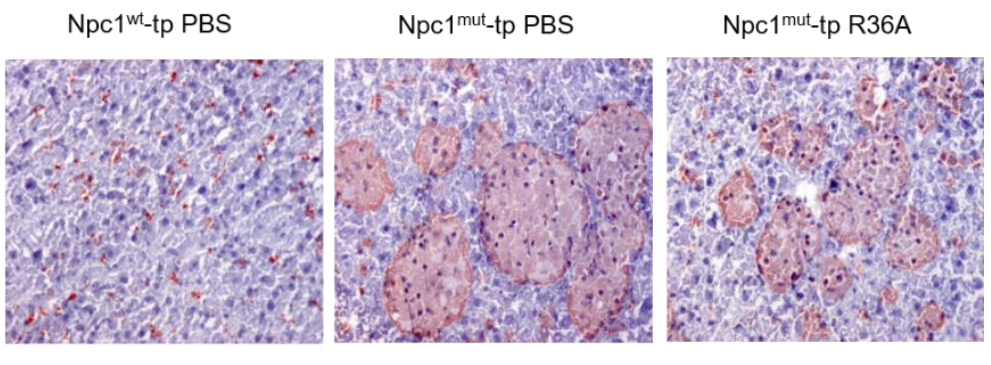

F

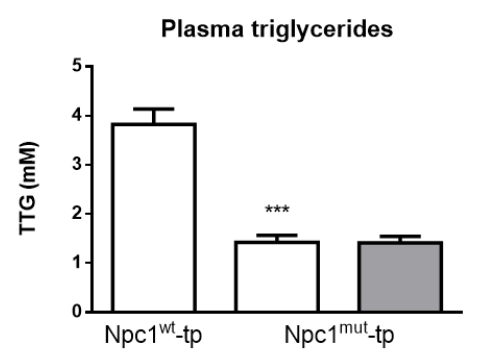

G

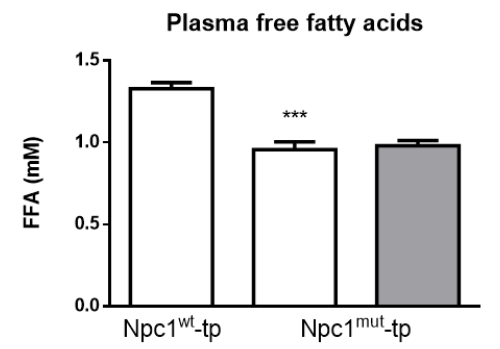

H

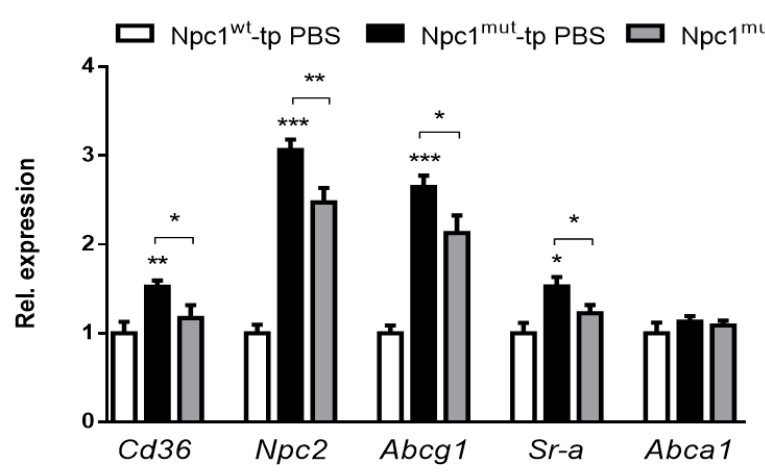

Figure 2: Lipid parameters. (A,B) Hepatic and plasma cholesterol levels of Npc1 wt-tp and control-treated or immunized Npc1 mut-tp mice on HFC diet. (C) Quantification of CD68 staining by measuring CD68 positive area. (D) Representative histological pictures of the CD68 staining (200x magnification). (E,F) Hepatic and plasma triglyceride levels. (G) Plasma free fatty acids. (H) Hepatic gene expression analysis of Cd36, Npc2 and Abcg1, Sr-a and Abca1. n =9-11 mice/group. Gene expression data are set relative to Npc1 wt-tp mice. Asterisks indicate significant difference from non-immunized Npc1 wt-tp and Npc1 mut-tp mice by use of two-tailed unpaired $\mathrm{t}$ test. *, ** and *** indicate $\mathrm{p}<0.05,0.01$ and 0.001 resp. All error bars are SEM. TC, total cholesterol; TTG, total triglycerides. 


\section{Chapter 2}

Furthermore, hepatic triglyceride levels were decreased in Npc1mut-tp mice compared to Npc1wt-tp mice and remained similar between immunized and control-treated Npc1mut-tp mice (Fig. 2E). Analogous to hepatic triglycerides, similar trends were observed in plasma triglyceride and free fatty acid levels (Fig. 2F-G).

To further define the influences on hepatic lipid metabolism, hepatic expression of genes involved in lipid homeostasis was examined. Compared to Npc1wt-tp mice, Npc1mut-tp mice showed increased gene expression levels of Cluster of differentiation 36 (Cd36), Scavenger receptor A (Sr-a), NiemannPick type $\mathrm{C} 2$ (Npc2) and ATP-binding cassette transporter G1 (Abcg1), confirming disturbance of lipid metabolism (Fig. 2H). Furthermore, though no differences were observed in gene expression levels of the ATP-binding cassette transporter A1 (Abca1) (Fig. 2H), expression levels of all other markers were reduced upon R36A immunization of Npc1mut-tp mice, strengthening the importance of oxLDL to the disturbances in lipid metabolism in this model. Altogether, these results imply an important contribution of oxLDL to lysosomal lipid-induced disturbances in lipid metabolism.

\section{OxLDL contributes to lysosomal lipid-induced hepatic inflammation}

To determine whether lysosomal lipid accumulation in blood-derived macrophages is a trigger for hepatic inflammation, hepatic cryosections were stained for the inflammatory markers Mac-1 (infiltrated macrophages and neutrophils; against Cd11b) and NIMP (neutrophils). Number of cells expressing both inflammatory markers was increased in Npc1mut-tp mice compared to Npc1wt-tp mice, supporting our hypothesis that lysosomal lipid accumulation in blood-derived macrophages is a direct trigger for hepatic inflammation (Fig. 3A-B). Additionally, increasing circulating anti-oxLDL IgM autoantibodies reduced Mac-1- and NIMP-positive cell levels (Fig. 3A-C). These inflammatory findings were confirmed by performing hepatic gene expression analysis of the inflammatory markers integrin alpha M (Itgam), tumor necrosis factor alpha (Tnf $\alpha$ ), interleukin 12 (Il12), CXC chemokine receptor-4 (Cxcr4), monocyte chemoattractant protein 1 (Mcp1), Caspase-1 and CC chemokine receptor-2 (Ccr2) (Fig. 3D). Relevantly, while single, just infiltrated macrophages were positive for Mac-1, macrophages present inside the large granulomas of the Npc1mut-tp group were Mac-1negative (Fig. 3C). This observation revealed that blood-derived hepatic macrophages lose the Cd11b phenotypic marker over time. 


\section{Chapter 2}

A
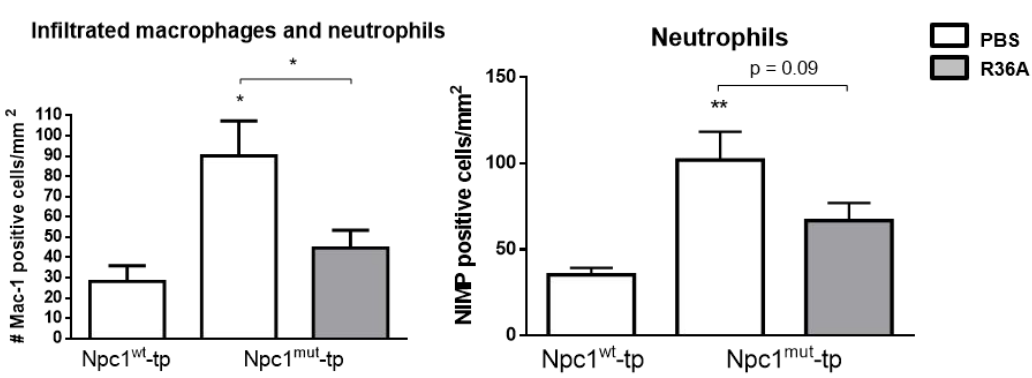

B

Npc1wt-tp PBS

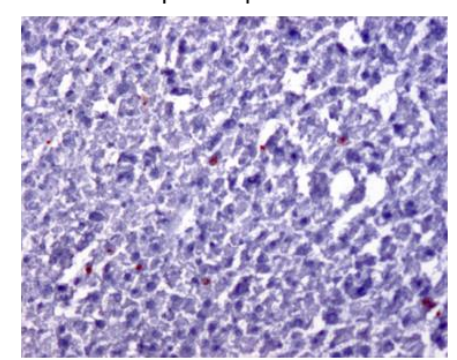

Npc1 ${ }^{\text {mut_tp PBS }}$

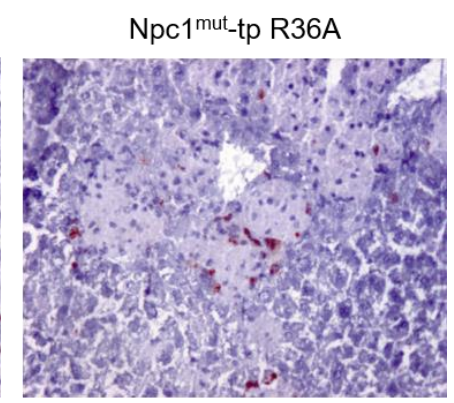

C

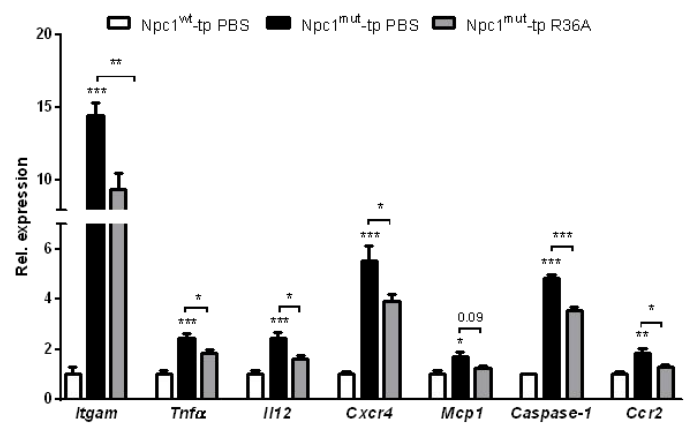

Figure 3: Parameters of hepatic inflammation. (A,B) Liver sections were stained for both infiltrating macrophages and neutrophils (Mac1) and neutrophils solely (NIMP) and counted. (C) Representative images of the Mac-1 staining (magnification $\times 200$ ) after HFC feeding of Npc1 wt-tp and control-treated or immunized Npc1 mut-tp mice for 12 weeks. (D) Hepatic gene expression analysis of Itgam, Tnfo, Il12, Cxcr4, Mcp1, Caspase-1 and Ccr2. Gene expression data were set relative to Npc1 wt-tp mice. $n=9-11$ mice/group. Asterisks indicate significant difference from non-immunized Npc1 wt-tp and Npc1 mut-tp mice by use of two-tailed unpaired $\mathrm{t}$ test. *p $<0.05 ;{ }^{* *} \mathrm{p}<0.01$; $* * * \mathrm{p}<0.001$. All error bars are SEM.

To confirm the pro-inflammatory properties of oxLDL in bone marrow-derived macrophages (BMDMs), wildtype BMDMs were incubated with oxLDL for 24 hours, followed by 3 hour stimulation with lipopolysaccharide (LPS). Gene expression levels of the pro-inflammatory markers Tnf $\alpha$ and Mcp1 were increased upon oxLDL incubation, confirming the pro-inflammatory effect of oxLDL in BMDMs (Supplementary Fig. S3 online). Next, to explore the specific contribution of anti-oxLDL IgM autoantibodies to lysosomal lipid-induced inflammation in blood-derived macrophages, we isolated Npc1mut BMDMs and stimulated these with oxLDL, in the absence or presence of the anti-oxLDL antibody EO6. In the presence of EO6 antibodies, Npc1mut BMDMs stimulated with oxLDL demonstrated reduced inflammation, as indicated by reduced TNF $\alpha$ protein levels and reduced Tnf $\alpha$ and Ccr2 gene expression (Fig. 4). 


\section{Chapter 2}

A

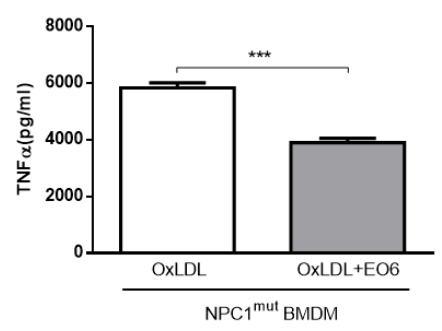

B

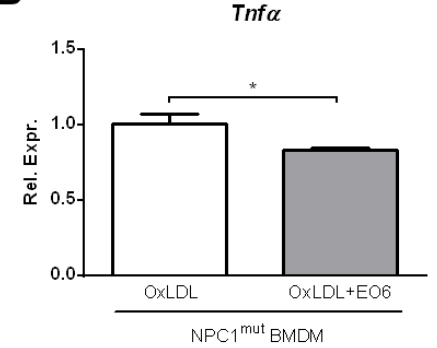

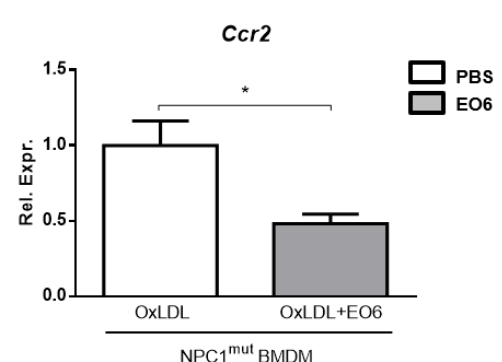

Figure 4: EO6-treatment reduced oxLDL-induced inflammation in Npc1 mut BMDMs. TNF $\alpha$ protein levels (A) and gene expression of inflammatory-related genes Tnf $\alpha$ and Ccr2 (B) after oxLDL loading of Npc1 mut BMDMs in absence or presence of anti-oxLDL EO6 antibodies. All data represent $\mathrm{n}=3$ (triplicates) for each experimental group. Asterisks indicate significant difference from non-immunized Npc1 wt-tp and Npc1 mut-tp mice by use of two-tailed unpaired t test. ${ }^{*} \mathrm{p}<0.05$; $* * * \mathrm{p}<0.001$. All error bars are SEM.

Combining the in vivo and in vitro data, these results reveal for the first time that lysosomal lipid accumulation in blood-derived macrophages is a mechanistic trigger for lipid-induced hepatic inflammation. On top, these data identify an essential role for oxLDL in mediating these inflammatory effects.

\section{Increasing anti-oxLDL IgM autoantibodies attenuates initiation of hepatic fibrosis in mice carrying lysosomal-lipid storing hepatic macrophages}

As hepatic fibrosis is a key symptom of active NASH 13, we investigated whether the inflammatory trigger of lysosomal lipid accumulation in blood-derived hepatic macrophages can initiate hepatic fibrosis. Hepatic collagen levels, characterized by Sirius Red staining, were elevated in Npc1mut-tp mice compared to Npc1wt-tp mice (Fig. 5A-B), as indicated by the increased collagen formation surrounding the granulomas (Fig. 5B). R36A immunization of Npc1mut-tp mice showed a trend towards a decrease in the level of fibrosis (Fig. 5A). Furthermore, these histological findings were confirmed by gene expression levels of the fibrotic markers, transforming growth factor beta (Tgf- $\beta$ ) and tissue inhibitor of metalloproteinase-3 (Timp3) (Fig. 5C). Altogether, these data suggest that oxLDL can initiate hepatic fibrosis by contributing to lysosomal lipid accumulation in blood-derived hepatic macrophages. 


\section{Chapter 2}

\section{A 吕 ${ }^{\text {PBs }}$}

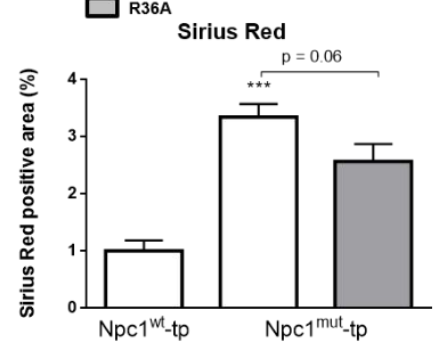

c

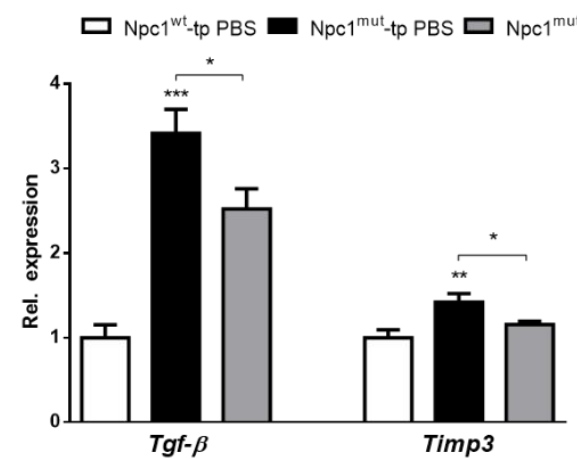

Figure 5: Parameters of hepatic fibrosis. (A) Quantification of Sirius Red (collagen) staining. (B) Representative pictures of Sirius Red staining (original magnification, 100x) of Npc1 wt-tp mice and Npc1 mut-tp mice with or without immunization on an HFC diet for 3 months. (C) Gene expression analysis of the fibrosis markers, Tgf- $\beta$ and Timp3. $n=9-11$ mice/group. Gene expression data are shown relative to Npc1 wt-tp mice by use of two-tailed unpaired t test. ${ }^{*} \mathrm{p}<0.05 ; * * \mathrm{p}<0.01 ; * * * \mathrm{p}<0.001$. All error bars are SEM. 


\section{Chapter 2}

\section{Discussion}

Currently, the mechanisms underlying NASH are poorly understood, restricting the development of well-defined, effective therapies. Previously, an association was demonstrated between murine hepatic inflammation and lysosomal lipid accumulation in hepatic macrophages4-6. Here, we prove that lysosomal lipid accumulation in blood-derived hepatic macrophages is a direct trigger for hepatic inflammation and initiates fibrosis. Moreover, our results show that increasing anti-oxLDL IgM autoantibody levels improves inflammation and lipid metabolism, suggesting that oxLDL plays a key role in mediating hepatic inflammation by promoting lipid accumulation in lysosomes of blood-derived hepatic macrophages. Therefore, this study provides additional insights into the inflammatory mechanisms driving NASH. Therapies aimed at improving lipid-induced lysosomal dysfunction and blocking the formation of oxLDL should therefore be investigated in the future.

Whereas initially viewed as the cell's degradation center, it has become increasingly clear that the lysosome constitutes a central role in regulating a plethora of physiological processes 14 . Specifically, upon accumulation of macromolecules (including lipids), lysosomes gradually lose their proteolytic and regulatory function, leading to disturbances in processes related to apoptosis, autophagy, calcium homeostasis, protein folding and, above all, metabolism and inflammation 15. Indeed, severely increased inflammation was observed in NPC1 16 and Wolman 17 disease patients, both lysosomal lipid storage disorders (LSDs), thereby linking lipid storage to inflammation. Likewise, previous studies by us and others have demonstrated an association between hepatic inflammation and lysosomal lipid accumulation 5,6,18. Building on this previous knowledge, we here demonstrate for the first time that lysosomal lipid storage in blood-derived hepatic macrophages can be viewed as an actual trigger for hepatic inflammation and initiates fibrosis, two central features of NASH. Our data also imply that oxLDL contributes to this hepatic inflammatory mechanism. As such, we propose that lysosomal accumulation of oxLDL-derived lipids is a trigger for NASH.

The observation that elevations in anti-oxLDL IgM autoantibody levels could partly protect from the inflammatory phenotype observed in Npc1mut-tp Ldlr-/- mice suggests that an important fraction of lysosomal lipids in blood-derived hepatic macrophages originates from oxLDL particles. In line, while circulating levels of oxLDL were reported to represent $0.001 \%$ of native LDL in healthy individuals 19, oxLDL levels were shown to increase up to $1.8 \%$ in patients with the metabolic syndrome 20 . Relevantly, 7 $\beta$-hydroxycholesterol and 7-ketocholesterol, the main cholesterol oxidation products present inside oxLDL particles 21,22, were previously linked to inflammatory processes, suggesting that cholesterol oxidation products contained in the oxLDL particle are responsible for eliciting inflammation 23,24. Also, oxLDL-induced formation of cholesterol crystals was demonstrated to 


\section{Chapter 2}

induce lysosomal membrane permeabilization 25-27. Lysosomal membrane permeabilization and the subsequent release of lysosomal enzymes into the cytoplasm is a known prerequisite for activation of the inflammasome 28, increased apoptosis 29 and of necrotic cell death 30, three pathways leading to increased hepatic inflammation. In line with these observations, blood-derived hepatic Npc1mut macrophages exhibited ruptured membrane structures, suggesting that cholesterol oxidation products induced lysosomal permeabilization in these macrophages resulting in hepatic inflammation. However, more evidence is needed to prove the actual contribution of cholesterol oxidation products to lysosomal permeabilization. Furthermore, to what extent oxLDL levels in our mouse model are comparable to oxLDL levels in NASH patients is not completely clear. Nevertheless, as both NASH patients 31 and Npc1-/--tp Ldlr-/- mice (described by Zhang et al. 32) show increased levels of plasma oxidized lipids, our mouse model can be considered a proper model to investigate the contribution of oxidized lipids to the human NASH situation. Oxidation of lipids were also shown to occur within lysosomes 33 . Therefore, it is likely that the immunized Npc1mut-tp group still contains internally oxidized lipid products within lysosomes, which contribute to the hepatic inflammatory response.

Besides cholesterol oxidation products, other components present inside or in the surface monolayer of oxLDL particles have also been associated with inflammatory responses. For example, oxidized phopspholipids and their highly reactive degradation product malondialdehyde, two lipid products identified on the surface of the oxLDL particle 34,35, have been identified in NASH patients 31,36,37. Furthermore, other lipids such as free cholesterol, cholesteryl esters, proteins and their oxidized derivatives have been identified in oxLDL 24. Additionally, while next to oxidized lipids also other non-oxidized cholesterol products contribute to the observed pathology, the finding that targeting specifically oxidized LDL leads to significant improvements in liver pathology provides evidence for an essential role for oxLDL in mediating lysosomal lipid-induced hepatic inflammation. Therefore, future research should aim to identify and provide detailed knowledge of the lipids specifically contained in oxLDL particles as this might lead to the identification of novel therapeutic targets for NASH.

Of note, besides oxLDL, also apoptotic cells expose the PC-epitope which is recognized by IgM autoantibodies described in this manuscript 38. As increased apoptosis has often been associated with increased levels of hepatic inflammation 39,40, it is possible that elevations in IgM autoantibody levels reduced hepatic inflammation in our model via increased clearance of apoptotic cells, rather than preventing the uptake of oxLDL by hepatic macrophages. 


\section{Chapter 2}

The current study also describes that blood-derived hepatic macrophages, which exhibited lysosomal lipid accumulation due to a NPC1 mutation, lose the CD11b phenotypic marker (indicative for hematopoietic origin 41) and gain the CD68 phenotypic marker (indicative for resident KCs 41) after infiltration in the liver, suggesting that blood-derived macrophages have phenotypically switched into resident KCs. Additionally, embryonically-derived resident KCs displayed intracellular cholesterol crystal formation and engulfed the granulomas (which appeared to be the result of a clustering of bloodderived hepatic macrophages carrying the NPC1 mutation). Therefore, the inability to eliminate the inflammatory stimulus (which is derived from the granulomas created by blood-derived hepatic macrophages) in the liver is likely due to a combination of dysfunctional blood-derived hepatic macrophages as well as the lack of a functional repertoire of resident embryonically-derived KCs to overcome this insult. Indeed, under inflammatory conditions, blood-derived tissue-resident macrophages were shown to dominate the inflammatory response in tissues 42 . Therefore, the findings of the current study indicate that functional blood-derived hepatic macrophages are essential to overcome lipid challenges in the liver and suggest that improving the lysosomal function in macrophages (from hematopoietic or embryonic origin) can be beneficial for NASH.

Although a bone marrow-specific NPC1 mutation was used in this study, the phenomenon of lysosomal lipid accumulation in hepatic macrophages in NASH patients is not necessarily the result of a deficiency of the Npc1 gene, but rather a consequence of a prolonged exposure of lipids to the liver. Furthermore, patients suffering from NASH traditionally show increased hepatic levels of cholesterol and triglycerides 2, while our model only showed elevations in hepatic cholesterol levels. Therefore, the mouse model described in this study should be considered a proof-of-concept mouse model for the involvement of lysosomal lipid storage in blood-derived hepatic macrophages to hepatic inflammation and fibrosis rather than a mouse model exactly mimicking the human situation of NASH.

\section{Conclusions}

In conclusion, we demonstrate here for the first time that lipid accumulation in lysosomes of bloodderived hepatic macrophages is a key trigger of hepatic inflammation and mediates initiation of fibrosis. Furthermore, increasing anti-oxLDL IgM autoantibody levels ameliorated the pathological phenotype, suggesting a key role for oxLDL in this process. Hence, therapies aimed at improving lipid-induced lysosomal dysfunction and blocking the formation of oxLDL should be further investigated in the context of NASH and might be of relevance for other metabolic inflammatory disorders. 


\section{Chapter 2}

\section{References}

1 Williams, C. D. et al. Prevalence of nonalcoholic fatty liver disease and nonalcoholic steatohepatitis among a largely middle-aged population utilizing ultrasound and liver biopsy: a prospective study. Gastroenterology 140, 124-131 (2011).

Rinella, M. E. Nonalcoholic fatty liver disease: a systematic review. JAMA 313, 2263-2273, doi:10.1001/jama.2015.5370 (2015).

3 Chalasani, N. et al. The diagnosis and management of non-alcoholic fatty liver disease: practice Guideline by the American Association for the Study of Liver Diseases, American College of Gastroenterology, and the American Gastroenterological Association. Hepatology 55, 2005 2023, doi:10.1002/hep.25762 (2012).

4 Bieghs, V. et al. The cholesterol derivative 27-hydroxycholesterol reduces steatohepatitis in mice. Gastroenterology 144, 167-178 e161 (2013).

5 Bieghs, V. et al. Specific immunization strategies against oxidized low-density lipoprotein: a novel way to reduce nonalcoholic steatohepatitis in mice. Hepatology 56, 894-903 (2012).

6 Bieghs, V. et al. Trapping of oxidized LDL in lysosomes of Kupffer cells is a trigger for hepatic inflammation. Liver Int 33, 1056-1061 (2013).

7 Ioannou, G. N., Haigh, W. G., Thorning, D. \& Savard, C. Hepatic cholesterol crystals and crown-like structures distinguish NASH from simple steatosis. J Lipid Res 54, 1326-1334 (2013).

8 Yancey, P. G. \& Jerome, W. G. Lysosomal cholesterol derived from mildly oxidized low density lipoprotein is resistant to efflux. J Lipid Res 42, 317-327 (2001).

9 Ullery-Ricewick, J. C., Cox, B. E., Griffin, E. E. \& Jerome, W. G. Triglyceride alters lysosomal cholesterol ester metabolism in cholesteryl ester-laden macrophage foam cells. J Lipid Res 50, 2014-2026, doi:10.1194/jlr.M800659-JLR200 (2009).

10 Patterson, M. C. et al. Disease and patient characteristics in NP-C patients: findings from an international disease registry. Orphanet J Rare Dis 8, 12, doi:10.1186/1750-1172-8-12 (2013).

11 Binder, C. J. et al. Pneumococcal vaccination decreases atherosclerotic lesion formation: molecular mimicry between Streptococcus pneumoniae and oxidized LDL. Nat Med 9, 736743 (2003).

12 Shaw, P. X. et al. Natural antibodies with the T15 idiotype may act in atherosclerosis, apoptotic clearance, and protective immunity. The Journal of clinical investigation 105, 1731-1740 (2000). 


\section{Chapter 2}

13 Angulo, P. et al. Liver Fibrosis, but No Other Histologic Features, Is Associated With Longterm Outcomes of Patients With Nonalcoholic Fatty Liver Disease. Gastroenterology 149, 389397 e310, doi:10.1053/j.gastro.2015.04.043 (2015).

14 Xu, H. \& Ren, D. Lysosomal physiology. Annu Rev Physiol 77, 57-80, doi:10.1146/annurevphysiol-021014-071649 (2015).

15 Platt, F. M., Boland, B. \& van der Spoel, A. C. The cell biology of disease: lysosomal storage disorders: the cellular impact of lysosomal dysfunction. J Cell Biol 199, 723-734, doi:10.1083/jcb.201208152 (2012).

16 Cologna, S. M. et al. Human and mouse neuroinflammation markers in Niemann-Pick disease, type C1. J Inherit Metab Dis 37, 83-92, doi:10.1007/s10545-013-9610-6 (2014).

17 Bernstein, D. L., Hulkova, H., Bialer, M. G. \& Desnick, R. J. Cholesteryl ester storage disease: review of the findings in 135 reported patients with an underdiagnosed disease. J Hepatol 58, 1230-1243, doi:10.1016/j.jhep.2013.02.014 (2013).

18 Yan, C. et al. Macrophage-specific expression of human lysosomal acid lipase corrects inflammation and pathogenic phenotypes in lal-/- mice. The American journal of pathology 169, 916-926 (2006).

19 Shoji, T. et al. Inverse relationship between circulating oxidized low density lipoprotein (oxLDL) and anti-oxLDL antibody levels in healthy subjects. Atherosclerosis 148, 171-177 (2000).

20 Holvoet, P. et al. The metabolic syndrome, circulating oxidized LDL, and risk of myocardial infarction in well-functioning elderly people in the health, aging, and body composition cohort. Diabetes 53, 1068-1073 (2004).

21 Hulten, L. M. et al. Oxysterols present in atherosclerotic tissue decrease the expression of lipoprotein lipase messenger RNA in human monocyte-derived macrophages. The Journal of clinical investigation 97, 461-468, doi:10.1172/JCI118436 (1996).

22 Brown, A. J., Dean, R. T. \& Jessup, W. Free and esterified oxysterol: formation during copperoxidation of low density lipoprotein and uptake by macrophages. J Lipid Res 37, 320-335 (1996).

23 Poli, G., Biasi, F. \& Leonarduzzi, G. Oxysterols in the pathogenesis of major chronic diseases. Redox Biol 1, 125-130, doi:10.1016/j.redox.2012.12.001 (2013).

24 Parthasarathy, S., Raghavamenon, A., Garelnabi, M. O. \& Santanam, N. Oxidized low-density lipoprotein. Methods Mol Biol 610, 403-417, doi:10.1007/978-1-60327-029-8_24 (2010). 


\section{Chapter 2}

25 Duewell, P. et al. NLRP3 inflammasomes are required for atherogenesis and activated by cholesterol crystals. Nature 464, 1357-1361, doi:10.1038/nature08938 (2010).

$26 \mathrm{Li}, \mathrm{W}$. et al. Cell death induced by 7-oxysterols via lysosomal and mitochondrial pathways is p53-dependent. Free radical biology \& medicine 53, 2054-2061, doi:10.1016/j.freeradbiomed.2012.09.007 (2012).

27 Hendrikx, T., Walenbergh, S. M., Hofker, M. H. \& Shiri-Sverdlov, R. Lysosomal cholesterol accumulation: driver on the road to inflammation during atherosclerosis and non-alcoholic steatohepatitis. Obes Rev 15, 424-433, doi:10.1111/obr.12159 (2014).

28 Hornung, V. et al. Silica crystals and aluminum salts activate the NALP3 inflammasome through phagosomal destabilization. Nat Immunol 9, 847-856, doi:10.1038/ni.1631 (2008).

29 Boya, P. \& Kroemer, G. Lysosomal membrane permeabilization in cell death. Oncogene 27, 6434-6451, doi:10.1038/onc.2008.310 (2008).

30 Lima, H., Jr. et al. Role of lysosome rupture in controlling Nlrp3 signaling and necrotic cell death. Cell Cycle 12, 1868-1878, doi:10.4161/cc.24903 (2013).

31 Chalasani, N., Deeg, M. A. \& Crabb, D. W. Systemic levels of lipid peroxidation and its metabolic and dietary correlates in patients with nonalcoholic steatohepatitis. The American journal of gastroenterology 99, 1497-1502, doi:10.1111/j.1572-0241.2004.30159.x (2004).

32 Zhang, J. R. et al. Niemann-Pick $\mathrm{C} 1$ protects against atherosclerosis in mice via regulation of macrophage intracellular cholesterol trafficking. The Journal of clinical investigation 118, 2281-2290, doi:10.1172/JCI32561 (2008).

33 Wen, Y. \& Leake, D. S. Low density lipoprotein undergoes oxidation within lysosomes in cells. Circ Res 100, 1337-1343, doi:10.1161/CIRCRESAHA.107.151704 (2007).

34 Miller, Y. I. et al. Oxidation-specific epitopes are danger-associated molecular patterns recognized by pattern recognition receptors of innate immunity. Circ Res 108, 235-248, doi:10.1161/CIRCRESAHA.110.223875 (2011).

35 Binder, C. J., Papac-Milicevic, N. \& Witztum, J. L. Innate sensing of oxidation-specific epitopes in health and disease. Nat Rev Immunol 16, 485-497, doi:10.1038/nri.2016.63 (2016).

36 Ikura, Y. et al. Localization of oxidized phosphatidylcholine in nonalcoholic fatty liver disease: impact on disease progression. Hepatology 43, 506-514, doi:10.1002/hep.21070 (2006).

37 Yesilova, Z. et al. Systemic markers of lipid peroxidation and antioxidants in patients with nonalcoholic Fatty liver disease. The American journal of gastroenterology 100, 850-855, doi:10.1111/j.1572-0241.2005.41500.x (2005). 


\section{Chapter 2}

38 Binder, C. J. et al. Innate and acquired immunity in atherogenesis. Nat Med 8, 1218-1226, doi:10.1038/nm1102-1218 (2002).

39 Feldstein, A. E. et al. Hepatocyte apoptosis and fas expression are prominent features of human nonalcoholic steatohepatitis. Gastroenterology 125, 437-443 (2003).

40 Tamimi, T. I. et al. An apoptosis panel for nonalcoholic steatohepatitis diagnosis. J Hepatol 54, 1224-1229, doi:10.1016/j.jhep.2010.08.023 (2011).

41 Davies, L. C., Jenkins, S. J., Allen, J. E. \& Taylor, P. R. Tissue-resident macrophages. Nat Immunol 14, 986-995, doi:10.1038/ni.2705 (2013).

42 Yona, S. et al. Fate mapping reveals origins and dynamics of monocytes and tissue macrophages under homeostasis. Immunity 38, 79-91, doi:10.1016/j.immuni.2012.12.001 (2013).

43 Bieghs, V. et al. Internalization of modified lipids by CD36 and SR-A leads to hepatic inflammation and lysosomal cholesterol storage in Kupffer cells. PloS one 7, e34378 (2012).

44 Bieghs, V. et al. Role of scavenger receptor A and CD36 in diet-induced nonalcoholic steatohepatitis in hyperlipidemic mice. Gastroenterology 138, 2477-2486, 2486 e2471-2473 (2010).

45 Wouters, K. et al. Dietary cholesterol, rather than liver steatosis, leads to hepatic inflammation in hyperlipidemic mouse models of nonalcoholic steatohepatitis. Hepatology 48, 474-486 (2008).

46 Wisse, E. et al. Fixation methods for electron microscopy of human and other liver. World J Gastroenterol 16, 2851-2866 (2010).

47 Lutjohann, D. et al. High doses of simvastatin, pravastatin, and cholesterol reduce brain cholesterol synthesis in guinea pigs. Steroids 69, 431-438 (2004). 


\section{Chapter 2}

\section{Supplemental Figures}

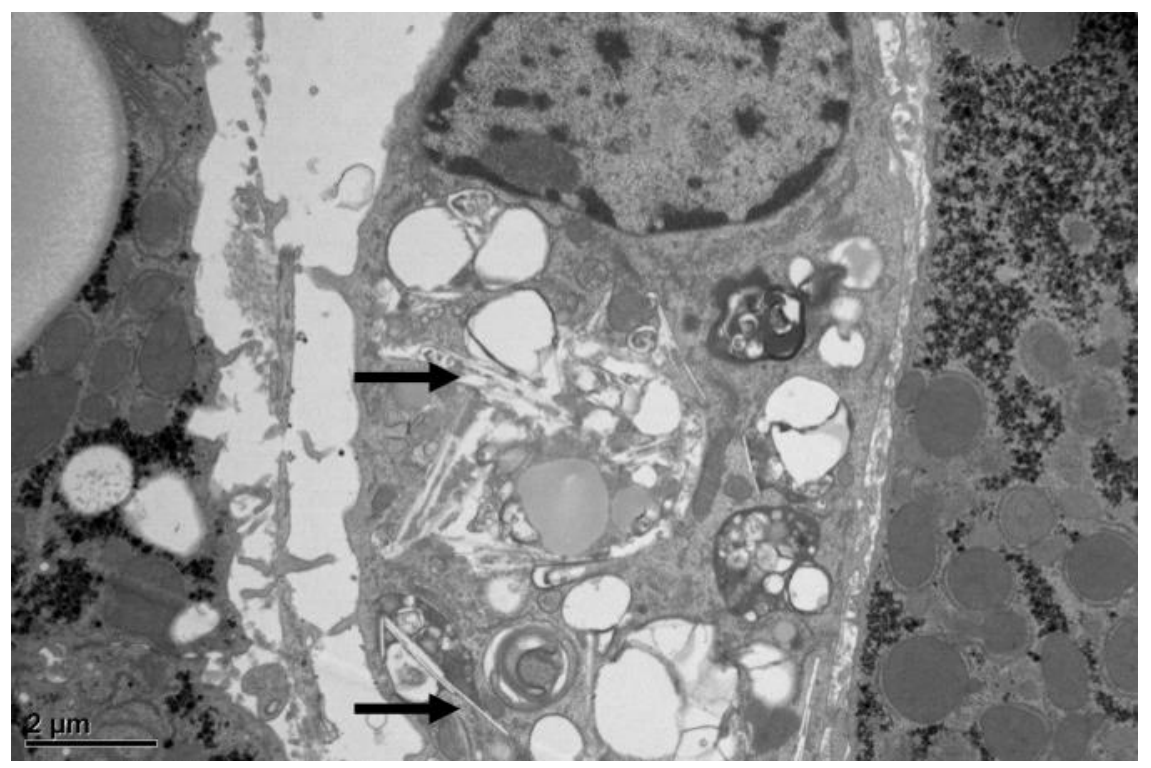

Supplemental Figure 1: Hepatic phenotype of Npc1mut-tp mice. The formation of cholesterol crystals was observed in Kupffer cells (indicated by the arrows) that engulfed the granuloma-like structures in Npc1mut-tp mice. 


\section{Chapter 2}

A

Npc1wt-tp PBS

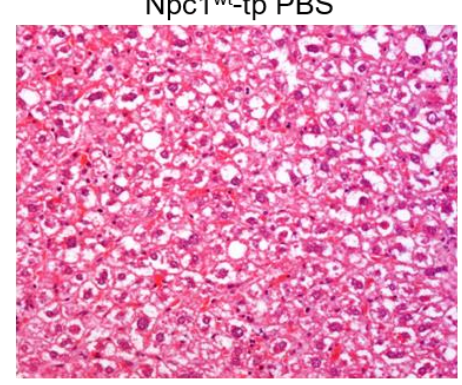

Npc1 ${ }^{\text {mut_tp }}$ PBS

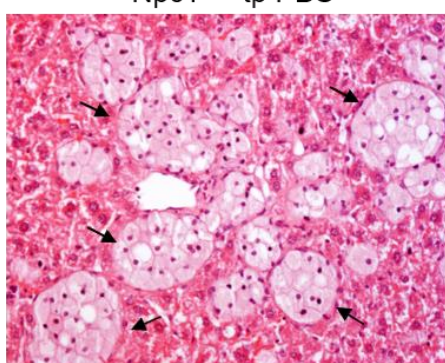

Npc1 $1^{\text {mut-tp R36A }}$

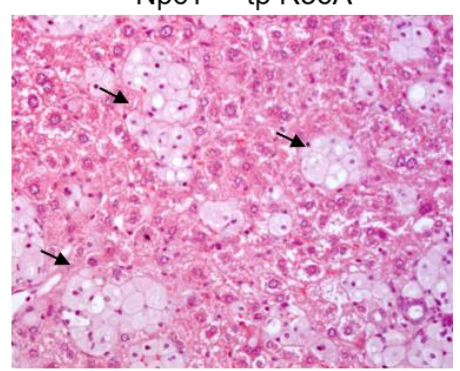

B

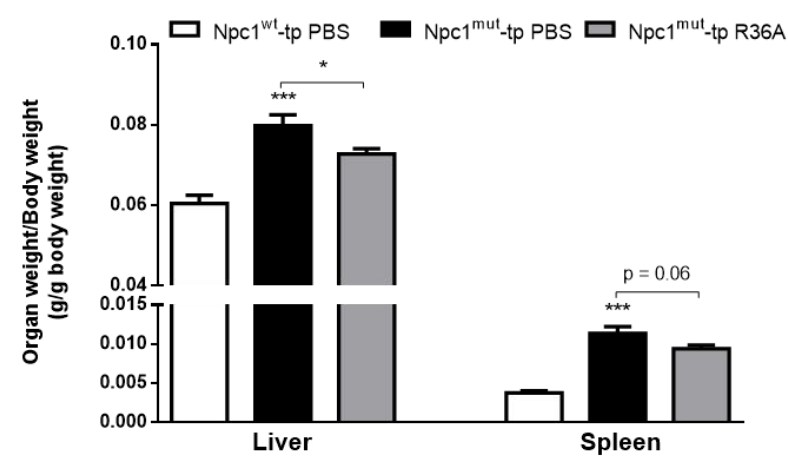

Supplemental Figure 2: Hepatosplenic pathological phenotype in Npc1mut-tp Ldlr-/- mice. (A) General histology of the liver by an HE staining of Npc1wt-tp mice and non-immunized and immunized Npc1mut-tp mice. Arrows indicate macrophage granulomas. (B) Relative liver and spleen weights. $n=9-11$ mice/group. Asterisks indicate significant difference from non-immunized Npc1wt-tp and Npc1mut-tp mice by use of two-tailed unpaired t test. $* \mathrm{p}<0.05$; *** $\mathrm{p}<0.001$. All error bars are SEM. R36A, unencapsulated Streptococcus pneumoniae. 


\section{Chapter 2}

\section{Tnf- $\alpha$}

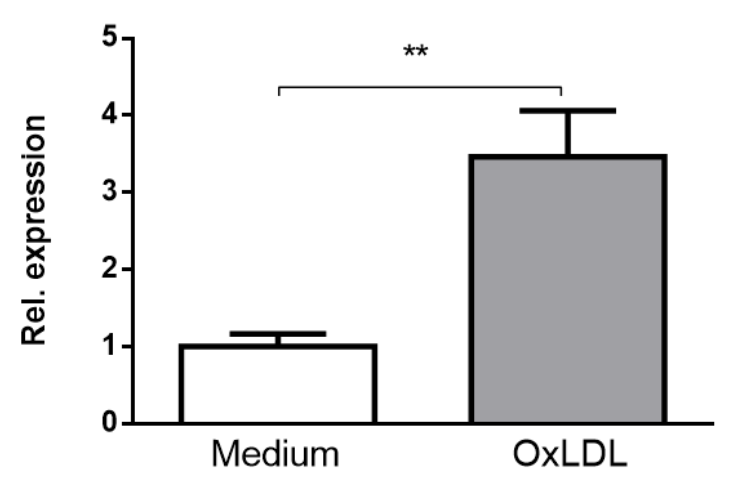

Mcp1

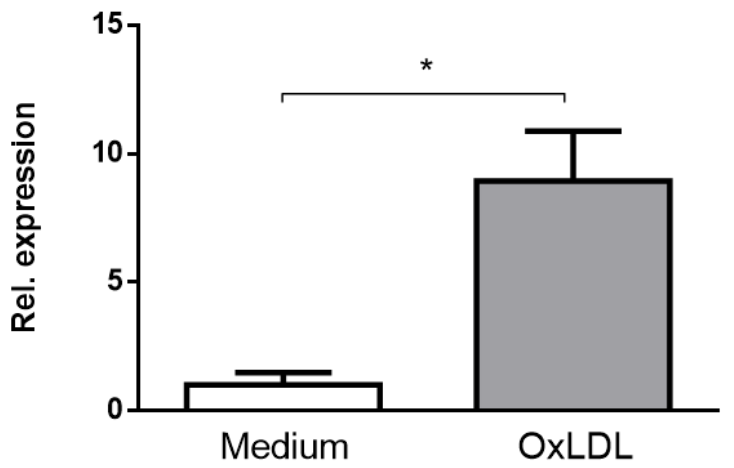

Supplemental Figure 3: Pro-inflammatory properties of oxLDL in BMDMs. Gene expression levels of the pro-inflammatory markers Tnfo and Mcp1 after oxLDL loading of wildtype BMDMs. Data are the result of three independent experiments. Asterisks indicate significant difference from control-treated BMDMs by two-tailed unpaired $\mathrm{t}$ test. $* \mathrm{p}<0.05 ; * \mathrm{p}<0.01$. All error bars are SEM.

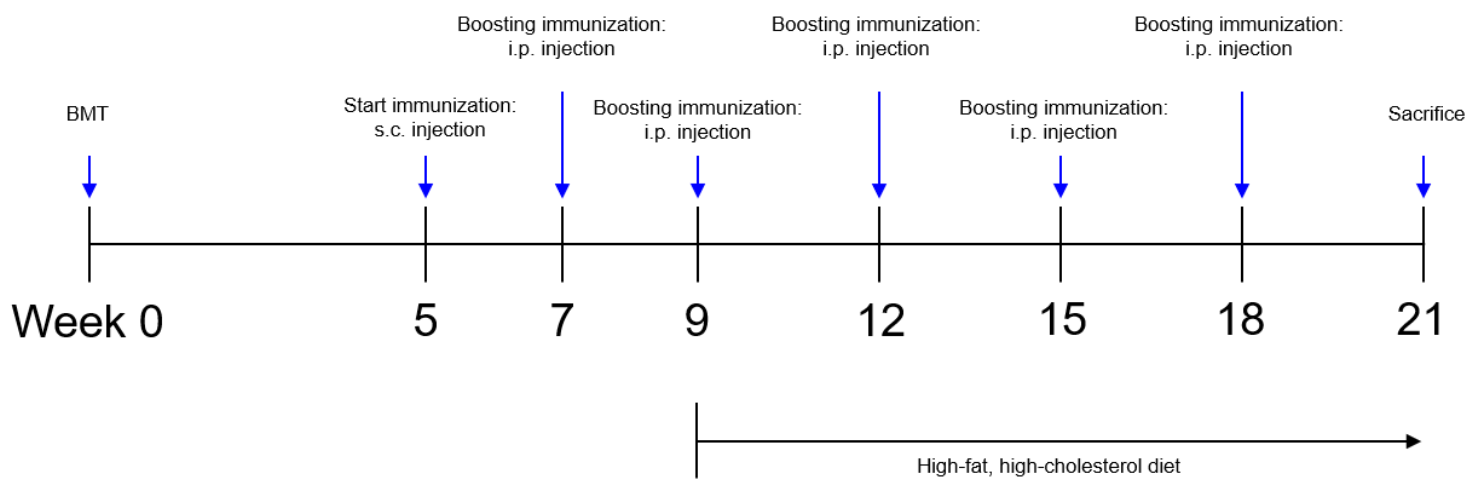

Supplemental Figure 4: Schematic overview of experimental set-up. 
Chapter 2 


\section{Chapter 3}

Cathepsin D regulates lipid metabolism in murine steatohepatitis 
Chapter 3 


\section{Chapter 3}

Chapter 3 Cathepsin D regulates lipid metabolism in murine steatohepatitis

Tom Houben, Yvonne Oligschlaeger, Tim Hendrikx, Albert V Bitorina, Sofie MA Walenbergh, Patrick J van Gorp, Marion JJ Gijbels, Silvia Friedrichs, Jogchum Plat, Frank G Schaap, Dieter Lütjohann, Marten H Hofker, Ronit Shiri-Sverdlov

*A.V.B contributed by assisting with conducting the experiments, analyzing and interpreting the data, reading and editing the manuscript.

Sci Rep. 2017 Jun 14;7(1):3494 


\title{
Chapter 3
}

\begin{abstract}
Due to the obesity epidemic, non-alcoholic steatohepatitis (NASH) is a prevalent liver disease, characterized by fat accumulation and inflammation of the liver. However, due to a lack of mechanistic insight, diagnostic and therapeutic options for NASH are poor. Recent evidence has indicated cathepsin D (CTSD), a lysosomal enzyme, as a marker for NASH. Here, we investigated the function of CTSD in NASH by using an in vivo and in vitro model. In addition to diminished hepatic inflammation, inhibition of CTSD activity dramatically improved lipid metabolism, as demonstrated by decreased plasma and liver levels of both cholesterol and triglycerides. Mechanistically, CTSD inhibition resulted in an increased conversion of cholesterol into bile acids and an elevated excretion of bile acids via the feces, indicating that CTSD influences lipid metabolism. Consistent with these findings, treating Wt BMDMs with PepA in vitro showed a similar decrease in inflammation and an analogous effect on cholesterol metabolism. Conclusion: CTSD is a key player in the development of hepatic inflammation and dyslipidemia. Therefore, aiming at the inhibition of the activity of CTSD may lead to novel treatments to combat NASH.
\end{abstract}




\section{Chapter 3}

\section{Introduction}

Due to the increasing obesity epidemic, non-alcoholic fatty liver disease (NAFLD) has emerged as the most important liver disease in the world 1. NAFLD comprises a spectrum of liver diseases ranging from benign hepatic steatosis to more advanced liver diseases such as cirrhosis and fibrosis, which eventually might result in liver failure and death. The combination of steatosis and inflammation is referred to as non-alcoholic steatohepatitis (NASH). NASH is a disease stage that can further progress into such advanced liver diseases, underscoring the value of preventing/treating the inflammatory aspect of NAFLD. Currently, the etiology and mechanisms leading to obesity-induced liver inflammation are not clear, resulting in the lack of well-defined effective therapies 2,3.

Using mice lacking the low-density lipoprotein receptor (Ldlr-/-), we previously demonstrated a positive correlation between hepatic inflammation and cholesterol accumulation in lysosomes of Kupffer cells (KCs) 4,5. Moreover, lysosomal enzymes such as cathepsin D (CTSD) are increasingly recognized for their involvement in inflammatory responses 6,7 . Whereas it was initially believed that proteases such as CTSD were only involved in non-specific protein degradation inside acidic lysosomes, research of the past decade has indicated that CTSD has a wide spectrum of functions, both of physiological and pathological nature 8,9. Under pathological inflammatory conditions, CTSD has been associated with distinct disorders such as rheumatoid arthritis, Alzheimer's disease, atherosclerosis and inflammatory bowel disease (IBD) 10-13. Furthermore, recent data also revealed a correlation between hepatic inflammation and the activity and expression of CTSD in the liver 14-16. Relevantly, plasma CTSD levels correlated with different stages of NAFLD17. These data suggest the involvement of lysosomes, and more specifically the lysosomal enzyme CTSD, in mediating the inflammatory response in NASH. However, despite the established relationship between lysosomes and inflammation as well as the awareness of an association between the lysosomal enzyme CTSD and hepatic inflammation, the exact function of CTSD in the context of NASH has not yet been investigated.

In the current study, we hypothesized that proteolytic inhibition of CTSD leads to reduced steatohepatitis. To induce NASH, Ldlr-/- mice were put on a high-fat, high-cholesterol (HFC) diet for three weeks. This model was chosen due to its close resemblance with a human-like lipoprotein profile combined with the presence of hepatic inflammation, thereby serving as a physiological model to study NASH 18. To examine whether inhibition of CTSD decreases hepatic inflammation, Ldlr-/- mice were injected with pepstatin A (PepA), an inhibitor of aspartyl proteases, for three weeks or only in the final week of the experiment. In agreement with our hypothesis, inhibition of the proteolytic activity of CTSD reduced hepatic inflammation. Remarkably, proteolytic inhibition of CTSD dramatically improved lipid and lipoprotein metabolism, which is demonstrated by decreased plasma and liver levels of both 


\section{Chapter 3}

cholesterol and triglycerides. Mechanistically, CTSD inhibition resulted in an increased conversion of cholesterol into bile acids as well as an elevated excretion of bile acids via the feces, indicating that CTSD influences lipid metabolism. Consistent with these findings, treating wildtype (Wt) bone marrow-derived macrophages (BMDMs) with PepA showed a similar decrease in inflammation and an analogous effect on cholesterol metabolism. These data demonstrate for the first time a key regulatory role for CTSD in lipid metabolism in the development of NASH. Hence, aiming at the modulation of CTSD activity in NASH holds therapeutic promise and should be further investigated in the future. 


\section{Chapter 3}

\section{Methods}

\section{Mice, diet and intervention}

Experiments were performed according to the Dutch regulation and approved by the Committee for Animal Welfare of Maastricht University. Ldlr-/- mice on a C57BL/6 background were housed under standard conditions and given free access to food and water. Female 12 week-old Ldlr-/- mice were fed either regular chow or a high-fat, high-cholesterol (HFC) diet (SAFE, Augy, France) for 3 weeks and were divided in three groups ( $\mathrm{n}=11$ for each group). The HFC diet contained $21 \%$ milk butter, $0.2 \%$ cholesterol, $46 \%$ carbohydrates and $17 \%$ casein. To examine whether proteolytic inhibition of CTSD would decrease hepatic inflammation, Ldlr-/- mice were injected intraperitoneally with pepstatin A (PepA; $50 \mu \mathrm{g} / \mathrm{g}$ body weight; P5318, Sigma, Zwijndrecht, the Netherlands), a proteolytic inhibitor of aspartyl proteases. Mice were injected with DMSO (8\%) or PepA two times every week. An overview of all experimental groups is depicted in Supplementary Fig. S1. Collection of blood and tissue specimens, fluorescence-activated cell sorting (FACS), liver histology, plasma alanine transaminase levels (ALT), RNA isolation, cDNA synthesis and qPCR were determined as described previously $4,15,41,42$.

\section{Bone marrow-derived macrophages (BMDMs)}

BMDMs were isolated from the tibiae and femurs of C57BL/6 mice. Cells were cultured in RPMI-1640 (GIBCO Invitrogen, Brede, the Netherlands) with 10\% heat-inactivated fetal calf serum (Bondinco B.V. Alkmaar, the Netherlands), penicillin $(100 \mathrm{U} / \mathrm{ml})$, streptomycin $(100 \mu \mathrm{g} / \mathrm{ml})$ and L-glutamine $2 \mathrm{mM}$ (GIBCO Invitrogen, Breda, the Netherlands), supplemented with 20\% L929-conditioned medium (LCM) for 8-9 days to generate BMDMs. After attachment, macrophages were seeded at 350,000 cells per well in 24-well plates and incubated for $24 \mathrm{~h}$ with oxLDL $(25 \mu \mathrm{g} / \mathrm{ml})$ or medium (control). Afterwards, cells were washed and exposed to PepA (10 $\mu \mathrm{g} / \mathrm{ml}$; dissolved in DMSO) or DMSO $(0.06 \%)$ for $4 \mathrm{~h}$. Next, cells were washed and stimulated with or without LPS (100 ng/ml) for 4h. Finally, conditioned medium was used for enzyme-linked immunosorbent assays and cells were lysed for mRNA expression analysis. All in vitro data are the result of at least three independent experiments.

\section{Statistical analysis}

Data were statistically analyzed by performing two-tailed unpaired t test and Area Under the Curve (AUC) analysis using GraphPad Prism version 6 for Windows. Data were expressed as the mean and standard error of the mean (SEM) and were considered significantly different compared to controltreated mice on chow $\operatorname{diet}\left(* \mathrm{p} \leq 0.05 ;{ }^{* *} \mathrm{p}<0.01 ; * * * \mathrm{p}<0.001\right)$ and compared to control-treated mice 


\section{Chapter 3}

on HFC diet (\# $\mathrm{p} \leq 0.05 ; \# \# \mathrm{p}<0.01$; \#\#\# $\mathrm{p}<0.001)$. Pearson correlation coefficients $(\mathrm{r})$ and respective $\mathrm{p}$ values were calculated to assess the statistical significance of the correlation.

Additional explanation is provided in the Supplementary Information.

\section{Results}

\section{Decreased systemic and hepatic inflammation in Ldlr-/- mice after inhibition of CTSD}

To elucidate the systemic immune effects of proteolytic inhibition of CTSD, blood leukocyte levels were determined via fluorescence-activated cell sorting after two weeks and at the end of the experiment. Administration of PepA for one week to Ldlr-/- mice on HFC diet resulted in a significant decrease of blood leukocyte, B and CD4+ T cells after 3 weeks (Fig. 1A and Supplementary Fig. S2). Though not significant, similar trends were also observed for T cells, monocytes, NK cells, Ly6Chigh monocytes, Ly6cint monocytes, Ly6Clow monocytes and CD8+ T cells, confirming the reduced systemic inflammatory phenotype after proteolytic inhibition of CTSD (Fig. 1A and Supplementary Fig. S2). In contrast, no statistical differences were observed in blood granulocyte levels between the groups (Supplementary Fig. S2). 


\section{Chapter 3}

A

Chow + control
- HFD + control
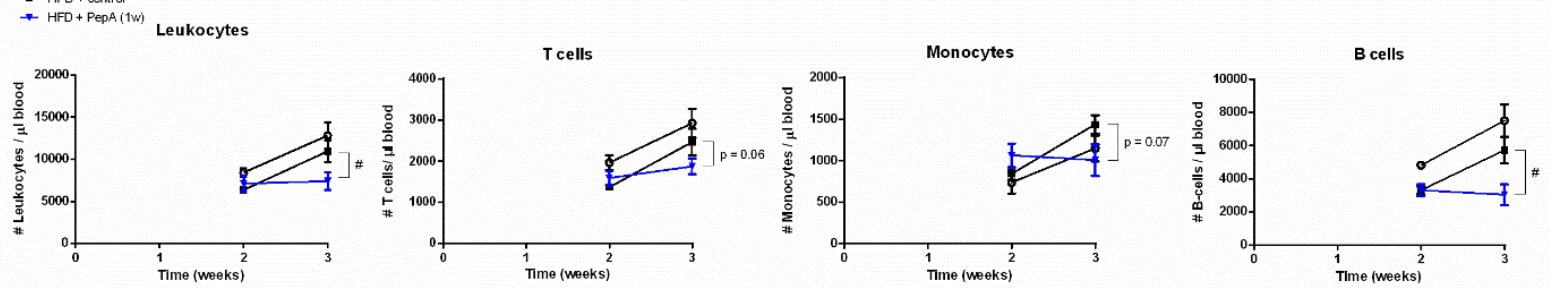

B
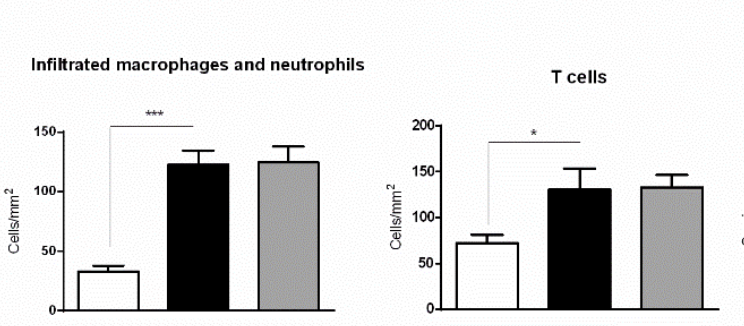

C
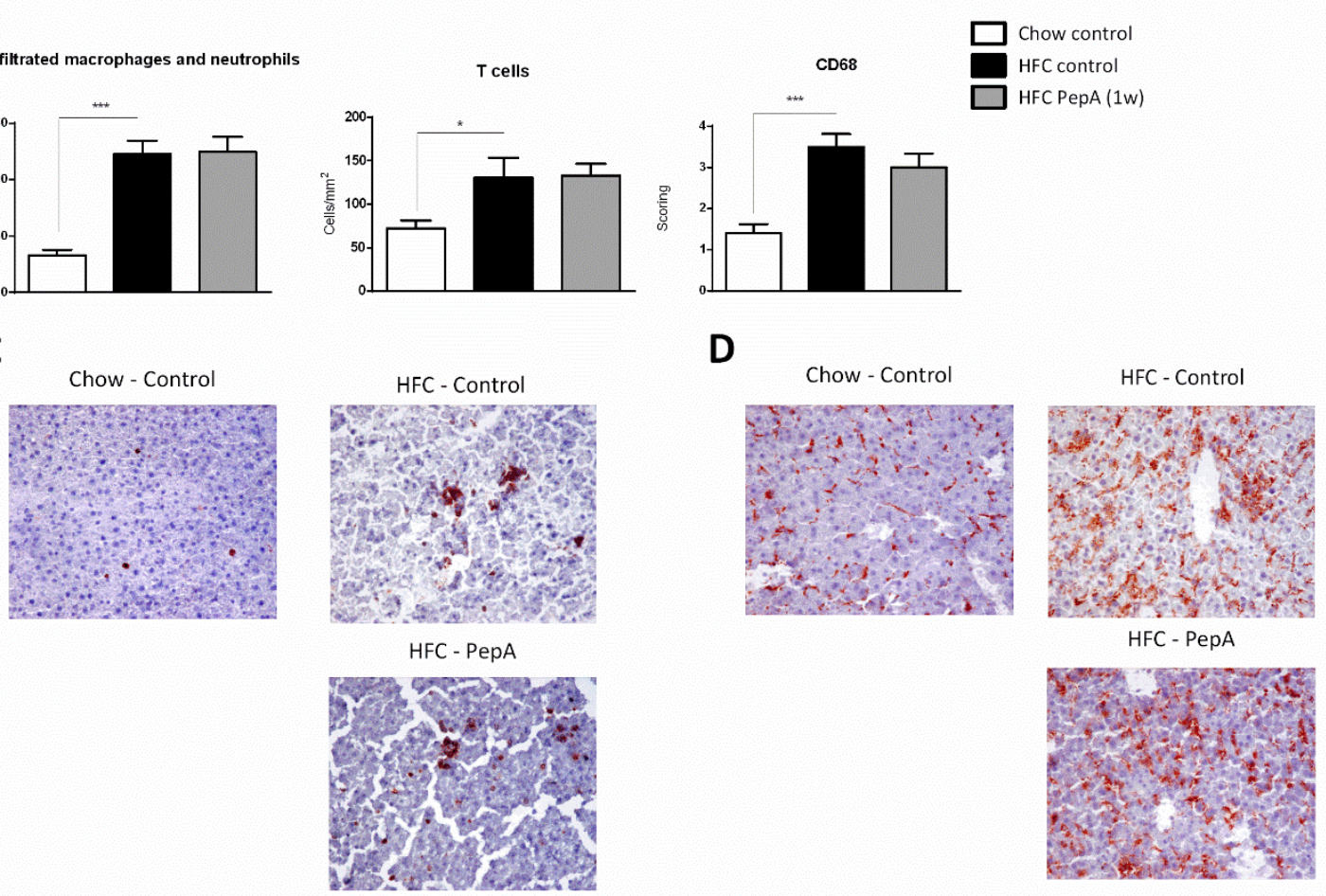

D

HFC - PepA
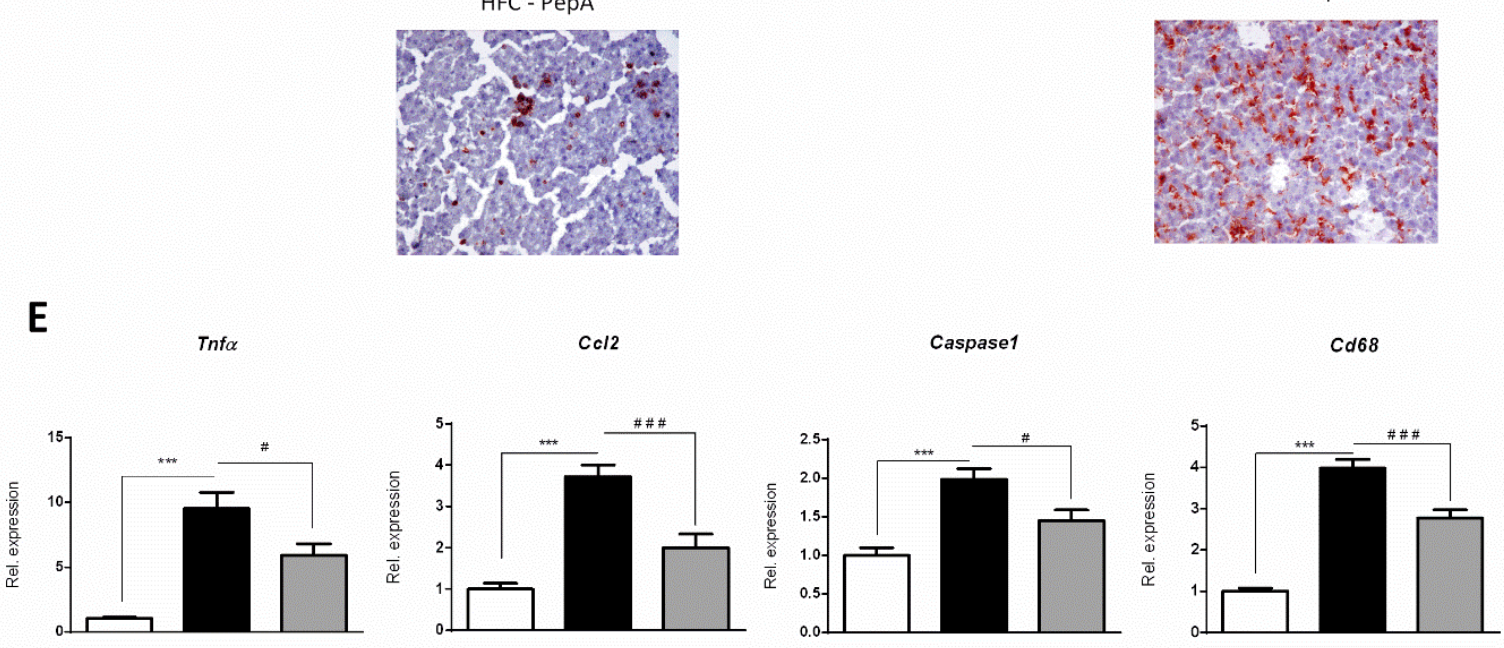

Figure 1: Parameters of systemic and hepatic inflammation in control- and PepA-injected mice. (A) Absolute levels of leukocyte, T cell, monocyte and B cell populations at week 2 and 3 of the experiment. (B) Liver sections were stained for infiltrating macrophages and neutrophils (Mac-1), T cells (CD3) and resident monocytes/macrophages (CD68). Positive cells were counted (infiltrated macrophages/neutrophils and T cells) or scored (CD68). (C,D) Representative pictures of the Mac-1 (C) and CD68 staining (D) (original magnification, 200x). (E) Gene expression analysis of Tnfa, Ccl2, Caspase1 and Cd68. Data are shown relative to control mice on chow diet. Error bars represent \pm SEM; *indicates $\mathrm{p} \leq 0.05$ and ${ }^{* * *} \mathrm{p}<0.001$ compared to mice on chow diet; \#indicates $\mathrm{p} \leq 0.05$ and \#\#\# $\mathrm{p}<0.001$ compared to control-injected mice on HFC diet by use of two-tailed unpaired $t$ test; for FACS $n=4$ animals per group and for immunohistochemistry and gene expression $n=8-11$ animals per group. 


\section{Chapter 3}

To determine the effect of the proteolytic inhibition of CTSD on hepatic inflammation, immunohistochemical stainings were performed on liver sections for the inflammatory cell markers Mac-1, CD3, CD68 and F4/80. Quantification of all immunohistochemical stainings showed no effect of one week PepA treatment on hepatic inflammation in Ldlr-/- mice on HFC diet at histological level (Fig. 1B-D and Supplementary Table S1). This observation was also confirmed by H\&E-staining (see Supplementary Fig. S3A-B). Additionally, plasma alanine transaminase levels, indicative for liver damage, did not change upon one week PepA treatment (data not shown).

As inflammatory changes at histological level usually require administration of the therapeutic agent for more than one week before these effects can be observed 19-21, we also performed hepatic gene expression analysis on the inflammatory markers tumor necrosis factor alpha (Tnf $\alpha)$, chemokine (C-XC motif) ligand-2 (Ccl2), Caspase1, cluster of differentiation 68 (Cd68), interleukin-12 (Il12) and vascular cell adhesion protein (Vcam). As shown in Fig. 1E, Supplementary Fig. S3D and Supplementary Table S1, when Ldlr-/- mice on HFC diet were treated with PepA for one week, hepatic gene expression levels of Tnfa, Ccl2, Caspase 1, Cd68 and VCAM were significantly reduced compared to control-treated Ldlr-/- mice on HFC diet (Fig. 1E). A similar trend was also observed for hepatic gene expression of IL12 and for the M2 markers cluster of differentiation 206 (Cd206), Early growth response protein 2 (Egr2) and ratio of inducible nitric oxide (iNos) and arginase 1 (Arg1) (see Supplementary Fig. 3C and Supplementary Table S1). Accordingly, hepatic protein levels of the inflammatory cytokines TNF $\alpha$ and IL12 were also reduced upon PepA administration (see Supplementary Table S1), indicating CTSD as a contributing compound in evoking the hepatic inflammatory response. Moreover, when injected over the total three weeks, Ldlr-/- mice showed a nearly significant decrease of hepatic $\mathrm{T}$ cell levels, which was combined with an even stronger reduction of hepatic inflammatory markers at gene and protein expression level (see Supplementary Table S1). Altogether, these data demonstrate that inhibition of the proteolytic site of CTSD in Ldlr-/- mice reduces the development of systemic inflammation and hepatic inflammatory gene expression, thereby pointing to a pro-inflammatory role of CTSD. 


\section{Chapter 3}

\section{Improved hepatic lysosomal function after inhibition of intracellular and circulating CTSD is linked to hepatic inflammation in Ldlr-/- mice}

To investigate the effect of PepA on hepatic lysosomal function in diet-induced hepatic inflammation, plasma and hepatic CTSD activity as well as the activity the lysosomal enzyme acid phosphatase (AP) were measured in total liver. Treating Ldlr-/- mice on HFC diet with PepA for one week resulted in a striking decrease of plasma CTSD activity compared to control-treated mice (Fig. 2A). Furthermore, PepA-treated Ldlr-/- mice on HFC diet demonstrated a reduction in hepatic CTSD activity (Fig. 2B). In contrast, one week PepA-treatment did neither affect plasma nor hepatic cathepsin E (CTSE) activity (Supplementary Fig. 4A-B). Collectively, these results indicate that PepA is a proteolytic inhibitor of intracellular and circulating CTSD. Of note, while Ldlr-/- mice on a HFC diet showed increased plasma and hepatic CTSD activity compared to Ldlr-/- mice on chow diet (Fig. 2A-B), when compared to Wt mice on HFC diet, plasma CTSD (and not hepatic CTSD) activity increased in HFC-fed Ldlr-/- mice (data not shown). These data suggest that circulating CTSD likely plays the most important role in developing hepatic inflammation.

A

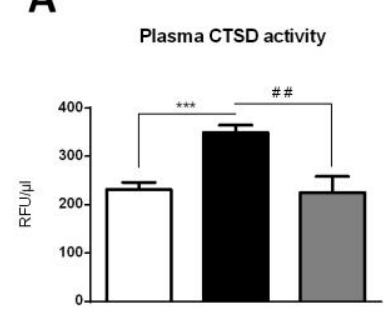

D

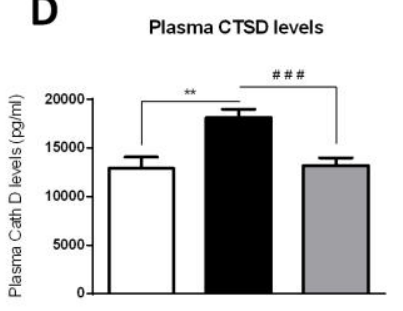

B

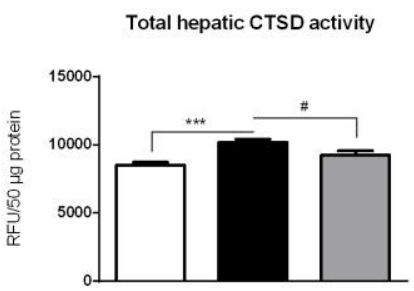

C

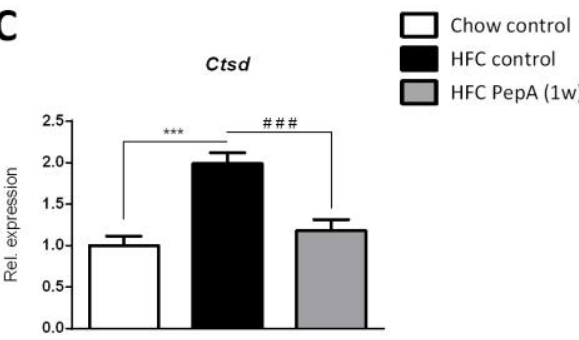

$\mathbf{F}$

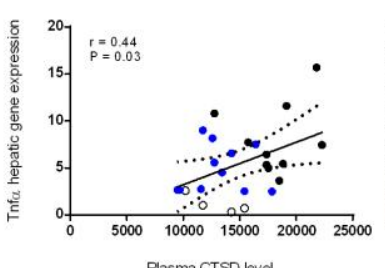

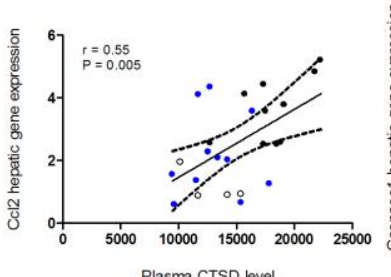

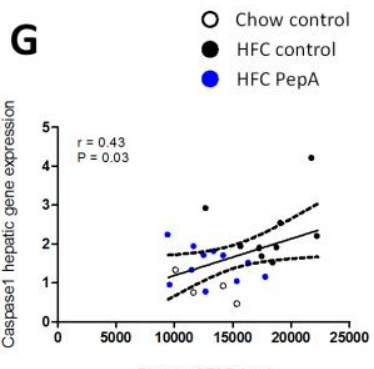

Plasma CTSD level

Figure 2: Parameters of lysosomal function in control- and PepA-injected mice. (A,B) Plasma (A) and hepatic (B) activity of CTSD. (C) Hepatic gene expression analysis of Ctsd. Data are shown relative to control mice on chow diet by use of two-tailed unpaired t test. (D) Plasma levels of CTSD. (E-G) Correlation between plasma levels of CTSD and hepatic gene expression of Tnfa, Ccl2 and Caspase1 by Pearson correlation. Error bars represent \pm SEM. ${ }^{*}$ Indicates $p<0.01$ and ${ }^{* * *} \mathrm{p}<0.001$ compared to mice on chow diet; \#indicates $\mathrm{p} \leq 0.05$, \#\# $\mathrm{p}<0.01$ and \#\# $\mathrm{p}<0.001$ compared to control-injected mice on HFC diet by use of two-tailed unpaired t test. $\mathrm{n}=9-11$ animals in each group. For correlation analysis, $\mathrm{n}=4$ animals on chow diet and $\mathrm{n}=10$ for mice on HFC diet. 


\section{Chapter 3}

Subsequently, the activity of acid phosphatase (AP) was measured in the liver. Although total hepatic AP activity was similar among all groups, control-treated mice on a HFC diet for 3 weeks showed reduced levels of lysosomal AP activity compared to mice on chow diet, confirming lysosomal dysfunction upon HFC diet (see Supplementary Fig. S4C-D). In contrast, PepA-treated Ldlr-/- mice on HFC diet demonstrated elevated levels of hepatic lysosomal AP activity compared to control mice on HFC diet (see Supplementary Fig. S4D). To confirm these effects on lysosomal function, hepatic gene expression was measured for the lysosomal enzymes cathepsin D (Ctsd), cathepsin S (Ctss) and Ap. As shown in Fig. 2C and Supplementary Fig. S4E, hepatic gene expression of Ctsd, Ctss and Ap returned to chow levels after inhibition of CTSD, confirming the rebalancing of lysosomal function after CTSD inhibition. Additionally, to validate whether plasma CTSD levels correlate with the level of hepatic inflammation, plasma CTSD levels were measured. Ldlr-/- mice on an HFC diet showed increased levels of plasma CTSD levels, whereas mice that received the PepA treatment, showed reduced plasma CTSD levels (Fig. 2D). Moreover, hepatic gene expression levels of the inflammatory markers Tnfa, Ccl2 and Caspase1 correlated significantly with plasma levels of CTSD (Fig. 2E), underlining the link between hepatic inflammation and plasma CTSD levels. Together, these data reveal that proteolytic inhibition of intracellular and circulating CTSD improves diet-induced hepatic lysosomal dysfunction, which is connected to hepatic inflammation in Ldlr-/- mice.

\section{Improved lipid and lipoprotein metabolism in PepA-treated Ldlr-/- mice on HFC diet}

To determine whether inhibition of CTSD affects lipid metabolism, plasma and hepatic lipid levels were measured. Whereas plasma cholesterol and triglycerides were elevated upon feeding an HFC diet, administration of PepA significantly decreased both cholesterol and triglycerides in the plasma (Fig. 3A-B). As shown in the fast protein liquid chromatography (FPLC) profiles, the reductions of both lipids were mainly attributed to a decrease in the VLDL fraction (see Supplementary Fig. S5A-D). Similar to plasma cholesterol and triglyceride levels, hepatic cholesterol and triglyceride levels were also elevated in control-treated Ldlr-/- mice on a HFC diet and decreased upon treatment with PepA (Fig. 3C-D). These changes in hepatic lipid levels were confirmed by Oil red O staining (Fig. 3E and Supplementary Fig. S5E) and are in line with the changes observed in the relative liver weights of these mice (Supplementary Fig. S5F). 


\section{Chapter 3}

A

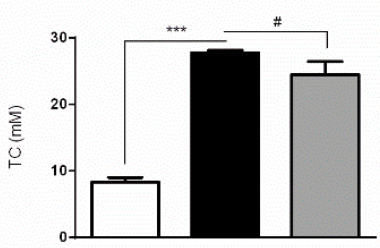

C

Hepatic total cholesterol

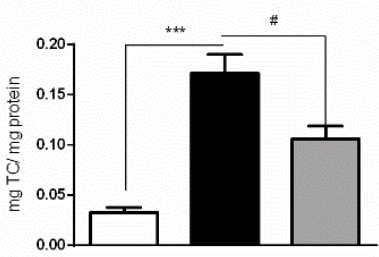

$\mathbf{E}$

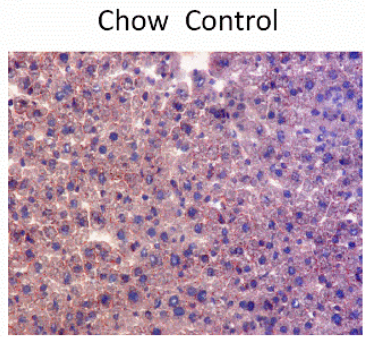

B Plasma triglycerides

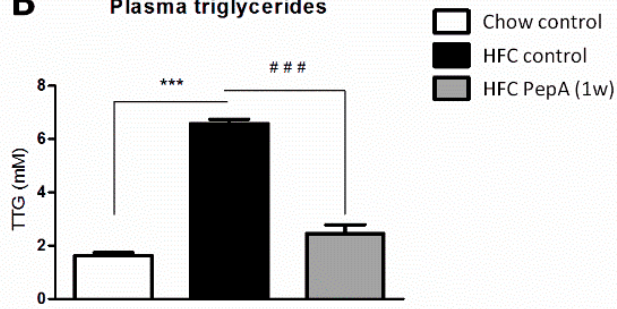

D Hepatic total triglycerides

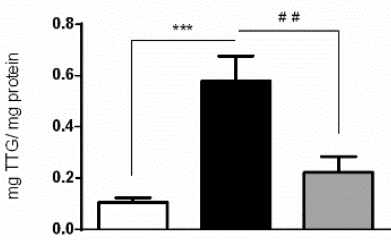

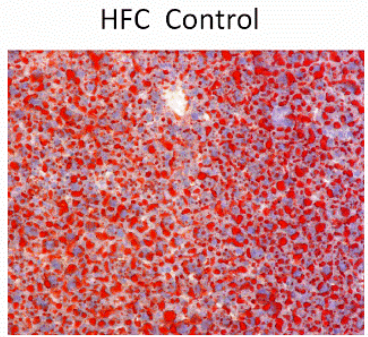

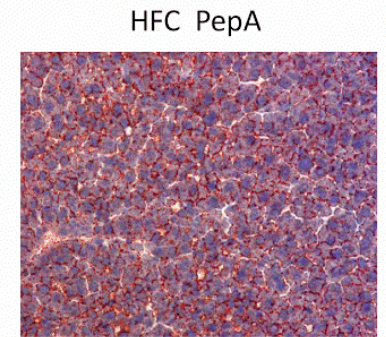

F

$c d 36$

Npc2

Lxro

Ppar- $\gamma$
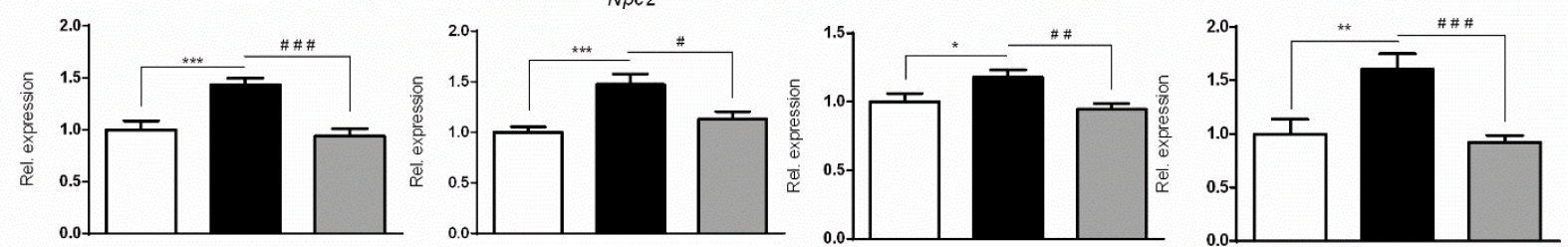

Figure 3: Plasma and hepatic lipids in hyperlipidemic mice with or without PepA-treatment. (A,B) Plasma total cholesterol and triglyceride measurements. (C,D) Total hepatic cholesterol and hepatic triglyceride measurements. (E) Representative pictures (original magnification, 200x) of the Oil red O staining. (F) Gene expression analysis of Cd36, Npc2, Lxra and Ppar- $\gamma$. Gene expression data are shown relative to control mice on chow diet. Error bars represent $\pm \mathrm{SEM}$; *indicates $\mathrm{p} \leq 0.05,{ }^{* *} \mathrm{p}<0.01$ and ${ }^{* *} \mathrm{p}<0.001$ compared to mice on chow diet; \#indicates $\mathrm{p} \leq 0.05$, \#\# $\mathrm{p}<0.01$ and \#\# $\mathrm{p}<0.001$ compared to control-injected mice on HFC diet by use of two-tailed unpaired $\mathrm{t}$ test. $\mathrm{n}=9$ 11 animals for each group analysis.

To further define the influences of PepA-mediated CTSD inhibition on hepatic lipid metabolism, hepatic gene expression analysis was performed on markers involved in lipid homeostasis. Compared to control-injected Ldlr-/- mice on HFC diet, PepA administration resulted in reduced gene expression levels of Cluster of differentiation 36 (Cd36), Niemann-Pick C2 (Npc2) and Liver X receptor alpha (Lxr $\alpha$ ), suggesting a reduced uptake, intralysosomal presence and efflux of cholesterol in these mice 


\section{Chapter 3}

respectively (Fig. 3F). In addition, peroxisome proliferator-activated receptor gamma (Ppar- $\gamma$ ) expression levels, a lipid-activated transcription factor of genes controlling lipid metabolism, were also decreased upon PepA administration, indicating a dramatic improvement in lipid homeostasis (Fig. 3F).

As the proteolytic inhibition of CTSD also appeared to influence hepatic cholesterol metabolism, hepatic synthesis and degradation of cholesterol were investigated in more detail. Inhibition of CTSD by PepA decreased hepatic cholesterol synthesis, as shown by measurements of hepatic desmosterol levels (Fig. 4A). This finding was confirmed by gene expression levels of the rate-limiting enzyme for cholesterol synthesis, 3-hydroxy-3-methyl-glutaryl-Coenzyme A reductase (HmG-CoAR) (Fig. 4B). Besides cholesterol synthesis, also cholesterol degradation was affected upon inhibition of CTSD. Specifically, whereas also 27-hydroxcholesterol (27HC; see Supplementary Fig. S6A) levels were nearly significantly elevated, the conversion of cholesterol into 7 alpha-hydroxycholesterol $(7 \alpha \mathrm{HC})$ was significantly increased in PepA-injected Ldlr-/- mice on a HFC diet compared to control-injected mice on HFD diet (Fig. 4C). In line, hepatic gene expression levels of the cytochrome P450 7A1 (Cyp7a1) enzyme, responsible for this conversion, were elevated in PepA-treated Ldlr-/- mice on a HFC diet, corroborating increased cholesterol degradation after inhibition of CTSD (Fig. 4D). Furthermore, whereas plasma and hepatic bile acid levels remained unaffected (see Supplementary Fig. S6B and S6C), PepA-treatment increased fecal bile acid (significant) (Fig. 4E) and cholesterol (not significant) levels (Fig. 4F). Overall, these data indicate that inhibition of CTSD activity results in increased bile acid synthesis eventually leading to excretion via the feces, thereby indicating an effect of CTSD on lipid metabolism. 


\section{Chapter 3}

A

Hepatic desmosterol

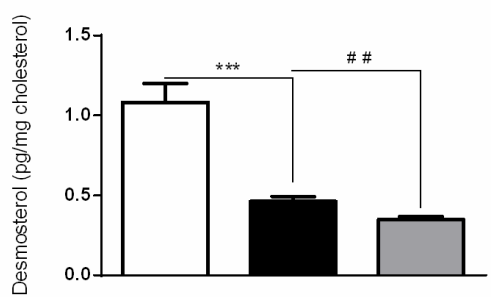

C

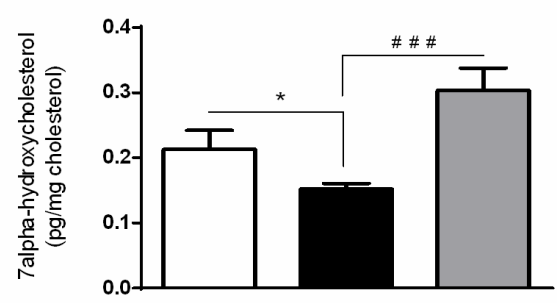

E

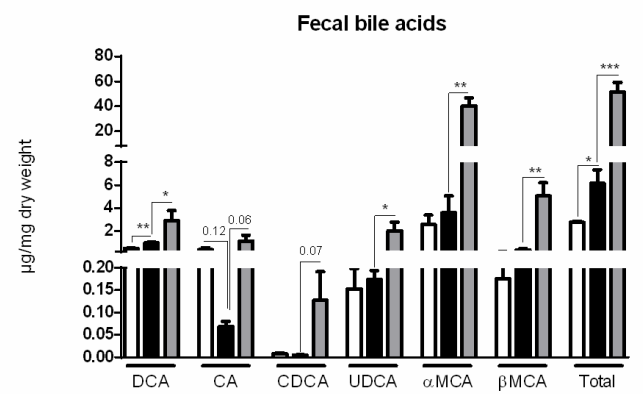

B

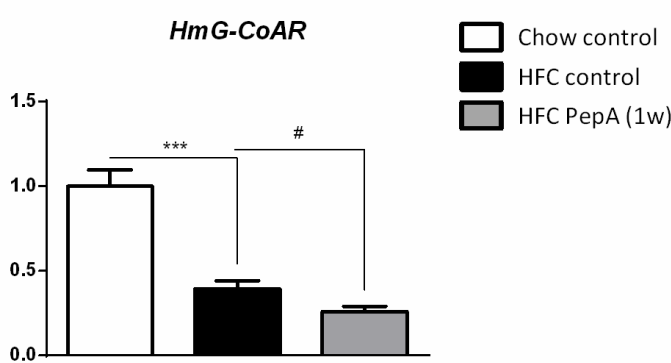

D

Cyp7a1

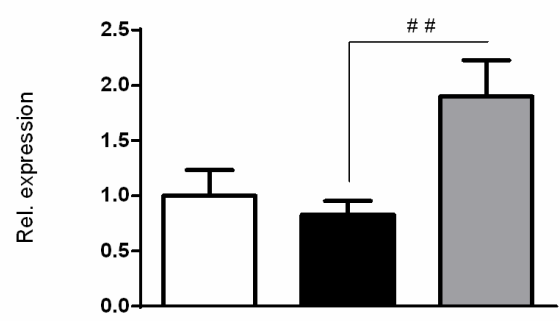

$\mathbf{F}$

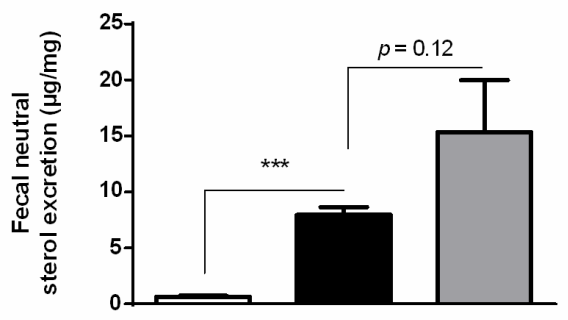

Figure 4: Hepatic de novo synthesis and degradation of cholesterol. (A,C) Hepatic concentrations of desmosterol (A) and 7 alphahydroxycholesterol $(7 \alpha \mathrm{HC} ; \mathrm{C})$. Both values are shown relative to the hepatic cholesterol concentrations. (B,D) Gene expression analysis of HmG-CoAR and Cyp7a1. (E,F) Fecal bile acid (E) and cholesterol levels (F). Both measurements are relative to mg dry weight of the fecal sample. Gene expression data are shown relative to control mice on chow diet. Error bars represent \pm SEM; *indicates $\mathrm{p} \leq 0.05$ and $* * * p<0.001$ compared to mice on chow diet; \#indicates $\mathrm{p} \leq 0.05$, \#\# $\mathrm{p}<0.01$ and \#\#\# $\mathrm{p}<0.001$ compared to control-injected mice on HFC diet by use of two-tailed unpaired $\mathrm{t}$ test; $\mathrm{n}=11$ for each group. DCA, deoxycholic acid; CA, cholic acid; CDCA, chenodeoxycholic acid; UDCA, ursodeoxycholic acid; $\alpha \mathrm{MCA}, \alpha$-muricholic acid; $\beta$ MCA, $\beta$-muricholic acid.

\section{Inhibition of the proteolytic function of CTSD reduces inflammation in oxLDL-loaded BMDMs}

In order to explore the specific role of macrophages on the reduced inflammatory response upon CTSD inhibition, bone marrow cells of Wt mice were isolated, differentiated to macrophages and incubated with oxLDL for $24 \mathrm{hr}$. Subsequently, cells were treated with PepA for $4 \mathrm{hr}$, followed by $4 \mathrm{hr}$ stimulation with lipopolysaccharide (LPS). Upon incubation with PepA, cytokine levels of the pro-inflammatory marker $\mathrm{TNF} \alpha$, measured in the supernatant of the BMDMs, were significantly reduced compared to 


\section{Chapter 3}

carrier (dimethyl sulfoxide (DMSO))-treated cells (Fig. 5A, left panel). Additionally, cytokine levels of the anti-inflammatory marker IL10 increased after PepA treatment (Fig. 5A, right panel), confirming the reduction in inflammation upon PepA treatment and the pro-inflammatory properties of CTSD in lipid-induced inflammation (as CTSE activity was also here not affected by PepA (see Supplementary Fig. S7). In line, Tnfa gene expression measured in BMDMs showed a decrease after PepA treatment. A similar trend was also observed for the expression of $\mathrm{Ccl} 2$, but this did not reach statistical significance (Fig. 5B, right panel). The aggregated in vitro findings are in line with a reduction in hepatic inflammation upon PepA treatment, and by extension a pro-inflammatory action of CTSD.

\section{A}
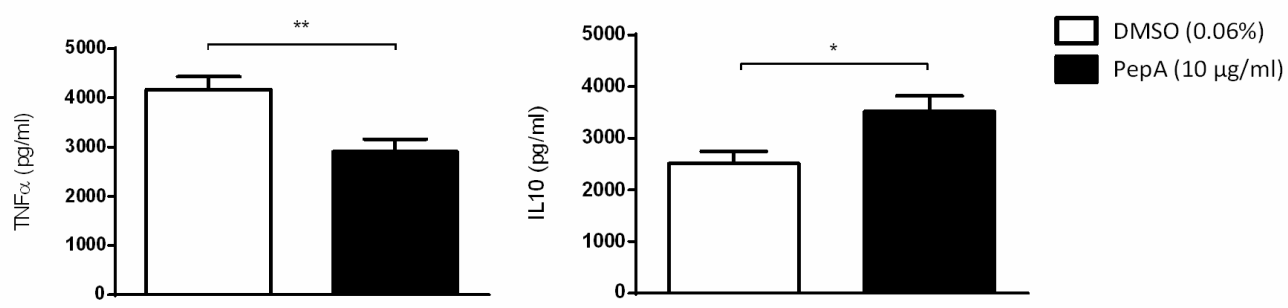

B

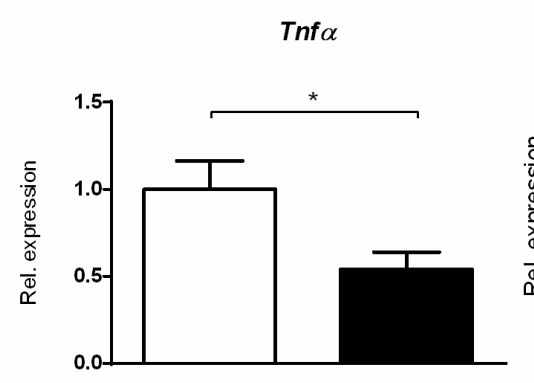

C
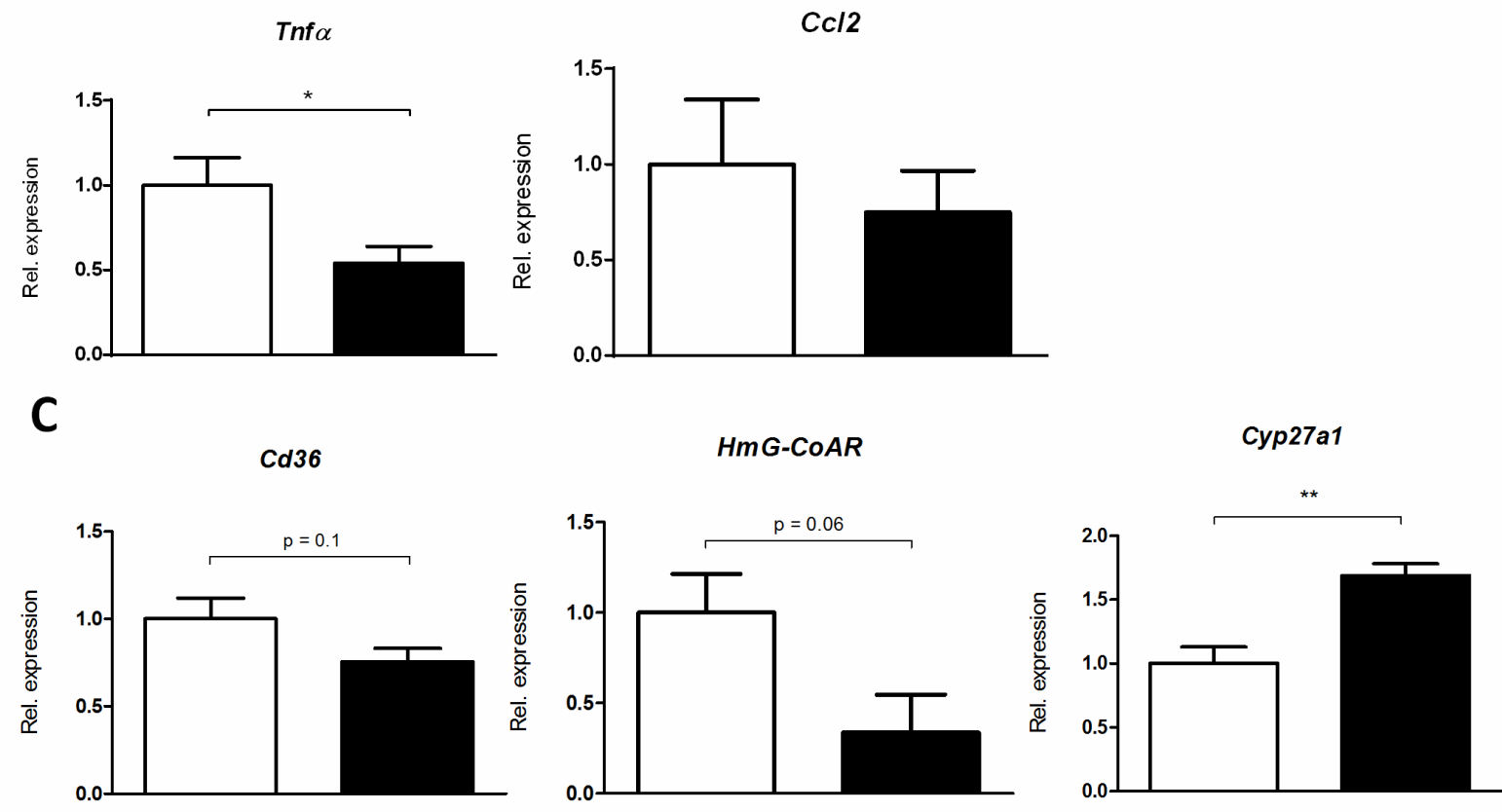

Figure 5: Gene expression and cytokine levels of oxLDL-loaded bone marrow-derived macrophages (BMDM) treated with PepA. (A) TNF $\alpha$ and IL10 cytokine secretion in supernatant of Wt mouse BMDMs, exposed to oxLDL for $24 \mathrm{hr}$ and, subsequently, treated with or without PepA (4 hr) and LPS (4 hr). (B,C) Gene expression analysis of Tnfa, Ccl2, Cd36, HmG-CoAR and Cyp27a1 measured in Wt mouse BMDMs. Gene expression data are shown relative to DMSO-treated BMDMs. All data represent the mean value of three or four independent experiments (with $\mathrm{n}=13-16$ per group). Error bars represent \pm SEM; $*$ indicates $\mathrm{p} \leq 0.05,{ }^{* *} \mathrm{p}<0.01$ and $* * * \mathrm{p}<0.001$ compared to DMSOtreated bone marrow-derived macrophages by use of two-tailed unpaired $t$ test. 


\section{Chapter 3}

To study whether the PepA-dependent reduction in inflammation was accompanied by an improvement in cholesterol metabolism, gene expression analysis was performed for Cd36, Cyp27a1 and HmGCoAR. In line with the hepatic in vivo gene expression data, cholesterol uptake $(\mathrm{Cd} 36)$ and synthesis (HmG-CoAR) were reduced, though not significant, after PepA treatment in BMDMs. Next, Cyp27a1, the main enzyme responsible for the degradation of cholesterol in macrophages, appeared to be upregulated, indicating higher levels of cholesterol degradation in BMDMs (Fig. 5C). Thus, oxLDLloaded BMDMs demonstrate a reduced inflammatory response upon proteolytic inhibition of CTSD, likely caused by an improved cholesterol metabolism.

\section{Discussion}

A major impediment to the development of well-defined, effective therapies for NASH has been the lack of understanding the mechanisms leading to the inflammatory component of this disorder. In the current study, we show for the first time that the lysosomal enzyme CTSD is a key player in the pathogenesis of NASH. Mechanistically, our data suggest that the pro-inflammatory function of CTSD is tied to a regulation of general lipid metabolism. In addition, we provide evidence for the central role of macrophages in exerting the lipid-induced effects of CTSD in the liver. These findings unravel CTSD as a promising, novel target for the treatment of NASH.

While CTSD was shown to have anti-inflammatory properties in the context of neutrophils 22, ample evidence points towards the pro-inflammatory effects of CTSD. In human intestinal epithelial cells and a mouse model for IBD, it was shown that activation of the lysosomal enzyme CTSD contributes to intestinal inflammation 12,23,24. Furthermore, inhibition of CTSD and CTSS reduced cardiovascular inflammation and attenuated atherosclerotic lesion progression, reiterating the contribution of cathepsins to pathological inflammatory responses 7,11,25,26. Notably, in the aforementioned IBD and atherosclerotic studies, the pro-inflammatory effects of CTSD were suggested to be derived from macrophages 7,12,23,24. Indeed, lysosomal enzymes in macrophages appear to play a critical role in withstanding the lipid challenge in obesity-induced inflammatory processes 27,28 . In line, cytokine release was reduced in oxLDL-loaded BMDMs treated with PepA, substantiating the contribution of macrophages to CTSD-mediated hepatic inflammation. Surprisingly, hepatic macrophage levels (indicated by CD68 quantification) were not reduced after CTSD inhibition. Potential explanations for this finding might be a delayed egress of macrophages from the liver upon resolution of inflammation 21 or due to a prolonged half-life of macrophages 20,29. Relevantly, while we demonstrate a positive correlation between hepatic CTSD expression and hepatic inflammation, Fukuo et al. showed reduced 


\section{Chapter 3}

levels of hepatic CTSD in NAFLD patients 14. However, these patients were not evaluated for the presence of inflammation and, thereby, cannot be used to link hepatic inflammation to reduced CTSD expression. Together, our current observations are in line with a causal relation between lipid-induced lysosomal dysfunction in hepatic macrophages and the level of inflammation in the liver 15 . Thus, our data point towards a pivotal role for macrophage-derived CTSD in the development of hepatic inflammation.

Relevantly, proteolytic inhibition of CTSD resulted in reduced cholesterol and triglyceride levels in plasma and liver. This finding suggests that the improved NASH-phenotype in PepA-treated Ldlr-/mice is due to an effect of CTSD on lipid metabolism. In agreement with this finding, prevention of the proteolytic activation of the lysosomal enzyme acid sphingomyelinase was shown to reduce cholesterol and triglyceride levels in the liver 30. Therefore, the influence of CTSD on lipid metabolism, as observed in this study, might not be unique to CTSD, but could rather be a shared mechanism of several lysosomal enzymes to control lipid homeostasis. In support of this concept, a deficiency in the gene encoding for CTSD results in a specific type of Batten disease, a disorder which is characterized by the cellular accumulation of lipid-containing residues 31,32.

There is considerable evidence contributing to the concept that a disturbance in bile acid metabolism interferes with cholesterol and, by extension, triglyceride metabolism. For example, a deficiency in the enzyme CYP7A1 was shown to lead to a hypercholesterolemic phenotype 33, which is in line with our results that showed the same inverse relation between Cyp7a1 and plasma cholesterol levels. Of note, patients with CYP7A1 deficiency were also reported to be hypertriglyceridemic. In agreement, triglyceride homeostasis and bile acid metabolism were revealed to be closely intertwined by regulation of farnesoid X receptor (FXR) and small heterodimer partner (SHP) on sterol regulatory elementbinding protein (SREBP-1c), which is a known transcription factor of fatty acid and cholesterol synthesis 34,35 . Similar to cholesterol levels, plasma and liver triglyceride levels in our study were inversely correlated with $7 \alpha \mathrm{HC}$ levels, the first precursor of the classical pathway of bile acid formation. In addition, hypertriglyceridemia has been associated with increased systemic inflammation 36. Therefore, the overall observed effects on lipid metabolism in our study might be explained by a direct link between CTSD and bile acid metabolism. In agreement with this concept, though bile acids levels in plasma and liver were similar, fecal levels were dramatically increased after CTSD inhibition. Therefore, our data indicate that modulation of CTSD has the potential to increase bile acid excretion, resulting in reduced lipid levels in hyperlipidemic conditions (Fig. 6). 


\section{Chapter 3}

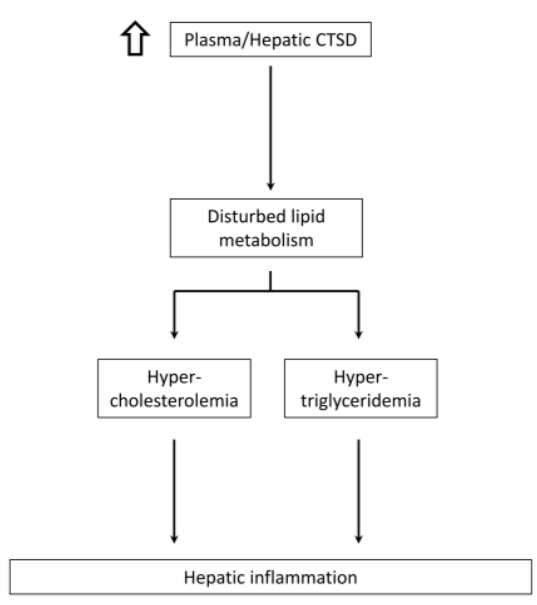

Figure 6: Schematic overview illustrating the proposed by which CTSD participates in the development of hepatic inflammation. Based on findings of the current study, increased levels of CTSD lead to a disturbance in lipid metabolism, which is known to result in increased plasma cholesterol (hypercholesterolemia) and triglyceride (hypertriglyceridemia) levels. Consequently, hypercholesterolemia and hypertriglyceridemia can lead to hepatic inflammation.

The observation that both plasma (circulating) and hepatic (intracellular) CTSD activity were decreased after PepA inhibition suggests that the results in this study are derived from intra- and extracellular effects of CTSD. Indeed, intracellular CTSD-induced apoptosis was demonstrated to induce inflammation in the liver 37. Therefore, though the strongest decrease in activity was observed in the circulating fraction of CTSD, the observed effects are likely a combination of the modulation of intra- and extracellular CTSD. Furthermore, besides CTSD, PepA can also inhibit the aspartyl protease CTSE, renin and pepsin, arguing the specificity for CTSD. Renin- and pepsin are proteases found in the kidney (renin) and digestive system (pepsin). As the reductions in inflammation were also observed in vitro, it is unlikely that either of them are involved in the observed effects in this study. Additionally, CTSE activity was unaffected in plasma, liver and BMDMs after PepA treatment, suggesting that the observed effects are mainly related to the inhibition of CTSD. Yet, it cannot be completed excluded that, PepA also influenced other processes that contributed to the observed inflammatory effects in this study.

Enhancing lysosomal exocytosis in several lysosomal storage diseases strongly reduced substrate accumulation, suggesting a pivotal role for this mechanism in the setting of cellular homeostasis 38 . In the context of lipid storage, exocytosis of lysosomes may be a compensatory mechanism to 'dump' undigested lipids into the extracellular space as well as an elegant way to conduct signaling to protect the cell from lipid overload. In line with this concept, elevated plasma CTSD levels in mice on HFC diet were diminished after reduction of liver lipid levels. As such, the excretion of lysosomal enzymes may be a secondary consequence of a survival mechanism to protect the cell from lipid overload. Furthermore, changes in lysosomal pH have been shown to alter processing and secretion of CTSD 39. Notably, intracellular accumulation of poorly degradable, oxidized lipid-protein cross-links was shown to alter the turnover of CTSD, leading to mistargeting of CTSD into the extracellular space 40. These data suggest that, by targeting specifically the circulating fraction of CTSD, lipid levels may be adjusted. 


\section{Chapter 3}

In summary, our study elucidates a key role for CTSD in the development of NASH. Our data suggest a novel mechanism by which CTSD affects lipid metabolism. Future studies are necessary to determine the exact underlying mechanisms explaining the observed effect in this study and whether other lysosomal enzymes possess similar effects as CTSD. Taken together, targeting CTSD has the potential to become an auspicious, therapeutic strategy for NASH.

\section{References}

1 Loomba, R. \& Sanyal, A. J. The global NAFLD epidemic. Nat Rev Gastroenterol Hepatol 10, 686-690, doi:10.1038/nrgastro.2013.171 (2013).

2 Ray, K. NAFLD-the next global epidemic. Nat Rev Gastroenterol Hepatol 10, 621, doi:10.1038/nrgastro.2013.197 (2013).

3 Nascimbeni, F. et al. From NAFLD in clinical practice to answers from guidelines. J Hepatol 59, 859-871, doi:10.1016/j.jhep.2013.05.044 (2013).

4 Bieghs, V. et al. Internalization of modified lipids by CD36 and SR-A leads to hepatic inflammation and lysosomal cholesterol storage in Kupffer cells. PLoS One 7, e34378, doi:10.1371/journal.pone.0034378 (2012).

5 Bieghs, V. et al. Specific immunization strategies against oxidized low-density lipoprotein: a novel way to reduce nonalcoholic steatohepatitis in mice. Hepatology 56, 894-903, doi:10.1002/hep.25660 (2012).

6 Bordon, Y. Immune regulation: lysosomes at the heart of inflammation. Nat Rev Immunol 11, 502, doi:10.1038/nri3035 (2011).

7 Sukhova, G. K. et al. Deficiency of cathepsin S reduces atherosclerosis in LDL receptordeficient mice. J Clin Invest 111, 897-906, doi:10.1172/JCI14915 (2003).

8 Benes, P., Vetvicka, V. \& Fusek, M. Cathepsin D--many functions of one aspartic protease. Crit Rev Oncol Hematol 68, 12-28, doi:10.1016/j.critrevonc.2008.02.008 (2008).

9 Khalkhali-Ellis, Z. \& Hendrix, M. J. Two Faces of Cathepsin D: Physiological Guardian Angel and Pathological Demon. Biology and medicine 6, doi:10.4172/0974-8369.1000206 (2014).

10 Cataldo, A. M. \& Nixon, R. A. Enzymatically active lysosomal proteases are associated with amyloid deposits in Alzheimer brain. Proc Natl Acad Sci U S A 87, 3861-3865 (1990).

11 Hakala, J. K. et al. Lysosomal enzymes are released from cultured human macrophages, hydrolyze LDL in vitro, and are present extracellularly in human atherosclerotic lesions. Arterioscler Thromb Vasc Biol 23, 1430-1436, doi:10.1161/01.ATV.0000077207.49221.06 (2003).

12 Hausmann, M. et al. Cathepsin D is up-regulated in inflammatory bowel disease macrophages. Clinical and experimental immunology 136, 157-167, doi:10.1111/j.1365-2249.2004.02420.x (2004). 


\section{Chapter 3}

13 Poole, A. R. et al. Secretion and localization of cathepsin D in synovial tissues removed from rheumatoid and traumatized joints. An immunohistochemical study. Arthritis and rheumatism 19, 1295-1307 (1976).

14 Fukuo, Y. et al. Abnormality of autophagic function and cathepsin expression in the liver from patients with non-alcoholic fatty liver disease. Hepatology research : the official journal of the Japan Society of Hepatology 44, 1026-1036, doi:10.1111/hepr.12282 (2014).

Bieghs, V. et al. The cholesterol derivative 27-hydroxycholesterol reduces steatohepatitis in mice. Gastroenterology 144, 167-178 e161, doi:10.1053/j.gastro.2012.09.062 (2013).

16 Hendrikx, T. et al. Macrophage specific caspase-1/11 deficiency protects against cholesterol crystallization and hepatic inflammation in hyperlipidemic mice. PLoS One 8, e78792, doi:10.1371/journal.pone.0078792 (2013).

17 Walenbergh, S. M. et al. Plasma cathepsin D correlates with histological classifications of fatty liver disease in adults and responds to intervention. Sci Rep 6, 38278, doi:10.1038/srep38278 (2016).

18 Bieghs, V. et al. LDL receptor knock-out mice are a physiological model particularly vulnerable to study the onset of inflammation in non-alcoholic fatty liver disease. PLoS One 7, e30668, doi:10.1371/journal.pone.0030668 (2012).

19 Vogel, C. \& Marcotte, E. M. Insights into the regulation of protein abundance from proteomic and transcriptomic analyses. Nat Rev Genet 13, 227-232, doi:10.1038/nrg3185 (2012).

20 Jaensson, E. et al. Small intestinal CD103+ dendritic cells display unique functional properties that are conserved between mice and humans. The Journal of experimental medicine 205, 21392149, doi:10.1084/jem.20080414 (2008).

21 Randolph, G. J. Emigration of monocyte-derived cells to lymph nodes during resolution of inflammation and its failure in atherosclerosis. Current opinion in lipidology 19, 462-468, doi:10.1097/MOL.0b013e32830d5f09 (2008).

22 Conus, S. et al. Caspase-8 is activated by cathepsin D initiating neutrophil apoptosis during the resolution of inflammation. The Journal of experimental medicine 205, 685-698, doi:10.1084/jem.20072152 (2008).

23 Fischbeck, A. et al. Sphingomyelin induces cathepsin D-mediated apoptosis in intestinal epithelial cells and increases inflammation in DSS colitis. Gut 60, 55-65, doi:10.1136/gut.2009.201988 (2011).

24 Menzel, K. et al. Cathepsins B, L and D in inflammatory bowel disease macrophages and potential therapeutic effects of cathepsin inhibition in vivo. Clinical and experimental immunology 146, 169-180, doi:10.1111/j.1365-2249.2006.03188.x (2006).

$25 \mathrm{Li}, \mathrm{W}$. \& Yuan, X. M. Increased expression and translocation of lysosomal cathepsins contribute to macrophage apoptosis in atherogenesis. Annals of the New York Academy of Sciences 1030, 427-433, doi:10.1196/annals.1329.053 (2004).

26 Figueiredo, J. L. et al. Selective cathepsin S inhibition attenuates atherosclerosis in apolipoprotein E-deficient mice with chronic renal disease. Am J Pathol 185, 1156-1166, doi:10.1016/j.ajpath.2014.11.026 (2015).

27 Maxfield, F. R. \& Tabas, I. Role of cholesterol and lipid organization in disease. Nature 438, 612-621, doi:10.1038/nature04399 (2005).

$28 \mathrm{Xu}, \mathrm{X}$. et al. Obesity activates a program of lysosomal-dependent lipid metabolism in adipose tissue macrophages independently of classic activation. Cell metabolism 18, 816-830, doi:10.1016/j.cmet.2013.11.001 (2013). 


\section{Chapter 3}

29 Murphy, J., Summer, R., Wilson, A. A., Kotton, D. N. \& Fine, A. The prolonged life-span of alveolar macrophages. Am J Respir Cell Mol Biol 38, 380-385, doi:10.1165/rcmb.20070224RC (2008).

30 Fucho, R. et al. ASMase regulates autophagy and lysosomal membrane permeabilization and its inhibition prevents early stage non-alcoholic steatohepatitis. J Hepatol 61, 1126-1134, doi:10.1016/j.jhep.2014.06.009 (2014).

31 Xiong, J. \& Kielian, T. Microglia in juvenile neuronal ceroid lipofuscinosis are primed toward a pro-inflammatory phenotype. Journal of neurochemistry 127, 245-258, doi:10.1111/jnc.12385 (2013).

32 Siintola, E. et al. Cathepsin D deficiency underlies congenital human neuronal ceroidlipofuscinosis. Brain : a journal of neurology 129, 1438-1445, doi:10.1093/brain/awl107 (2006).

33 Pullinger, C. R. et al. Human cholesterol 7alpha-hydroxylase (CYP7A1) deficiency has a hypercholesterolemic phenotype. J Clin Invest 110, 109-117, doi:10.1172/JCI15387 (2002).

34 Wang, L. et al. Redundant pathways for negative feedback regulation of bile acid production. Developmental cell 2, 721-731 (2002).

35 Watanabe, M. et al. Bile acids lower triglyceride levels via a pathway involving FXR, SHP, and SREBP-1c. J Clin Invest 113, 1408-1418, doi:10.1172/JCI21025 (2004).

36 Jonkers, I. J., Mohrschladt, M. F., Westendorp, R. G., van der Laarse, A. \& Smelt, A. H. Severe hypertriglyceridemia with insulin resistance is associated with systemic inflammation: reversal with bezafibrate therapy in a randomized controlled trial. The American journal of medicine $112,275-280$ (2002).

37 Guicciardi, M. E., Malhi, H., Mott, J. L. \& Gores, G. J. Apoptosis and necrosis in the liver. Compr Physiol 3, 977-1010, doi:10.1002/cphy.c120020 (2013).

38 Settembre, C. et al. TFEB links autophagy to lysosomal biogenesis. Science 332, 1429-1433, doi:10.1126/science.1204592 (2011).

39 Rosenfeld, M. G., Kreibich, G., Popov, D., Kato, K. \& Sabatini, D. D. Biosynthesis of lysosomal hydrolases: their synthesis in bound polysomes and the role of co- and posttranslational processing in determining their subcellular distribution. The Journal of cell biology 93, 135-143 (1982).

40 Hoppe, G., O'Neil, J., Hoff, H. F. \& Sears, J. Products of lipid peroxidation induce missorting of the principal lysosomal protease in retinal pigment epithelium. Biochim Biophys Acta 1689, 33-41, doi:10.1016/j.bbadis.2004.01.004 (2004).

41 Bieghs, V. et al. Role of scavenger receptor A and CD36 in diet-induced nonalcoholic steatohepatitis in hyperlipidemic mice. Gastroenterology 138, 2477-2486, 2486 e2471-2473, doi:10.1053/j.gastro.2010.02.051 (2010).

42 Wouters, K. et al. Dietary cholesterol, rather than liver steatosis, leads to hepatic inflammation in hyperlipidemic mouse models of nonalcoholic steatohepatitis. Hepatology 48, 474-486, doi:10.1002/hep.22363 (2008). 


\section{Chapter 3}

\section{Supplemental figures}

\section{Ldl/'/- mice}

3 weeks

CHOW

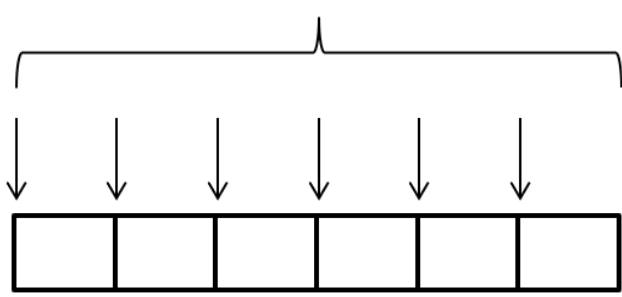

Control

Pepstatin A

HFC

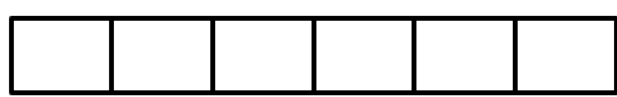

HFC

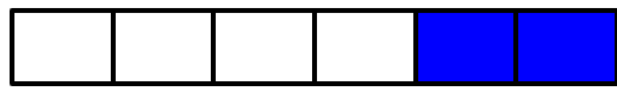

Supplemental Figure 1: Overview of all experimental groups of the in vivo experiment. Arrows indicate time of i.p. injection. 


\section{Chapter 3}
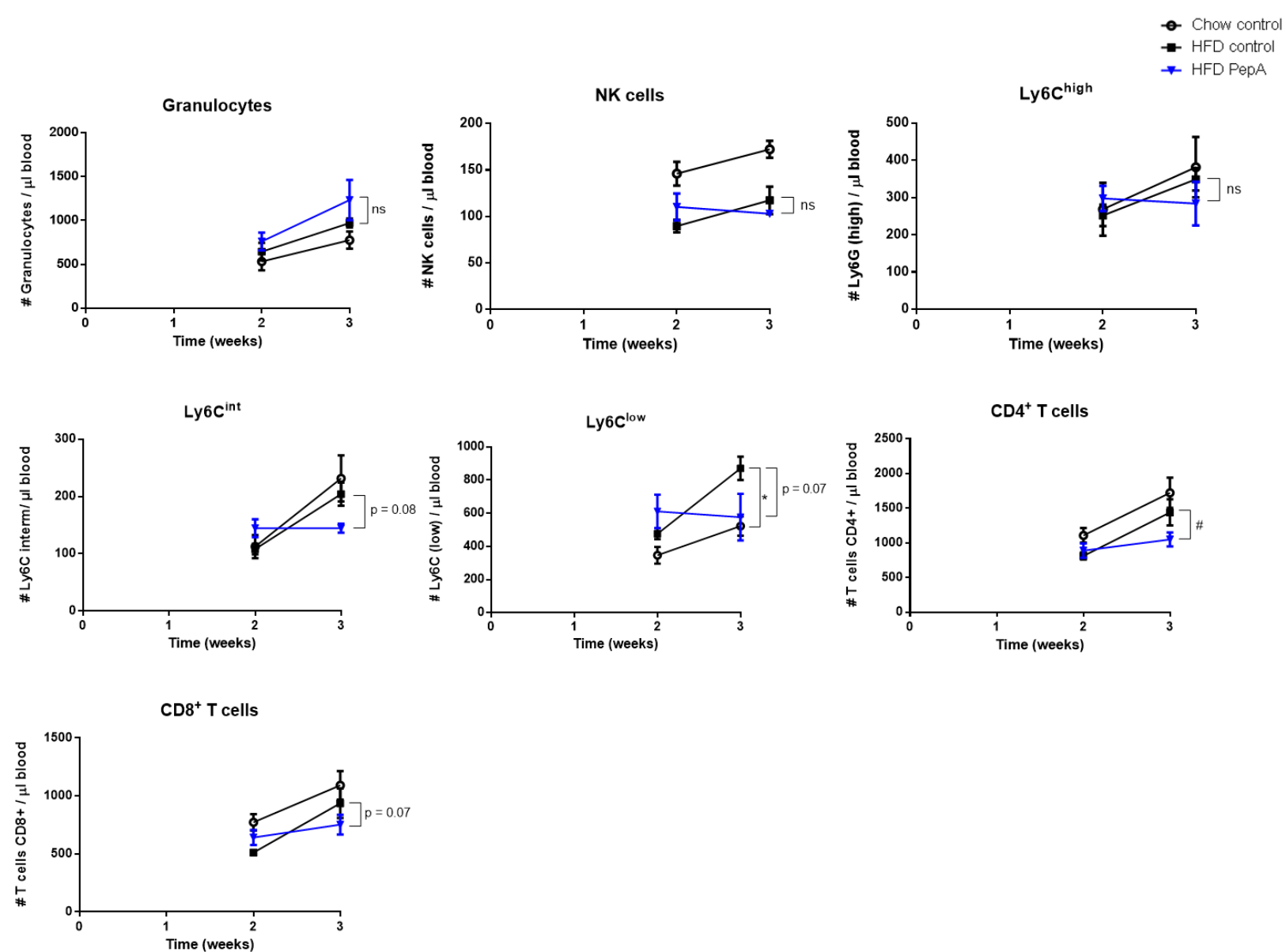

Supplemental Figure 2: Blood leukocyte profile of granulocytes, NK cells, the monocyte subpopulations (Ly6Chigh, Ly6Cint and Ly6Clow) and T cell subpopulations (T-helper (CD4) and cytotoxic T cell (CD8)) by use of two-tailed unpaired $t$ test. Error bars represent \pm SEM. * Indicates $\mathrm{p}<0.05$ compared to mice on chow diet and $\# \mathrm{p}<0.05$ compared to control-injected mice on HFC diet. $\mathrm{n}=4$ animals per group. ns, not significant. 


\section{Chapter 3}

A

Chow - Control

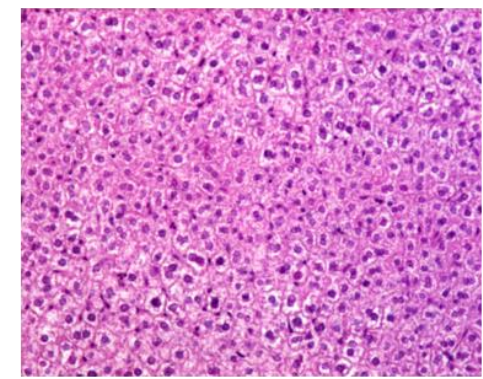

HFC - Control

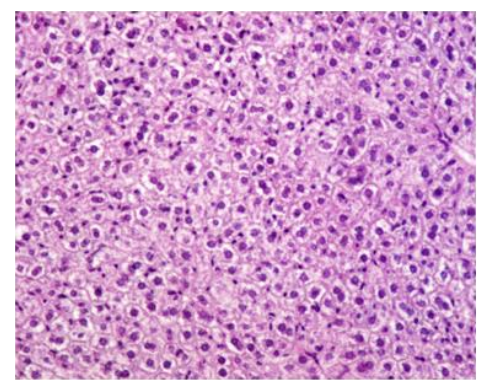

HFC - PepA

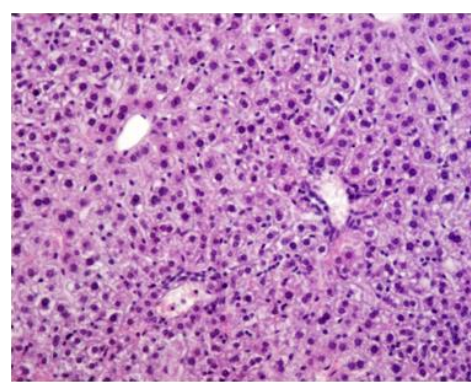

B

Inflammation

$\square$ Chow control
HFC control
HFC PepA (1w)

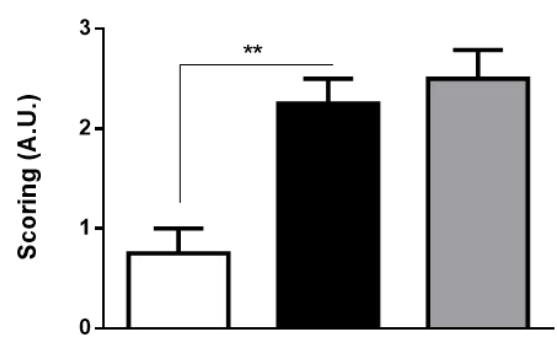

C

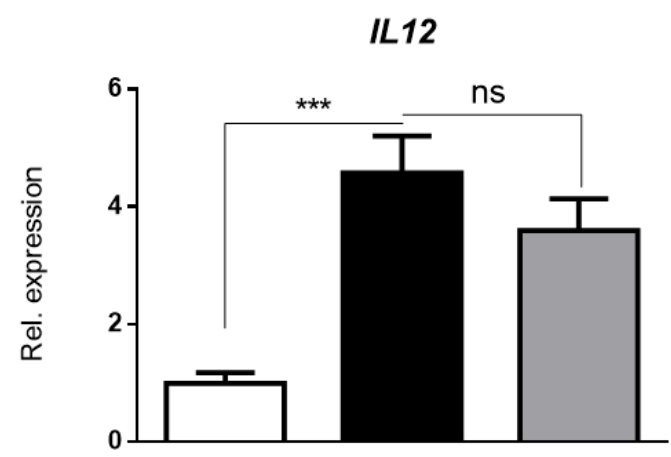

\section{Vcam}

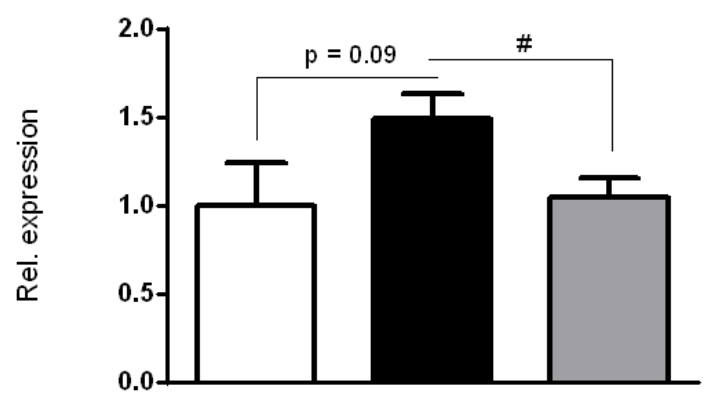

Supplemental Figure 3: (A) General histology of the liver by a hematoxylin and eosin staining of control- and PepA-injected mice on a chow or HFC diet (original magnification, 200x). (B) Scoring for inflammation of H\&E staining. (C) Hepatic gene expression analysis of IL12 and Vcam. Data are shown relative to control mice on chow diet by use of two-tailed unpaired t. Error bars represent \pm SEM. * Indicates $\mathrm{p} \leq 0.05,{ }^{* *} \mathrm{p}<0.01$ and ${ }^{* * *} \mathrm{p}<0.001$ compared to mice on chow diet; \# indicates $\mathrm{p}<0.05$ compared to control-injected mice on HFC diet. $\mathrm{n}=8-11$ animals per group. $\mathrm{ns}$, not significant. 


\section{Chapter 3}

$\square$ Chow control

HFC control

HFC PepA (1w)

A

B

Hepatic CTSE activity
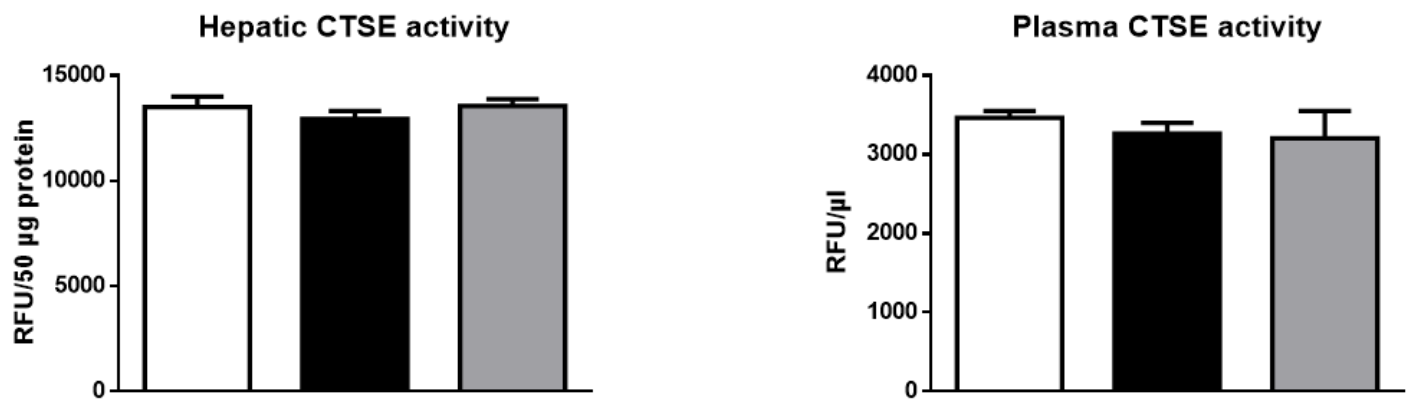

C

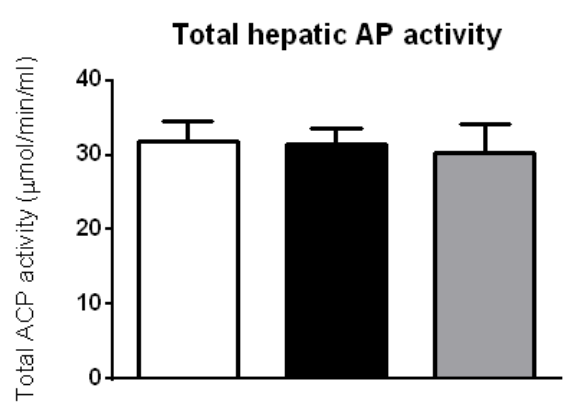

D

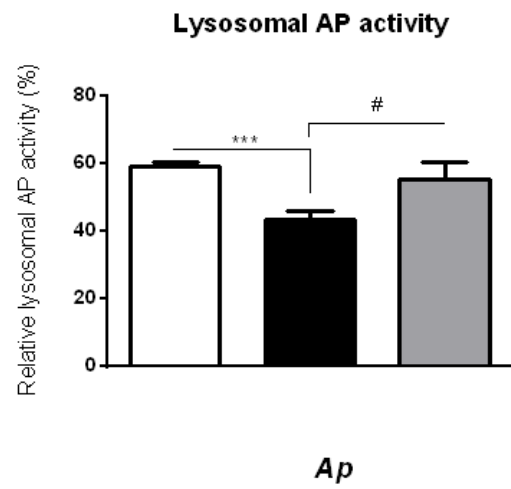

E

Ctss

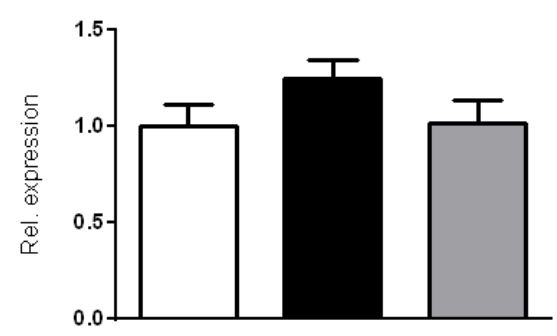

Supplemental Figure 4: (A, B) Hepatic (A) and plasma (B) activity of CTSE. (C, D)) Total and lysosomal activity of the lysosomal enzyme acid phosphatase (AP) in the liver of control- and PepA-injected mice. (E) Hepatic gene expression analysis of Ctss and Ap. Data are shown relative to control mice on chow diet by use of two-tailed unpaired $t$ test. Error bars represent \pm SEM. *** Indicates $p<0.001$ compared to mice on chow diet; \# indicates $\mathrm{p} \leq 0.05$ compared to control-injected mice on HFC diet by use of two-tailed unpaired $\mathrm{t}$ test. $\mathrm{n}=9-11$ animals in each group. 


\section{Chapter 3}

A

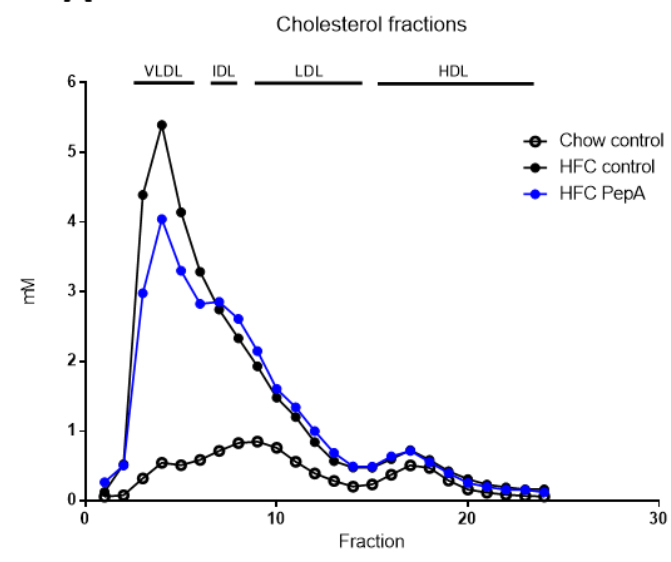

C

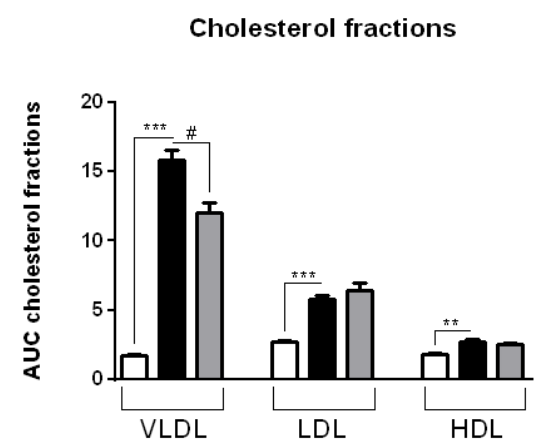

$\mathbf{E}$

Intracellular lipid accumulation

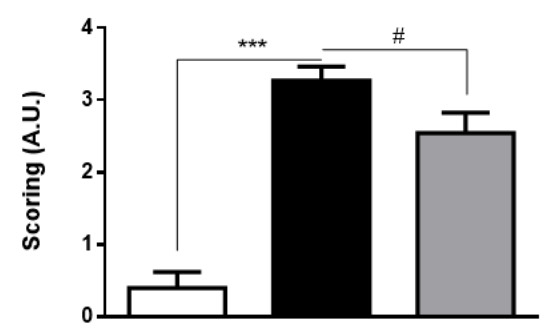

B

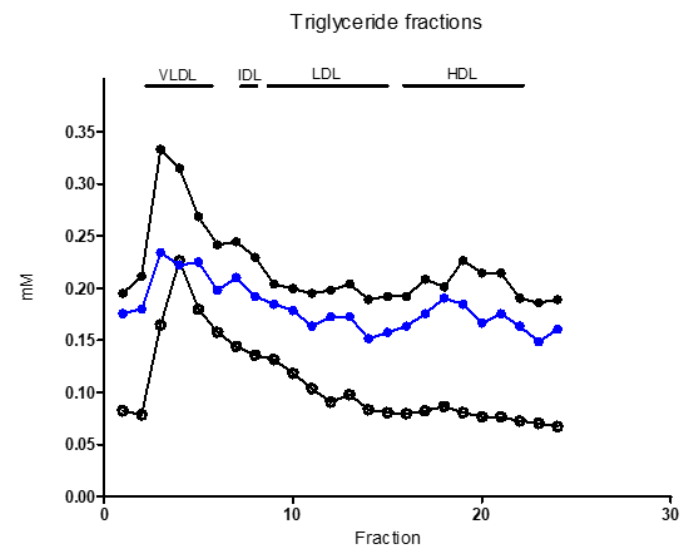

D

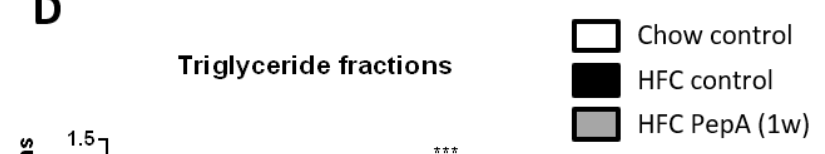

$\mathbf{F}$

Relative liver weight

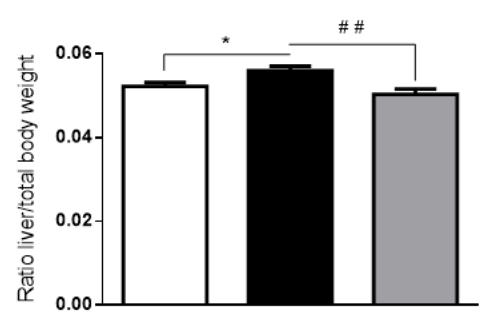

Supplemental Figure 5: (A, B) Using FPLC, plasma lipid and lipoprotein profiles were analyzed in all experimental groups. Sera of mice were pooled, creating $n=4$ for each experimental condition. On the chromatogram, the $\mathrm{X}$-axis represents the fractions present in the mixture as a peak, thereby identifying the different components of the mixture. On the $\mathrm{Y}$-axis, the amount of the different fractions can be read (mM). (C, D) Quantification of plasma cholesterol (C) and triglycerides (D) fractions. (E) Quantification of the Oil Red O staining. (F) Relative liver weight of control- and PepA-injected mice on a chow or HFC diet. * Indicates $p<0.05$, ** $p<0.01$ and *** $p<0.001$ compared to mice on chow diet; \# indicates $\mathrm{p}<0.05$ and \#\# indicates $\mathrm{p}<0.01$ compared to control-injected mice on HFC diet by use of the two-tailed unpaired $t$ test. Error bars represent \pm SEM. 


\section{Chapter 3}

A

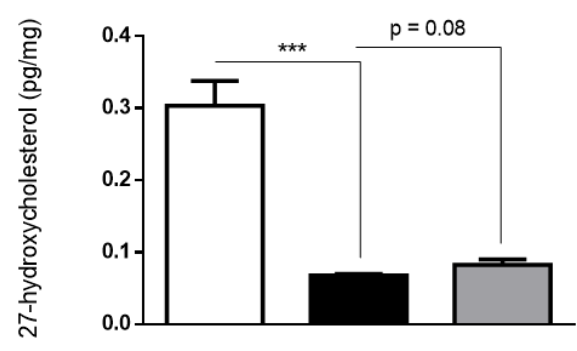

B

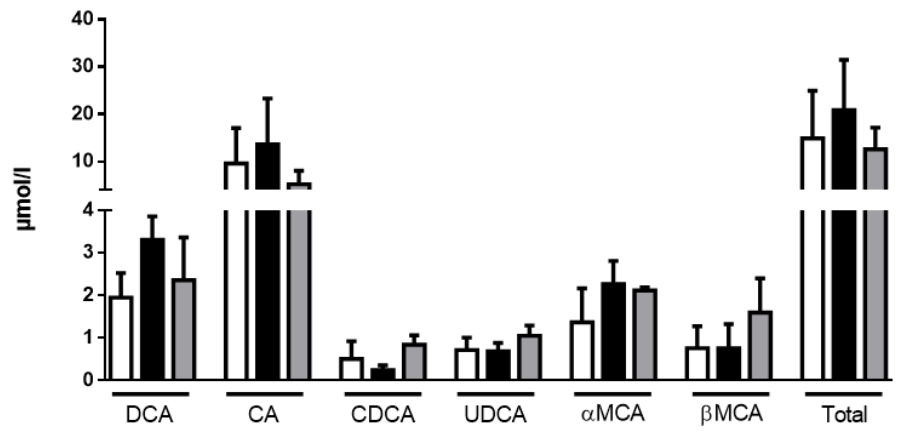

$\square$ Chow control

HFC control

HFC PepA (1w)

C

Total hepatic bile acids

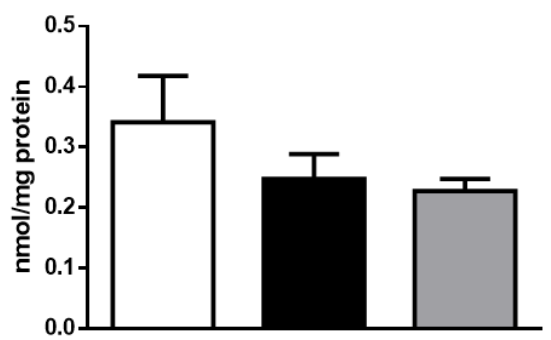

Supplemental Figure 6: (A) Hepatic concentration of 27-hydroxycholesterol (27HC) by use of two-tailed unpaired t. (B) Plasma bile acid levels. (C) Total hepatic bile acid levels. Error bars represent \pm SEM. *** Indicates $\mathrm{p}<0.001$ compared to mice on chow diet. Plasma bile acid levels data are pooled from 11 mice, thereby creating $\mathrm{n}=4$ for each experimental group; $\mathrm{n}=9-11$ animals in each group. DCA, deoxycholic acid; CA, cholic acid; CDCA, chenodeoxycholic acid; UDCA, ursodeoxycholic acid; $\alpha$ MCA, $\alpha$-muricholic acid; $\beta$ MCA, $\beta$ muricholic acid. 


\section{Chapter 3}

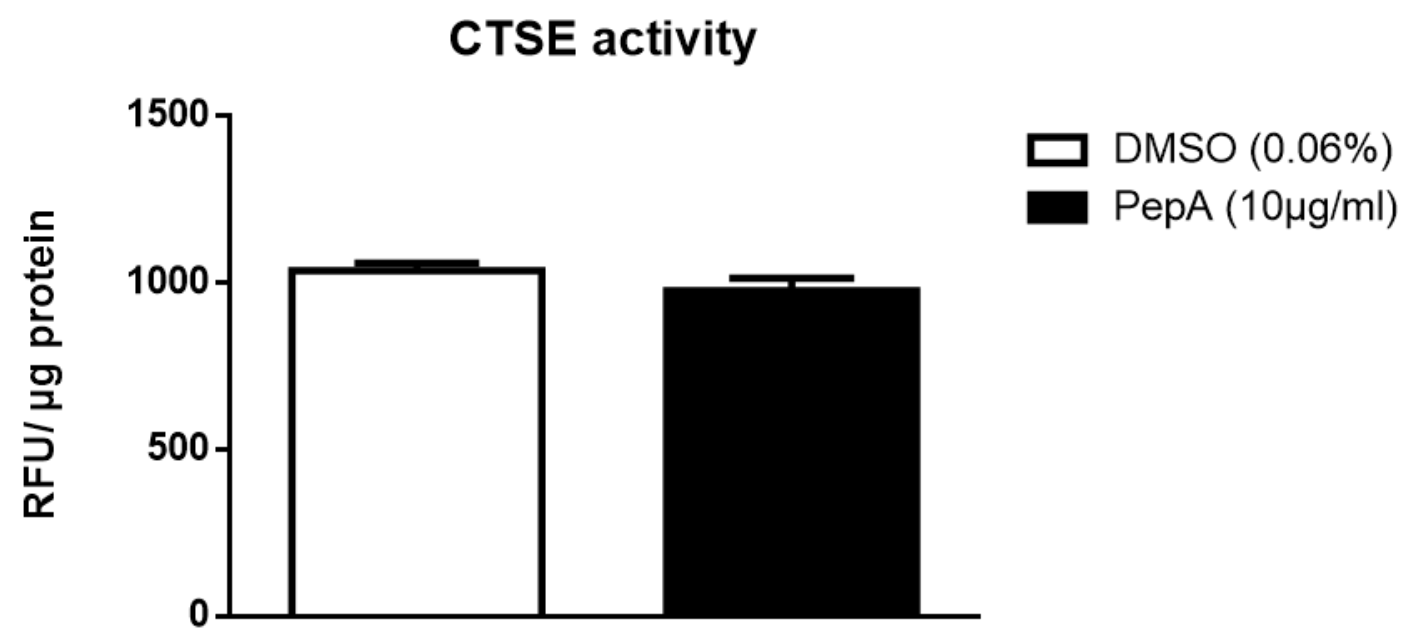

Supplemental Figure 7: CTSE activity in BMDMs. Error bars represent $\pm \operatorname{SEM}(\mathrm{n}=3)$ 


\section{Chapter 3}

Supplementary Table S1: Hepatic inflammation after 1 or 3 weeks of pepstatin A treatment

\begin{tabular}{|c|c|c|c|c|}
\hline & Chow & & HFC & \\
\hline & Control & Control & $\operatorname{PepA}(1 w)$ & PepA (3w) \\
\hline \multicolumn{5}{|l|}{ Histology } \\
\hline $\begin{array}{l}\text { Infiltrating macrophages and } \\
\text { neutrophils (Macl) } \\
\text { (\# positive cells } / \mathrm{mm}^{2} \text { ) }\end{array}$ & $32.58 \pm 5.191$ & $122.7 \pm 11.71 * * *$ & $124.6 \pm 13.33$ & $124.8 \pm 6.16$ \\
\hline $\begin{array}{l}T \text { cells }(C D 3) \\
\left(\# \text { positive cells } / \mathrm{mm}^{2}\right)\end{array}$ & $71.88 \pm 9.59$ & $130.6 \pm 22.61^{*}$ & $132.8 \pm 13.85$ & $74.77 \pm 7.77 \mathbf{a}$ \\
\hline $\begin{array}{l}\text { Monocytes/macrophages (CD68) } \\
\text { (A.U.) }\end{array}$ & $1.4 \pm 0.22$ & $3.5 \pm 0.31 * * *$ & $3.00 \pm 0.33$ & $3.00 \pm 0.26$ \\
\hline $\begin{array}{l}\text { Macrophage }(F 4 / 80) \\
(\% \text { positive area })\end{array}$ & $1.1 \pm 0.21$ & $1.3 \pm 0.3$ & $1.3 \pm 0.2$ & $0.9 \pm 0.13$ \\
\hline \multicolumn{5}{|l|}{ Gene expression (Rel. Exp.) } \\
\hline $\operatorname{Tnf} \alpha$ & $1.00 \pm 0.12$ & $9.52 \pm 1.27 * * *$ & $5.89 \pm 0.91 \#$ & $5.25 \pm 0.86 \#$ \\
\hline Caspase1 & $1.00 \pm 0.10$ & $1.98 \pm 0.14 * * *$ & $1.45 \pm 0.14 \#$ & $1.36 \pm 0.11 \# \#$ \\
\hline Ccl2 & $1.00 \pm 0.14$ & $3.724 \pm 0.29 * * *$ & $2.00 \pm 0.33 \# \# \#$ & $1.87 \pm 0.47 \# \#$ \\
\hline Cd68 & $1.00 \pm 0.08$ & $3.98 \pm 0.22 * * *$ & $2.77 \pm 0.20 \# \# \#$ & $2.52 \pm 0.34 \# \#$ \\
\hline Ill2 & $1.00 \pm 0.18$ & $4.57 \pm 0.63 * * *$ & $3.60 \pm 0.54$ & $2.55 \pm 0.35 \#$ \\
\hline Vcam & $1.00 \pm 0.24$ & $1.50 \pm 0.14$ & $1.05 \pm 0.11 \#$ & $1.01 \pm 0.15 \#$ \\
\hline$C d 206$ & $1.00 \pm 0.09$ & $1.27 \pm 0.12$ & $1.03 \pm 0.15$ & $0.96 \pm 0.12 \mathbf{b}$ \\
\hline Egr2 & $1.00 \pm 0.18$ & $7.06 \pm 0.77 * * *$ & $4.48 \pm 0.53 \#$ & $3.87 \pm 0.77 \# \#$ \\
\hline iNos/Argl & $1.00 \pm 0.3$ & $7.37 \pm 1.1 * * *$ & $6.13 \pm 1.23$ & $5.44 \pm 0.82$ \\
\hline \multicolumn{5}{|l|}{ Protein levels (pg/mg protein) } \\
\hline $\mathrm{TNF} \alpha$ & $38.94 \pm 4.19$ & $58.37 \pm 11.20$ & $24.62 \pm 3.28 \# \#$ & $18.42 \pm 2.27$ \#\# \\
\hline IL12 & $0.03 \pm 0.01$ & $0.12 \pm 0.04 *$ & $0.02 \pm 0.01 \#$ & $0.02 \pm 0.01 \#$ \\
\hline
\end{tabular}

*Indicates $p<0.05$ and $* * * p<0.001$ compared to mice on chow diet; \# indicates $p \leq 0.05$, \#\# $p<0.01$ and \#\#\# $p<0.001$ compared to control-injected mice on HFC diet; a indicates $\mathrm{p}=0.06$ and $\mathrm{b}$ indicates $\mathrm{p}=0.09$ compared to control-injected mice on HFC diet by use of twotailed unpaired $t$ test. Standards of error represent \pm SEM. 


\section{Chapter 3}

\section{Supplementary Methods}

\section{Acid phosphatase activity assay}

Hepatic acid phosphatase (AP) activity was determined by the acid phosphatase assay kit (10008051, Cayman Chemical Company, USA). Liver homogenates were diluted 20 times in assay buffer, and transferred to a 96-well plate containing $20 \mu \mathrm{l}$ assay buffer. Next, $20 \mu \mathrm{l}$ AP substrate solution was added to each well to initiate the reaction. After 20 minutes incubation at $37^{\circ} \mathrm{C}$, the reaction was stopped by adding $100 \mu \mathrm{l}$ of stop solution to all wells. The absorbance was measured using a Bio-Rad Benchmark 550 Micro-plate reader at 405-414 nm (170-6750XTU, Bio-Rad Laboratories, Veenendaal, the Netherlands).

\section{Cathepsin D activity assay}

Cathepsin D activity was measured using the cathepsin D activity assay kit (MBL International, Woburn, MA) according to the manufacturer's protocol. In summary, $50 \mu \mathrm{g}$ of liver homogenate or 5 $\mu \mathrm{L}$ plasma was lysed in cathepsin D lysis buffer on ice for 10 minutes. Following centrifugation for 5 minutes at top speed, $5 \mu \mathrm{L}$ of clear cell lysate was transferred to a well of a 96-well plate, and the total volume was made up to $50 \mu \mathrm{L}$ with cathepsin D cell lysis buffer. To each assay, $52 \mu \mathrm{L}$ of mastermix (50 $\mu \mathrm{L}$ if CTSD Reaction Buffer and $2 \mu \mathrm{L}$ of CTSD Substrate) was added, and the plate was incubated at $37^{\circ} \mathrm{C}$ for 1 hour. Samples were then measured using a fluorescence plate reader with a $328-\mathrm{nm}$ excitation filter and 460-nm filter. Cathepsin D activity is expressed by the relative fluorescence units.

\section{Cathepsin E activity assay}

Cathepsin E activity was measured using the cathepsin E activity assay kit (BioVision, Milpitas, CA) according to the manufacturer's protocol. In summary, $50 \mu \mathrm{g}$ of liver homogenate, $5 \mu \mathrm{L}$ plasma or 50 $\mu \mathrm{g}$ bone marrow-derived macrophages was lysed in cathepsin $\mathrm{E}$ lysis buffer on ice for 5 minutes. Following centrifugation for 10 minutes at top speed, $5 \mu \mathrm{L}$ of clear cell lysate was transferred to a well of a 96-well plate, and the total volume was made up to $50 \mu \mathrm{L}$ with cathepsin E cell lysis buffer. To each assay, $50 \mu \mathrm{L}$ of Substrate mix (50 $\mu \mathrm{L}$ if CTSE Assay Buffer and $2 \mu \mathrm{L}$ of CTSE Substrate) was added, and the plate was incubated at $37^{\circ} \mathrm{C}$ for 1 hour. Samples were then measured using a fluorescence plate reader with a 320-nm excitation filter and 420-nm filter. Cathepsin E activity is expressed by the relative fluorescence units.

\section{Plasma Lipid Analysis}

Both plasma and liver lipid levels were measured with enzymatic color tests (cholesterol CHOD-PAP; 1489232; Roche, Basel, Switzerland; serum triglyceride (TG) determination kit, TR0100; SigmaAldrich; NEFAC, ACS-ACOD, 999-75406; Wako Chemicals, Neuss, Germany) according to the 


\section{Chapter 3}

manufacturer's protocols on a Benchmark 550 Micro-plate Reader (170-6750XTU; Bio-Rad, Hercules, CA).

\section{Liver Lipid Analysis}

Approximately $50 \mathrm{mg}$ of frozen liver tissue was homogenized for 30 seconds at $5000 \mathrm{rpm}$ in a closed tube with 1.0-mm glass beads and $1.0 \mathrm{~mL}$ SET buffer (sucrose $250 \mathrm{mmol} / \mathrm{L}$, EDTA $2 \mathrm{mmol} / \mathrm{L}$, and Tris $10 \mathrm{mmol} / \mathrm{L}$ ). Complete cell destruction was done by 2 freeze-thaw cycles and 3 times passing through a 27-gauge syringe needle and a final freeze-thaw cycle. Protein content was measured with the bicinchoninic acid (BCA) method (23225; Pierce, Rockford, IL). Cholesterol and triglycerides (TG) were measured as described above. Protocols were followed according to the manufacturers' instructions.

\section{Fluorescence-activated cell sorting}

FACS analysis was performed as previously described 1 . Graphs shown show the absolute values after 3 weeks (T3) and 2 weeks (T2).

\section{Lipoprotein profiles}

Lipoprotein profiles were determined on plasma samples from 4 mice (pooled from 11 to 4 mice of the same experimental group) and determined as described previously 2.

\section{Enzyme-linked immunosorbent assay (ELISA)}

TNF $\alpha$, IL10 and IL12 ELISA assays (resp. 88-7324-88, 88-7105-88 and 88-7121-88, eBioscience, Frankfurt, Germany) were performed on supernatant or liver homogenates according to manufacturer's instructions. Analysis was performed on a Bio-Rad Benchmark 550 Micro-plate reader $450 \mathrm{~nm}$.

\section{Measuring cholesterol precursors, oxysterols and bile acids}

Cholesterol precursors, oxysterols and bile acids were measured via highly specific and sensitive gas chromatography-mass spectrometry as described previously 3-5. Fecal measurement was performed on feces isolated at sacrifice. Total hepatic bile acid levels were specifically determined as described previously 6 . 
Primer Sequences for Quantitative PCR

\begin{tabular}{|l|l|l|}
\hline Gene & Primer forward & Primer reverse \\
\hline TNF $\alpha$ & CATCTTCTCAAAATTCGAGTGACAA & TGGGAGTAGACAAGGTACAACCC \\
\hline CCL2 & GCTGGAGAGCTACAAGAGGATCA & TCTCTCTTGAGCTTGGTGACAAAA \\
\hline Caspase1 & GGGACCCTCAAGTTTTGCC & GACGTGTACGAGTGGTTGTATT \\
\hline CD68 & TGACCTGCTCTCTCTAAGGCTACA & TCACGGTTGCAAGAGAAACATG \\
\hline CD36 & GCCAAGCTATTGCGACATGA & AAAAGAATCTCAATGTCCGAGACTTT \\
\hline LXR $\alpha$ & CGGAGCCCCTGCACTTC & ACAGGGATCGGTGGGACAT \\
\hline ABCA1 & CCCAGAGCAAAAAGCGACTC & GGTCATCATCACTTTGGTCCTTG \\
\hline PPARY & TCGCTGATGCACTGCCTATG & GAGAGGTCCACAGAGCTGATT \\
\hline HmG-CoAR & ACCATGCCATCGATAGAGATAGGA & CGTGCGTTTTCTCCAGGATT \\
\hline Cyp7a1 & CATTACAGAGTGCTGGCCAAGA & CGCAGAGCCTCCTTGATGAT \\
\hline Cyp27 & CTGCACTTCCTGCTGACCAAT & AGGGCCCATGTCAGTGTGTT \\
\hline IL12 & GGAACTACACAAGAACGAGAG & AAGTCCTCATAGATGCTACCA \\
\hline VCAM & GTGTTGAGCTCTGTGGGTTTTG & TTAATTACTGGATCTTCAGGGAATGAG \\
\hline CTSD & CCTCCATTCATTGCAAGATACTTG & CACATAGGTGCTGGACTTGTCACT \\
\hline CTSS & AAAGATTACTGGCTTGTGAAAAACAG & GCAATTCCGCAGTGATTTTTATT \\
\hline AP & GACCCTAATGGCAACTACCTCTCA & GGGAGCTTGCTTCCCATTG \\
\hline CD206 & TGCAAAGGACTGAAAGGAAACC & CCAGTCCAGGCATTGAAAGTG \\
\hline EGR2 & CTACCCGGTGGAAGACCTC & AATGTTGATCATGCCATCTCC \\
\hline iNOS & GCAAACCCAAGGTCTACGTTCA & CCTCATTGGCCAGCTGCTT \\
\hline Arg1 & CATGGGCAACCTGTGTCCTT & CGATGTCTTTGGCAGATATGCA \\
\hline
\end{tabular}

1 Hendrikx, T. et al. Bone marrow-specific caspase-1/11 deficiency inhibits atherosclerosis development in Ldlr(-/-) mice. FEBS J 282, 2327-2338, doi:10.1111/febs.13279 (2015).

2 Plat, J. et al. Protective role of plant sterol and stanol esters in liver inflammation: insights from mice and humans. PLoS One 9, e110758, doi:10.1371/journal.pone.0110758 (2014).

3 Lutjohann, D. et al. Profile of cholesterol-related sterols in aged amyloid precursor protein transgenic mouse brain. J Lipid Res 43, 1078-1085 (2002). 


\section{Chapter 3}

4 Schierwagen, R. et al. Seven weeks of Western diet in apolipoprotein-E-deficient mice induce metabolic syndrome and non-alcoholic steatohepatitis with liver fibrosis. Sci Rep 5, 12931, doi:10.1038/srep12931 (2015).

5 Czubayko, F., Beumers, B., Lammsfuss, S., Lutjohann, D. \& von Bergmann, K. A simplified micro-method for quantification of fecal excretion of neutral and acidic sterols for outpatient studies in humans. J Lipid Res 32, 1861-1867 (1991).

6 Modica, S., Murzilli, S. \& Moschetta, A. Characterizing Bile Acid and Lipid Metabolism in the Liver and Gastrointestinal Tract of Mice. Curr Protoc Mouse Biol 1, 289-321, doi:10.1002/9780470942390.mo100226 (2011). 


\section{Chapter 4}

Sex-opposed inflammatory effects of 27-

hydroxycholesterol are mediated via estrogen signaling 


\section{Chapter 4}

Chapter 4 Sex-opposed inflammatory effects of 27-hydroxycholesterol are mediated via estrogen signaling

Houben T*, Bitorina AV*, Oligschlaeger Y, Jeurissen MLJ, Sander Rensen, Köhler SE, Westerterp M, Lütjohann D, Theys J, Romano A, Plat J, Shiri-Sverdlov R

*Contributed equally

J Pathol 2020 Aug;251(4):429-439 


\title{
Chapter 4
}

\begin{abstract}
Despite the increased awareness of differences in the inflammatory response between men and women, only limited research has focused on the biological factors underlying these sex differences. The cholesterol derivative 27 -hydroxycholesterol $(27 \mathrm{HC})$ has been shown to have opposite inflammatory effects in independent experiments using mouse models of atherosclerosis and non-alcoholic steatohepatitis (NASH), pathologies characterized by cholesterol-induced inflammation. As the sex of these in vivo models differed, we hypothesized that $27 \mathrm{HC}$ exerts opposite inflammatory effects in males compared to females. To explore whether the sex-opposed inflammatory effects of $27 \mathrm{HC}$ translated to humans, plasma $27 \mathrm{HC}$ levels were measured and correlated with hepatic inflammatory parameters in obese individuals. To investigate whether $27 \mathrm{HC}$ exerts sex-opposed effects on inflammation, we injected $27 \mathrm{HC}$ to female and male Niemann-Pick disease type $\mathrm{C} 1$ mice (Npc1nih) that were used as an extreme model of cholesterol-induced inflammation. Finally, the involvement of estrogen signaling in this mechanism was studied in bone marrow-derived macrophages (BMDMs) that were treated with 27HC and 17ß-estradiol (E2). Plasma 27HC levels showed opposite correlations with hepatic inflammatory indicators between female and male obese individuals. Likewise, hepatic 27HC levels oppositely correlated between female and male Npc1nih mice. Twenty-seven hydroxycholesterol injections reduced hepatic inflammation in female Npc1nih mice in contrast to male Npc1nih mice, which showed increased hepatic inflammation after 27HC injections. Furthermore, 27HC administration also oppositely affected inflammation in female and male BMDMs cultured in E2enriched medium. Remarkably, female BMDMs showed higher ER $\alpha$ expression compared to male BMDMs. Our findings identify that the sex-opposed inflammatory effects of $27 \mathrm{HC}$ are E2-dependent and are potentially related to differences in ER $\alpha$ expression between females and males. Hence, the individual's sex needs to be taken into account when $27 \mathrm{HC}$ is employed as therapeutic tool as well as in macrophage estrogen research in general.
\end{abstract}




\section{Chapter 4}

\section{Introduction}

An increasing amount of evidence has pointed towards sex differences influencing physiological processes, including inflammatory responses [1-5]. In spite of these physiological differences between men and women, only limited research has focused on the biological factors underlying these differences. As gender-based prevention and therapy, as opposed to the 'one-size-fits all' approach, is expected to benefit both male and female patients [5], it is essential to improve our understanding on these underlying biological factors involved with sex differences.

Metabolically-induced inflammatory responses (referred to as metabolic inflammation or metaflammation) such as cholesterol-induced activation of macrophages [6,7] have been generally accepted as a cause for the chronic low-grade inflammatory state observed in obese patients [8]. Metaflammation is also responsible for the steep increase in the amount of obese individuals suffering from hepatic inflammation [9], identifying the liver as one of the most sensitive organs for cholesteroland lipid-induced metaflammation [10]. Relevantly, cholesterol-derived lipids known as oxysterols have been linked to obesity-associated inflammatory conditions [11,12]. The most abundant oxysterol in the human body is 27-hydroxycholesterol (27HC) [13], which is generated via enzymatic oxidation of cholesterol by sterol 27-hydroxylase (CYP27A1) [14]. Due to its cholesterol-expelling effects [15], $27 \mathrm{HC}$ has been investigated as a therapeutic agent in atherosclerosis and non-alcoholic steatohepatitis (NASH), pathologies characterized by cholesterol-induced macrophage activation leading to inflammation [7,16-19]. Whereas hematopoietic overexpression of Cyp27a1 [20] and subcutaneous administration of 27HC in female hyperlipidemic mice [21] resulted in reduced hepatic inflammation, subcutaneous injections of $27 \mathrm{HC}$ in a comparable male hyperlipidemic mouse model paradoxically elevated atherosclerosis via increasing pro-inflammatory processes [22]. These results suggest that sex differences may underlie the opposite effects of $27 \mathrm{HC}$ on cholesterol-induced inflammation.

Based on these findings, we hypothesized that under cholesterol-induced inflammatory conditions, 27HC exerts sex-opposed inflammatory effects. Firstly, we found in a cohort of obese individuals that higher plasma 27HC levels in females corresponded with lower hepatic inflammatory indicators, while the opposite was observed in males. Similar sex-opposed inflammatory effects of $27 \mathrm{HC}$ were observed in Niemann-Pick nih mice (Npc1nih), here used as an extreme model mimicking cholesterol-induced inflammation [23]. Finally, in vitro experiments with murine bone marrow-derived macrophages (BMDMs) provided evidence that the sex-opposed inflammatory effect of 27HC is E2-dependent and is potentially related to differences in ER $\alpha$ expression between females and males. Altogether, these 


\section{Chapter 4}

data demonstrate for the first time the sex-opposed effects of $27 \mathrm{HC}$ on inflammation. Hence, the individual's sex needs to be taken into account when $27 \mathrm{HC}$ is employed as therapeutic tool.

\section{Methods}

\section{Human cohorts}

\section{Maastricht cohort}

43 obese adult individuals ( 21 females and 13 males respectively) undergoing bariatric surgery at the Maastricht University Medical Center or at the Atrium Medical Center Parkstad, Heerlen, the Netherlands, were included in the study. Population characteristics are represented in supplementary table S1A. NASH was determined via evaluation of liver biopsies based on Brunt's criteria by a trained pathologist. Exclusion criteria, the procedure of obtaining liver biopsies and blood sampling of this cohort have been previously described [24,25]. Determination of plasma 27HC levels was performed as previously described [21].

\section{$\underline{\text { Use of human subjects }}$}

The study was carried out in accordance with the approved guidelines by the Medical Ethical Committees of both the Maastricht University Medical Centre and the Atrium Medical Centre Parkstad and conducted in accordance with the revised version of the Declaration of Helsinki (October 2008, Seoul). Written informed consent was obtained from all the subjects.

\section{Mice, diet and treatment}

Experiments were performed according to the Dutch regulation and approved by the Committee for Animal Welfare of Maastricht University. Npc1nih mice were housed under standard conditions and were given free access to food and water. Npc1nih mice (a kind gift from Prof. Dr. Lieberman from University of Michigan Medical School) were derived from heterozygous founders (C57BL/6 / Npc1nih). In the first in vivo experiment, seven week-old female $(n=4)$ and male $(n=4)$ Npc1nih mice were given a normal chow diet and sacrificed. In the second in vivo experiment, two week-old female ( $\mathrm{n}=5 /$ group) and male ( $\mathrm{n}=5 /$ group) Npc1nih mice were daily injected with 27-hydroxycholesterol $(27 \mathrm{HC} ; 40 \mathrm{mg} / \mathrm{kg}$ ) or vehicle (2-hydroxypropyl- $\beta$-cyclodextrin $18 \%$ ) for 21 weeks and given a normal chow diet. Tissue specimens were isolated and snap-frozen in liquid nitrogen and stored at $-80^{\circ} \mathrm{C}$ or fixed in $4 \%$ formaldehyde/PBS. The collection of blood and hepatic and bile oxysterols was done as described previously $[19,26,27]$. Product information is provided in Supplementary Table S1. 


\section{Chapter 4}

\section{RNA isolation/cDNA synthesis/qRT-PCR}

Total RNA was isolated from mouse liver tissues or cultured bone marrow-derived macrophages (BMDMs). For liver tissues, homogenization was achieved by adding $1.0 \mathrm{~mm}$ of glass beads and 1.0 $\mathrm{ml}$ Tri Reagent (Sigma Aldrich, Saint Louis, USA) to the frozen liver tissues in a closed tube for 30 sec. at $4800 \mathrm{rpm}$. For BMDMs, $350 \mu \mathrm{l}$ of Tri Reagent was added to the well, after which BMDMs were transferred to a tube. After centrifugation with addition of chloroform $200 \mu \mathrm{l}$ for liver tissues and 100 $\mu \mathrm{l}$ for BMDMs), an aqueous phase was visible and transferred to a fresh tube. Isopropanol ( $0.5 \mathrm{ml})$ was added and upon another centrifugation step, RNA was precipitated. A wash step of the RNA pellet was done by adding $1.0 \mathrm{ml} \mathrm{70 \%} \mathrm{ethanol.} \mathrm{After} \mathrm{centrifugation,} \mathrm{the} \mathrm{supernatant} \mathrm{was} \mathrm{removed} \mathrm{and} \mathrm{the} \mathrm{pellet}$ was dissolved in an appropriate volume of DEPC sterile H2O. All materials used were RNAse free and samples were placed on ice during the procedure. Afterwards, the RNA concentration and RNA quality were determined on a NanoDrop ND-1000 spectrophotometer.

Total RNA (500 ng of the liver and $300 \mathrm{ng}$ of BMDMs) was reverse transcribed in first-strand complementary DNA (cDNA) by using the iScript cDNA synthesis kit according to the manufacturer's instructions. Changes in the expression of the indicated genes were determined by qPCR of $10 \mathrm{ng}$ cDNA on Bio-Rad MyIQ with IQ5 v2.1 software (Applied Biosystems ABI7900) using IQ SensiMix SYBR master mix with fluorescein (Bioline, London, UK). Cyclophillin A, Ribosomal protein S12 and betaactin were used as reference genes to standardize for the amount of cDNA. By using default settings in primer Express version 2.0 (Applied Biosystems, Forster City, CA, USA), primer sets for the selected genes were developed and are available in Supplementary Table S2. qPCR data was analyzed according to the relative standard curve method.

\section{Immunohistochemistry}

Frozen liver sections $(7 \mu \mathrm{m})$ were fixed in acetone and subsequently blocked for endogenous peroxidase by incubation with $0.25 \%$ of $0.03 \% \mathrm{H} 202$ for 5 minutes. Prior to the incubation with the first antibody, slides were incubated with $4 \%$ fetal calf serum/ 1x PBS + 1:5 Avidin D Block solution. Primary antibodies used were against infiltrated macrophages and neutrophils. For NIMP staining, no amplification step was needed and as a secondary antibody, $\alpha$-rat-PO was used. For the incubation step with the first antibody, we used 4\% FCS/ 1x PBS + 1st antibody + 1:5 Biotin Block solution (ABC kit). Incubation with the second antibody (1:300 $\alpha$-rat-BIO or 1:100 $\alpha$-rat-PO) was done in $4 \%$ FCS/2\% normal mouse serum/ 1x PBS. After this, the slides were washed and incubated in 1x PBS + 1:50 Biotin solution (ABC kit). Then, stainings were finished using the AEC kit (2\% buffer/ 3\% AEC/ $2 \% \mathrm{H} 2 \mathrm{O} 2$ in demi water, used as a color substrate and hematoxylin for nuclear staining. Sections were enclosed with Faramount aqueous mounting medium. 
Paraffin embedded liver sections $(4 \mu \mathrm{m})$ were stained with Hematoxylin-Eosin after deparaffination in xylol and dehydrated with resp. $90 \%, 70 \%$ and $50 \%$ ethanol. Subsequently, Coupes were mounted with Entallan. Pictures were taken with a Nikon DMX1200 digital camera and ACT-1 v2.63 software. Immune cells were counted in 6 microscopical views (original magnification, 200x) and were noted as cells/square millimeter.

\section{Cell culture}

BMDMs were isolated from the tibiae and femurs of male or female Npc1nih and Wt C57BL/6 mice. Cells were cultured for eight days in RPMI-1640 supplemented with 10\% heat-inactivated fetal calf serum, penicillin $(100 \mathrm{U} / \mathrm{ml})$, streptomycin $(100 \mu \mathrm{g} / \mathrm{ml})$ and L-glutamine $2 \mathrm{mM}$, supplemented with 20\% L929-conditioned medium (LCM) to generate BMDMs. After attachment, BMDMs were seeded at 350,000 cells per well in 24-well plates. A complete overview of the experiment is depicted in Supplementary figure S1. At the end of the experiment, medium was collected and cells were lysed for mRNA expression or protein analysis. All in vitro data represent at least $n=3$ (triplicates) for each experimental group.

\section{Nitrite assay}

Levels of nitric oxide (NO) were measured in medium using the Griess $(0.1 \%$ naphthylethylenediamine dihydrochloride; $1 \%$ sulfanilamide; $2.5 \%$ phosphoric acid) test protocol. Standard curve was made using dilutions of NaNO2 in E2 medium: $10 \mu 1,8.75 \mu 1,7.5 \mu 1,6.25 \mu 1,5 \mu 1,3.75 \mu 1$ (E2medium blank). $50 \mu \mathrm{l}$ of standard dilution and $45 \mu \mathrm{l}$ of samples $+5 \mu \mathrm{l}$ of $50 \mu \mathrm{M}$ NaNO2 were pipetted into a 96-well plate. $50 \mu 1$ of Griess agent was added and absorbance was measured at $545 \mathrm{~nm}$.

\section{Tumor necrosis factor alpha (TNFa), interleukin 10 (IL-10), estrogen receptor alpha (ER $\alpha$ ) and estrogen receptor beta (ERß), liver X receptor beta (LXRß) enzyme linked immunosorbent assays (ELISA)}

The mouse TNFa and IL-10 ELISA was performed on medium of bone-marrow derived macrophages according to manufacturer's instructions (Sensitivity: $8 \mathrm{pg} / \mathrm{ml}$ (TNFa) or $23 \mathrm{pg} / \mathrm{ml}$ (IL-10); Specificity: cross contamination from other specimens possible). Protein expression of ER $\alpha$ and ERß was measured on cell lysate of bone marrow-derived macrophages according to manufacturer's instructions (ER $\alpha$ Sensitivity: $0.19 \mathrm{ng} / \mathrm{ml}$ and ERß Sensitivity: $0.19 \mathrm{ng} / \mathrm{ml}$ ). No significant cross-reactivity or interference between ER $\alpha$ and ER $\beta$ was observed. LXR $\beta$ was measured on cell lysate of Wt bone marrow-derived macrophages according to manufacturer's instructions. 


\section{Chapter 4}

\section{Statistical analysis}

The differences between the experimental groups were analyzed with two-way ANOVA followed by a Tukey post-hoc test using the IBM® SPSS Statistics program (Version 22.0.0.) or GraphPad Prism (Version 6.01). The results were visualized and expressed as the mean \pm SEM and considered significant at $\mathrm{p} \leq 0.05, *$ and $* *$ indicate $\mathrm{p} \leq 0.01$ respectively using GraphPad Prism (Version 6.01). Pearson correlation coefficients ( $r$ ) and respective $p$ values were calculated to assess the statistical significance of the correlation.

\section{Results}

\section{Plasma 27-hydroxycholesterol levels are oppositely associated with hepatic inflammatory indicators between female and male obese individuals}

To explore whether sex associates with the inflammatory effect of $27 \mathrm{HC}$, plasma $27 \mathrm{HC}$ levels were measured in an obese population and correlated with different hepatic inflammatory indicators. Clinical characteristics of the population are presented in Supplementary Table S3. In the 'Maastricht cohort' $(n=43)$, plasma 27HC levels were significantly lower in female NASH individuals (categorized by criteria of Brunt) as compared to no-NASH obese females (Fig. 1A and Supplementary figure S2A, left panel). Conversely, in male NASH individuals, plasma 27HC levels were increased compared to noNASH obese males (Fig. 1A and Supplementary figure S2A, right panel). Similarly, when plasma 27HC levels were specifically compared with hepatic lobular inflammation in the same obese population, significantly lower plasma $27 \mathrm{HC}$ levels were observed in obese females suffering from hepatic lobular inflammation (Fig. 1B and Supplementary figure S2B, left panel), while an increasing trend was found for plasma 27HC levels in obese males (Fig. 1B and Supplementary figure S2B, right panel). These results show that plasma $27 \mathrm{HC}$ levels oppositely correlate with hepatic inflammatory indicators in female and male obese individuals, confirming the sex-opposed inflammatory association with 27HC that was previously observed in hyperlipidemic models [20-22]. 


\section{Chapter 4}

A
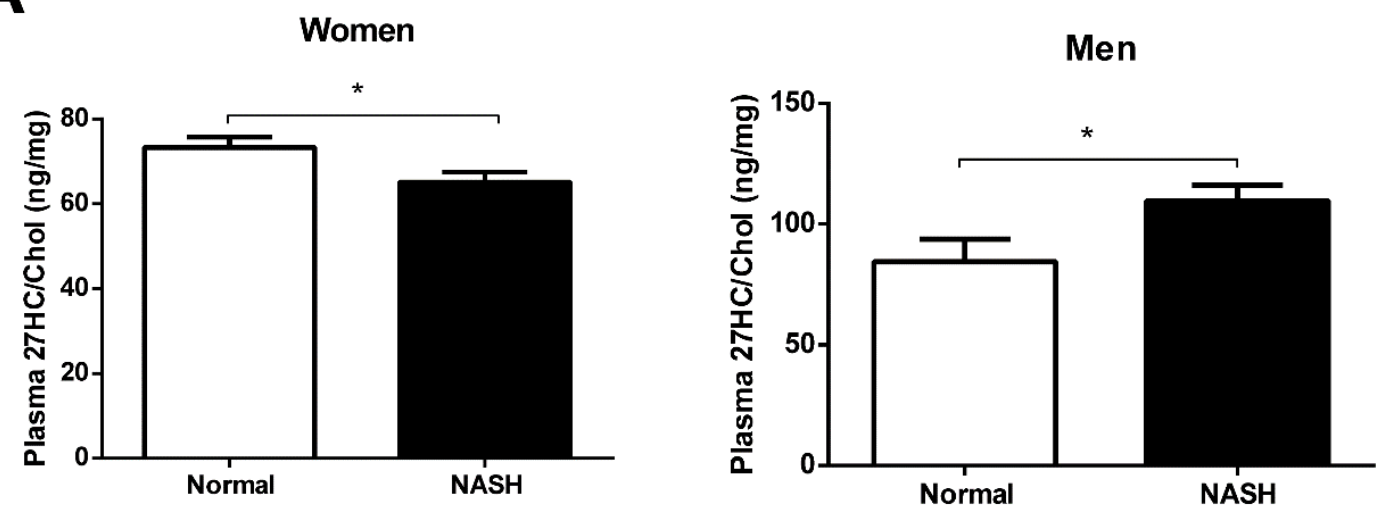

B
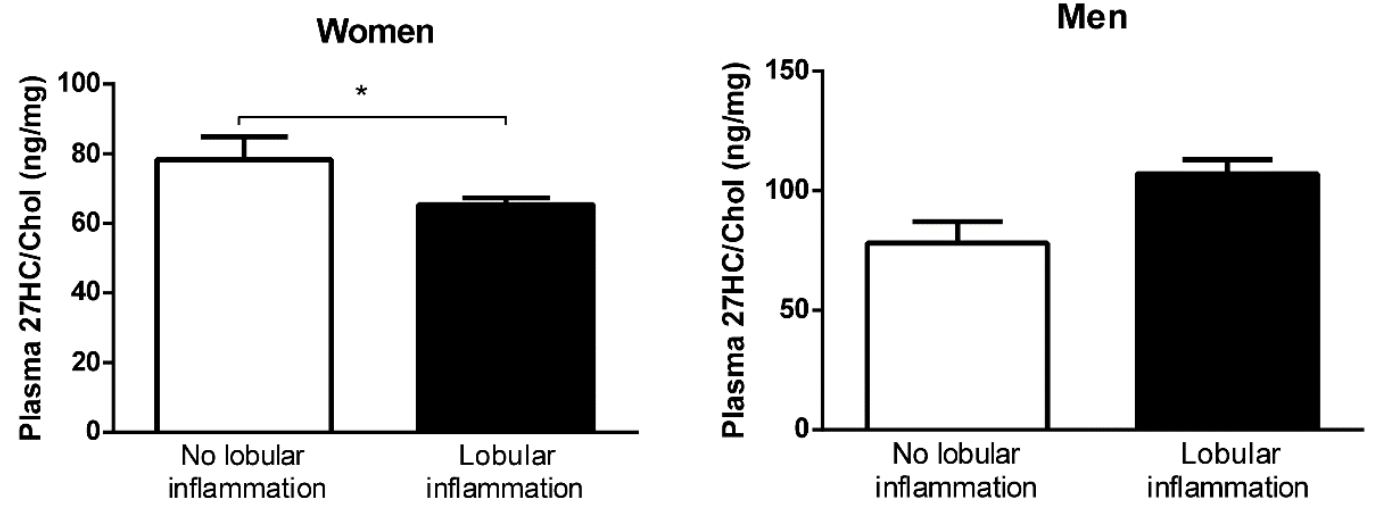

Figure 1: Relative plasma 27HC levels in female and male obese individuals in relation to hepatic inflammatory indicators. (A) Plasma $27 \mathrm{HC}$ levels in obese women and men categorized as 'NASH' or 'Normal' based on assessment of liver biopsies according to the criteria of Brunt. (B) Plasma 27HC levels in female and male obese individuals categorized according to presence of hepatic lobular inflammation. * Indicates $\mathrm{p} \leq 0.05$ compared to obese individuals categorized as 'Normal' (panel A) or obese individuals without hepatic lobular inflammation (panel B) by use of two-tailed unpaired $t$ test. Panel A and B show $n=44$ ( 21 female and 13 male individuals). All error bars are SEM. 


\section{Chapter 4}

Hepatic 27HC levels oppositely correlate with hepatic inflammation between female and male $N p c 1^{\text {nih }}$ mice

To validate the sex-opposed inflammatory effect of $27 \mathrm{HC}$ in vivo, hepatic inflammation and hepatic 27HC levels were assessed in seven week-old female and male Niemann-Pick type C1 disease (NPC1) mice (Npc1nih mice). Hepatic cryosections were stained for the inflammatory markers Mac-1 (infiltrated macrophages and neutrophils) and NIMP (neutrophils) and subsequently correlated with hepatic $27 \mathrm{HC}$ levels. Hepatic $27 \mathrm{HC}$ levels inversely correlated with both hepatic infiltrated macrophages and neutrophils $(r=-0.8605$; Table 1 and Supplementary figure S3A-B) and hepatic neutrophils ( $r=-0.9669$; Table 1 and Supplementary figure S3A-B) in female Npc1nih mice. In contrast, male Npc1nih mice showed a positive correlation between hepatic 27HC levels and hepatic infiltrated macrophages and neutrophils ( $\mathrm{r}=0.9595$; Table 1 and Supplementary figure S3A-B) and hepatic neutrophils $(r=0.88$; Table 1 and Supplementary figure S3A-B). Furthermore, no other significant differences were observed between female and male Npc1nih mice (Supplementary Table S4). As such, our data show that hepatic $27 \mathrm{HC}$ levels have sex-opposed hepatic inflammatory associations, validating Npc1nih mice as a model to investigate the sex-opposed inflammatory effects of $27 \mathrm{HC}$.

Table 1: Correlation analysis between hepatic 27-hydroxycholesterol levels and hepatic inflammation in seven weekold female and male $N p c 1^{\text {nih }}$ mice

\begin{tabular}{|c|c|c|c|}
\hline & \multicolumn{3}{|c|}{$\begin{array}{l}\text { Hepatic } 27 \mathrm{HC} / \text { Chol levels } \\
\text { (ng/mg) }\end{array}$} \\
\hline & Pearson $\mathbf{r}$ & $\mathbf{R}^{2}$ & $p$ value \\
\hline \multicolumn{4}{|l|}{ Female } \\
\hline $\begin{array}{l}\text { Hepatic infiltrated macrophages and neutrophils } \\
\text { (\# positive cells } / \mathrm{mm}^{2} \text { ) }\end{array}$ & -0.8605 & 0.7404 & 0.13 \\
\hline Hepatic neutrophils (\# positive cells $/ \mathrm{mm}^{2}$ ) & -0.9669 & 0.9349 & $0.03 *$ \\
\hline \multicolumn{4}{|l|}{ Male } \\
\hline $\begin{array}{l}\text { Hepatic infiltrated macrophages and neutrophils } \\
\text { (\# positive cells } / \mathrm{mm}^{2} \text { ) }\end{array}$ & 0.9595 & 0.9206 & $0.04^{*}$ \\
\hline Hepatic neutrophils (\# positive cells $/ \mathrm{mm}^{2}$ ) & 0.8861 & 0.7851 & 0.11 \\
\hline
\end{tabular}

*Indicates $p \leq 0.05$ by means of Pearson correlation. $n=4$ per experimental group 


\section{Chapter 4}

\section{Administration of $27 \mathrm{HC}$ in $\mathrm{Npc}^{\text {nih }}$ mice results in opposite hepatic inflammatory responses between sexes.}

To determine whether $27 \mathrm{HC}$ induces the sex-opposed effects on hepatic inflammation, female and male Npc1nih mice were given daily subcutaneous injections of $27 \mathrm{HC}$ or vehicle for 21 weeks. Hepatic and bile 27HC levels increased in both female and male Npc1nih mice upon 27HC administration, confirming successful administration of 27HC (Supplementary figure S4). In line with our hypothesis, administration of $27 \mathrm{HC}$ in female Npc1nih mice reduced hepatic inflammation, as observed by $\mathrm{HE}$ staining (Fig. 2A and Supplementary figure S5) and decreased numbers of infiltrated macrophages and neutrophils (Fig. 2B) in comparison to non-treated animals. In contrast, administration of 27HC in male Npc1nih mice resulted in a pro-inflammatory response compared to vehicle-treated male Npc1nih mice (Fig. 2A-B). These histological observations were further substantiated by hepatic gene expression levels for the inflammatory markers tumor necrosis factor alpha (TNFA), chemokine (C-X-C motif) ligand 2 (CXCL2), cluster of differentiation 68 (CD68), integrin alpha M (ITGAM), intercellular adhesion molecule 1 (ICAM) and interleukin 18 (IL18) (Fig. 2C), showing tendencies towards decreased or increased inflammation in female or male Npc1nih mice, respectively. Moreover, the interaction term provided by two-way ANOVA analysis was significant among all hepatic inflammatory analyses (Table 2), indicating that the hepatic inflammatory effect of $27 \mathrm{HC}$ is influenced by sex. 


\section{Chapter 4}

A

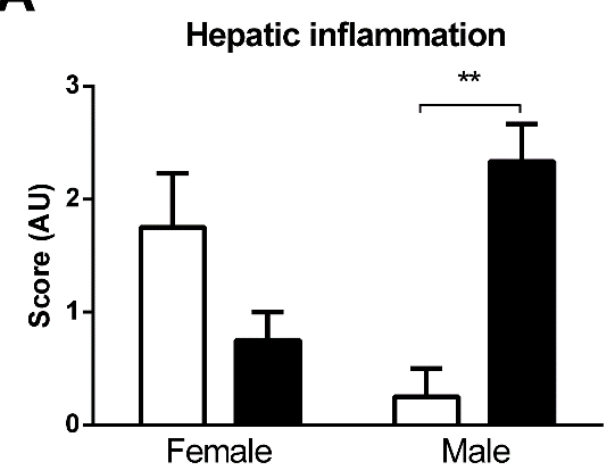

C

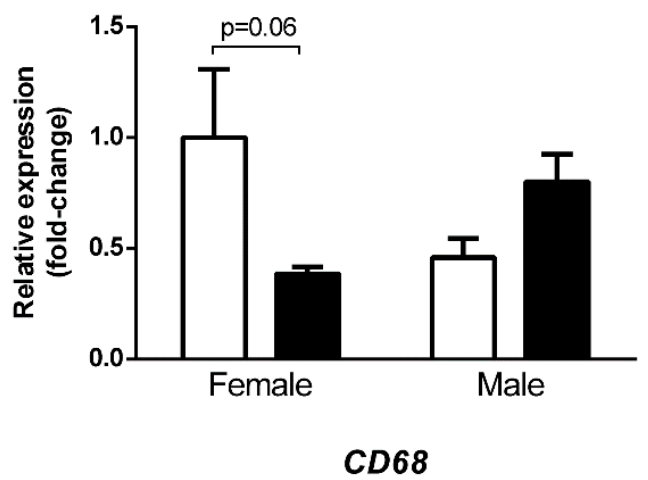

B

Infiltrated macrophages and neutrophils

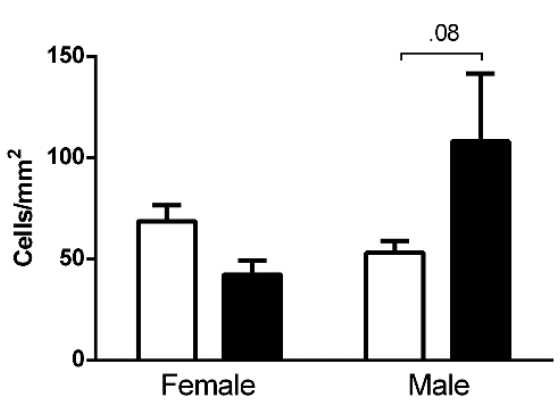

CXCL2

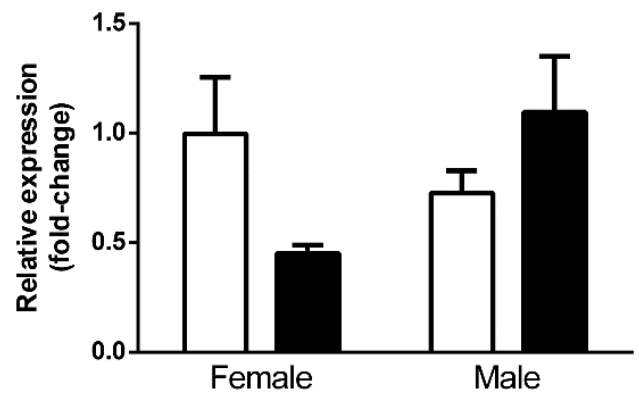

ITGAM

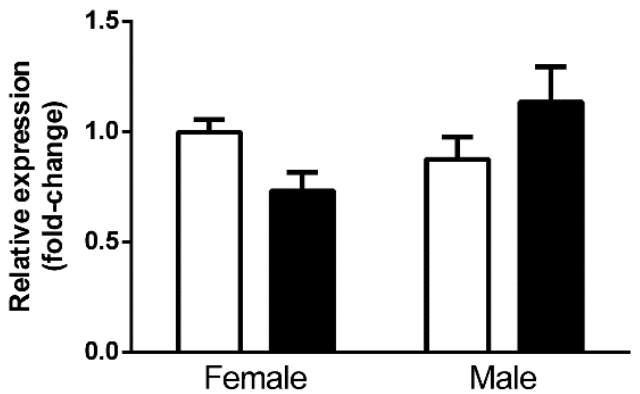

ICAM

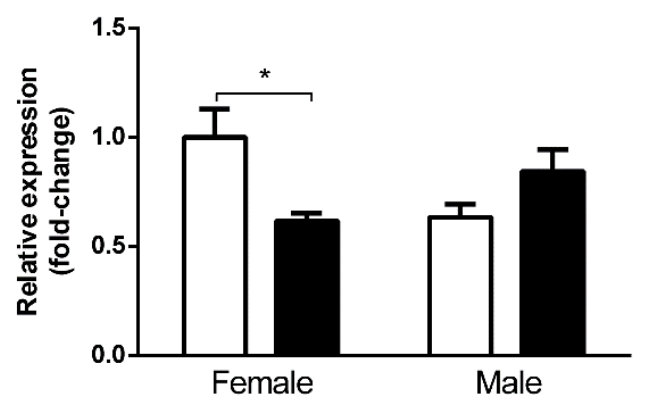

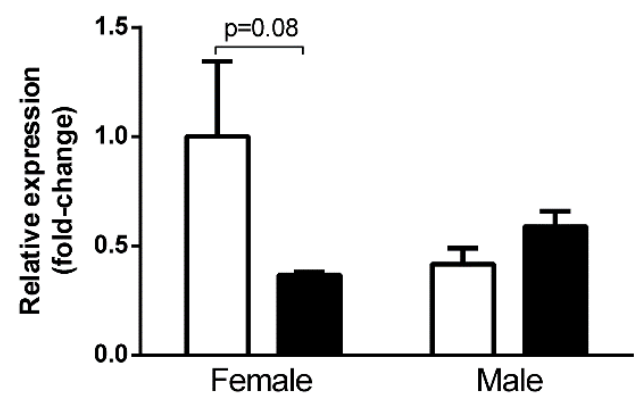

IL18

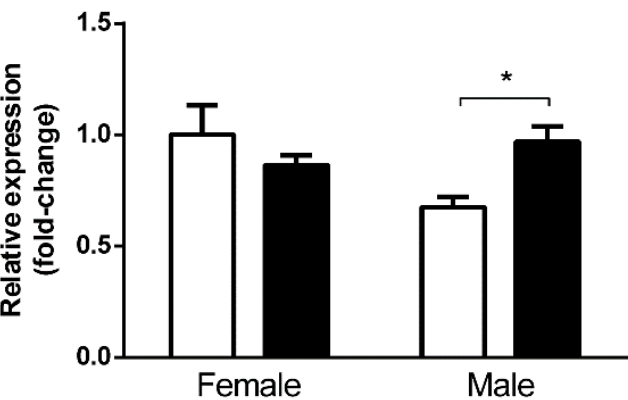

Figure 2: Hepatic inflammatory parameters of female and male Npc1nih mice after 27HC administration. (A-B) Quantification of haematoxylin and eosin (HE) staining and Mac-1 immunostaining of livers from female and male Npc1nih mice treated with or without 27HC. (C) Hepatic gene expression analysis of TNFA, CXCL2, CD68, ITGAM, ICAM and IL18 of female and male Npc1nih mice treated with or without $27 \mathrm{HC}$. ${ }^{*}$ Indicates $\mathrm{p} \leq 0.05$ and ${ }^{* *} \mathrm{p} \leq 0.01$ by use of two-way ANOVA with Tukey's post-hoc correction. $\mathrm{n}=4-5$ mice per experimental group. All error bars are SEM. 


\section{Chapter 4}

Table 2: Statistics of sex-opposed hepatic inflammatory effects of 27HC in Npc1nih mice

\begin{tabular}{l|c|} 
& $\begin{array}{c}\text { p value } \\
(\text { sex*27HC treatment interaction } \\
\text { term })\end{array}$ \\
\hline Histology & $0.001^{* *}$ \\
Hepatic inflammation (H\&E) & $0.0181^{*}$ \\
Infiltrated macrophages and & \\
neutrophils & $0.0351^{*}$ \\
\hline Gene Expression & $0.0066^{* *}$ \\
CD68 & $0.04^{*}$ \\
TNFA & $0.0142^{*}$ \\
CXCL2 & $0.0051^{* *}$ \\
IL18 & $0.0256^{*}$
\end{tabular} \mid

$\mathrm{n}=4-5$ mice per experimental group 


\section{Chapter 4}

Only in the presence of $\mathrm{E} 2$, the inflammatory effects of $27 \mathrm{HC}$ are sex-dependent in murine bone marrow-derived macrophages

As 27HC has been identified as a selective estrogen receptor modulator (SERM) [14] and estrogen is known to influence inflammatory processes [28], we first assessed the effect of $27 \mathrm{HC}$ in murine BMDMs cultured in E2-depleted and -enriched conditions by measuring nitric oxide (NO), a key mediator of inflammation. Vehicle-treated BMDMs cultured in E2-enriched medium showed reduced production of NO compared to vehicle-treated BMDMs cultured in E2-depleted ('Control') medium, confirming the modulatory role of E2 on NO production (Fig. 3A-B). Strikingly, treating BMDMs cultured in E2-enriched medium with $27 \mathrm{HC}$ resulted in an increased $\mathrm{NO}$ production, which was opposite in BMDMs cultured in E2-depleted medium, where treatment with $27 \mathrm{HC}$ reduced $\mathrm{NO}$ production (Fig. 3A-B). These results confirm that $27 \mathrm{HC}$ modifies the estrogen-mediated inhibition of NO production, and therefore inflammation.

Next, we investigated whether the sex-opposed inflammatory effects of 27HC are E2-dependent. For this purpose, Wt BMDMs derived from female or male mice were cultured in E2-depleted or E2enriched medium and treated with $27 \mathrm{HC}(1.0 \mu \mathrm{M}$ or $1.5 \mu \mathrm{M})$ or vehicle for 24 hours. Under E2-depleted conditions, 27HC-treatment reduced TNFa levels in female and male-derived BMDMs (Fig. 3B, left panel). In contrast, under E2-enriched conditions, 27HC-treatment only reduced TNFa levels in females, but not in males (Fig. 3B, right panel). Moreover, upon incubation with $1.5 \mu \mathrm{M}$ of $27 \mathrm{HC}$, TNFa levels were significantly increased in males (Fig. 3B, right panel). These observations were confirmed at gene expression level, showing similar trends for TNFA, ICAM and IL1B expression (Supplementary figure S6A). Sex, however, did not influence IL-10 levels after 27HC treatment (Supplementary figure S6B). Additionally, to confirm whether these inflammatory effects were more pronounced in an extreme model of cholesterol-induced inflammation, female- and male-derived Npc1nih BMDMs were exposed to similar conditions as Wt BMDMs. As expected, only under E2enriched conditions, the sex-opposed inflammatory effects of $27 \mathrm{HC}$ became apparent, demonstrating reduced TNFa levels in females and increased TNFa levels in males (Fig. 3C and Table 3). This observation was also further confirmed at gene expression level for TNFA and ICAM (Fig. 3D and Table 3). Together, these findings indicate that the sex-opposed inflammatory effects of 27HC are E2dependent and imply that the downstream E2-signaling is different in females and males. 
A

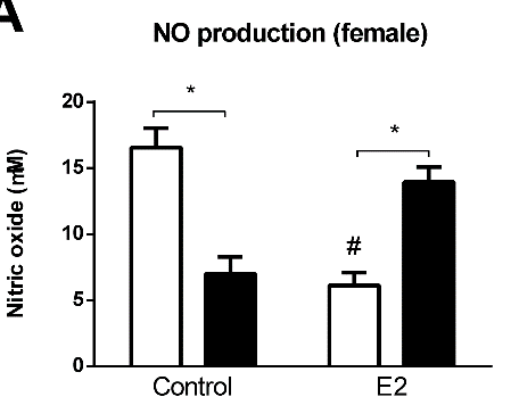

B

$$
\text { TNFa secretion }
$$
(E2-depleted conditions)

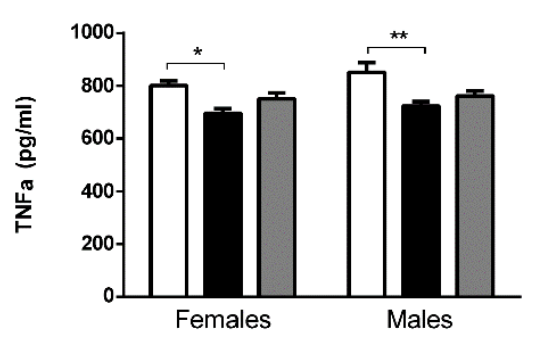

C
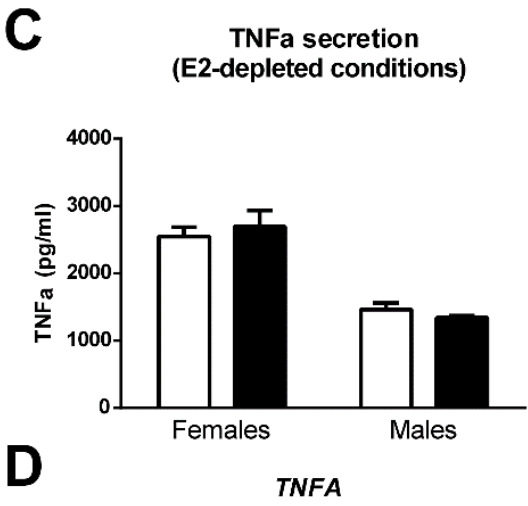

(E2-depleted conditions)

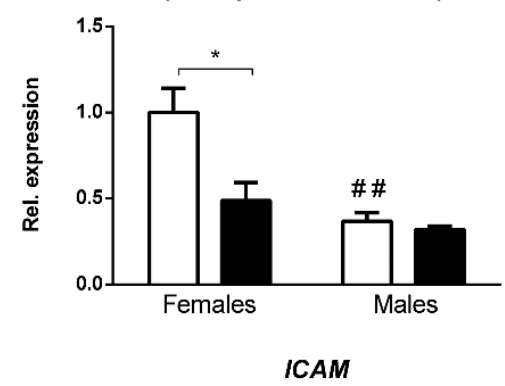

(E2-depleted conditions)

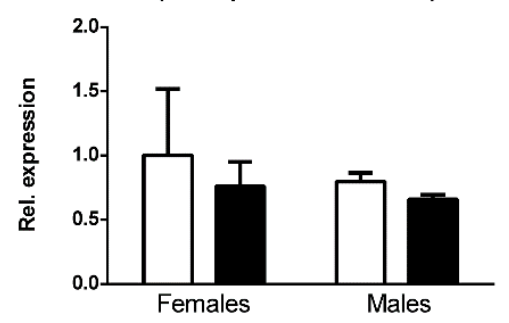

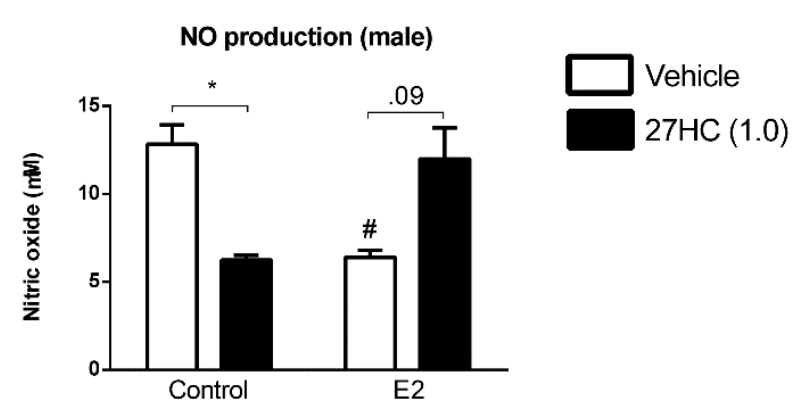

TNFa secretion (E2-enriched conditions)

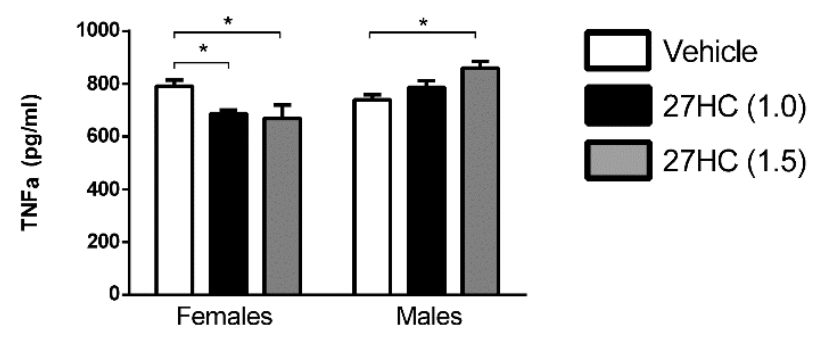

TNFa secretion

(E2-enriched conditions)

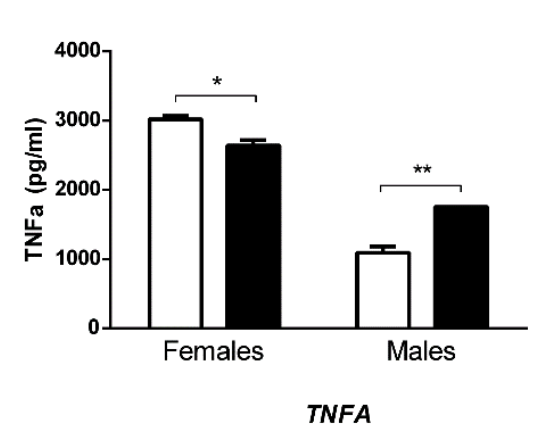

(E2-enriched conditions)

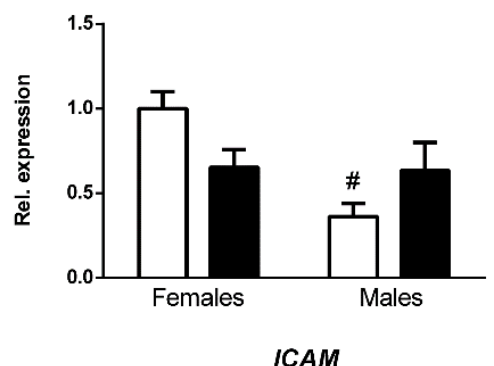

(E2-enriched conditions)

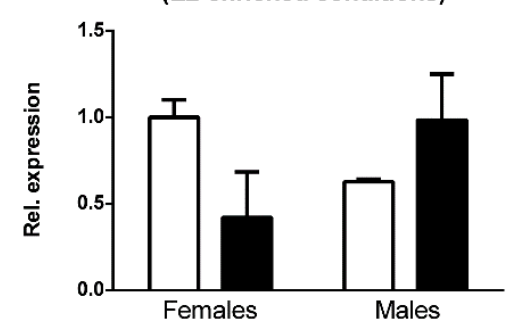

Figure 3: Inflammatory indicators of female and male-derived Wt bone marrow-derived macrophages cultured in E2-enriched/-depleted medium treated with 27HC. (A) Nitric oxide (NO) production in female (left panel) and male (right panel) wildtype BMDMs. * Indicates $\mathrm{p} \leq$ 


\section{Chapter 4}

0.05 compared to vehicle-treated BMDMs by use of two-way ANOVA with Tukey's post-hoc correction.\# Indicates $\mathrm{p} \leq 0.05$ compared to E2-depleted ('Control') BMDMs treated with vehicle. (B) TNF $\alpha$ protein levels in female and male Wt BMDMs. * Indicates $\mathrm{p} \leq 0.05$ and $* *$ $\mathrm{p} \leq 0.01$ compared to vehicle-treated female- or male-derived BMDMs cultured under E2-depleted conditions by use of two-way ANOVA with Tukey's post-hoc correction (left panel). $* \mathrm{p} \leq 0.05$ is compared to vehicle-treated female- or male-derived BMDMs cultured under E2enriched conditions (right panel). Results are shown as the average of 3 independent experiments. All error bars are SEM. (C) TNF $\alpha$ protein levels in female and male Npc1nih BMDMs. * Indicates $\mathrm{p} \leq 0.05$ and $* * \mathrm{p} \leq 0.01$ compared to vehicle-treated female- or male-derived BMDMs cultured under E2-enriched conditions by use of two-way ANOVA with Tukey's post-hoc correction (right panel). Results are shown as the average of 1 independent experiment. All error bars are SEM. (D) Gene expression analysis of TNFA and ICAM in female and male Npc1nih BMDMs. * Indicates $\mathrm{p} \leq 0.05$ compared to vehicle-treated female- or male-derived BMDMs cultured under E2-enriched conditions by use of two-way ANOVA with Tukey's post-hoc correction. \# $\mathrm{p} \leq 0.05$ and \# \# $\mathrm{p} \leq 0.01$ compared to vehicle-treated female BMDMs by use of two-way ANOVA with Tukey's post-hoc correction. Results are shown as the average of 1 independent experiment. All error bars are SEM.

Table 3: Statistics of sex-opposed inflammatory effects of 27HC in Npc1nih BMDMs

\begin{tabular}{r|c|} 
& $\begin{array}{c}\text { p value } \\
(\text { sex*27HC treatment } \\
\text { interaction term })\end{array}$ \\
\hline TNFa secretion (protein) & \\
E2-depleted & 0.3805 \\
E2-enriched & $0.0014 * *$ \\
Tnfa (gene expression) & \\
E2-depleted & $0.0360 *$ \\
E2-enriched & $0.0468^{*}$ \\
E2-depleted & \\
E2-enriched & 0.8107
\end{tabular}

*Indicates $p \leq 0.05$ and ${ }^{* *} p \leq 0.01$ by two-way ANOVA. $\mathrm{n}=3$ per experimental group 


\section{Chapter 4}

\section{Female BMDMs show higher ER $\alpha$ expression compared to male BMDMs}

To provide insight into the underlying mechanism that explains the E2-driven opposite inflammatory effect of $27 \mathrm{HC}$ on females and males, we determined protein levels of estrogen receptors alpha and beta $(E R \alpha$ and $E R \beta)$ and liver $X$ receptor beta $(L X R \beta)$ in untreated Wt BMDMs derived from female and male mice (Fig. 4A-B). While ER $\beta$ and LXR $\beta$ levels were similar between females and males, ER $\alpha$ levels showed a near three-fold higher protein expression in female compared to male BMDMs (Fig 4A-B). Moreover, in females, ER $\alpha$ expression was two-fold higher than ER $\beta$ expression, while in males ER $\alpha$ and ER $\beta$ show near equal protein expression (Fig. 4A). Considering the modulating effect of these estrogen receptor subtypes on inflammatory responses, our findings suggest that the sex-opposed inflammatory effect of $27 \mathrm{HC}$ might be related to different ER $\alpha$ expression between female and male macrophages.

A

ERa and ERb expression

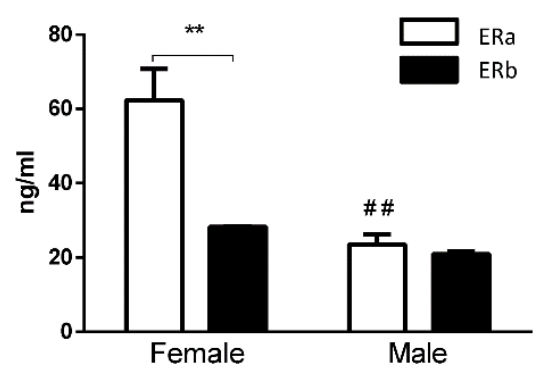

B

LXRb protein expression

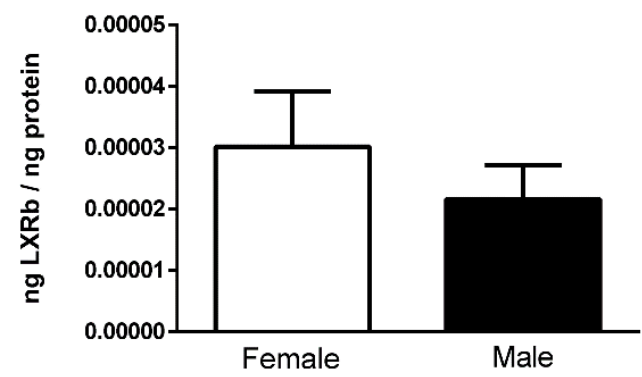

Figure 4 Protein expression of ER $\alpha, \operatorname{ER} \beta$ and LXR $\beta$ in female and male Wt bone marrow-derived macrophages. (A) ER $\alpha$ and ER $\beta$ and (B) LXR $\beta$ expression in female and male BMDMs. ** Indicates $\mathrm{p} \leq 0.01$ compared to female ER $\beta$ expression and \# \# indicates $\mathrm{p} \leq 0.01$ compared to female ER $\alpha$ expression by use of two-way ANOVA with Tukey's post-hoc correction. Results are shown as the average of 3 independent experiments. All error bars are SEM. 


\section{Chapter 4}

\section{Discussion}

Despite the increased awareness of differences in the inflammatory response between men and women, only limited research has focused on the biological factors underlying these sex-opposed effects. Here, we identify $27 \mathrm{HC}$ as a biological factor exerting sex-opposed effects on inflammation and show that these sex-opposed effects are mediated via differences in estrogen signaling. Hence, the individual's sex needs to be taken into account when $27 \mathrm{HC}$ is employed as therapeutic tool as well as in macrophage estrogen research in general.

An increasing amount of research is pointing towards the therapeutic potential of bile acids (and their precursors) to modulate the inflammatory response in metabolic diseases such as cardiovascular diseases [29], diabetes [30], NASH [31] and even in pulmonary diseases [32,33]. With regard to this evolving trend, we demonstrate in the current study that sex plays a key role on the inflammatory effect of the bile acid precursor $27 \mathrm{HC}$, which likely explains the previous contradictive results in atherosclerosis [22] and NASH [20,21]. Our findings suggest that bile acids and their precursors potentially have opposite inflammatory effects in females and males, warranting caution on the use of bile acids as therapeutical tool in the clinic without conducting sex-specific clinical trials. Indeed, in the past, sex bias has been identified in the search for new therapeutic drugs in both preclinical animal studies as well as in clinical human studies that included more male than female individuals $[34,35]$. In the cardiovascular field, this phenomenon has led to differences in the clinical outcome of males vs. females in relation to key drugs such as beta-blockers, angiotensin-converting enzyme inhibitors, diuretics and anti-arrhythmics [35]. As, to our knowledge, this is the first study to describe sex-related differences in the context of bile acids, future research is necessary to validate our observation. So far, gender-specific health care is only present in very limited quantities and epidemiological research proving sex differences [36,37] are only recently finding their way to larger scientific audiences, which is exemplified by funding agencies specifically endorsing research investigating female/male differences $[38,39]$. Our findings therefore add fuel to the increasing concept of men and women as two different entities whose health and disease condition should be approached from a different perspective.

While a chronic low grade inflammatory state is as a key factor contributing to the prevalence of obesity-associated disorders $[6,7,40]$ only recently it has become clear that there are differences between the sexes concerning this obesity-associated inflammatory response. Plasma adiponectin concentrations were demonstrated to decrease more dramatically in obese male than female patients [41], providing evidence for a more detrimental inflammatory state in obese male compared obese female patients. Additionally, whereas male high-fat diet-induced obese mice showed profound adipose tissue macrophage accumulation [42] and infiltration [43], female obese mice were protected from this 


\section{Chapter 4}

adipose tissue inflammatory response $[42,43]$. Singer et al. suggested that the absence of adipose tissue inflammation in female high-fat diet-induced obese mice is likely due to the formation of CD11c- (M2like) macrophages, while matched male mice show expansion of CD11c+ (M1-like) macrophages in adipose tissue [44]. These findings suggest a key role for macrophages in mediating opposite inflammatory responses between females and males. In line with this view, we here observed that the cholesterol derivative $27 \mathrm{HC}$ exerts anti-inflammatory effects in livers of females and pro-inflammatory effects in livers of males and we could replicate these findings in primary macrophages as well as validate them in a cohort of obese individuals. The sex-dependent inflammatory influence of $27 \mathrm{HC}$ on macrophages has been confirmed in other experiments showing that $27 \mathrm{HC}$ exerts pro-inflammatory effects on male-derived macrophages [45], whereas anti-inflammatory effects are observed in femalederived macrophages [20]. Therefore, future studies focusing on the inflammatory effect of $27 \mathrm{HC}$ in macrophages have to consider sex as a potential variable influencing the outcome of their studies.

The observation that the presence of estrogen is essential to observe the sex-opposed inflammatory effects of $27 \mathrm{HC}$ in BMDMs indicates a key role for estrogen in the 27HC-induced effect on inflammation. This observation is in line with previous findings of Umetani et al. who linked 27HC to estrogen by identifying $27 \mathrm{HC}$ as the first endogenous selective estrogen receptor modulator (SERM) [14]. Estrogens are known to dampen, reduce and inhibit inflammatory responses by various mechanisms, including via activation of ER dimers that repress transcription of, for instance, iNOS [46]. Indeed, we found that estrogen reduces NO production and, additionally, that administration of $27 \mathrm{HC}$ in the absence of $\mathrm{E} 2$ resulted in a similar decrease in NO production, suggesting a similar effect of $\mathrm{E} 2$ and $27 \mathrm{HC}$ on ER dimers. Simultaneous administration of E2 and 27HC on the other hand causes a conformational change in ER dimers [46], blocking their repressive effect on transcription and explaining the increased NO production. These observations therefore confirm that $27 \mathrm{HC}$ is a SERM. Additionally, besides ER dimers, estrogens also influence inflammation via repressing the transcription factor NF-қB [28], a master regulator of inflammation [47]. Indeed, we found that downstream targets of NF-қB, TNF $\alpha$, ICAM and IL1b, decreased in female-derived, but not in male-derived, BMDMs cultured in E2-enriched medium that were treated with $27 \mathrm{HC}$. As production of the inflammatory mediator NO (which is not NF-қВ-mediated) [48] was not differentially influenced by $27 \mathrm{HC}$ among the sexes, our observations provide evidence that the sex-opposed inflammatory effect of $27 \mathrm{HC}$ is likely related to NF-қB-mediated mechanisms.

Additionally, these findings further suggest that the sex-opposed inflammatory effects of $27 \mathrm{HC}$ are at least partly mediated via differences in downstream estrogen signaling of macrophages. This observation raises the question how downstream estrogen signaling in macrophages is different in females and males. While estrogen exerts anti-inflammatory effects in both females and males [49], a 


\section{Chapter 4}

potential answer to this exciting question might be related to sex differences in the distribution of the subtypes of the ER, ER alpha (ER $\alpha$ ) and ER beta (ERß). Indeed, while ER $\beta$ expression was similar, we observed a substantial increased expression of ER $\alpha$ in female compared to male BMDMs, suggesting females show more pronounced ER $\alpha$-mediated macrophage responses that underlie the observed antiinflammatory effect of $27 \mathrm{HC}$ in females. In line with this observation, ER $\alpha$ expression in macrophages has been linked to anti-inflammatory responses in skin repair [50] and infectious immunity [51]. Moreover, while ER $\alpha$ and ER $\beta$ share a high degree of homology, their target genes differ substantially indicating that the binding of ligands (such as $27 \mathrm{HC}$ ) to different ER subtypes impacts the fate of the inflammatory signaling [52]. For these reasons, future research should further investigate whether differences in the distribution of ER $\alpha$ and $\mathrm{ER} \beta$ determine the inflammatory fate of $27 \mathrm{HC}$. The different $\mathrm{ER} \alpha$ expression levels between females and males also highlight sex as an essential factor to be considered when investigating macrophage estrogen signaling, irrespective of $27 \mathrm{HC}$ levels. As such, for future research, it is indispensable to determine whether the macrophages under investigation are derived from female or male sources.

As cholesterol levels increase during obesity in men and women [53] and 27HC levels are tightly correlated with cholesterol levels, our findings suggest that increased cholesterol levels will result in a more detrimental inflammatory response in males compared to females. Indeed, previous reports have described that premenopausal women are better protected from metaflammatory insults compared to male individuals [54]. Additionally, the premenopausal inflammatory resilience in women reverses after menopause [55], suggesting that estrogen plays a key role in mediating the metaflammatory response. Nevertheless, estrogen administration in postmenopausal women resulted in a paradoxical increased risk of inflammation and cardiovascular disease [56,57], suggesting that other biological factors might be involved in the inflammatory response in females. Considering our findings that $27 \mathrm{HC}$ is affecting inflammation via perturbing estrogen signaling and the previous observation of increased LDL cholesterol (and concomitantly 27HC) levels after menopause [58], an interesting line for future research is to explore whether the inflammatory effect of $27 \mathrm{HC}$ is different in pre- versus postmenopausal women.

Overall, we here show that $27 \mathrm{HC}$ exerts sex-opposed effects on cholesterol-induced inflammatory responses that are potentially mediated via sex-related expression differences of ER $\alpha$. Future studies should investigate how these findings have implications for gender-based therapeutic strategies against inflammation. 


\section{Chapter 4}

\section{References}

1. Villapol S, Loane DJ, Burns MP. Sexual dimorphism in the inflammatory response to traumatic brain injury. Glia 2017; 65: 1423-1438.

2. Kay E, Gomez-Garcia L, Woodfin A, et al. Sexual dimorphisms in leukocyte trafficking in a mouse peritonitis model. J Leukoc Biol 2015; 98: 805-817.

3. Griffin C, Lanzetta N, Eter L, et al. Sexually dimorphic myeloid inflammatory and metabolic responses to diet-induced obesity. Am J Physiol Regul Integr Comp Physiol 2016; 311: R211216.

4. Fulham MA, Mandrekar P. Sexual Dimorphism in Alcohol Induced Adipose Inflammation Relates to Liver Injury. PLoS One 2016; 11: e0164225.

5. Regitz-Zagrosek V. Sex and gender differences in health. Science \& Society Series on Sex and Science. EMBO Rep 2012; 13: 596-603.

6. Weisberg SP, McCann D, Desai M, et al. Obesity is associated with macrophage accumulation in adipose tissue. J Clin Invest 2003; 112: 1796-1808.

7. $\mathrm{Xu} \mathrm{H}$, Barnes GT, Yang Q, et al. Chronic inflammation in fat plays a crucial role in the development of obesity-related insulin resistance. J Clin Invest 2003; 112: 1821-1830.

8. Hotamisligil GS. Inflammation, metaflammation and immunometabolic disorders. Nature 2017; 542: 177-185.

9. Younossi Z, Anstee QM, Marietti M, et al. Global burden of NAFLD and NASH: trends, predictions, risk factors and prevention. Nat Rev Gastroenterol Hepatol 2018; 15: 11-20.

10. Wouters K, van Gorp PJ, Bieghs V, et al. Dietary cholesterol, rather than liver steatosis, leads to hepatic inflammation in hyperlipidemic mouse models of nonalcoholic steatohepatitis. Hepatology 2008; 48: 474-486.

11. Guillemot-Legris O, Mutemberezi V, Muccioli GG. Oxysterols in Metabolic Syndrome: From Bystander Molecules to Bioactive Lipids. Trends Mol Med 2016; 22: 594-614.

12. Ertunc ME, Hotamisligil GS. Lipid signaling and lipotoxicity in metaflammation: indications for metabolic disease pathogenesis and treatment. J Lipid Res 2016; 57: 2099-2114.

13. Brown AJ, Jessup W. Oxysterols and atherosclerosis. Atherosclerosis 1999; 142: 1-28. 


\section{Chapter 4}

14. Umetani M, Domoto H, Gormley AK, et al. 27-Hydroxycholesterol is an endogenous SERM that inhibits the cardiovascular effects of estrogen. Nat Med 2007; 13: 1185-1192.

15. Tontonoz P, Mangelsdorf DJ. Liver X receptor signaling pathways in cardiovascular disease. Mol Endocrinol 2003; 17: 985-993.

16. Hendrikx T, Walenbergh SM, Hofker MH, et al. Lysosomal cholesterol accumulation: driver on the road to inflammation during atherosclerosis and non-alcoholic steatohepatitis. Obes Rev 2014; 15: 424-433.

17. Weber K, Schilling JD. Lysosomes integrate metabolic-inflammatory cross-talk in primary macrophage inflammasome activation. J Biol Chem 2014; 289: 9158-9171.

18. Ioannou GN. The Role of Cholesterol in the Pathogenesis of NASH. Trends Endocrinol Metab 2016; 27: 84-95.

19. Bieghs V, van Gorp PJ, Walenbergh SM, et al. Specific immunization strategies against oxidized low-density lipoprotein: a novel way to reduce nonalcoholic steatohepatitis in mice. Hepatology 2012; 56: 894-903.

20. Hendrikx T, Jeurissen ML, Bieghs V, et al. Hematopoietic overexpression of Cyp27a1 reduces hepatic inflammation independently of 27 -hydroxycholesterol levels in $\operatorname{Ldlr}(-/-)$ mice. J Hepatol 2015; 62: 430-436.

21. Bieghs V, Hendrikx T, van Gorp PJ, et al. The cholesterol derivative 27-hydroxycholesterol reduces steatohepatitis in mice. Gastroenterology 2013; 144: 167-178 e161.

22. Umetani M, Ghosh $\mathrm{P}$, Ishikawa $\mathrm{T}$, et al. The cholesterol metabolite 27-hydroxycholesterol promotes atherosclerosis via proinflammatory processes mediated by estrogen receptor alpha. Cell Metab 2014; 20: 172-182.

23. Ramirez CM, Liu B, Taylor AM, et al. Weekly cyclodextrin administration normalizes cholesterol metabolism in nearly every organ of the Niemann-Pick type $\mathrm{C} 1$ mouse and markedly prolongs life. Pediatr Res 2010; 68: 309-315.

24. Verdam FJ, Dallinga JW, Driessen A, et al. Non-alcoholic steatohepatitis: a non-invasive diagnosis by analysis of exhaled breath. J Hepatol 2013; 58: 543-548.

25. Rensen SS, Slaats Y, Driessen A, et al. Activation of the complement system in human nonalcoholic fatty liver disease. Hepatology 2009; 50: 1809-1817.

26. Bieghs V, Verheyen F, van Gorp PJ, et al. Internalization of modified lipids by CD36 and SRA leads to hepatic inflammation and lysosomal cholesterol storage in Kupffer cells. PLoS One 2012; 7: e34378. 


\section{Chapter 4}

27. Bieghs V, Wouters K, van Gorp PJ, et al. Role of scavenger receptor A and CD36 in dietinduced nonalcoholic steatohepatitis in hyperlipidemic mice. Gastroenterology 2010; 138: 2477-2486, 2486 e2471-2473.

28. Straub RH. The complex role of estrogens in inflammation. Endocr Rev 2007; 28: 521-574.

29. Porez G, Prawitt J, Gross B, et al. Bile acid receptors as targets for the treatment of dyslipidemia and cardiovascular disease. J Lipid Res 2012; 53: 1723-1737.

30. Shapiro H, Kolodziejczyk AA, Halstuch D, et al. Bile acids in glucose metabolism in health and disease. J Exp Med 2018; 215: 383-396.

31. Neuschwander-Tetri BA, Loomba R, Sanyal AJ, et al. Farnesoid X nuclear receptor ligand obeticholic acid for non-cirrhotic, non-alcoholic steatohepatitis (FLINT): a multicentre, randomised, placebo-controlled trial. Lancet 2015; 385: 956-965.

32. Comeglio P, Filippi S, Sarchielli E, et al. Anti-fibrotic effects of chronic treatment with the selective FXR agonist obeticholic acid in the bleomycin-induced rat model of pulmonary fibrosis. J Steroid Biochem Mol Biol 2017; 168: 26-37.

33. Comeglio P, Morelli A, Adorini L, et al. Beneficial effects of bile acid receptor agonists in pulmonary disease models. Expert Opin Investig Drugs 2017; 26: 1215-1228.

34. Beery AK, Zucker I. Sex bias in neuroscience and biomedical research. Neurosci Biobehav Rev 2011; 35: 565-572.

35. Regitz-Zagrosek V. Therapeutic implications of the gender-specific aspects of cardiovascular disease. Nat Rev Drug Discov 2006; 5: 425-438.

36. Riecher-Rossler A. Sex and gender differences in mental disorders. Lancet Psychiatry 2017; 4: 8-9.

37. Mosca L, Barrett-Connor E, Wenger NK. Sex/gender differences in cardiovascular disease prevention: what a difference a decade makes. Circulation 2011; 124: 2145-2154.

38. Gohar A, Kievit RF, Valstar GB, et al. Opportunistic screening models for high-risk men and women to detect diastolic dysfunction and heart failure with preserved ejection fraction in the community. Eur J Prev Cardiol 2018: 2047487318816774.

39. Oren-Suissa M, Bayer EA, Hobert O. Sex-specific pruning of neuronal synapses in Caenorhabditis elegans. Nature 2016; 533: 206-211.

40. Lumeng CN, Saltiel AR. Inflammatory links between obesity and metabolic disease. J Clin Invest 2011; 121: 2111-2117. 


\section{Chapter 4}

41. Arita Y, Kihara S, Ouchi N, et al. Paradoxical decrease of an adipose-specific protein, adiponectin, in obesity. Biochem Biophys Res Commun 1999; 257: 79-83.

42. Varghese M, Griffin C, McKernan K, et al. Sex differences in inflammatory responses to adipose tissue lipolysis in diet-induced obesity. Endocrinology 2018.

43. Nickelson KJ, Stromsdorfer KL, Pickering RT, et al. A comparison of inflammatory and oxidative stress markers in adipose tissue from weight-matched obese male and female mice. Exp Diabetes Res 2012; 2012: 859395.

44. Singer K, Maley N, Mergian T, et al. Differences in Hematopoietic Stem Cells Contribute to Sexually Dimorphic Inflammatory Responses to High Fat Diet-induced Obesity. J Biol Chem 2015; 290: 13250-13262.

45. Kim BY, Son Y, Eo SK, et al. Diclofenac inhibits 27-hydroxycholesterol-induced inflammation. Biochem Biophys Res Commun 2016; 478: 1456-1461.

46. Khan D, Ansar Ahmed S. The Immune System Is a Natural Target for Estrogen Action: Opposing Effects of Estrogen in Two Prototypical Autoimmune Diseases. Front Immunol 2015; 6: 635.

47. Liu T, Zhang L, Joo D, et al. NF-kappaB signaling in inflammation. Signal Transduct Target Ther $2017 ; 2$.

48. Tripathi $\mathrm{P}$, Tripathi $\mathrm{P}$, Kashyap L, et al. The role of nitric oxide in inflammatory reactions. FEMS Immunol Med Microbiol 2007; 51: 443-452.

49. Villa A, Rizzi N, Vegeto E, et al. Estrogen accelerates the resolution of inflammation in macrophagic cells. Sci Rep 2015; 5: 15224.

50. Campbell L, Emmerson E, Williams H, et al. Estrogen receptor-alpha promotes alternative macrophage activation during cutaneous repair. J Invest Dermatol 2014; 134: 2447-2457.

51. Lambert KC, Curran EM, Judy BM, et al. Estrogen receptor-alpha deficiency promotes increased TNF-alpha secretion and bacterial killing by murine macrophages in response to microbial stimuli in vitro. J Leukoc Biol 2004; 75: 1166-1172.

52. Bolego C, Cignarella A, Staels B, et al. Macrophage function and polarization in cardiovascular disease: a role of estrogen signaling? Arterioscler Thromb Vasc Biol 2013; 33: 1127-1134.

53. Klop B, Elte JW, Cabezas MC. Dyslipidemia in obesity: mechanisms and potential targets. Nutrients 2013; 5: 1218-1240. 


\section{Chapter 4}

54. Williams JK, Adams MR, Klopfenstein HS. Estrogen modulates responses of atherosclerotic coronary arteries. Circulation 1990; 81: 1680-1687.

55. Cushman M, Legault C, Barrett-Connor E, et al. Effect of postmenopausal hormones on inflammation-sensitive proteins: the Postmenopausal Estrogen/Progestin Interventions (PEPI) Study. Circulation 1999; 100: 717-722.

56. Hulley S, Grady D, Bush T, et al. Randomized trial of estrogen plus progestin for secondary prevention of coronary heart disease in postmenopausal women. Heart and Estrogen/progestin Replacement Study (HERS) Research Group. JAMA 1998; 280: 605-613.

57. Pradhan AD, Manson JE, Rossouw JE, et al. Inflammatory biomarkers, hormone replacement therapy, and incident coronary heart disease: prospective analysis from the Women's Health Initiative observational study. JAMA 2002; 288: 980-987.

58. Stevenson JC, Crook D, Godsland IF. Influence of age and menopause on serum lipids and lipoproteins in healthy women. Atherosclerosis 1993; 98: 83-90. 


\section{Supplementary Figures}

\section{Supplementary figure S1}

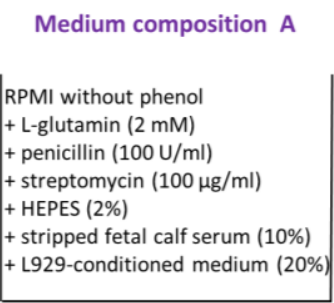

Medium composition B

RPMI without phenol + L-glutamin (2 mM)

+ penicillin $(100 \mathrm{U} / \mathrm{ml})$

+ streptomycin $(100 \mu \mathrm{g} / \mathrm{ml})$

+ HEPES $(2 \%)$

+ stripped fetal calf serum (10\%)

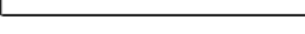

Medium composition $\mathrm{C}$

RPMI without phenol

+ L-glutamin (2 mM)

+ penicillin $(100 \mathrm{U} / \mathrm{ml})$

+ streptomycin $(100 \mu \mathrm{g} / \mathrm{ml})$

+ HEPES (2\%)

+ stripped fetal calf serum (10\%)

$+27 \mathrm{HC}(1 \mu \mathrm{M})$ or vehicle $(0.3 \%)$

$+\mathrm{E} 2(0.5 \mathrm{nM})$ or EtOH
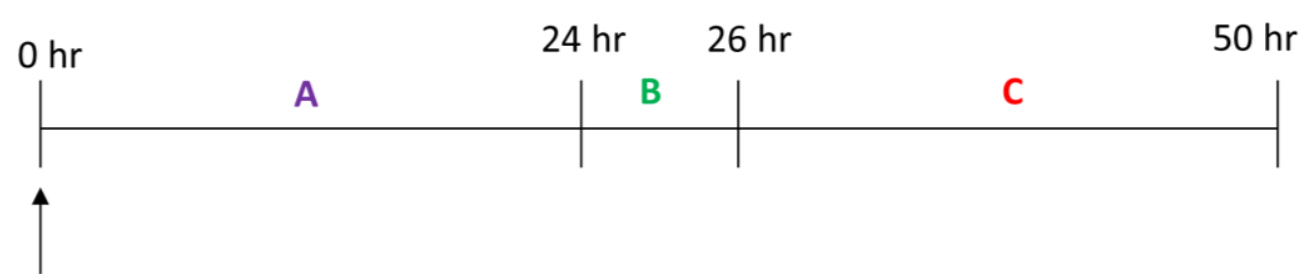

D9

Supplemental Figure 1: In vitro experimental set-up. After 9 days culture in regular RPMI medium (see Materials and methods), BMDMs were incubated in medium A to get rid of estrogen in the RPMI medium and FCS. Next, BMDMs were cultured for 2 hours without LCM medium (medium B) followed by 24 or $48 \mathrm{hr}$ incubation with 27HC $(1 \mu \mathrm{M})$ or vehicle (2-hydroxypropyl- $\beta$-cyclodextrin; $0.3 \%)$ in combination with E2 (0.5 nM) or EtOH. HEPES, (4-(2-hydroxyethyl)-1-piperazineethanesulfonic acid); 27HC, 27-hydroxycholesterol; E2, 17ß-estradiol, EtOH, ethanol 


\section{Chapter 4}

A

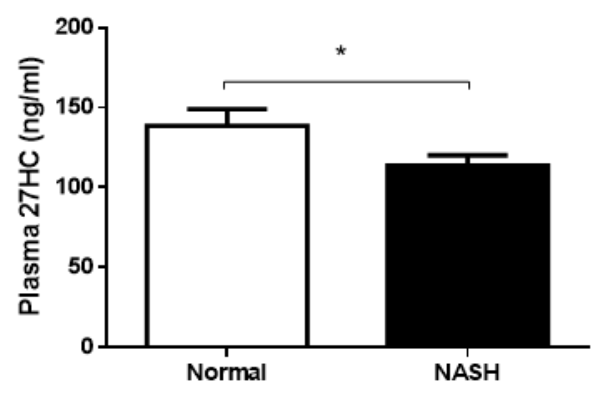

B

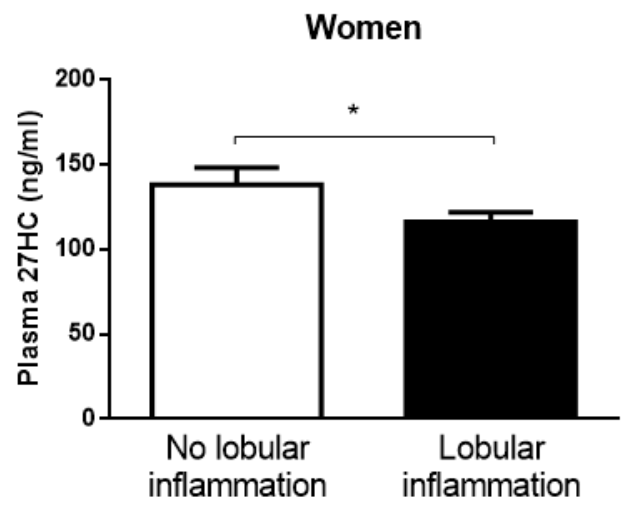

Men

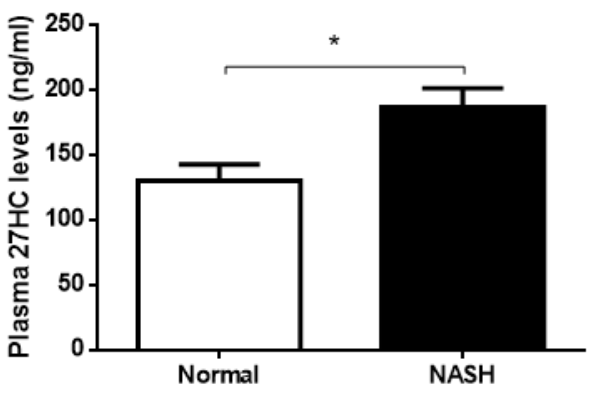

Men

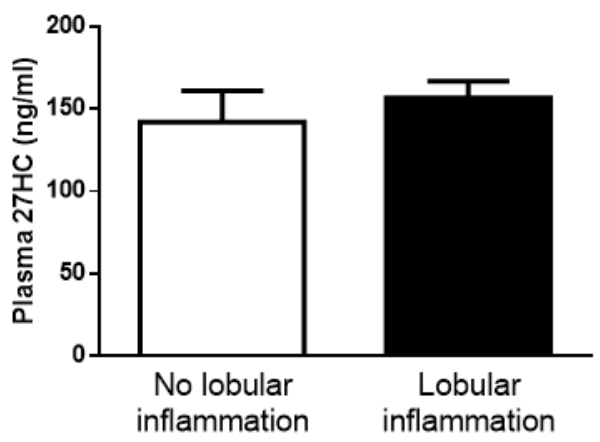

Supplemental Figure 2: Plasma absolute 27HC levels in female and male obese individuals in relation to hepatic inflammatory indicators. (A) Plasma 27HC levels in obese women and men categorized as 'NASH' or 'Normal' based on assessment of liver biopsies according to the criteria of Brunt. (B) Plasma $27 \mathrm{HC}$ levels in female and male obese individuals categorized according to presence of hepatic lobular inflammation. * Indicates $\mathrm{p} \leq 0.05$ compared to obese individuals categorized as 'Normal' (panel A) or obese individuals without hepatic lobular inflammation (panel B) by use of two-tailed unpaired $t$ test. Panel A and B show $n=44$ (21 female and 13 male individuals). All error bars are SEM. 


\section{Chapter 4}
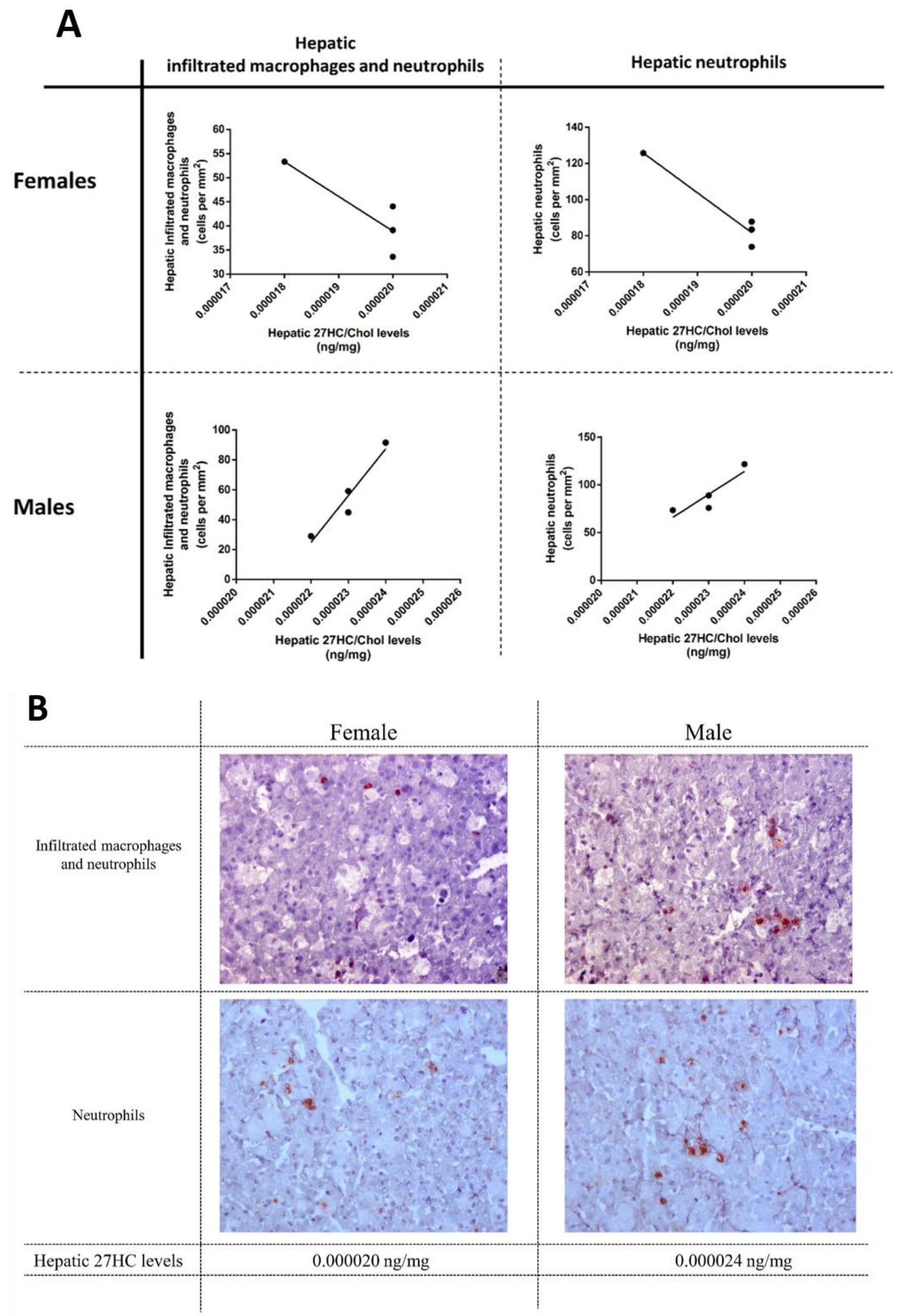

Supplemental Figure 3: Hepatic inflammation in female and male Npc1nih mice. (A) Correlation analysis of hepatic 27HC levels with immunostaining quantification for hepatic infiltrated macrophages and neutrophils (Mac-1) and hepatic neutrophils (NIMP staining) in seven week-old female and male Npc1nih mice. $n=4$ mice per experimental group. (B) Representative images (200x magnification) of a female and male Npc1nih mouse with respective hepatic $27 \mathrm{HC}$ levels. 


\section{Chapter 4}
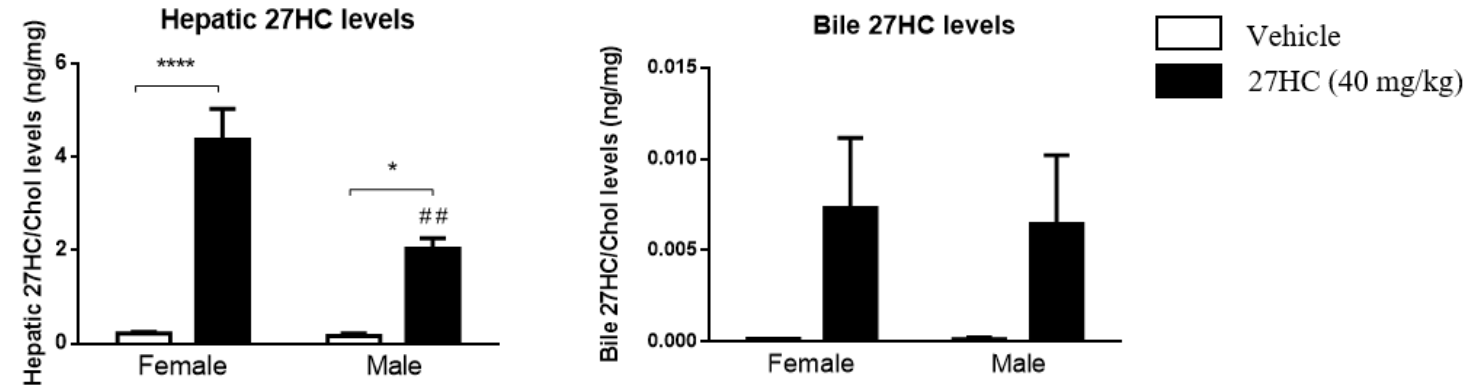

Supplemental Figure 4: Bile acids in female and male Npc1nih mice. Hepatic and bile 27HC levels in 27HC-administered Npc1nih mice. $\mathrm{n}=4-5$ mice per experimental group. * Indicates $\mathrm{p} \leq 0.05$ and $* * * * \mathrm{p} \leq 0.0001$ compared to vehicle-treated female or male Npc1nih mice by use of two-way ANOVA with Tukey's post-hoc correction.\# \# Indicates $p \leq 0.01$ compared to 27HC-treated female Npc1nih mice. All error bars are SEM. 


\section{Chapter 4}

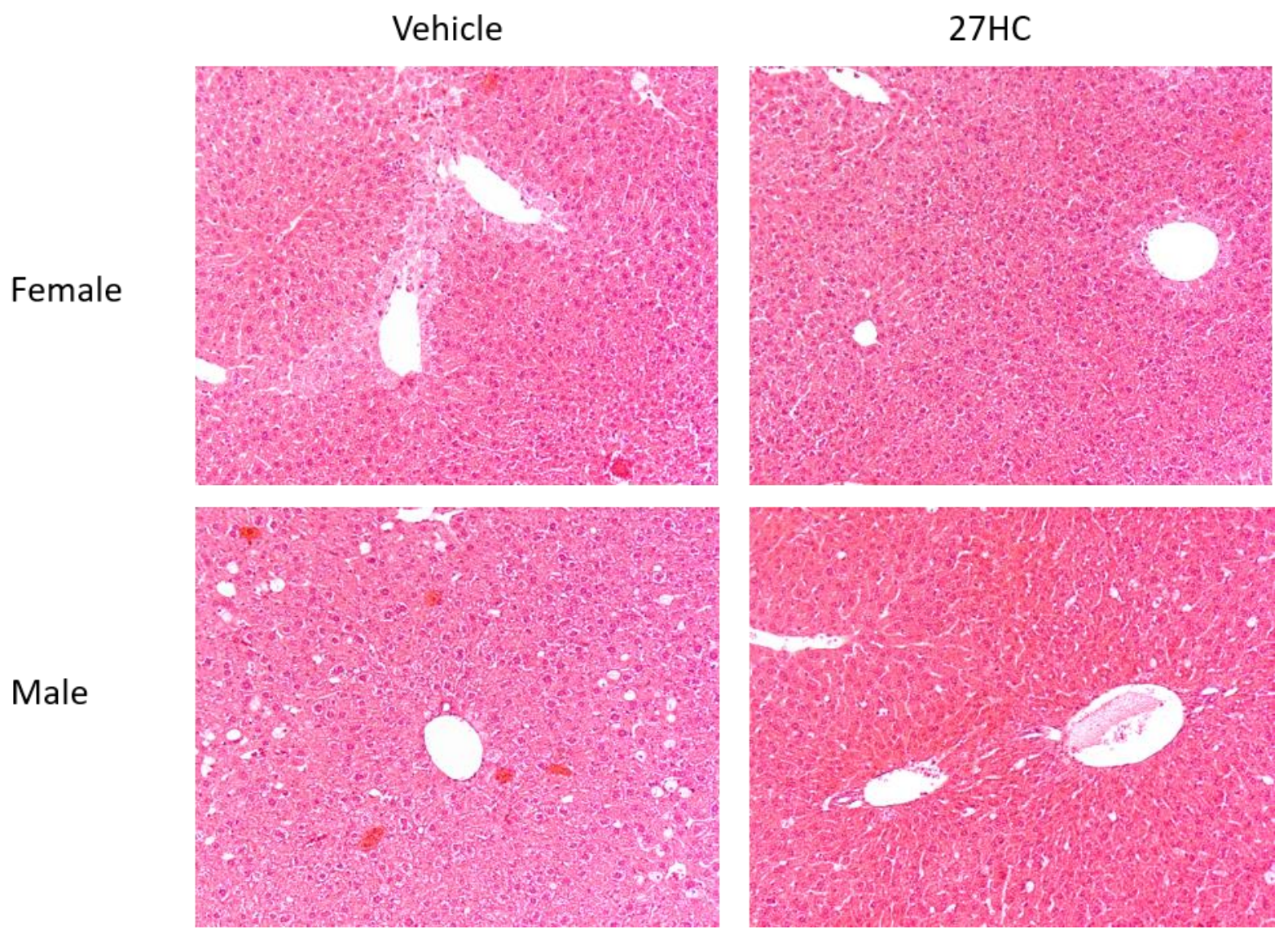

Supplemental Figure 5: Hepatic HE staining of female and male Npc1 nih mice. Representative images of hepatic HE staining (100x magnification) of female and male Npc1nih mice that were treated with vehicle or $27 \mathrm{HC}$ for 12 weeks. 


\section{Chapter 4}

A

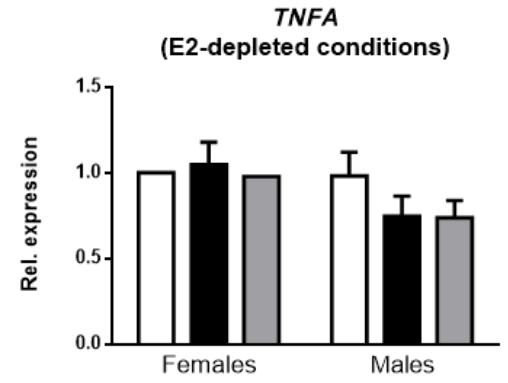

ICAM

(E2-depleted conditions)

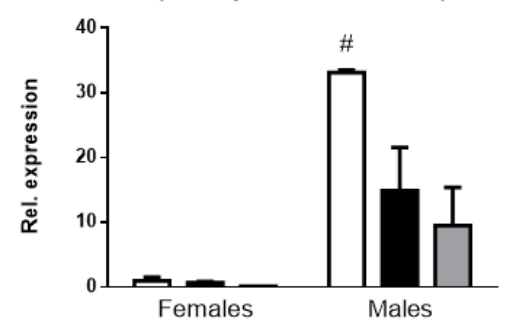

IL1B

(E2-depleted conditions)

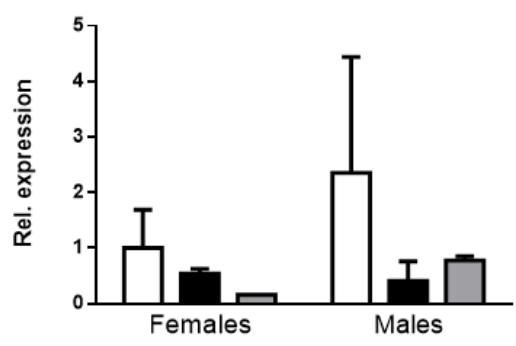

B

IL-10 secretion (E2-depleted conditions)

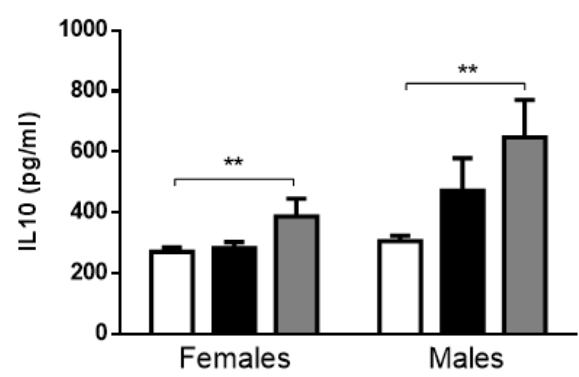

TNFA

(E2-enriched conditions)

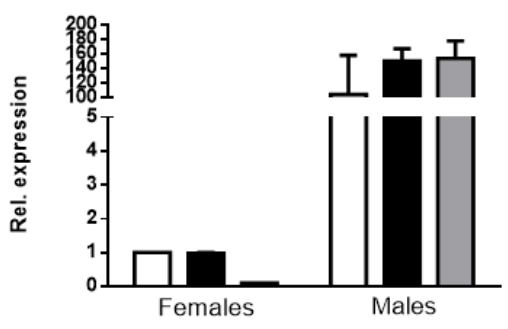

ICAM

(E2-enriched conditions)

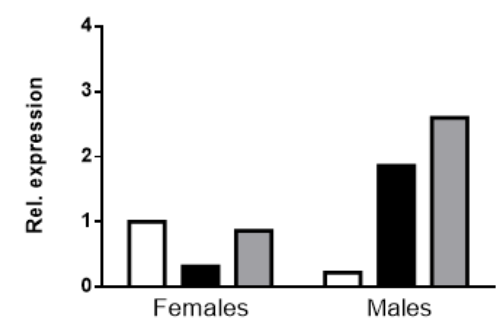

IL1B

(E2-enriched conditions)

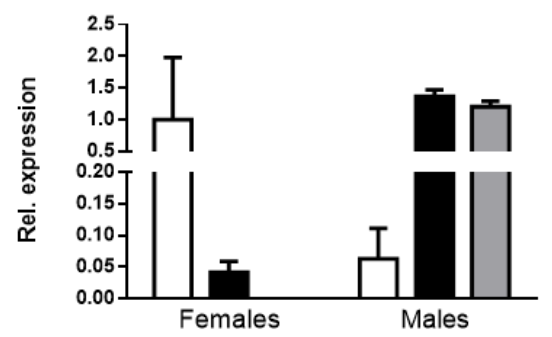

IL-10 secretion

(E2-enriched conditions)

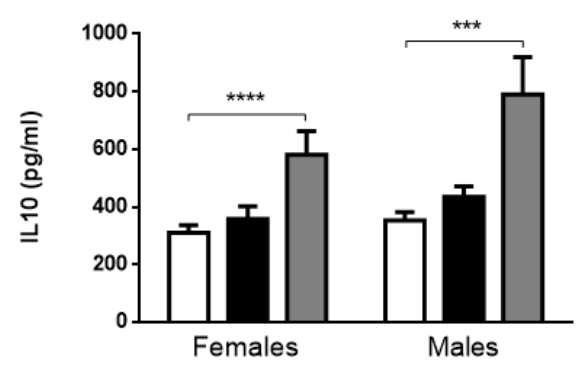

Supplemental Figure 6: IL-10 levels of female and male-derived Wt bone marrow-derived macrophages cultured in E2-enriched/depleted medium treated with 27HC. IL-10 protein levels in female and male Wt BMDMs. ** Indicates $\mathrm{p} \leq 0.01$ compared to vehicle-treated female- or male-derived BMDMs cultured under E2-depleted conditions by use of two-way ANOVA with Tukey's post-hoc correction (left panel). ${ }^{* * *} \mathrm{p} \leq 0.001$ and ${ }^{* * * *} \mathrm{p} \leq 0.0001$ is compared to vehicle-treated female or male BMDMs cultured under E2-enriched conditions (right panel). Results are shown as the average of 2 independent experiments. All error bars are SEM. 


\section{Chapter 4}

\section{Supplementary Table S1: Product information}

\begin{tabular}{|l|l|l|}
\hline Product & Company & Catalog number \\
\hline 27-hydroxycholesterol & Obtained via Prof. Plat & \\
\hline 2-hydroxypropyl-beta-cyclodextrin & Sigma-Aldrich & H107 \\
\hline Formaldehyde & Sigma-Aldrich & P6148-500g \\
\hline Chloroform & Merck & 1.02445 .1000 \\
\hline Isopropanol & Sigma-Aldrich & $33539-2.5 \mathrm{~L}$ \\
\hline Ethanol & Sigma-Aldrich & $32221-2.5 \mathrm{~L}$ \\
\hline DEPC sterile $\mathrm{H}_{2} \mathrm{O}$ & Sigma-Aldrich & D-5758 \\
\hline iScript cDNA synthesis & Bio-Rad & $170-8891$ \\
\hline Acetone & VWR & 20066.296 \\
\hline $\mathrm{H}_{2} \mathrm{O}_{2}$ & Merck & 1.07210 .1000 \\
\hline Fetal calf serum & Bodinco & BDS-12251 \\
\hline ABC kit & Vectastain & PK-6100 \\
\hline Mac1 antibody; rat-anti-mouse & Biolegend & 101207 \\
\hline rat-anti-mouse Ly6-C, NIMP & Hycult Biotech & HM1039PE-100 \\
\hline anti-rat-PO secondary antibody & DAKO & P0450 \\
\hline Anti-rat-BIO secondary antibody & Southern Biotech & 6180.08 \\
\hline Normal mouse serum & Invitrogen & 31881 \\
\hline AEC kit & Bio-connect & A85SK-4200.S1 \\
\hline Hematoxylin & Klinipath & 4085.9002 \\
\hline Faramount aqueous mounting medium & DAKO & S302580 \\
\hline Entallan & Merck & 1.07961 .1000 \\
\hline RPMI-1640 & GIBCO Invitrogen & $61870-010$ \\
\hline Penicillin \& Streptomycin & GIBCO Invitrogen & $15140-148$ \\
\hline L-glutamine & GIBCO Invitrogen & A2916801 \\
\hline 24-well plates & Greiner & 662102 \\
\hline Stripped estrogen serum & GIBCO & A33821-01 \\
\hline Estradiol & Sigma-Aldrich & E2758-250MG \\
\hline $\begin{array}{l}\text { Naphthylethylenediamine } \\
\text { dihydrochloride }\end{array}$ & Sigma-Aldrich & N9125 \\
\hline Sulfanilamide & Sigma-Aldrich & S9251 \\
\hline Phosphoric acid & Merck & 1.00573 .1000 \\
\hline Tumor necrosis factor alpha ELISA kit & Invitrogen & 176448001 \\
\hline Interleukin 10 ELISA kit & Invitrogen & $218449-002$ \\
\hline LXRb ELISA kit & Medical Supply company & MOEB0190 \\
\hline Estrogen receptor alpha & Elabscience & E-EL-M0476 \\
\hline Estrogen receptor beta & Elabscience & E-EL-M0490 \\
\hline & & \\
\hline
\end{tabular}




\section{Chapter 4}

Supplementary Table S2: Primer sequences for qRT-PCR

\begin{tabular}{|c|c|c|}
\hline Gene & Primer forward & Primer reverse \\
\hline TNFA & CATCTTCTCAAAATTCGAGTGACAA & $\begin{array}{l}\text { TGGGAGTAGACAAGGTACAACC } \\
\text { C }\end{array}$ \\
\hline CXCL2 & AGTGAACTGCGCTGTCAATGC & AGGCAAACTTTTTGACCGCC \\
\hline CD68 & TGACCTGCTCTCTCTAAGGCTACA & TCACGGTTGCAAGAGAAACATG \\
\hline ITGAM & ACTTTCAGAAGATGAAGGAGTTTGTCT & TGTGATCTTGGGCTAGGGTTTC \\
\hline ICAM & CTACCATCACCGTGTATTCGTTTC & CGGTGCTCCACCATCCA \\
\hline IL18 & GACTCTTGCGTCAACTTCAAGG & CAGGCTGTCTTTTGTCAACGA \\
\hline Il1B & $\begin{array}{l}\text { AAAGAATCTATACCTGTCCTGTGTAAT } \\
\text { GAAA }\end{array}$ & GGTATTGCTTGGGATCCACACT \\
\hline LXRB & AAGCAGGTGCCAGGGTTCT & $\begin{array}{l}\text { GTTTCTAGCAACATGATCTCAAT } \\
\text { GGT }\end{array}$ \\
\hline
\end{tabular}




\section{Chapter 4}

Supplementary Table S3: Population characteristics Maastricht cohort

\begin{tabular}{l|c|c} 
& Females & Males \\
\hline $\mathrm{n}$ & 21 & 13 \\
\hline Age (years) & $43.76 \pm 2.16$ & $45.62 \pm 2.33$ \\
\hline BMI (kg/m²) & $45.03 \pm 1.68$ & $44.70 \pm 2.47$ \\
\hline Waist/hip ratio & $0.98 \pm 0.06$ & $1.11 \pm 0.02$ \\
\hline Plasma total cholesterol (mg/dl) & $184.3 \pm 9.82$ & $159.3 \pm 6.60$ \\
\hline Plasma HDL (mg/dl) & $39.22 \pm 3.32$ & $34.03 \pm 2.87$ \\
\hline Plasma LDL (mg/dl) & $138.9 \pm 6.13$ & $113.7 \pm 10.77 *$ \\
\hline Plasma triglycerides (mg/dl) & $187.7 \pm 25.67$ & $142.2 \pm 27.06$
\end{tabular}

Data are represented as mean \pm SEM

*Indicates $p \leq 0.05$ compared to females by use of two-tailed unpaired $t$ test

Supplementary Table S4: Characteristics of seven week-old $\mathrm{Npc1}^{\text {nih }}$ mice

\begin{tabular}{l|c|c} 
& Females & Males \\
\hline $\mathrm{n}$ & 4 & 4 \\
\hline Total weight $(\mathrm{g})$ & $10.73 \pm 0.80$ & $12.43 \pm 0.93$ \\
\hline Relative liver weight & $0.08 \pm 0.002$ & $0.09 \pm 0.006$ \\
\hline Relative spleen weight & $0.006 \pm 0.001$ & $0.007 \pm 0.001$ \\
\hline Plasma total cholesterol (mg/dl) & $102.0 \pm 1.56$ & $112.8 \pm 17.58$ \\
\hline Plasma triglycerides $(\mathrm{mg} / \mathrm{dl})$ & $26.76 \pm 3.35$ & $43.35 \pm 11.36$ \\
\hline Liver total cholesterol $(\mathrm{mg} / \mathrm{dl})$ & $0.02 \pm 0.001$ & $0.03 \pm 0.001$ \\
\hline Liver total triglycerides $(\mathrm{mg} / \mathrm{dl})$ & $0.005 \pm 0.0008$ & $0.007 \pm 0.0005$ \\
& &
\end{tabular}

Data are represented as mean $\pm \mathrm{SEM}$ 


\section{Chapter 5}

Low profile high value target: the role of OxLDL in cancer 


\section{Chapter 5}

Chapter 5 Low profile high value target: the role of OxLDL in cancer

Albert V. Bitorina", Yvonne Oligschlaeger", Ronit Shiri-Sverdlov*, Jan Theys*

\#Contributed equally

Biochim Biophys Acta Mol Cell Biol Lipids. 2019 Dec;1864(12):158518 


\title{
Chapter 5
}

\begin{abstract}
Unhealthy Western-type diet and physical inactivity are highly associated with the current obesity epidemic and its related metabolic diseases such as atherosclerosis and non-alcoholic steatohepatitis. In addition, increasing evidence indicates that obesity is also a major risk factor for several types of common cancers. Recent studies have provided correlative support that disturbed lipid metabolism plays a role in cancer risk and development, pointing towards parallels in metabolic derangements between metabolic diseases and cancer. An important feature of disturbed lipid metabolism is the increase in circulating low-density lipoproteins, which can be oxidized (oxLDL). Elevated oxLDL and the level of its receptors have been positively associated with increased risk of various types of cancer. This review discusses the pro-oncogenic role of oxLDL in tumor development, progression and potential therapies, and provides insights into the underlying mechanisms.
\end{abstract}




\section{Chapter 5}

\section{Introduction}

According to the World Health Organization (WHO), it has been estimated that the global burden of cancer has risen to 18.1 million new cases and 9.6 million deaths in 2018, with an estimated 5-year prevalence of 43.8 million, putting cancer as the second leading cause of deaths worldwide (1). In parallel, the prevalence of risk factors for metabolic syndrome, such as overweight and obesity, have reached pandemic levels (2). Recent clinical data suggest that individuals with obesity are at an increased risk of developing several types of cancer with 5-7\% of new cancer cases attributed to overweight and diabetes (3-6). Mechanisms linking obesity with increased cancer development, progression and decreased treatment response (7) include adipose tissue dysfunction, increased circulating levels of hormones, low-grade chronic inflammation and perturbed lipid metabolism (8).

In recent years, lipid metabolism has been accepted as one of the major metabolic pathways involved in many aspects of cancer cell function including signaling processes related to cell transformation and tumor development. Hence, in recent years, various studies explored the involvement of different types of lipids and/or related pathways in carcinogenesis, cancer progression, malignancy and metastasis. Relevantly, a prominent feature related to dysregulated lipid metabolism and inflammation is the increased production of oxidized cholesterol-rich low-density lipoproteins (oxLDL), which results from elevated oxidative stress. Reactive oxygen species (ROS) are by-products of cellular metabolism, particularly generated by mitochondrial respiration (9). Normal cellular levels of ROS play an active role in regulating cell signaling pathways governing growth, proliferation, survival, angiogenesis and metabolism through increased expression of oncogenic genes such as hypoxia-inducible factor 1-alpha (HIF1- $\alpha$ ), NF-kb, phosphoinositide 3-kinases (PI3K) and mitogen-activated protein kinase (MAPK) $(10,11)$. Low physiological levels of ROS are maintained through active scavenging by antioxidants. However, in case the production of ROS exceeds cellular antioxidant scavenging capacity, oxidative stress occurs, leading to several pathological disorders, such as cardiovascular and neurodegenerative diseases (12). High levels of oxidative stress result in damage to cellular macromolecules such as proteins, DNA and lipids $(13,14)$. In the latter, changes can be induced in lipids carried by lipoproteins as well as in lipoproteins themselves. As such, low-density lipoproteins (LDL) can become susceptible to oxidative modifications, for instance in plasma or when retained in the artery wall (15), leading to conversion of LDL into oxLDL. Increased levels of oxLDL are strongly correlated to various (metabolic) diseases. OxLDL for instance plays a primary role in metabolic syndrome (MetS)-related diseases such as atherosclerosis (16), in which the uptake and degradation of oxLDL by macrophages is considered the main event initiating the formation of cholesterol-enriched foam cells that play a crucial role in the pathogenesis of atherosclerosis $(15,17)$. Similarly, intracellular accumulation of oxLDL in liver resident macrophages contribute to inflammation and disease progression of nonalcoholic steatohepatitis (NASH) (18). Importantly, the risk factor oxLDL has also been implicated in many aspects of cancer. This is exemplified by various studies showing increased levels of serum 


\section{Chapter 5}

oxLDL in patients with breast, pancreas, colon or esophageal cancer (19). Additionally, oxLDL has been shown to induce mutagenesis, stimulate proliferation, initiate metastasis and induce treatment resistance $(20,21)$. Mechanisms underlying these pro-oncogenic effects include epithelial-tomesenchymal transition (EMT), induction of protective autophagic responses, activation of the inflammasome and stimulation of survival pathways through the release of growth factors, cytokines and other pro-inflammatory markers $(20,22)$.

Given that increasing evidence unequivocally underlines the contribution of oxLDL to the development and progression of cancer, in the current review we will provide insights into the role of oxLDL in cancer pathology highlighting the effects of oxLDL on oncogenesis, cancer progression and metastasis. Finally, we will evaluate the impact of oxLDL on cancer treatment, and hence, explore possibilities for future intervention strategies.

\section{OxLDL and carcinogenesis}

Animal experiments as well as human epidemiological data have provided ample evidence that fatenriched diets and lipid-related diseases increase the incidence of certain types of cancer, such as breast, prostate, colon and liver cancer (23-25). In that context, plasma lipids have been shown to correlate with several types of cancers, as exemplified by clinical pathological studies demonstrating that plasma levels of total cholesterol (TC), triglycerides (TG) and LDL were significantly higher in breast cancer patients compared to healthy controls (26-28).

High fat diet can induce a switch towards a favorable microenvironment that supports the development and growth of cancer cells. Such a switch can be achieved through changes in hormonal environment or the properties of cell membranes, the latter due to alterations in lipid composition, or by means of modulating immune responses to tumor cells $(29,30)$. In addition, similar to observations in lipidrelated metabolic diseases such as obesity and NASH, oxidative stress will result in oxidation of LDL to oxLDL, leading to a plethora of pro-mutagenic and pro-carcinogenic effects (31). Various clinical studies underscore the role of oxLDL in the carcinogenic process and positive correlations between increased serum oxLDL concentration and cancer risk have been reported for pancreatic, colon, breast as well as esophageal cancer (32-35). Additionally, in a clinical study of colorectal cancer (CRC) patients, products of lipid oxidation as a consequence of oxidative stress, including oxLDL, were studied as potential markers of CRC development. Although this study did not observe significant differences in oxLDL serum levels between CRC patients and healthy controls, levels of oxLDL were shown to be significantly higher in patients with an early stage of primary tumor compared to patients with advanced stage primary tumor progression (19). 


\section{Chapter 5}

The human body is capable of producing autoantibodies against oxLDL (referred to as o-LAB), which can aid in the clearance of oxLDL from the blood (36). In accordance with the study of Suzuki et al., who showed a positive correlation between serum oxLDL and increased CRC risk, Diakowska et al. also showed that o-LAB plasma levels in patients with CRC were significantly increased compared to healthy controls and correlated with plasma levels of oxLDL. In line, oxLDL levels have also been shown to be significantly increased in patients with breast or ovarian cancer compared to healthy controls (37). These clinical observations reinforce the notion that oxLDL and its antibodies may play a crucial role in carcinogenesis, an observation further supported by data of Esterbauer et al., who showed that components of oxLDL such as 4-hydroxynonenal could stimulate mutagenesis in vitro. In this study, sub-cytotoxic levels of 4-hydroxynonenal administered to primary rat hepatocytes led to increases in micronuclei, chromosomal aberrations and sister-chromatid exchange, indicating increased DNA damage (38). In addition, it has been shown that oxLDL can potentially contribute to induction of cancer by increasing the expression of microRNA-210 (miR-210) (39). MiR-210 is regulated by HIF$1 \alpha$ and is known to have tumorigenic effects through modulation of cell cycle regulation, DNA damage repair, mitochondrial metabolism, tumor growth, apoptosis and angiogenesis (40). MiR210 has also been shown to inhibit sprouty-related EVH1 domain 2 (SPRED2), a protein that reduces cell migration. Chen et al. found increased miR-210 and reduced SPRED2 levels in aortas of mice fed with a high-fat diet as well as in tumor tissues, suggesting that miR-210 signaling can be an underlying mechanism to explain oxLDL as a common risk factor for cardiovascular disease and gastrointestinal cancer.

Recent evidence suggests that the receptors for oxLDL, i.e., cluster of differentiation 36 (CD36) and Lectin-Like Oxidized LDL Receptor-1 (LOX-1) are upregulated in and contribute to pathophysiology of dyslipidemia-related diseases such as cardiovascular disease and obesity $(41,42)$. Although these receptors are also known to be overexpressed in a variety of cancers and have been associated with increased cancer progression (see next section), there is currently no data available on whether overexpression of these receptors increase the risk of cancer, although that link may be evident. Future research is warranted to establish a link between these receptors and cancer risk. Collectively, these studies demonstrate clear associations between oxLDL and its antibodies with carcinogenic processes (Figure 1) in several types of cancer and underscore the close relationship between metabolic diseases and cancer. 


\section{Chapter 5}

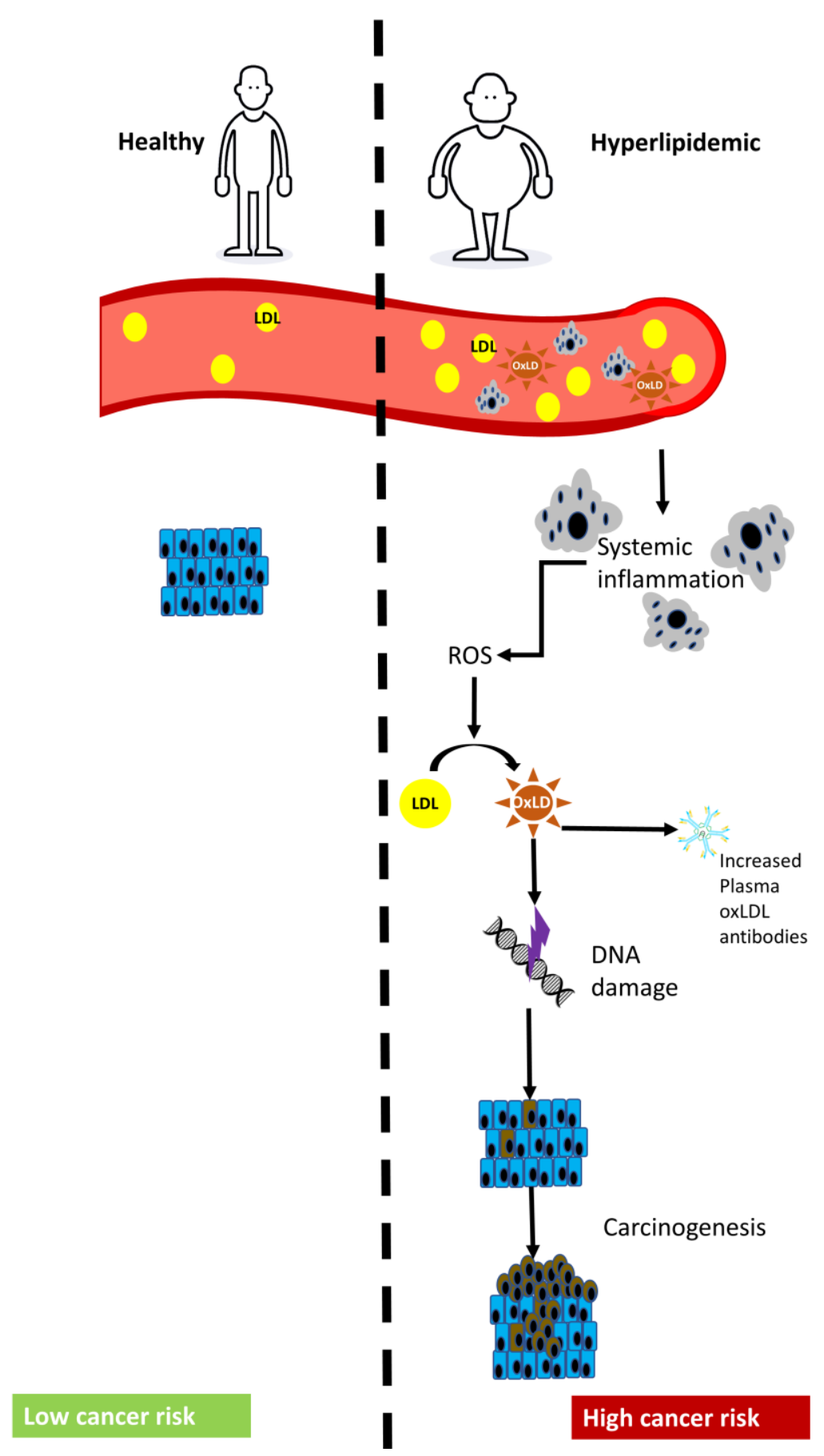

Figure 1 Carcinogenesis: Hyperlipidemic individuals have increased levels of plasma lipids including LDL in addition to elevated systemic inflammation. High systemic inflammation is often paired with oxidative stress leading to increased reactive oxygen species (ROS). In turn, high levels of ROS transform LDL into oxidized LDL (oxLDL), which can have mutagenic effects including damage to DNA. Elevated levels of oxLDL increases the risk of carcinogenesis. Furthermore, elevated plasma levels of oxLDL can lead to increased titers of natural antibodies against oxLDL, which may have diagnostic values for cancer risk. 


\section{Chapter 5}

\section{OxLDL and cancer progression}

Besides playing an important role during the onset of cancer, oxLDL has been shown to exert stimulating effects on cancer cell proliferation, thereby promoting cancer progression. Using quiescent human fibroblasts, Zettler et al. demonstrated that oxLDL can serve as an independent mitogenic factor increasing the expression of cell cycle-activating proteins, which in turn stimulated cell growth (43). These data are in line with Hale et al. showing that administration of oxLDL, rather than LDL, significantly increased the proliferation of patient-derived glioblastoma xenografts, explicitly by stimulating expansion of the cancer stem cell population within the tumor (44). Similarly, Scoles et al. demonstrated that treatment of ovarian carcinoma cells with oxLDL, but not LDL, increased cell proliferation (21). These data support the effect of oxLDL on promoting tumor growth. Curiously the proliferative effect of oxLDL was reportedly reversed by using an agonist of the Liver $\mathrm{x}$ Receptor (LXR) which is an oxysterol nuclear receptor (21). In general, LXR activation leads to increased cellular expression of cholesterol efflux proteins. Particularly in immune cells residing in the tumor microenvironment, LXR activation leads to decreased expression of several proinflammatory cytokines that possibly promote proliferation of cancer cells $(45,46)$. Additionally, in cancer cells, LXR activation has also been shown to inhibit proliferation $(47,48)$. However, while oxLDL is loaded with oxysterols and therefore can activate LXR and lead to increased cholesterol transport (49) it is also known to induce proinflammatory effects in macrophages (50). As oxLDL and LXR affect inflammation in an opposite manner, it is unlikely that the effect of oxLDL on proliferation is mediated via LXR signaling. Therefore, it can be inferred that oxLDL may exert its effect on proliferation of both cancer and immune cells in the tumor microenvironment through an LXR-independent manner.

In addition to the direct impact of oxLDL on cancer growth, recent studies also point towards a pivotal role for oxLDL-related receptors and the related downstream signaling pathways in the progression of cancer. Lipid-associated membrane receptors such as CD36 and LOX-1 are both important signaling membrane transporters involved in the uptake of oxLDL (51). Strikingly, genomic and proteomic profiling showed increased expression of these receptors in several cancer types as compared to their normal tissue counterparts $(52,53)$. In this context, CD36 was found to be overexpressed in glioblastoma patients compared to control. Furthermore, addition of oxLDL was shown to stimulate the proliferation of glioblastoma patient-derived xenografts, whereas siRNA-mediated knockdown of CD36 resulted in reduced proliferation (44). Similarly, overexpression of LOX-1 has particularly been demonstrated in the more advanced stages III and IV of human prostatic adenocarcinomas, suggesting an active role for LOX-1 specifically in disease progression (54). Overexpression of LOX-1 has also been observed in several other metabolic diseases including endothelial dysfunction, atherosclerosis and obesity $(55,56)$. In these disorders, binding of oxLDL to LOX-1 resulted in increased cell proliferation, cell motility and angiogenesis through the activation of Nf-Kb target genes such as vascular endothelial growth factor (VEGF), metalloproteinase-2 (MMP-2) and metalloproteinase-9 


\section{Chapter 5}

(MMP-9). Furthermore, it has been shown that oxLDL-mediated activation of such targets downstream of LOX-1 occurs in a dose-dependent manner. The observed increased target gene expression in turn enhances tumor angiogenesis in human prostate cancer cells facilitating tumor growth (57) and is potentially associated with the process of metastasis. Indeed, in addition to the role of oxLDL and its aforementioned receptors in cancer initiation and proliferation, recent evidence also points to a role for oxLDL and its receptors in the spreading of tumor cells through metastasis. Metastasis is a multistep process including: the acquisition of migratory and invasive abilities in the primary cancer site, entering of cancer cells into the bloodstream, exiting of cancer cells into the parenchyma of distant organs or tissues, and lastly cancer cell survival and proliferation to form macro-metastases (58). Metastatic cancers are responsible for more than $90 \%$ of all cancer-related deaths (59), often due to the difficulties in surgical resection or treatment with conventional radiation and/or chemotherapy. Recently, a striking link between oxLDL and lymphatic vessel density has been found in the context of gastric cancer. Data from this study showed that oxLDL promoted lymphatic metastasis of gastric cancer in nude mice. These findings were also confirmed by clinical data demonstrating that plasma oxLDL levels positively correlated with lymphatic metastasis in patients with gastric cancer (60).

A growing number of studies have recently shown that overexpression of CD36 also plays a prominent role in enabling cancer metastasis and therefore could be considered a biomarker of malignancy (44). The metastatic spreading of cancer cells is known to rely on CD36-mediated exogenous fatty acid (FA) uptake and metabolism through the fatty acid $\beta$-oxidation (FAO) pathway. Mechanistically, this implies that CD36-positive cells utilize FAO to more efficiently obtain the required energy to sustain and survive through the process of metastasis (59). In line, it has been shown that cells derived from human oral carcinomas express high levels of CD36, other lipid-related genes and initiate metastasis in a CD36dependent manner. More specifically, palmitic acid or a high-fat diet was shown to boost the metastatic potential of these metastasis-initiating cells in a CD36-dependent manner (61).

An important mechanism through which cancer cells gain the ability to metastasize is via the epithelialto-mesenchymal transition (EMT), a process that confers cancer cells migratory abilities through acquisition of a series of stem-like properties. Cancer cells that undergo EMT lose their cell-cell adhesion capabilities and break through the basal membrane to gain access to the bloodstream (62). It is established that EMT can be triggered through dysregulated metabolism by means of various alterations in the microenvironment such as hypoxia, acidification and glucose deprivation (63). The overall deregulated metabolism enables cancer cells to circumvent the nutrient and oxygen supply limitation caused by the rapid primary cancer growth that would otherwise bottleneck the progression process. Several studies on cancer cells have shown that overexpression of genes specifically related to lipid metabolism can lead to EMT induction, thereby increasing the migratory and invasive properties of these cells. For instance, hepatocellular carcinoma (HCC) progression via induction of EMT is highly associated with the expression of CD36 and elevated free fatty acid (FFA) levels (64). Furthermore, 


\section{Chapter 5}

Park et al. showed that the binding of oxLDL to CD36 increased the activation of focal adhesion kinase 1 (FAK1) and Ras-related C3 botulinum toxin substrate 1 (RAC1) (65). These proteins are in part responsible for the cellular morphological changes necessary for the initiation of EMT, including loss of polarity and actin polymerization (64). Other important metastatic events regulated by oxLDL are the expression and activation of MMPs and cytoskeletal restructuration (66). As previously mentioned, it was shown that oxLDL increased MMP-2/9 activity in a dose-dependent manner through its interaction with LOX-1 in prostate cancer cell lines. Likewise, LOX-1 is overexpressed in mammary and prostate cancer cells and its activation by oxLDL promotes EMT (66). Similar to prostate cancer, LOX-1 has also been suggested to play a role in the initiation of metastasis of gastric cancer (22).

Recent findings suggest autophagy as an important mechanism through which oxLDL contributes to EMT in cancer. Autophagy is a catabolic process which mediates the degradation of dysfunctional cellular components. This mechanism allows cells to eliminate damaged organelles in addition to potentially dangerous molecules such as cytostatic compounds, ensuring the maintenance of cellular homeostasis. Autophagy is also a powerful survival strategy capable of improving resistance to unfavorable conditions such as cell-intrinsic or environmental stressful stimuli (e.g. nutrient deprivation, hypoxia) that cells experience when metastasizing (67). In line, several studies show that alterations in the autophagic machinery promote the metastatic spreading of cancer (68-71). During metastasis, the reorganization of cell-cell interaction properties and complete loss of extracellular matrix adhesion leads to the induction of cell death pathways. Initiation of the EMT program also leads to these massive cellular changes, which expose cancer cells to strong cell-death initiating stimuli. By activating autophagy, cells undergoing EMT can acquire resistance to cell death as a strategy to survive while spreading outside the tumor mass (67). In line, it has recently been shown that autophagy is required for lung metastasis of HCC cells (72). In that study, it was demonstrated in an orthotopic mouse model that silencing of the autophagic factors Beclin1 (BCN1) and ATG5 in liver cancer cells impaired the incidence of lung metastases. Furthermore, inhibition of autophagy did not seem to affect the migration or invasive capacity of liver cancer cells, although resistance to cell death and colonization in the lungs was found to be reduced (72). Another study using HUVEC cells showed that oxLDL is capable of inducing autophagy in part through activation microRNA-155 (miR-155) (73). Indeed, inhibition of miR-155 lead to reduced numbers of autophagic bodies in HUVEC cells treated with oxLDL (74). These findings suggest induction of autophagy as an additional mechanism through which oxLDL contributes to EMT in cancer.

Taken together, there is growing evidence that the effects of oxLDL are not limited to associations with cancer initiation, but rather also involve effects on proliferation and metastatic processes (Figure 2), thereby contributing to cancer malignancy in a wide variety of cancers. 


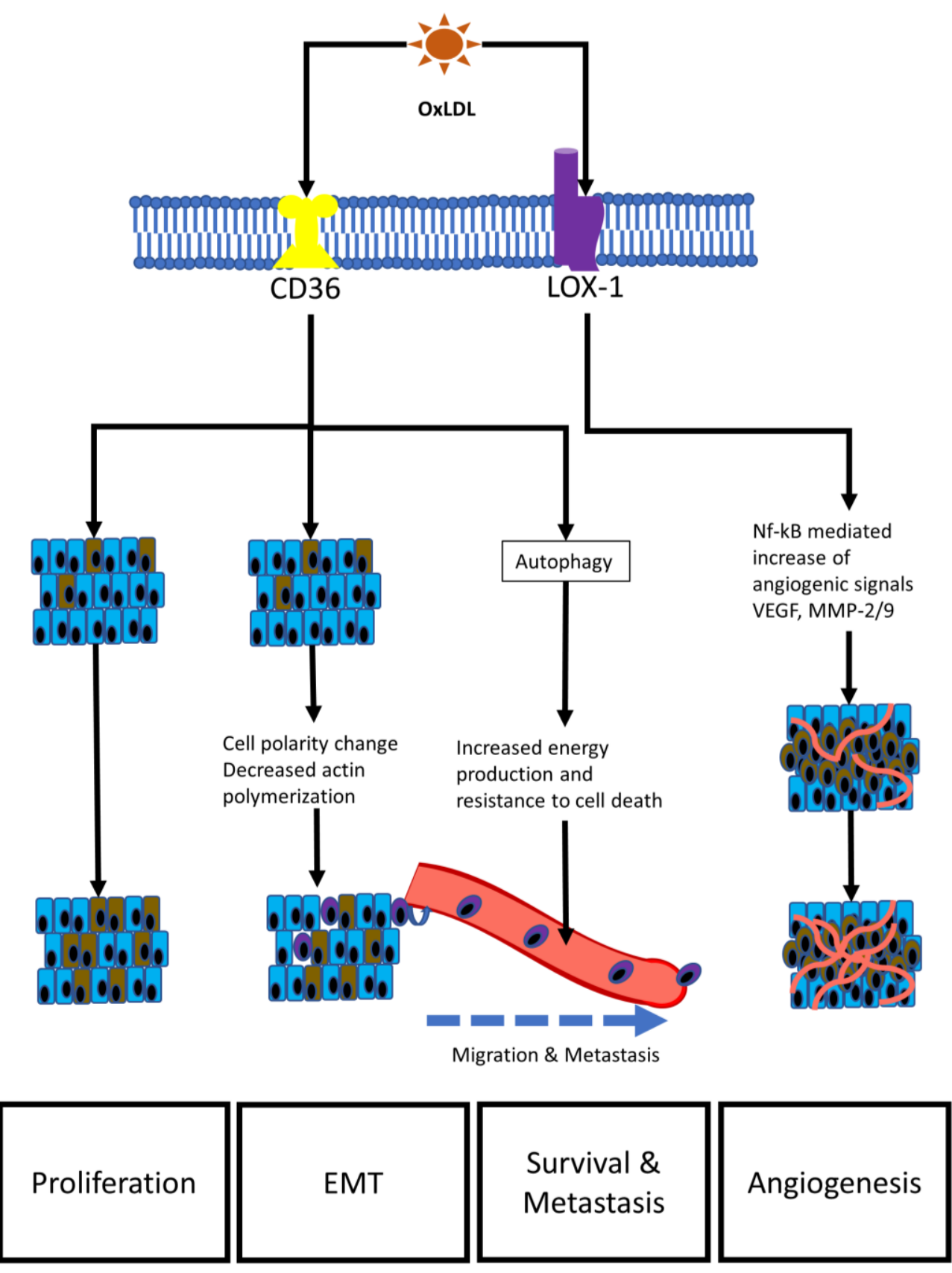

Figure 2 Cancer progression: OxLDL through interactions with its receptors CD36 or LOX-1 can lead to increased cell proliferation. CD36 mediated signaling can cause cancer cells to transform their phenotype to exhibit mesenchymal characteristics (EMT) and promote metastasis. During the stressful migration stage, cancer cells can utilize autophagy to meet energy requirements and insure survival. OxLDL signaling through LOX-1 can increase angiogenic factors in order to vascularize the tumor to gain access to sufficient nutrients. 


\section{Chapter 5}

\section{OxLDL and cancer treatment}

Based on its involvement in the onset, progression and metastasis of cancer, the role of oxLDL and related receptors has gained increased awareness also in the context of cancer treatment and outcome. The potential of targeting receptors related to oxLDL to reduce metastasis has been demonstrated in a variety of orthotopic cancer models including melanoma, oral, breast, and ovarian cancers (75). For instance, Pascual et al. demonstrated that therapeutic inhibition of CD36, which is critically involved in the uptake of oxLDL, almost completely abolished the ability of those cells to metastasize without overt systemic side effects (61). Likewise, Nath et al. showed that inhibiting the CD36 receptor fatty acid uptake also mitigated the EMT phenotype in human liver cancer cell lines (64). In this study, human liver cancer cells were treated with fatty acids and exhibited increased migration compared to cells treated with fatty acids and a CD36 inhibitor. Additionally, this study found that fatty acids enact their driving effects on EMT through CD36-mediated upregulation of Nf-kb, which in turn induces WNT and transforming growth factor (TGF) signaling pathways.

Apart from preventing metastasis, targeting oxLDL may also improve the outcome of current conventional therapies. Such an approach would be highly significant, as resistance to for instance chemotherapy or immunotherapy results in cancer progression and causes relapse. Few studies have looked into the role of lipids on therapy efficacy. For instance, Guillaumond et al. found a higher risk of pancreatic adenocarcinoma recurrence in patients with high LDL receptor expression and showed that blocking cholesterol uptake sensitized cells to chemotherapeutic drugs as well as strengthened the effect of chemotherapy on pancreatic adenocarcinoma regression (76). Similarly, several studies found that the use of statins, which are competitive inhibitors of the cholesterol synthesis enzyme 3-Hydroxy3-Methylglutaryl-CoA Reductase (HMGCR), as an adjuvant to chemotherapy, improved chemosensitivity in a variety of cancer cells (77). Likewise, statins have also been found to improve immunotherapy sensitivity by reducing immune cell exhaustion and thus reducing cancer-related mortality as well as tumor reoccurrence (78).

OxLDL has also been shown to affect chemosensitivity in vitro, as demonstrated by the findings of Scoles et al., where the administration of oxLDL increased the IC50 of cisplatin and hence reduced chemosensitivity in ovarian carcinoma cell lines, as determined by an MTT assay (21). It was previously addressed that oxLDL was capable of inducing the expression of miR-155, which in turn activated autophagy that can function as a survival mechanism for tumors. In addition, Chen et al. showed that autophagy induced by overexpression of miR-155 led to chemoresistance in osteosarcoma cells (79). In this study, osteosarcoma cells treated with miR-155 mimics reduced the efficacy of chemotherapy in a dose-dependent manner. This finding suggests oxLDL-induced autophagy as a potential mechanism through which oxLDL confers resistance to chemotherapy. Nevertheless, studies investigating the direct the impact of oxLDL on chemoresistance are warranted to confirm this observation. 


\section{Chapter 5}

Altogether, although observations directly linking oxLDL to treatment outcomes are limited, these data point towards an important role for oxLDL and its receptors in cancer treatment (Figure 3) and open venues to study the potential of anti-oxLDL approaches as an adjuvant to current standard treatment options. 


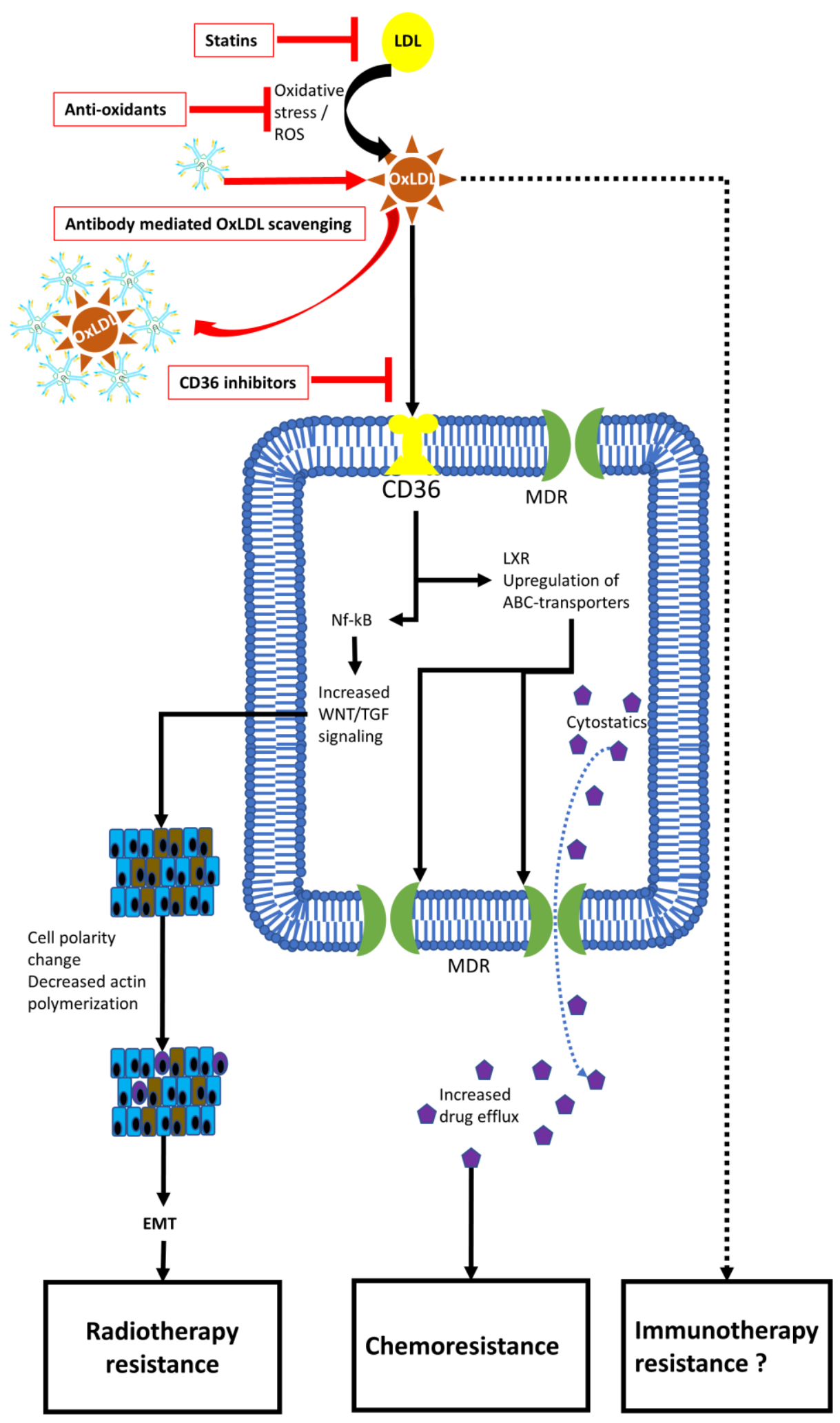

Figure 3 Cancer treatment: OxLDL can lead to CD36 signaling, thereby promoting EMT, a process known to induce radioresistance. Additionally, CD36 can upregulate the ABC-transporters (MDR), thereby promoting efflux of cytostatic drugs used in chemotherapy and hence inducing chemoresistance. Current treatment modalities include statins which can lower overall LDL cholesterol, anti-oxidants which through scavenging of ROS can reduce total plasma oxLDL, antibodies against oxLDL that can reduce oxLDL uptake by cancer cells and CD36 inhibitors, which can block downstream oxLDL signaling that confers treatment resistance. 


\section{Chapter 5}

\section{Discussion and Conclusion}

The involvement of lipids in the pathology of cancer has gained increasing interest in recent years. A growing body of evidence indicates a contribution of oxLDL to the development of cancer through for instance increased oxidative stress-induced cell damage, the progression of cancer through activation of proliferative pathways or induction of invasive phenotypes. In addition, few studies explored the possibilities of targeting oxLDL either directly as a treatment option or in the context of increasing efficacy of existing therapeutic modalities. OxLDL may thus be considered an attractive therapeutic target in cancer.

Given the potential role of oxLDL in metastasis and taking into account the importance of metastasis in defining prognosis and treatment outcome, targeting oxLDL may be beneficial in this context. We have previously established oxLDL as an attractive target in the pathology of NASH, in which targeting of oxLDL through the use of specific antibodies against oxLDL significantly improved liver lipid levels and reduced inflammation (50). These antibodies could potentially be tested for their ability to reduce metastatic spread. Yet, although several studies have pointed towards the promising potential of mitigating metastasis or EMT through inhibition of CD36, the direct impact of reducing oxLDL was never investigated. Since CD36 also has other important functions in the human body, such as fatty acid uptake in cardiac cells for energy metabolism $(80,81)$, direct targeting of oxLDL may be preferable to avoid probable side effects of long-term inhibition of CD36.

To our knowledge, only one study has investigated the direct role of oxLDL on treatment efficacy, showing increased chemotherapy resistance in the presence of oxLDL (21). One of the most prominent mechanisms of chemoresistance is multi drug resistance (MDR) and the main players are the MDR receptors, which are in fact ATP Binding Cassette (ABC)-transporters. Several members of this transmembrane receptor super family have been found to transport various chemotherapeutic agents out of the cell, thereby decreasing their effectiveness $(82,83)$. ABC-transporters have the primary function of shuttling cholesterol out of the cell and are regulated by cholesterol sensing proteins such as LXR. Although it is not yet known how this direct link between oxLDL or lipids in general and their influence on the expression of the $\mathrm{ABC}$ receptors influences the efficacy of chemotherapy, the available data indicate the potential for this research area to be explored. Relevantly, it is also known that HIF1- $\alpha$ stabilization can induce chemoresistance through upregulation of $\operatorname{MDR}(84,85)$. HIF1- $\alpha$ can be stabilized under both hypoxic as well as normoxic conditions. For instance, it has been shown in macrophages that oxLDL is capable of activating HIF1-a under normoxic conditions through redox mechanisms (86). As such, modulation of ABC transporters and stabilization of HIF1-a exemplify two more possible mechanisms along with the previously suggested effect on autophagy, through which oxLDL could have a direct impact on treatment outcome. More studies are needed that focus on the relation between lipids and the expression of MDR, looking into its correlation to chemotherapy 


\section{Chapter 5}

resistance. Additionally, attention should also be given to the potential involvement of oxLDL and its receptors in the area of immunotherapy, as recently LOX-1 was also found to be overexpressed in Polymorphonuclear myeloid-derived suppressor cells (PMN-MDSC). These cells are important regulators of the immune responses in cancer and are directly involved in stimulation of tumor progression (87).

In conclusion, in this review we have highlighted the intricate involvement of oxLDL in tumorigenesis, progression, metastasis as well as treatment. Though there is sufficient evidence to establish oxLDL as an important player in cancer, there is a current lack of knowledge particularly on the impact of oxLDL on treatment efficacy and the viability of targeting oxLDL as an adjuvant to standard care. Considering the enormous impact of treatment resistance on patient outcome, we suggest future studies to further explore this area in order to better understand cancer resistance mechanisms and improve treatment outcomes. 


\section{Chapter 5}

\section{References}

1. WHO. Press release 263 Latest global cancer data: Cancer burden rises to 18.1 million new cases and 9.6 million cancer deaths in 20182018 [Available from: https://www.iarc.fr/featurednews/latest-global-cancer-data-cancer-burden-rises-to-18-1-million-new-cases-and-9-6million-cancer-deaths-in-2018/.

2. Friedrich MJ. Global Obesity Epidemic Worsening. Jama. 2017;318(7):603.

3. Doerstling SS, O'Flanagan $\mathrm{CH}$, Hursting SD. Obesity and Cancer Metabolism: A Perspective on Interacting Tumor-Intrinsic and Extrinsic Factors. Frontiers in oncology. 2017;7:216-.

4. Chen Y, Wang X, Wang J, Yan Z, Luo J. Excess body weight and the risk of primary liver cancer: an updated meta-analysis of prospective studies. European journal of cancer (Oxford, England : 1990). 2012;48(14):2137-45.

5. Genkinger JM, Spiegelman D, Anderson KE, Bernstein L, van den Brandt PA, Calle EE, et al. A pooled analysis of 14 cohort studies of anthropometric factors and pancreatic cancer risk. International journal of cancer. 2011;129(7):1708-17.

6. The Editors Of The Lancet Diabetes E. Retraction and republication-Worldwide burden of cancer attributable to diabetes and high body-mass index: a comparative risk assessment. Lancet Diabetes Endocrinol. 2018;6(6):437.

7. Esposito K, Chiodini P, Colao A, Lenzi A, Giugliano D. Metabolic syndrome and risk of cancer: a systematic review and meta-analysis. Diabetes care. 2012;35(11):2402-11.

8. Micucci C, Valli D, Matacchione G, Catalano A. Current perspectives between metabolic syndrome and cancer. Oncotarget. 2016;7(25):38959-72.

9. Zorov DB, Juhaszova M, Sollott SJ. Mitochondrial reactive oxygen species (ROS) and ROSinduced ROS release. Physiological reviews. 2014;94(3):909-50.

10. Vadlapatla RK, Vadlapudi AD, Mitra AK. Hypoxia-inducible factor-1 (HIF-1): a potential target for intervention in ocular neovascular diseases. Curr Drug Targets. 2013;14(8):919-35.

11. Zhang J, Wang X, Vikash V, Ye Q, Wu D, Liu Y, et al. ROS and ROS-Mediated Cellular Signaling. Oxid Med Cell Longev. 2016;2016:4350965.

12. Liguori I, Russo G, Curcio F, Bulli G, Aran L, Della-Morte D, et al. Oxidative stress, aging, and diseases. Clin Interv Aging. 2018;13:757-72. 


\section{Chapter 5}

13. Lobo V, Patil A, Phatak A, Chandra N. Free radicals, antioxidants and functional foods: Impact on human health. Pharmacogn Rev. 2010;4(8):118-26.

14. Schieber M, Chandel NS. ROS function in redox signaling and oxidative stress. Curr Biol. 2014;24(10):R453-62.

15. Lu M, Gursky O. Aggregation and fusion of low-density lipoproteins in vivo and in vitro. Biomol Concepts. 2013;4(5):501-18.

16. Di Pietro N, Formoso G, Pandolfi A. Physiology and pathophysiology of oxLDL uptake by vascular wall cells in atherosclerosis. Vascular pharmacology. 2016;84:1-7.

17. Jeurissen MLJ, Walenbergh SMA, Houben T, Gijbels MJJ, Li J, Hendrikx T, et al. Prevention of oxLDL uptake leads to decreased atherosclerosis in hematopoietic NPC1-deficient Ldlr(-/-) mice. Atherosclerosis. 2016;255:59-65.

18. Walenbergh SM, Koek GH, Bieghs V, Shiri-Sverdlov R. Non-alcoholic steatohepatitis: the role of oxidized low-density lipoproteins. J Hepatol. 2013;58(4):801-10.

19. Diakowska D, Grabowski K, Nienartowicz M, Zarebski P, Fudalej K, Markocka-Maczka K. Circulating Oxidized Low-Density Lipoproteins and Antibodies against Oxidized Low-Density Lipoproteins as Potential Biomarkers of Colorectal Cancer. Gastroenterol Res Pract. 2015;2015:146819.

20. Zabirnyk O, Liu W, Khalil S, Sharma A, Phang JM. Oxidized low-density lipoproteins upregulate proline oxidase to initiate ROS-dependent autophagy. Carcinogenesis. 2010;31(3):446-54.

21. Scoles DR, Xu X, Wang H, Tran H, Taylor-Harding B, Li A, et al. Liver X receptor agonist inhibits proliferation of ovarian carcinoma cells stimulated by oxidized low density lipoprotein. Gynecol Oncol. 2010;116(1):109-16.

22. Li C, Zhang J, Wu H, Li L, Yang C, Song S, et al. Lectin-like oxidized low-density lipoprotein receptor-1 facilitates metastasis of gastric cancer through driving epithelial-mesenchymal transition and PI3K/Akt/GSK3beta activation. Sci Rep. 2017;7:45275.

23. Chimento A, Casaburi I, Avena P, Trotta F, De Luca A, Rago V, et al. Cholesterol and Its Metabolites in Tumor Growth: Therapeutic Potential of Statins in Cancer Treatment. Front Endocrinol (Lausanne). 2018;9:807.

24. Koh WP, Dan YY, Goh GB, Jin A, Wang R, Yuan JM. Dietary fatty acids and risk of hepatocellular carcinoma in the Singapore Chinese health study. Liver Int. 2016;36(6):893-901. 


\section{Chapter 5}

25. Ioannou GN, Morrow OB, Connole ML, Lee SP. Association between dietary nutrient composition and the incidence of cirrhosis or liver cancer in the United States population. Hepatology (Baltimore, Md). 2009;50(1):175-84.

26. Laisupasin P, Thompat W, Sukarayodhin S, Sornprom A, Sudjaroen Y. Comparison of Serum Lipid Profiles between Normal Controls and Breast Cancer Patients. J Lab Physicians. 2013;5(1):38-41.

27. Owiredu WK, Donkor S, Addai BW, Amidu N. Serum lipid profile of breast cancer patients. Pak J Biol Sci. 2009;12(4):332-8.

28. Raza U, Asif MR, Rehman AB, Sheikh A. Hyperlipidemia and hyper glycaemia in Breast Cancer Patients is related to disease stage. Pak J Med Sci. 2018;34(1):209-14.

29. Baenke F, Peck B, Miess H, Schulze A. Hooked on fat: the role of lipid synthesis in cancer metabolism and tumour development. Dis Model Mech. 2013;6(6):1353-63.

30. Robinson GA, Waddington KE, Pineda-Torra I, Jury EC. Transcriptional Regulation of T-Cell Lipid Metabolism: Implications for Plasma Membrane Lipid Rafts and T-Cell Function. Front Immunol. 2017;8:1636.

31. Khaidakov M, Mehta JL. Do atherosclerosis and obesity-associated susceptibility to cancer share causative link to oxLDL and LOX-1? Cardiovascular drugs and therapy. 2011;25(5):47787.

32. Delimaris I, Faviou E, Antonakos G, Stathopoulou E, Zachari A, Dionyssiou-Asteriou A. Oxidized LDL, serum oxidizability and serum lipid levels in patients with breast or ovarian cancer. Clinical biochemistry. 2007;40(15):1129-34.

33. Suzuki K, Ito Y, Wakai K, Kawado M, Hashimoto S, Toyoshima H, et al. Serum oxidized lowdensity lipoprotein levels and risk of colorectal cancer: a case-control study nested in the Japan Collaborative Cohort Study. Cancer epidemiology, biomarkers \& prevention : a publication of the American Association for Cancer Research, cosponsored by the American Society of Preventive Oncology. 2004;13(11 Pt 1):1781-7.

34. Diakowska D, Grabowski K, Nienartowicz M, Markocka-Mạczka K. Lipid peroxidation and total antioxidant status in upper gastrointestinal tract cancers2007. 405-8 p.

35. Kodydkova J, Vavrova L, Stankova B, Macasek J, Krechler T, Zak A. Antioxidant status and oxidative stress markers in pancreatic cancer and chronic pancreatitis. Pancreas. 2013;42(4):614-21. 


\section{Chapter 5}

36. Huang $\mathrm{CH}$, Chang $\mathrm{CC}$, Huang $\mathrm{CS}$, Kuo $\mathrm{CL}$, Chen $\mathrm{CP}$, Hsia $\mathrm{CH}$, et al. Using oxidized lowdensity lipoprotein autoantibodies to predict restenosis after balloon angioplasty in patients with acute myocardial infarction. PloS one. 2013;8(10):e74726.

37. Siti ZS, Seoparjoo AMI, Shahrul H. Lipoproteins modulate growth and P-glycoprotein expression in drug-resistant HER2-overexpressed breast cancer cells. Heliyon. 2019;5(4):e01573.

38. Esterbauer H, Eckl P, Ortner A. Possible mutagens derived from lipids and lipid precursors. Mutation research. 1990;238(3):223-33.

39. Liu Y, Wang Y, Xu Q, Zhou X, Qin Z, Chen C, et al. Prognostic evaluation of microRNA-210 in various carcinomas: Evidence from 19 studies. Medicine (Baltimore). 2017;96(43):e8113-e.

40. Chen KC, Liao YC, Wang JY, Lin YC, Chen CH, Juo SH. Oxidized low-density lipoprotein is a common risk factor for cardiovascular diseases and gastroenterological cancers via epigenomical regulation of microRNA-210. Oncotarget. 2015;6(27):24105-18.

41. Chistiakov DA, Orekhov AN, Bobryshev YV. LOX-1-Mediated Effects on Vascular Cells in Atherosclerosis. Cellular Physiology and Biochemistry. 2016;38(5):1851-9.

42. Koonen DPY, Jacobs RL, Febbraio M, Young ME, Soltys C-LM, Ong H, et al. Increased Hepatic CD36 Expression Contributes to Dyslipidemia Associated With Diet-Induced Obesity. Diabetes. 2007;56(12):2863.

43. Zettler ME, Prociuk MA, Austria JA, Massaeli H, Zhong G, Pierce GN. OxLDL stimulates cell proliferation through a general induction of cell cycle proteins. American journal of physiology Heart and circulatory physiology. 2003;284(2):H644-53.

44. Hale JS, Otvos B, Sinyuk M, Alvarado AG, Hitomi M, Stoltz K, et al. Cancer stem cell-specific scavenger receptor CD36 drives glioblastoma progression. Stem Cells. 2014;32(7):1746-58.

45. Taniguchi K, Karin M. NF-kappaB, inflammation, immunity and cancer: coming of age. Nat Rev Immunol. 2018;18(5):309-24.

46. Bensinger SJ, Tontonoz P. Integration of metabolism and inflammation by lipid-activated nuclear receptors. Nature. 2008;454(7203):470-7.

47. Ju X, Huang P, Chen M, Wang Q. Liver X receptors as potential targets for cancer therapeutics. Oncol Lett. 2017;14(6):7676-80.

48. Lo Sasso G, Bovenga F, Murzilli S, Salvatore L, Di Tullio G, Martelli N, et al. Liver X receptors inhibit proliferation of human colorectal cancer cells and growth of intestinal tumors in mice. Gastroenterology. 2013;144(7):1497-507, 507.e1-13. 


\section{Chapter 5}

49. Chawla A, Boisvert WA, Lee CH, Laffitte BA, Barak Y, Joseph SB, et al. A PPAR gammaLXR-ABCA1 pathway in macrophages is involved in cholesterol efflux and atherogenesis. Mol Cell. 2001;7(1):161-71.

50. Houben T, Oligschlaeger Y, Bitorina AV, Hendrikx T, Walenbergh SMA, Lenders M-H, et al. Blood-derived macrophages prone to accumulate lysosomal lipids trigger oxLDL-dependent murine hepatic inflammation. Scientific Reports. 2017;7(1):12550.

51. Martin-Fuentes P, Civeira F, Recalde D, Garcia-Otin AL, Jarauta E, Marzo I, et al. Individual variation of scavenger receptor expression in human macrophages with oxidized low-density lipoprotein is associated with a differential inflammatory response. Journal of immunology (Baltimore, Md : 1950). 2007;179(5):3242-8.

52. Jia S, Zhou L, Shen T, Zhou S, Ding G, Cao L. Down-expression of CD36 in pancreatic adenocarcinoma and its correlation with clinicopathological features and prognosis. J Cancer. 2018;9(3):578-83.

53. Murdocca M, Mango R, Pucci S, Biocca S, Testa B, Capuano R, et al. The lectin-like oxidized LDL receptor-1: a new potential molecular target in colorectal cancer. Oncotarget. 2016;7(12):14765-80.

54. Hirsch HA, Iliopoulos D, Joshi A, Zhang Y, Jaeger SA, Bulyk M, et al. A transcriptional signature and common gene networks link cancer with lipid metabolism and diverse human diseases. Cancer Cell. 2010;17(4):348-61.

55. Chen M, Masaki T, Sawamura T. LOX-1, the receptor for oxidized low-density lipoprotein identified from endothelial cells: implications in endothelial dysfunction and atherosclerosis. Pharmacology \& therapeutics. 2002;95(1):89-100.

56. Brinkley TE, Kume N, Mitsuoka H, Phares DA, Hagberg JM. Elevated soluble lectin-like oxidized LDL receptor-1 (sLOX-1) levels in obese postmenopausal women. Obesity (Silver Spring). 2008;16(6):1454-6.

57. González-Chavarría I, Cerro RP, Parra NP, Sandoval FA, Zuñiga FA, Omazábal VA, et al. Lectin-like oxidized LDL receptor-1 is an enhancer of tumor angiogenesis in human prostate cancer cells. PloS one. 2014;9(8):e106219-e.

58. Lehuede C, Dupuy F, Rabinovitch R, Jones RG, Siegel PM. Metabolic Plasticity as a Determinant of Tumor Growth and Metastasis. Cancer research. 2016;76(18):5201-8.

59. Luo X, Cheng C, Tan Z, Li N, Tang M, Yang L, et al. Emerging roles of lipid metabolism in cancer metastasis. Molecular cancer. 2017;16(1):76. 


\section{Chapter 5}

60. Ma C, Xie J, Luo C, Yin H, Li R, Wang X, et al. OxLDL promotes lymphangiogenesis and lymphatic metastasis in gastric cancer by upregulating VEGFC expression and secretion. International journal of oncology. 2019;54(2):572-84.

61. Pascual G, Avgustinova A, Mejetta S, Martin M, Castellanos A, Attolini CS, et al. Targeting metastasis-initiating cells through the fatty acid receptor CD36. Nature. 2017;541(7635):41-5.

62. Bocci F, Jolly MK, Nelson Onuchic J. A biophysical model of Epithelial-Mesenchymal Transition uncovers the frequency and size distribution of Circulating Tumor Cell clusters across cancer types2019.

63. Morandi A, Taddei ML, Chiarugi P, Giannoni E. Targeting the Metabolic Reprogramming That Controls Epithelial-to-Mesenchymal Transition in Aggressive Tumors. Frontiers in oncology. 2017;7:40-.

64. Nath A, Li I, Roberts LR, Chan C. Elevated free fatty acid uptake via CD36 promotes epithelialmesenchymal transition in hepatocellular carcinoma. Sci Rep. 2015;5:14752.

65. Park YM, Drazba JA, Vasanji A, Egelhoff T, Febbraio M, Silverstein RL. Oxidized LDL/CD36 interaction induces loss of cell polarity and inhibits macrophage locomotion. Mol Biol Cell. 2012;23(16):3057-68.

66. Gonzalez-Chavarria I, Fernandez E, Gutierrez N, Gonzalez-Horta EE, Sandoval F, Cifuentes $\mathrm{P}$, et al. LOX-1 activation by oxLDL triggers an epithelial mesenchymal transition and promotes tumorigenic potential in prostate cancer cells. Cancer letters. 2018;414:34-43.

67. Gugnoni M, Sancisi V, Manzotti G, Gandolfi G, Ciarrocchi A. Autophagy and epithelialmesenchymal transition: an intricate interplay in cancer. Cell Death Dis. 2016;7(12):e2520-e.

68. Fan Q, Yang L, Zhang X, Ma Y, Li Y, Dong L, et al. Autophagy promotes metastasis and glycolysis by upregulating MCT1 expression and Wnt/beta-catenin signaling pathway activation in hepatocellular carcinoma cells. Journal of experimental \& clinical cancer research : CR. 2018;37(1):9.

69. Vera-Ramirez L, Vodnala SK, Nini R, Hunter KW, Green JE. Autophagy promotes the survival of dormant breast cancer cells and metastatic tumour recurrence. Nature Communications. 2018;9(1):1944.

70. Amaravadi R, Kimmelman AC, White E. Recent insights into the function of autophagy in cancer. Genes \& development. 2016;30(17):1913-30. 


\section{Chapter 5}

71. Qin W, Li C, Zheng W, Guo Q, Zhang Y, Kang M, et al. Inhibition of autophagy promotes metastasis and glycolysis by inducing ROS in gastric cancer cells. Oncotarget. 2015;6(37):39839-54.

72. Peng YF, Shi YH, Ding ZB, Ke AW, Gu CY, Hui B, et al. Autophagy inhibition suppresses pulmonary metastasis of $\mathrm{HCC}$ in mice via impairing anoikis resistance and colonization of HCC cells. Autophagy. 2013;9(12):2056-68.

73. Zhang Z, Pan X, Yang S, Ma A, Wang K, Wang Y, et al. miR-155 Promotes ox-LDL-Induced Autophagy in Human Umbilical Vein Endothelial Cells. Mediators of Inflammation. 2017;2017:7.

74. Yin S, Yang S, Pan X, Ma A, Ma J, Pei H, et al. MicroRNA-155 promotes ox-LDL-induced autophagy in human umbilical vein endothelial cells by targeting the PI3K/Akt/mTOR pathway. Mol Med Rep. 2018;18(3):2798-806.

75. Benitah S. Metastatic-initiating cells and lipid metabolism2017. 110-4 p.

76. Guillaumond F, Bidaut G, Ouaissi M, Servais S, Gouirand V, Olivares O, et al. Cholesterol uptake disruption, in association with chemotherapy, is a promising combined metabolic therapy for pancreatic adenocarcinoma. Proceedings of the National Academy of Sciences of the United States of America. 2015;112(8):2473-8.

77. Ahmadi Y, Karimian R, Panahi Y. Effects of statins on the chemoresistance-The antagonistic drug-drug interactions versus the anti-cancer effects. Biomedicine \& pharmacotherapy $=$ Biomedecine \& pharmacotherapie. 2018;108:1856-65.

78. Ma X, Bi E, Lu Y, Su P, Huang C, Liu L, et al. Cholesterol Induces CD8(+) T Cell Exhaustion in the Tumor Microenvironment. Cell metabolism. 2019.

79. Chen L, Jiang K, Jiang H, Wei P. miR-155 mediates drug resistance in osteosarcoma cells via inducing autophagy. Experimental and therapeutic medicine. 2014;8(2):527-32.

80. Kim TT, Dyck JR. The role of CD36 in the regulation of myocardial lipid metabolism. Biochimica et biophysica acta. 2016;1861(10):1450-60.

81. Umbarawan Y, Syamsunarno MRAA, Koitabashi N, Obinata H, Yamaguchi A, Hanaoka H, et al. Myocardial fatty acid uptake through CD36 is indispensable for sufficient bioenergetic metabolism to prevent progression of pressure overload-induced heart failure. Scientific Reports. 2018;8(1):12035.

82. Jiang Z-S, Sun Y-Z, Wang S-M, Ruan J-S. Epithelial-mesenchymal transition: potential regulator of $\mathrm{ABC}$ transporters in tumor progression. J Cancer. 2017;8(12):2319-27. 


\section{Chapter 5}

83. Sharom FJ. ABC multidrug transporters: structure, function and role in chemoresistance. Pharmacogenomics. 2008;9(1):105-27.

84. Begicevic R-R, Falasca M. ABC Transporters in Cancer Stem Cells: Beyond Chemoresistance. Int J Mol Sci. 2017;18(11):2362.

85. Zhao Q, Li Y, Tan BB, Fan LQ, Yang PG, Tian Y. HIF-1alpha Induces Multidrug Resistance in Gastric Cancer Cells by Inducing MiR-27a. PloS one. 2015;10(8):e0132746.

86. Shatrov VA, Sumbayev VV, Zhou J, Brune B. Oxidized low-density lipoprotein (oxLDL) triggers hypoxia-inducible factor-1alpha (HIF-1alpha) accumulation via redox-dependent mechanisms. Blood. 2003;101(12):4847-9.

87. Condamine T, Dominguez GA, Youn J-I, Kossenkov AV, Mony S, Alicea-Torres K, et al. Lectin-type oxidized LDL receptor-1 distinguishes population of human polymorphonuclear myeloid-derived suppressor cells in cancer patients. Sci Immunol. 2016;1(2):aaf8943. 


\section{Chapter 6}

OxLDL as an inducer of a metabolic shift in cancer cells 


\section{Chapter 6}

Chapter 6 OxLDL as an inducer of a metabolic shift in cancer cells

Albert V. Bitorina*, Yvonne Oligschlaeger*, Lingling Ding, Tulasi Yadati, Tom Houben, Rianne D.W. Vaes, Steven W.M. Olde Damink, Jan Theys" ${ }^{\#}$ Ronit Shiri-Sverdlov\#

*Contributed equally

Submitted 


\title{
Chapter 6
}

\begin{abstract}
Recent evidence established a link between disturbed lipid metabolism and increased risk for cancer. One of the most prominent features related to disturbed lipid metabolism is an increased production of oxidized low-density-lipoproteins (oxLDL), which results from elevated oxidative stress. OxLDL is known to have detrimental effects on healthy cells and plays a primary role in diseases related to the metabolic syndrome. Nevertheless, so far, the exact role of oxLDL in cancer cell metabolism is not yet known. To examine changes in metabolic profile induced by oxLDL, KLM-1 cells were treated with oxLDL in a dose- $(25$ or $50 \mu \mathrm{g} / \mathrm{ml})$ and/or time-dependent ( $4 \mathrm{hr}$ or $8 \mathrm{hr}$ ) manner and the impact of oxLDL on oxygen consumption rates (OCR) as well as extracellular acidification rates (ECAR) was analyzed using Seahorse technology. Subsequently, to establish the link between oxLDL and glycolysis, stabilization of the master regulator hypoxia-inducible factor 1-alpha (HIF-1 $\alpha$ ) was measured by means of Western blot. Furthermore, autophagic responses were assessed by measuring protein levels of the autophagosomal marker LC3B-II. Finally, the therapeutic potential of natural anti-oxLDL IgM antibodies in reversing these effects was tested. Incubation of KLM-1 cells with oxLDL shifted the energy balance towards a more glycolytic phenotype, which is an important hallmark of cancer cells. These data were supported by measurement of increased oxLDL-mediated HIF-1 $\alpha$ stabilization. In line, oxLDL incubation also increased the levels of LC3B-II, suggesting an elevated autophagic response. Importantly, antibodies against oxLDL were able to reverse these oxLDL-mediated metabolic effects. Our data provides a novel proof-of-concept that oxLDL induces a shift in energy balance. These data not only support a role for oxLDL in the progression of cancer but also suggest the possibility of targeting oxLDL as a therapeutic option in cancer.
\end{abstract}




\section{Chapter 6}

\section{Introduction}

Cancer is the second leading cause of deaths worldwide, which currently accounts for nearly ten million deaths (1). In particular, overweight and obesity, due to excess energy intake and decreased physical activity, have reached pandemic levels worldwide (2) and recent clinical data suggests that individuals with obesity are at an increased risk of developing several types of cancer (3-5).

Mechanisms that are known to link obesity with increased cancer burden, i.e., cancer development, progression and decreased treatment response (6), include adipose tissue dysfunction, increased circulating levels of hormones, perturbed lipid metabolism and low-grade chronic inflammation (7). Chronic inflammation is strongly associated with increased secretion of cytokines in the circulation, which can lead to cancer-related cellular processes such as cell adhesion, chemotaxis, migration and angiogenesis (8). Moreover, excess nutrient intake has been shown to increase the expression of multiple growth factors and hormones $(9,10)$ and is known to induce changes in gut microbiota $(11)$, thereby influencing oncogenesis and tumor progression.

One of the most prominent features related to excess nutrient intake and dysregulated lipid metabolism is the increased production of oxidized cholesterol-rich low-density lipoprotein (oxLDL), which results from elevated oxidative stress. Increased levels of oxLDL is known to have detrimental effects on healthy cells and plays a primary responsible role in metabolic syndrome (MetS)-related diseases such as atherosclerosis (12) and non-alcoholic steatohepatitis (NASH) (13). Strikingly, increasing evidence also points towards a correlation between circulating levels of oxLDL and the development of cancer (14), suggesting that oxLDL is a potent pro-oncogenic factor. Nevertheless, the exact metabolic role of oxLDL in cancer is not well-understood.

In the present study, we hypothesized that oxLDL induces a metabolic shift in cancer. We demonstrate for the first time that incubating KLM-1 pancreatic cancer cells with oxLDL shifts the energy balance towards a more glycolytic phenotype, which is supported by oxLDL-induced stabilization of hypoxiainducible factor 1-alpha (HIF-1 $\alpha$ ), a master regulator of glycolysis. In line with these data, we also show that oxLDL, likely due to increased autophagic activity, increases the expression of the autophagosomal marker LC3B-II. Essentially, the addition of EO6 IgM antibodies against oxLDL were able to reverse the oxLDL-mediated metabolic effects.

Altogether, our data provide a proof-of-concept that oxLDL induces a metabolic shift, which is involved in cancer progression. In addition, our data also supports the opportunity of targeting oxLDL as a novel therapeutic option in cancer. 


\section{Chapter 6}

\section{Materials and methods}

\section{Cell culture}

Human pancreatic cancer cell line KLM-1 (obtained from RIKEN BioResource Center) was cultured in high glucose (4.5 g/L) Dulbecco's Modified Eagle's Medium, supplemented with $10 \%$ (v/v) fetal bovine serum (USDA Approved, PAA, Pasching, Austria). Cells were maintained at $37^{\circ} \mathrm{C}, 5 \% \mathrm{CO} 2$ in a humidified incubator.

\section{Energy metabolism assay}

KLM-1 cells were grown in DMEM + 10\% FBS and seeded into 96XF plates @ 24000 cells/well and left to attach overnight at $37^{\circ} \mathrm{C}$. Metabolic profiles were generated by replacing the growth medium with assay medium $1 \mathrm{hr}$ before measurement according to the manufacturer guidelines (15). OxLDL (Alfa Aesar) was titrated to $25 \mu \mathrm{g} / \mathrm{ml}$ over $60 \mathrm{~min}$. Oxygen consumption and extracellular acidification rates were monitored using a Seahorse Bioscience XF96 Extracellular Flux Analyzer. Values were corrected versus baseline as well as for total protein content in each well. Total protein concentration was measured using a Pierce ${ }^{\circledR}$ (Waltham, MA, USA). Data were obtained and depicted using Wave 2.6.0.31 software (Agilent).

\section{Western blot}

In 3 independent experiments, KLM-1 cells were grown in DMEM + 10\% FBS and seeded into 6 well plates at 500,000 cells/well and left to attach overnight at $37^{\circ} \mathrm{C}$. Subsequently, cells were treated with oxLDL ( 25 or $50 \mu \mathrm{g} / \mathrm{ml}$; Tebu-Bio) for $4 \mathrm{hr}$ or $8 \mathrm{hr}$. Alternatively, cells were either treated with oxLDL alone $(50 \mu \mathrm{g} / \mathrm{ml})$, oxLDL $(50 \mu \mathrm{g} / \mathrm{ml})+$ chloroquine $(20 \mu \mathrm{M})$, oxLDL $(50 \mu \mathrm{g} / \mathrm{ml})+$ EO6 $(14 \mu \mathrm{g} / \mathrm{ml}$; Avanti) or oxLDL $(50 \mu \mathrm{g} / \mathrm{ml})+$ chloroquine $(20 \mu \mathrm{M})+$ EO6 $(14 \mu \mathrm{g} / \mathrm{ml})$. Subsequently, cells were washed with cold PBS and lysed with cold RIPA buffer (50 mM Tris-HCL pH 7.5, $150 \mathrm{mM} \mathrm{NaCl,} \mathrm{0.5 \%} \mathrm{Sodium}$ deoxycholate, $1 \%$ Triton X-100, $0.1 \%$ SDS) supplemented with a mixture of protease and phosphatase inhibitors (Complete and PhosStop; Roche). Lysates were centrifuged at 16,000 xg for 15 min, after which the supernatant was transferred to a new eppendorf and stored at $-20^{\circ} \mathrm{C}$. The total protein concentration was measured using a Pierce ${ }^{\circledR}$ (Waltham, MA, USA) BCA protein assay. Equal amounts of protein were loaded on the gel. After SDS/PAGE, proteins were transferred to nitrocellulose membrane (Bio-Rad), which was blocked with 5\% non-fat dry milk for $1 \mathrm{~h}$ at room temperature. For detection, the membrane was incubated with an antibody against HIF-1 $\alpha$ (1:2000 dilution; BD Transduction Laboratories; Catalogue \#610959), $\beta$-Actin (1:2000 dilution; Cell Signaling, \#4967) and 


\section{Chapter 6}

LC3B (1:1000 dillution; Cell Signaling, \#2775) overnight at $4^{\circ} \mathrm{C}$ followed by $1 \mathrm{~h}$ of incubation with donkey-anti-rabbit or rat-anti-mouse detection antibody, respectively, at room temperature (1:2000 Jackson Laboratories, Bar Harbor, ME, USA). Signal was detected with the Biorad ChemiDoc ${ }^{\mathrm{TM}}$ XRS+System by enhanced chemoluminescence. Blots were subsequently quantified using ImageJ software (Ver 1.52R). HIF-1 $\alpha$, LC3B-I and LC3B-II levels were first corrected for loading by $\beta$-Actin, after which these levels were normalized to medium control or OxLDL respectively. Fold-changes are represented in bar graphs that were generated using GraphPad 6 software.

\section{Statistical analysis}

Data were statistically analyzed by performing two-tailed nonpaired t tests using GraphPad Prism (version 6 for Windows; GraphPad Software, San Diego, CA) for comparing differences in ECAR\% between control and oxLDL-treated KLM-1 cells. One-way ANOVA with multiple comparisons Tukey pos-hoc test was used to compare differences of HIF-1 $\alpha$ or LC3B-II between control and oxLDL treated KLM-1 cells pooled from 3 independent experiments. The results were visualized and expressed as the mean \pm SEM and considered significant at $\mathrm{p} \leq 0.05, *$ and $* *$ indicate $\mathrm{p} \leq 0.01$ respectively. 


\section{Chapter 6}

\section{Results}

\section{OxLDL increases glycolysis in KLM-1 cells}

Oxygen consumption rate (OCR) is routinely used as an indicator of mitochondrial oxidative phosphorylation, whereas extracellular acidification rates (ECAR) reflect the state of lactate secretion into the medium, which is strongly linked to the levels of glycolysis in the cell. In order to investigate the effects of oxLDL on cellular energy balance in cancer cells, we titrated $25 \mu \mathrm{g} / \mathrm{ml}$ of oxLDL, under normoxic conditions, to the pancreatic tumor cell line KLM-1 and measured real-time basal changes in OCR and ECAR over the course of $60 \mathrm{~min}$. Whereas no effect was observed on OCR rates, our data showed that incubation with oxLDL shifted the energy balance towards a more glycolytic phenotype, as shown by a $20 \%$ increase in ECAR compared to control cells $(\mathbf{F i g . ~ 1 A , B})$.

A

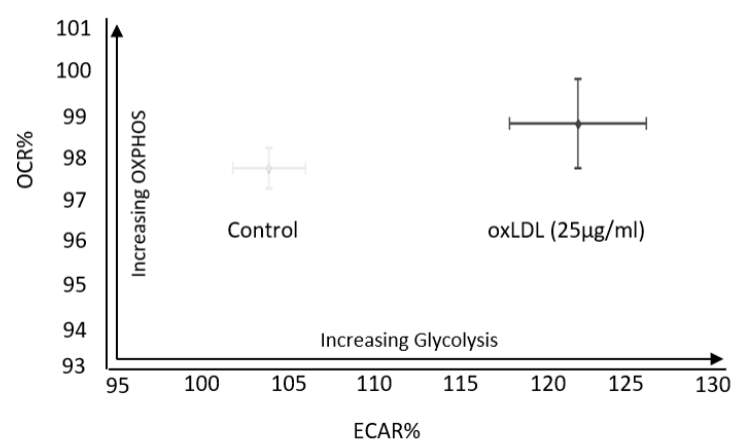

B

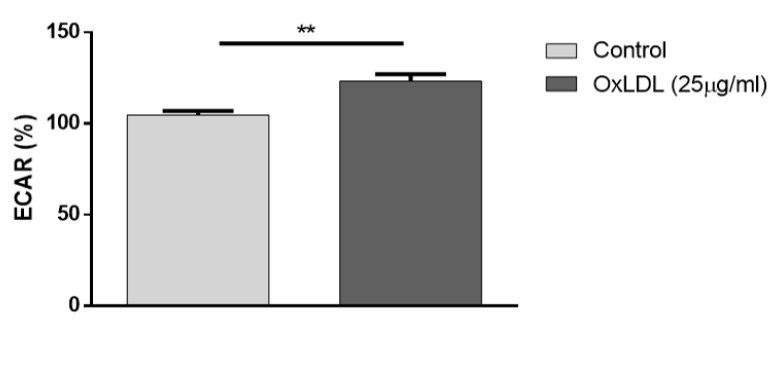

Figure 1: OxLDL shifts energy balance to a glycolytic profile. KLM-1 pancreatic cancer cells were titrated with oxLDL $(25 \mu \mathrm{g} / \mathrm{ml})$. (A) Representative energy metabolism diagram of basal OCR vs. ECAR. (B) Difference in ECAR\% between control and oxLDL-treated cells. ${ }^{* *} p<0.01$

\section{OxLDL stabilizes HIF-1 $\alpha$ protein}

HIF- $1 \alpha$ is a master regulator of glycolysis and high levels of the stabilized protein is maintained in cells with high glycolytic rates. In order to determine the effect of oxLDL on HIF-1 $\alpha$ protein stabilization, KLM-1 cells were incubated in the absence or presence of different concentrations of oxLDL ( 25 or $50 \mu \mathrm{g} / \mathrm{ml}$, respectively) for $4 \mathrm{hr}$ or $8 \mathrm{hr}$. Our results demonstrated that protein levels of HIF-1 $\alpha$ increased in response to 50 but not $25 \mu \mathrm{g} / \mathrm{ml}$ oxLDL treatment compared to control ( $\mathrm{p}=0.056)$ (Fig. $\mathbf{2 A}, \mathbf{B}$; Supplemental Fig1). Furthermore, significant differences in HIF-1 $\alpha$ protein were observed after incubation of $8 \mathrm{hr}$ compared to $4 \mathrm{hr}$, suggesting that the oxLDL-mediated increase in ECAR resulted from HIF- $1 \alpha$ protein stabilization in a dose- and time-dependent manner. 
A

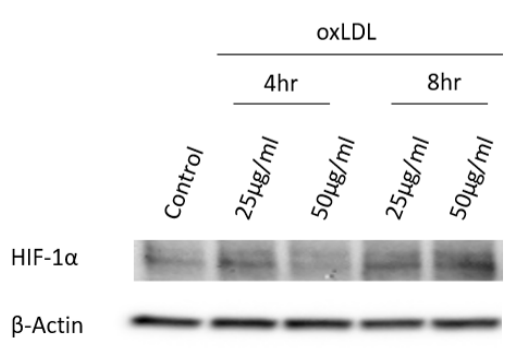

B

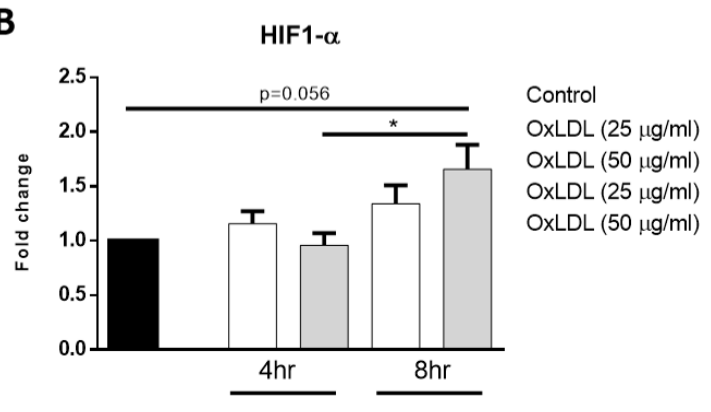

Figure 2: OxLDL stabilizes HIF-1 $\alpha$ protein levels. KLM-1 cells were treated with oxLDL ( 25 or $50 \mu \mathrm{g} / \mathrm{ml}$ ) for $4 \mathrm{hr}$ or 8hr. (A) Representative Western blot of HIF-1 $\alpha$ protein levels, (B) Quantification of HIF-1 $\alpha$ protein.

\section{OxLDL increases autophagosomal marker LC3B-II}

In addition to its involvement in glycolysis, HIF-1 $\alpha$ can also modulate autophagy in order to secure additional energy during metabolic stress responses such as hypoxia. It has been shown that the cytosolic protein LC3B-I is converted to autophagosomal marker LC3B-II during the fusion of endosomes with lysosomes, a process known as autophagosome formation (16). In order to assess whether autophagic activity is induced in response to oxLDL, we treated KLM-1 cells with or without oxLDL ( 25 and $50 \mu \mathrm{g} / \mathrm{ml}$, respectively) over a time course of $4 \mathrm{hr}$ and $8 \mathrm{hr}$. Subsequently, protein levels of LC3B-II were determined with Western blot (Fig. 3A; Supplemental Fig1) and quantitative data analysis showed that incubation with oxLDL resulted in a 4-fold increase of LC3B-II protein expression after 8hr (p=0.07) (Fig. 3B; Supplemental Fig1).

A

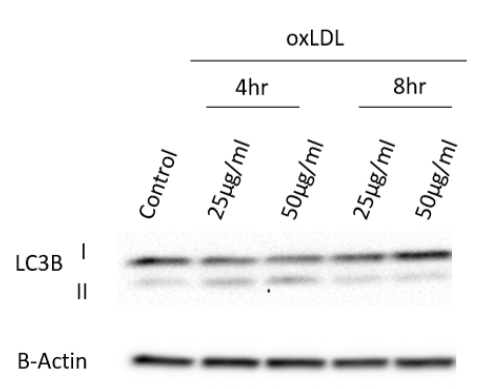

B

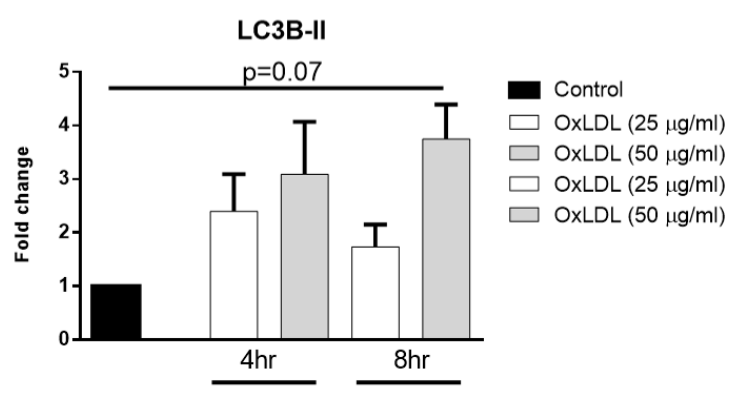

Figure 3 OxLDL increases LC3B-II proteins levels. KLM-1 cells were treated with oxLDL ( 25 or $50 \mu \mathrm{g} / \mathrm{ml}$ ) for $4 \mathrm{hr}$ or 8hr. (A) Representative Western blot of LC3B-I and LC3B-II protein levels, (B) Quantification of LC3B-II protein. 


\section{Chapter 6}

\section{EO6 reverses oxLDL-induced metabolic changes}

Given the fact that natural IgM antibodies against oxLDL have been shown to ameliorate the adverse effects of oxLDL in several metabolic diseases, including NASH (17) and atherosclerosis (18), we assessed whether EO6 monoclonal antibodies against oxLDL were able to counteract the metabolic effects of oxLDL on HIF-1 $\alpha$ and LC3B by treating KLM-1 cells for 8hr with oxLDL (50 $\mu \mathrm{g} / \mathrm{ml})$ in the presence or absence of EO6 $(14 \mu \mathrm{g} / \mathrm{ml})$. Additionally, cells were also treated with chloroquine $(\mathrm{Cq}$; $20 \mu \mathrm{M}$ ) in order to ensure that any observed differences in LC3B-II were due to increased conversion of LC3B-I rather than differences in breakdown of LC3B-II. Chloroquine is a lysosomotropic weak base that diffuses into the lysosome, where it gets trapped, thereby changing the lysosomal $\mathrm{pH}$ and subsequently inhibiting autophagic degradation of proteins including LC3B-II (19). Our results showed that treatment with EO6 significantly reversed oxLDL-mediated increases in HIF-1 $\alpha$ protein, in the presence of chloroquine (Fig. 4A,B). Similarly, treatment with EO6 also significantly reversed oxLDLmediated increases in LC3B-II protein, in the presence of chloroquine. (Fig. 4A, C; Supplemental Fig1).

A

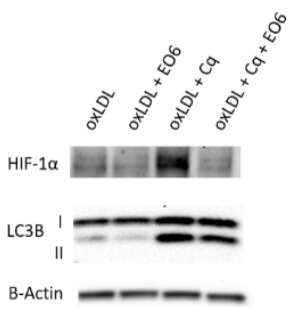

B

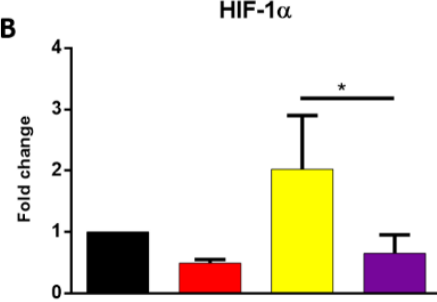

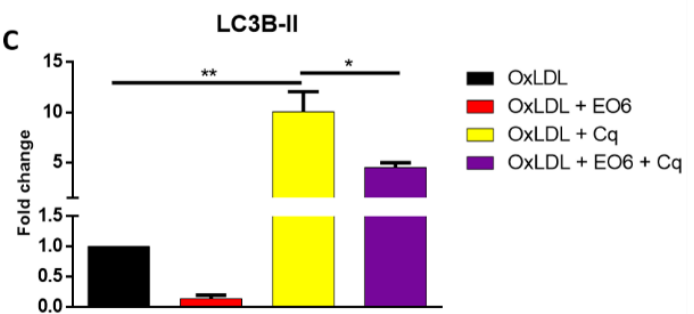

Figure 4: EO6 reverses oxLDL-mediated effects on HIF-1 $\alpha$ and LC3B-II. KLM-1 cells were treated with oxLDL $(50 \mu \mathrm{g} / \mathrm{ml})$ in the presence or absence of chloroquine $(\mathrm{Cq} ; 20 \mu \mathrm{M})$ and EO6 antibodies against oxLDL $(14 \mu \mathrm{g} / \mathrm{ml})$ for $8 \mathrm{hr}$. (A) Representative Western blot of HIF-1 $\alpha$ and LC3B-I and LC3B-II protein levels, (B) Quantification of HIF-1 $\alpha$ and (C) LC3B-II protein. 


\section{Chapter 6}

\section{Discussion}

Metabolic reprogramming, referred to as an important cellular adaptation mechanism, is unequivocally considered a hallmark of cancer (20). Many studies have focused on gaining better insight into the complex nature of cancer cell metabolism both in-vitro $(21,22)$ and in-vivo $(23)$, and recently, the impact of abnormal lipid metabolism in modulating cancer pathophysiology has received increasing interest (24). In the current study, we provide novel insights into the role of oxLDL in regulating cancer cell metabolism.

Due to their high proliferation rate, cancer cells show a strong avidity for lipids and cholesterol, which they retrieve either from increased uptake of dietary lipids and/or cholesterol, or from enhanced lipogenesis and cholesterol synthesis $(25,26)$. One of the most prominent features associated with increased dietary lipids, and hence dyslipidemia, is low-density lipoprotein (LDL) oxidation. Oxidative modifications of LDL can occur as a result of elevated oxidative stress, a metabolic derangement known to be common in cancer and MetS-related diseases.

Previously, it has been shown that increased levels of oxLDL has detrimental effects on healthy metabolic tissues, including the cardiovascular system (27) and the liver (28). For instance, oxLDL impaired mitochondrial respiration in porcine aortic endothelial cells due to reduced activities of mitochondrial complexes (29), likely inducing a metabolic shift. Furthermore, overloading macrophages with oxLDL triggered a metabolic switch that resulted in high levels of HIF1- $\alpha(30,31)$, which is a master regulator of metabolism that is constitutively expressed in cells. Normally, in the presence of oxygen, HIF-1 $\alpha$ is destabilized and targeted for proteosomal degradation (32). However, under abnormal conditions such as hypoxia, which is a common feature of cancer, HIF-1 $\alpha$ is being stabilized, thereby mediating a switch towards glycolytic metabolism (33).

Given the fact that oxLDL stimulates the proliferation of a variety of cell types, including macrophages, endothelial and smooth muscle cells (34-36), oxLDL has been suggested to have pro-oncogenic properties. Indeed, in the context of cancer, it has been reported that increased accumulation of oxLDL, which is related to hyperlipidemia, is highly associated with the development of several types of cancer, including colon, breast and ovarian cancer $(14,37,38)$. Nevertheless, the exact mechanism of oxLDL on cancer cell metabolism has not yet been fully understood.

In the present study, we demonstrate for the first time that incubating KLM-1 pancreatic cancer cells with oxLDL, under normoxic conditions, leads to HIF-1 $\alpha$ stabilization. These data are in line with others showing that oxLDL induced HIF-1 $\alpha$ protein accumulation in human macrophages $(30,31)$. Furthermore, these data also support the observed increase in basal ECAR levels, pointing towards oxLDL as an inducer of a metabolic switch towards glycolysis in cancer cells. Given that cancer cells, 


\section{Chapter 6}

even under non-hypoxic conditions, are capable of inducing HIF-1 $\alpha$ and can actively shift their energy production from mitochondrial to glycolytic sources (39), our data suggest that treatment of oxLDL mimics a 'Warburg'-like effect in KLM-1 pancreatic cancer cells.

One of the most conserved physiological processes present in eukaryotes is autophagy, a catabolic pathway that is known to involve lysosomal degradation of organelles and cytoplasmic contents that are recycled for sustaining cellular energy requirements (40). It is known that under some circumstances, autophagy may be utilized as a cellular 'suicide' mechanism, thereby inducing cell death (41). Recent studies also discovered several protective functions for autophagy, including the regulation of intracellular lipid stores and a role in immunity, cell death and inflammation $(42,43)$. For instance, in the context of MetS, we demonstrated that oxLDL disturbs lysosomal function (28) and induces autophagy (44), which was correlated with hepatic inflammation, whereas others found that elevated levels of oxLDL enabled the selection and survival of cancer cells due to its involvement in protective autophagy (45), suggesting a multifaceted role of oxLDL.

Previous studies showed that metabolic derangements, such as hypoxia, can cause a remarkable upregulation of HIF-1 $\alpha$ (46), triggering cellular autophagy and glucose uptake in mouse granulosa tumor cells (47). In line with these studies, we showed that oxLDL treatment resulted in an increase in the autophagosomal marker LC3B-II, which is a central protein in the regulation of autophagy (48). Hence, our data suggest that oxLDL stabilizes HIF-1 $\alpha$, thereby activating the autophagic pathway, as shown by the concomitant increase in LC3B-II protein. Based on other studies $(49,50)$, it is very likely that these changes are responsible for the oxLDL-induced metabolic switch towards glycolysis observed in KLM-1 pancreatic cancer cells, which is crucial for cell survival.

Given that oxLDL is involved in many aspects of metabolic diseases, it has become a promising target in chronic inflammatory diseases, in which lipid metabolism is disturbed. Exercise training $(51,52)$, lowering dietary fat intake (53) as well as antioxidant $(54,55)$, statin (56) and fenofibrate therapies (57) are therapeutic strategies that have been involved in lowering oxLDL levels. Relevantly, these strategies have also been shown to reduce cancer risk (58) or exhibit anticancer properties (59-61), of which some therapeutic modalities have been already used in clinical trials. In the context of MetS, several studies have demonstrated that circulating levels of oxLDL can also be effectively targeted in a more direct manner without inducing side effects, as unfortunately often seen with adjuvant therapies (62). Recent evidence indicates that immunizing hyperlipidemic mice with Streptococcus pneumoniae is an effective way of triggering a natural immune response (i.e., inducing an increase in monoclonal IgM autoantibodies) against oxLDL, thereby reducing atherosclerotic lesions (18). Similarly, in the context of NASH, we previously demonstrated that specific targeting of oxLDL by means of pneumococcal immunization also reduced hepatic inflammation (17), suggesting that the scavenging of oxLDL by anti-oxLDL autoantibodies might be a potent therapeutic strategy in cancer treatment. Obviously, 


\section{Chapter 6}

chronic inflammation is also strongly associated with malignant diseases $(63,64)$ and tumor-associated macrophages are known to represent the major inflammatory cell population in tumors. Hence, several studies explored the effects of anti-inflammatory strategies, such as nonsteroidal anti-inflammatory drugs, and showed their ability to prevent early onset of cancer (65) and reduce the risk of cancer (66), thus underscoring the significance of inflammation during neoplastic progression.

In the current study, we explored the effects of natural IgM antibodies against oxLDL (referred to as EO6) in the context of cancer cell metabolism. Our results showed that EO6, by scavenging oxLDL, is capable of reducing the ability of oxLDL to stabilize HIF-1 $\alpha$ and the subsequent increase in autophagy. One way in which cancer cells become resilient or resistant, and thus enhance their capability to proliferate or metastasize, is by metabolically switching to glycolytic profiles and inducing cellular protective autophagy. Hence, several strategies, focused on reorienting cancer cell metabolism from glycolysis to oxidative phosphorylation, have demonstrated a beneficial decrease in cell survival, invasiveness and tumor growth. For instance, Poteet et al. showed that methylene blue was capable of increasing oxygen consumption while reducing lactate production, thereby reversing the glycolytic profile and decreasing proliferation of glioblastoma cell (67). Another study showed that resveratrol increased ATP production and thus the oxidative capacity, while decreasing glycolysis, as shown by a reduction in the pentose phosphate pathway in colon cancer cells (68). Moreover, they also demonstrated that resveratrol was able to modify the lipodomic profile of these cells, which is likely important for its metabolic effects on cancer energy metabolism.

\section{Conclusions}

In the present study, we demonstrate for the first time that in addition to the known beneficial metabolic effects of the tumor microenvironment, oxLDL has a direct effect on pancreatic cancer cells by promoting a metabolic shift towards a more glycolytic phenotype. Furthermore, our data revealed that the addition of EO6 IgM antibodies against oxLDL were able to reverse these oxLDL-mediated metabolic effects. While there are several studies looking into the effects of oxLDLin other types of cancer, little is known about its effect in pancreatic cancer. As oxLDl is likely to also play a role in pancreatic cancer, future studies should therefore look into the effect of oxLDL in pancreatic cancer and evaluate the potential of targeting oxLDL as a novel therapeutic option in cancer. 


\section{Chapter 6}

\section{References}

1. Cabukusta B and Neefjes J: Mechanisms of lysosomal positioning and movement. Traffic 19: 761-769, 2018.

2. Friedrich MJ: Global Obesity Epidemic Worsening. JAMA 318: 603, 2017.

3. Doerstling SS, O'Flanagan $\mathrm{CH}$ and Hursting SD: Obesity and Cancer Metabolism: A Perspective on Interacting Tumor-Intrinsic and Extrinsic Factors. Front Oncol 7: 216, 2017.

4. Chen Y, Wang X, Wang J, Yan Z and Luo J: Excess body weight and the risk of primary liver cancer: an updated meta-analysis of prospective studies. Eur J Cancer 48: 2137-2145, 2012.

5. Genkinger JM, Spiegelman D, Anderson KE, et al.: A pooled analysis of 14 cohort studies of anthropometric factors and pancreatic cancer risk. Int J Cancer 129: 1708-1717, 2011.

6. Esposito K, Chiodini P, Colao A, Lenzi A and Giugliano D: Metabolic syndrome and risk of cancer: a systematic review and meta-analysis. Diabetes Care 35: 2402-2411, 2012.

7. Micucci C, Valli D, Matacchione G and Catalano A: Current perspectives between metabolic syndrome and cancer. Oncotarget 7: 38959-38972, 2016.

8. Landskron G, De la Fuente M, Thuwajit P, Thuwajit $\mathrm{C}$ and Hermoso MA: Chronic inflammation and cytokines in the tumor microenvironment. J Immunol Res 2014: 149185, 2014.

9. Brahmkhatri VP, Prasanna $\mathrm{C}$ and Atreya HS: Insulin-like growth factor system in cancer: novel targeted therapies. Biomed Res Int 2015: 538019, 2015.

10. Capper CP, Rae JM and Auchus RJ: The Metabolism, Analysis, and Targeting of Steroid Hormones in Breast and Prostate Cancer. Horm Cancer 7: 149-164, 2016.

11. Zitvogel L, Galluzzi L, Viaud S, et al.: Cancer and the gut microbiota: an unexpected link. Sci Transl Med 7: 271ps271, 2015.

12. Di Pietro N, Formoso G and Pandolfi A: Physiology and pathophysiology of oxLDL uptake by vascular wall cells in atherosclerosis. Vascul Pharmacol 84: 1-7, 2016.

13. Walenbergh SM, Koek GH, Bieghs V and Shiri-Sverdlov R: Non-alcoholic steatohepatitis: the role of oxidized low-density lipoproteins. J Hepatol 58: 801-810, 2013. 


\section{Chapter 6}

14. Suzuki K, Ito Y, Wakai K, et al.: Serum oxidized low-density lipoprotein levels and risk of colorectal cancer: a case-control study nested in the Japan Collaborative Cohort Study. Cancer Epidemiol Biomarkers Prev 13: 1781-1787, 2004.

15. Nicholls DG, Darley-Usmar VM, Wu M, Jensen PB, Rogers GW and Ferrick DA: Bioenergetic profile experiment using C2C12 myoblast cells. J Vis Exp2010.

16. Kabeya Y, Mizushima N, Ueno T, et al.: LC3, a mammalian homologue of yeast Apg8p, is localized in autophagosome membranes after processing. EMBO J 19: 5720-5728, 2000.

17. Bieghs V, van Gorp PJ, Walenbergh SM, et al.: Specific immunization strategies against oxidized low-density lipoprotein: a novel way to reduce nonalcoholic steatohepatitis in mice. Hepatology 56: 894-903, 2012.

18. Binder CJ, Horkko S, Dewan A, et al.: Pneumococcal vaccination decreases atherosclerotic lesion formation: molecular mimicry between Streptococcus pneumoniae and oxidized LDL. Nat Med 9: 736-743, 2003.

19. Redmann M, Benavides GA, Berryhill TF, et al.: Inhibition of autophagy with bafilomycin and chloroquine decreases mitochondrial quality and bioenergetic function in primary neurons. Redox Biol 11: 73-81, 2017.

20. Hanahan D and Weinberg RA: Hallmarks of cancer: the next generation. Cell 144: 646-674, 2011.

21. Zhang Y and Yang JM: Altered energy metabolism in cancer: a unique opportunity for therapeutic intervention. Cancer Biol Ther 14: 81-89, 2013.

22. Feinberg T, Herbig J, Kohl I, et al.: Cancer metabolism: the volatile signature of glycolysis-in vitro model in lung cancer cells. J Breath Res 11: 016008, 2017.

23. Faubert B, Li KY, Cai L, et al.: Lactate Metabolism in Human Lung Tumors. Cell 171: 358371 e359, 2017.

24. Maan M, Peters JM, Dutta M and Patterson AD: Lipid metabolism and lipophagy in cancer. Biochem Biophys Res Commun 504: 582-589, 2018.

25. Swinnen JV, Brusselmans K and Verhoeven G: Increased lipogenesis in cancer cells: new players, novel targets. Curr Opin Clin Nutr Metab Care 9: 358-365, 2006.

26. Baenke F, Peck B, Miess H and Schulze A: Hooked on fat: the role of lipid synthesis in cancer metabolism and tumour development. Dis Model Mech 6: 1353-1363, 2013. 


\section{Chapter 6}

27. Galle J, Hansen-Hagge T, Wanner C and Seibold S: Impact of oxidized low density lipoprotein on vascular cells. Atherosclerosis 185: 219-226, 2006.

28. Bieghs V, Walenbergh SM, Hendrikx T, et al.: Trapping of oxidized LDL in lysosomes of Kupffer cells is a trigger for hepatic inflammation. Liver Int 33: 1056-1061, 2013.

29. Xie X, Chowdhury SR, Sangle G and Shen GX: Impact of diabetes-associated lipoproteins on oxygen consumption and mitochondrial enzymes in porcine aortic endothelial cells. Acta Biochim Pol 57: 393-398, 2010.

30. Shatrov VA, Sumbayev VV, Zhou J and Brune B: Oxidized low-density lipoprotein (oxLDL) triggers hypoxia-inducible factor-1alpha (HIF-1alpha) accumulation via redox-dependent mechanisms. Blood 101: 4847-4849, 2003.

31. Poitz DM, Augstein A, Weinert S, Braun-Dullaeus RC, Strasser RH and Schmeisser A: OxLDL and macrophage survival: essential and oxygen-independent involvement of the Hif-pathway. Basic Res Cardiol 106: 761-772, 2011.

32. Ivan M, Kondo K, Yang H, et al.: HIFalpha targeted for VHL-mediated destruction by proline hydroxylation: implications for O2 sensing. Science 292: 464-468, 2001.

33. Semenza GL: Regulation of metabolism by hypoxia-inducible factor 1. Cold Spring Harb Symp Quant Biol 76: 347-353, 2011.

34. Zettler ME, Prociuk MA, Austria JA, Massaeli H, Zhong G and Pierce GN: OxLDL stimulates cell proliferation through a general induction of cell cycle proteins. Am J Physiol Heart Circ Physiol 284: H644-653, 2003.

35. Seibold S, Schurle D, Heinloth A, Wolf G, Wagner M and Galle J: Oxidized LDL induces proliferation and hypertrophy in human umbilical vein endothelial cells via regulation of p27Kip1 expression: role of RhoA. J Am Soc Nephrol 15: 3026-3034, 2004.

36. Liu J, Ren Y, Kang L and Zhang L: Oxidized low-density lipoprotein increases the proliferation and migration of human coronary artery smooth muscle cells through the upregulation of osteopontin. Int J Mol Med 33: 1341-1347, 2014.

37. Delimaris I, Faviou E, Antonakos G, Stathopoulou E, Zachari A and Dionyssiou-Asteriou A: Oxidized LDL, serum oxidizability and serum lipid levels in patients with breast or ovarian cancer. Clin Biochem 40: 1129-1134, 2007.

38. Chen KC, Liao YC, Wang JY, Lin YC, Chen CH and Juo SH: Oxidized low-density lipoprotein is a common risk factor for cardiovascular diseases and gastroenterological cancers via epigenomical regulation of microRNA-210. Oncotarget 6: 24105-24118, 2015. 


\section{Chapter 6}

39. Koppenol WH, Bounds PL and Dang CV: Otto Warburg's contributions to current concepts of cancer metabolism. Nat Rev Cancer 11: 325-337, 2011.

40. Yorimitsu T and Klionsky DJ: Autophagy: molecular machinery for self-eating. Cell Death Differ 12 Suppl 2: 1542-1552, 2005.

41. Lin L and Baehrecke EH: Autophagy, cell death, and cancer. Mol Cell Oncol 2: e985913, 2015.

42. Levine B, Mizushima N and Virgin HW: Autophagy in immunity and inflammation. Nature 469: 323-335, 2011.

43. Singh R, Kaushik S, Wang Y, et al.: Autophagy regulates lipid metabolism. Nature 458: 1131$1135,2009$.

44. Hendrikx T, Bieghs V, Walenbergh SM, et al.: Macrophage specific caspase-1/11 deficiency protects against cholesterol crystallization and hepatic inflammation in hyperlipidemic mice. PLoS One 8: e78792, 2013.

45. Zabirnyk O, Liu W, Khalil S, Sharma A and Phang JM: Oxidized low-density lipoproteins upregulate proline oxidase to initiate ROS-dependent autophagy. Carcinogenesis 31: 446-454, 2010.

46. Schofield CJ and Ratcliffe PJ: Oxygen sensing by HIF hydroxylases. Nat Rev Mol Cell Biol 5: 343-354, 2004.

47. Zhou J, Li C, Yao W, et al.: Hypoxia-inducible factor-1alpha-dependent autophagy plays a role in glycolysis switch in mouse granulosa cells. Biol Reprod 99: 308-318, 2018.

48. Tanida I, Ueno T and Kominami E: LC3 and Autophagy. Methods Mol Biol 445: 77-88, 2008.

49. Papandreou I, Cairns RA, Fontana L, Lim AL and Denko NC: HIF-1 mediates adaptation to hypoxia by actively downregulating mitochondrial oxygen consumption. Cell Metab 3: 187197, 2006.

50. Denko NC: Hypoxia, HIF1 and glucose metabolism in the solid tumour. Nat Rev Cancer 8: 705-713, 2008.

51. Shern-Brewer R, Santanam N, Wetzstein C, White-Welkley J and Parthasarathy S: Exercise and cardiovascular disease: a new perspective. Arterioscler Thromb Vasc Biol 18: 1181-1187, 1998.

52. Takashima A, Ise T, Yagi S, et al.: Cardiac rehabilitation reduces serum levels of oxidized lowdensity lipoprotein. Circ J 78: 2682-2687, 2014. 


\section{Chapter 6}

53. Silaste ML, Rantala M, Alfthan G, et al.: Changes in dietary fat intake alter plasma levels of oxidized low-density lipoprotein and lipoprotein(a). Arterioscler Thromb Vasc Biol 24: 498$503,2004$.

54. Harris WS: The prevention of atherosclerosis with antioxidants. Clin Cardiol 15: 636-640, 1992.

55. Najafpour Boushehri S, Yusof RM, Nasir Mohammad Taib M, Mirzaei K, Yazdekhasti N and Akbarzadeh S: Effect of vitamin supplementation on serum oxidized low-density lipoprotein levels in male subjects with cardiovascular disease risk factors. Iran J Basic Med Sci 15: 958$964,2012$.

56. Singh U, Devaraj S, Jialal I and Siegel D: Comparison effect of atorvastatin (10 versus $80 \mathrm{mg}$ ) on biomarkers of inflammation and oxidative stress in subjects with metabolic syndrome. Am J Cardiol 102: 321-325, 2008.

57. Rosenson RS, Wolff DA, Huskin AL, Helenowski IB and Rademaker AW: Fenofibrate therapy ameliorates fasting and postprandial lipoproteinemia, oxidative stress, and the inflammatory response in subjects with hypertriglyceridemia and the metabolic syndrome. Diabetes Care 30: 1945-1951, 2007.

58. Prentice RL, Thomson CA, Caan B, et al.: Low-fat dietary pattern and cancer incidence in the Women's Health Initiative Dietary Modification Randomized Controlled Trial. J Natl Cancer Inst 99: 1534-1543, 2007.

59. Borgquist S, Bjarnadottir O, Kimbung S and Ahern TP: Statins: a role in breast cancer therapy? J Intern Med 284: 346-357, 2018.

60. Lian X, Gu J, Gao B, et al.: Fenofibrate inhibits mTOR-p70S6K signaling and simultaneously induces cell death in human prostate cancer cells. Biochem Biophys Res Commun 496: 70-75, 2018 .

61. Thyagarajan A and Sahu RP: Potential Contributions of Antioxidants to Cancer Therapy: Immunomodulation and Radiosensitization. Integr Cancer Ther 17: 210-216, 2018.

62. Shapiro CL and Recht A: Side effects of adjuvant treatment of breast cancer. N Engl J Med 344: 1997-2008, 2001.

63. Coussens LM and Werb Z: Inflammation and cancer. Nature 420: 860-867, 2002.

64. Multhoff G, Molls M and Radons J: Chronic inflammation in cancer development. Front Immunol 2: 98, 2011. 


\section{Chapter 6}

65. Baron JA and Sandler RS: Nonsteroidal anti-inflammatory drugs and cancer prevention. Annu Rev Med 51: 511-523, 2000.

66. Garcia-Rodriguez LA and Huerta-Alvarez C: Reduced risk of colorectal cancer among longterm users of aspirin and nonaspirin nonsteroidal antiinflammatory drugs. Epidemiology 12: 88-93, 2001.

67. Poteet E, Choudhury GR, Winters A, et al.: Reversing the Warburg effect as a treatment for glioblastoma. J Biol Chem 288: 9153-9164, 2013.

68. Saunier E, Antonio S, Regazzetti A, et al.: Resveratrol reverses the Warburg effect by targeting the pyruvate dehydrogenase complex in colon cancer cells. Sci Rep 7: 6945, 2017. 


\section{Chapter 6}

\section{Supplementary figures}

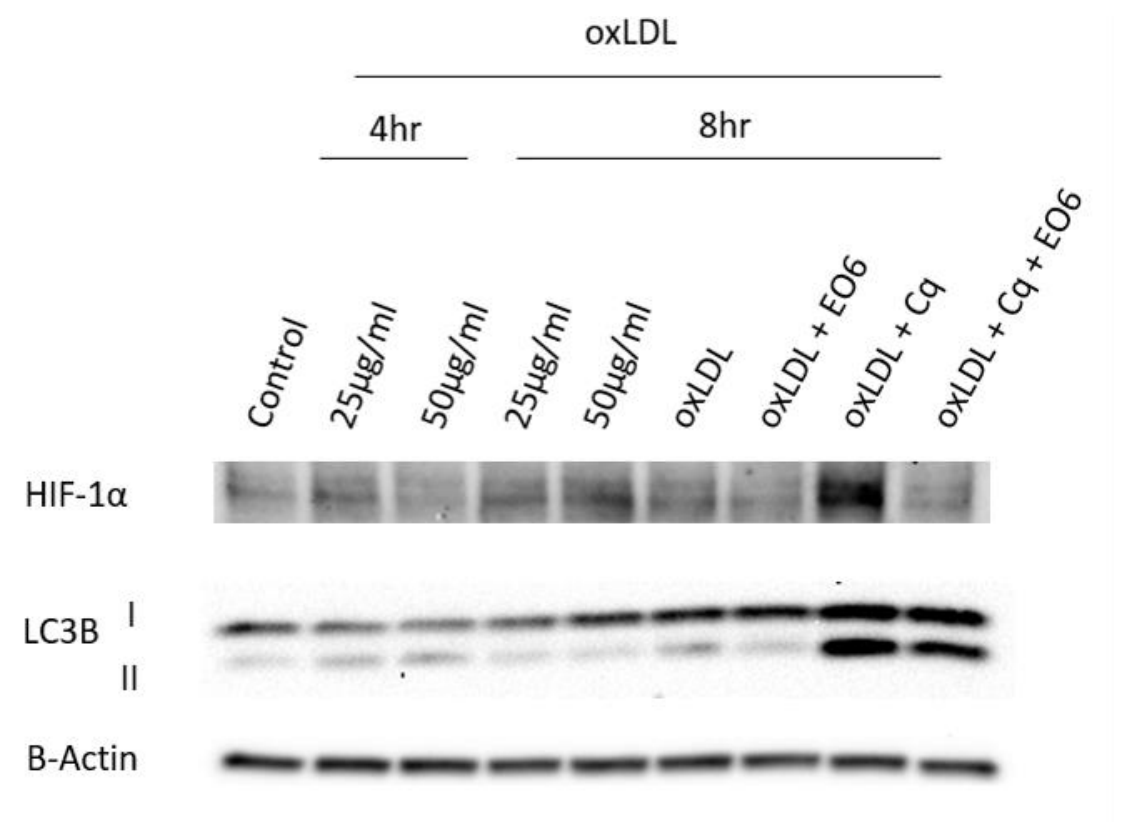

Supplemental Figure 1 OxLDL and EO6 affect HIF-1 $\alpha$ and LC3B protein expression in KLM-1. A representative westernblot of HIF-1 $\alpha$ and LC3B protein levels. KLM-1 cells were treated with oxLDL ( 25 or $50 \mu \mathrm{g} / \mathrm{ml})$ for $4 \mathrm{hr}$ or $8 \mathrm{hr}$. Additionally, KLM-1 cells were treated with oxLDL $(50 \mu \mathrm{g} / \mathrm{ml})$ or oxLDL $(50 \mu \mathrm{g} / \mathrm{ml})+$ EO6 $(14 \mu \mathrm{g} / \mathrm{ml})$ in the presence or absence of chloroquine $(20 \mu \mathrm{M})$ for $8 \mathrm{hrs}$. 
Chapter 6 


\section{Chapter 7}

Pneumococcal immunization reduces tumor burden and growth in a NASH-HCC model: preliminary data from a pilot study 


\section{Chapter 8}

General Discussion 


\section{Chapter 8}

\section{Chapter 8 General Discussion}

\section{Novel findings of this thesis}

With dysregulated lipid metabolism being among the top risk factors in a plethora of diseases such as NASH, NPC1 and cancer, lots of research has been done in recent years targeting lipids as a prevention or treatment strategy. While oxLDL is known to be involved in these diseases many of the underlying mechanisms are still not yet fully understood and in the case of cancer treatment not sufficiently explored. Therefore, in this thesis we explored the role of oxidized lipids with a primary focus on oxLDL in the pathophysiology of metabolic diseases such as NASH, NPC1 and cancer. In addition, we investigated the possibility to counteract the detrimental oxLDL-induced effects using specific antioxLDL antibodies. The main findings of this thesis are listed below.

1. OxLDL contributes to lysosomal lipid-induced hepatic inflammation. (Chapter 2)

2. Increasing anti-oxLDL IgM autoantibodies ameliorates oxLDL induced inflammation in vitro. (Chapter 2)

3. Inhibition of the proteolytic function of the lysosomal enzyme CTSD reduces inflammation in oxLDL-loaded BMDMs. (Chapter 3)

4. OxLDL stabilizes HIF1-a under normoxic conditions in pancreatic cancer cell line KLM-

\section{1. (Chapter 6)}

5. OxLDL induces autophagy in pancreatic cancer cell line KLM-1. (Chapter 6)

6. OxLDL induces a metabolic shift towards glycolysis in pancreatic cancer cell line KLM1. (Chapter 6)

7. Anti-oxLDL antibodies prevent oxLDL activation of HIF1-a and autophagy in pancreatic cell line KLM-1. (Chapter 6)

8. Anti-oxLDL immunization reduces tumor burden and growth rate in murine NASHderived HCC. (Chapter 7)

9. Cholesterol derivative $27 \mathrm{HC}$ affects inflammation differently in males versus females. (Chapter 4)

10. Differences between males and females may be explained due to differential expression patterns of estrogen receptor subtypes. (Chapter 4)

In the present thesis, strong links between oxLDL with various aspects of metabolic diseases have been disclosed through both cellular as well as preclinical and clinical studies, with several open questions that require further exploration. In the current chapter we bring together the results of the chapters to discuss the outlook on the future potential of targeting oxLDL in metabolic diseases. 


\section{Chapter 8}

\section{Involvement of oxLDL in disease}

While oxLDL is long known to be a driver of disease particularly in metabolic diseases such as NASH, atherosclerosis as well as cancer, the mechanisms through which it does so remains poorly understood. OxLDL induces lysosomal dysfunction in part due to its resistance to lysosomal esterases (1). This effect further leads to impairment of lysosomal trafficking, maturation and fusion $(2,3)$; increased lysosomal $\mathrm{pH}$ (4); triggering of various downstream inflammatory pathways such as formation of the NLRP3 inflammasome (5) and also direct damage to lysosomal membranes (6,7). In chapter 2, we demonstrated for the first time that lysosomal accumulation of oxidized lipids in blood-derived macrophages is a key trigger of hepatic inflammation using NPC1 mice as a severe model for NASH. This data is in line with studies within the atherosclerosis field, where oxLDL is thought to be one on the main drivers of foam cell formation \& plaque inflammation through lipid build up (8-10). While hepatic steatosis is a prerequisite for NASH, it is still unknown what triggers hepatic inflammation to progress from simple steatosis to NASH. With oxLDL now shown to induce hepatic inflammation, it could mean that oxLDL may also be a possible factor contributing to the process of initiation of NASH. Furthermore, we recently identified lysosomal enzyme cathepsin D to be elevated in the plasma of NASH patients and correlated to increased systemic inflammation (11). OxLDL, through impairment of proper lysosomal function, could be responsible for the release of cathepsin D into the plasma, which in turn may play a role in systemic inflammation as demonstrated in chapter 3. There, a link between oxLDL and inflammation is suggested to be in part modulated by cathepsin D as its inhibition through the use of pepstatin decreases inflammation.

Ample literature shows oxLDL also as a driver for cancer through a variety of mechanisms as summarized in chapter 5. Also, in this thesis, we explored the effect of oxLDL on metabolism in cancer cells, and as reported in chapter 6, we found that oxLDL affects pancreatic cancer cell metabolism driving these cells to have a phenotype associated with higher survival. This metabolic phenotype has also been reported in macrophages and strongly resembles the Warburg effect (12). It is widely known that cancer cells often times resort to the utilization of glycolysis over the regular oxidative phosphorylation despite the presence of adequate levels of oxygen (13). Since its discovery in 1924, this phenomenon has been an unresolved puzzle with several hypothesis suggested. With lipids playing an important role in cancer and now oxLDL shown to induce a metabolic profile similar to that of the Warburg effect, the answer to this longstanding mystery could possibly be tied to oxidized lipids and their effects on mitochondrial function and HIF-1 $\alpha$ stabilization. modulate autophagy in order to secure additional energy during metabolic stress responses such as hypoxia.

In this thesis, we have reaffirmed the heterogeneity of the involvement of oxLDL in several diseases. The fact that oxLDL is involved in these many aspects of disease is remarkable in its own right and 


\section{Chapter 8}

reinforces the importance of proper functioning of cholesterol metabolism pathways. Though much is still unknown on how exactly oxidized lipids e.g. oxLDL lead to disease, we have elucidated a small part of that process.

\section{oxLDL as a therapeutic target}

Complementary to the findings related to oxLDL as a driver of metabolic disease, this thesis also establishes the promising possibility using oxidized lipids as therapeutics or of targeting oxLDL through the use of antibodies to either revert or lessen the impact of lysosomal dysfunction mediated inflammation.

OxLDL is loaded with 7-ketosterol, which through Cyp27a in the cell is converted into the bile acid precursor $27 \mathrm{HC}$ (14). In recent years the therapeutic potential of $27 \mathrm{HC}$ has been explored for its ability to reduce inflammation. As reported in chapter 4, we noted that while $27 \mathrm{HC}$ does in fact have antiinflammatory properties as also reported by others, it does not have this effect in all patients, with sex being the determining factor for outcome. While it is known that there are differences in disease phenotype of responses to treatment based on gender, the mechanisms for these observed differences have not yet been elucidated. As discussed in chapter 4, there are discrepancies in response between men and women when it comes to for instance the usage of $27 \mathrm{HC}$ as a therapeutic agent. It has also been reported that men and women show differences in outcomes in response to chemotherapy. In chapter 4 we bring forth preliminary evidence, which suggests that differences between men and women could in part be due to different expression levels of the ERs in the same tissue type. As many genes have regulatory elements managed by the ER, it may be one of the possible pathways that dictate differences between cellular function of men and women. With personalized therapy being on the rise in recent years, it may very well be that sex would also be an important criterion to consider when diagnosing or recommending treatment.

As shown in chapters $2 \& \mathbf{6}$, antibodies against oxLDL show promising therapeutic potential by reducing the adverse effects of oxLDL on BMDMs and pancreatic cancer cells. Additionally, these antibodies have also been explored in the context of atherosclerosis as well as NPC1 where it showed to ameliorate the disease $(15,16)$. Similarly, though the results are preliminary (due to the Covid-19 situation and related measures) and the study still ongoing, in chapter 7 anti-oxLDL immunization strategies is shown to also ameliorate HCC as evidenced by reduced tumor burden and growth. While the exact mechanism on how these antibodies mediate the effects of oxLDL is not fully understood, sequestration or altered processing of oxLDL, the potential for its therapeutic use is undeniable and therefore further studies are currently underway to further evaluate the usefulness of utilizing these antibodies as a therapeutic means to combat the metabolic effects of oxLDL. As alluded to in chapter 3 part of the effect of oxLDL on inflammation may be mediated through cathepsin D and as summarized 


\section{Chapter 8}

in chapter 5 oxLDL receptors also play a noteworthy role in not only the uptake of oxLDL but also cell signaling. Based on these observations it may also be worthwhile targeting these downstream effectors and receptors of oxLDL using specific inhibitors as an alternative or complementary to oxLDL antibodies.

In summary, this thesis provides new insights into the depths of the involvement of oxLDL in several pathologies as alludes to possible mechanisms related to this involvement. Furthermore, this thesis addresses a means of therapeutically targeting oxLDL through the use of antibodies. Additionally, our findings also suggest the effect of sex on disease and treatment outcome. Taken together, our findings provide valuable mechanistic evidence of the involvement of oxLDL in disease and future research should endeavor to further elucidate these mechanisms particularly in the area of cancer therapy efficacy. 


\section{Chapter 8}

\section{References}

1. Vrieling, F., Wilson, L., Rensen, P. C. N., Walzl, G., Ottenhoff, T. H. M., and Joosten, S. A. (2019) Oxidized low-density lipoprotein (oxLDL) supports Mycobacterium tuberculosis survival in macrophages by inducing lysosomal dysfunction. PLoS Pathog 15, e1007724e1007724

2. Huynh, K. K., Gershenzon, E., and Grinstein, S. (2008) Cholesterol accumulation by macrophages impairs phagosome maturation. J Biol Chem 283, 35745-35755

3. Shen, D., Wang, X., Li, X., Zhang, X., Yao, Z., Dibble, S., Dong, X. P., Yu, T., Lieberman, A. P., Showalter, H. D., and Xu, H. (2012) Lipid storage disorders block lysosomal trafficking by inhibiting a TRP channel and lysosomal calcium release. Nat Commun 3, 731

4. Emanuel, R., Sergin, I., Bhattacharya, S., Turner, J., Epelman, S., Settembre, C., Diwan, A., Ballabio, A., and Razani, B. (2014) Induction of lysosomal biogenesis in atherosclerotic macrophages can rescue lipid-induced lysosomal dysfunction and downstream sequelae. Arterioscler Thromb Vasc Biol 34, 1942-1952

5. Duewell, P., Kono, H., Rayner, K. J., Sirois, C. M., Vladimer, G., Bauernfeind, F. G., Abela, G. S., Franchi, L., Nuñez, G., Schnurr, M., Espevik, T., Lien, E., Fitzgerald, K. A., Rock, K. L., Moore, K. J., Wright, S. D., Hornung, V., and Latz, E. (2010) NLRP3 inflammasomes are required for atherogenesis and activated by cholesterol crystals. Nature 464, 1357-1361

6. Li, W., Yuan, X. M., Olsson, A. G., and Brunk, U. T. (1998) Uptake of oxidized LDL by macrophages results in partial lysosomal enzyme inactivation and relocation. Arterioscler Thromb Vasc Biol 18, 177-184

7. Li, W., Dalen, H., Eaton, J. W., and Yuan, X. M. (2001) Apoptotic death of inflammatory cells in human atheroma. Arterioscler Thromb Vasc Biol 21, 1124-1130

8. Obermayer, G., Afonyushkin, T., and Binder, C. J. (2018) Oxidized low-density lipoprotein in inflammation-driven thrombosis. J Thromb Haemost 16, 418-428

9. Rhoads, J. P., and Major, A. S. (2018) How Oxidized Low-Density Lipoprotein Activates Inflammatory Responses. Crit Rev Immunol 38, 333-342

10. Luo, Y., Duan, H., Qian, Y., Feng, L., Wu, Z., Wang, F., Feng, J., Yang, D., Qin, Z., and Yan, X. (2017) Macrophagic CD146 promotes foam cell formation and retention during atherosclerosis. Cell Research 27, 352-372 


\section{Chapter 8}

11. Walenbergh, S. M. A., Houben, T., Rensen, S. S., Bieghs, V., Hendrikx, T., van Gorp, P. J., Oligschlaeger, Y., Jeurissen, M. L. J., Gijbels, M. J. J., Buurman, W. A., Vreugdenhil, A. C. E., Greve, J. W. M., Plat, J., Hofker, M. H., Kalhan, S., Pihlajamäki, J., Lindsey, P., Koek, G. H., and Shiri-Sverdlov, R. (2016) Plasma cathepsin D correlates with histological classifications of fatty liver disease in adults and responds to intervention. Sci Rep 6, 3827838278

12. Shatrov, V. A., Sumbayev, V. V., Zhou, J., and Brüne, B. (2003) Oxidized low-density lipoprotein (oxLDL) triggers hypoxia-inducible factor-1alpha (HIF-1alpha) accumulation via redox-dependent mechanisms. Blood 101, 4847-4849

13. López-Lázaro, M. (2008) The warburg effect: why and how do cancer cells activate glycolysis in the presence of oxygen? Anticancer Agents Med Chem 8, 305-312

14. Brown, A. J., Mander, E. L., Gelissen, I. C., Kritharides, L., Dean, R. T., and Jessup, W. (2000) Cholesterol and oxysterol metabolism and subcellular distribution in macrophage foam cells. Accumulation of oxidized esters in lysosomes. J Lipid Res 41, 226-237

15. Binder, C. J., Horkko, S., Dewan, A., Chang, M. K., Kieu, E. P., Goodyear, C. S., Shaw, P. X., Palinski, W., Witztum, J. L., and Silverman, G. J. (2003) Pneumococcal vaccination decreases atherosclerotic lesion formation: molecular mimicry between Streptococcus pneumoniae and oxidized LDL. Nat Med 9, 736-743

16. Houben, T., Magro dos Reis, I., Oligschlaeger, Y., Steinbusch, H., Gijbels, M. J. J., Hendrikx, T., Binder, C. J., Cassiman, D., Westerterp, M., Prickaerts, J., and Shiri-Sverdlov, R. (2019) Pneumococcal Immunization Reduces Neurological and Hepatic Symptoms in a Mouse Model for Niemann-Pick Type C1 Disease. Frontiers in Immunology 9 
Chapter 8 
Appendices 


\section{Appendices}

\section{Summary}

The main focus of this thesis was to elucidate the role of oxidized lipids with a primary focus on oxLDL in the pathophysiology of metabolic diseases such as NASH, NPC1 and cancer. A variety of in vitro, in vivo and clinical study methods were employed to assess the disease progression markers affected by oxLDL or derivatives thereof such as inflammation, metabolism and response to treatment. In addition, we also sought to validate the use of antibodies against oxLDL as a promising readily-available therapeutic tool to prevent the detrimental effects of oxLDL in cancer.

Chapter 1 provides a global overview of lipids, in particular oxidized lipids, lysosomal enzymes such as cathepsin $\mathrm{D}$ and the role they play in metabolic diseases

Chapter 2 explores the direct effects of oxLDL on the inflammatory status of macrophages, which are thought to be the main drivers of inflammation in diseases featuring dyslipidemia, as well as verifying the ability of anti-oxLDL antibodies to prevent these effects. Here OxLDL was found to contribute to lysosomal lipid-induced hepatic inflammation and increasing anti-oxLDL IgM autoantibodies ameliorates oxLDL induced inflammation in vitro.

Chapter 3 evaluates the effects of CTSD inhibition on the oxLDL mediated modulation of inflammation in macrophages in order to explore its therapeutic viability. The findings demonstrated that inhibition of the proteolytic function of the lysosomal enzyme CTSD reduced inflammation in oxLDL-loaded BMDMs.

Chapter 4 covers a novel attribute of an oxidized lipid (27-hydroxycholesterol) with promising lipid lowering therapeutic potential that shows different effects in men versus women. Here it was observed that cholesterol derivative $27 \mathrm{HC}$ affects inflammation differently in males versus females. These differences between males and females are thought to be due to inherent differential expression patterns of the estrogen receptor subtypes between the two sexes.

Chapter 5 Reveals the current state of our general understanding of the effects of oxLDl in cancer

Chapter 6 aimed to further elucidate the metabolic effects of oxLDL in cancer. Here it was found that oxLDL caused a metabolic shift towards glycolysis, stabilized HIF1-a under normoxic conditions and induced autophagy in pancreatic cancer cell line KLM-1. In addition, anti-oxLDL antibodies prevented the oxLDL mediated activation of HIF1-a and autophagy.

Chapter 7 showcases preliminary results of an ongoing in vivo experiment which looked into the effects of pneumococcal immunization in a NASH-HCC mice model. Here it was uncovered that antioxLDL immunization using heat-inactivated pneumococci reduces tumor burden and growth rate in murine NASH-derived HCC. 


\section{Appendices}

Finally, chapter 8 discusses the overall conclusions of this thesis and addresses open questions that can be further investigated in future research in this context. 


\section{Appendices}

\section{Valorization}

\section{Social and clinical relevance}

The metabolic syndrome and cancer are among the top ten causes of deaths in upper middle-income and high-income countries to date placing high socioeconomic burdens on the developed world making them top world health concerns. NAFLD is the hepatic component of the metabolic syndrome. Currently, the prevalence of NAFLD is estimated to be $25 \%$ world-wide, predominantly in the West, with NASH making up 5\% of the world population $(1,2)$. Similarly, cancer is among diseases with the highest prevalence worldwide tallying 18 million new cases in 2018 alone (3). Clinicians face major challenges in diagnosing and treating NASH as early non-invasive testing is unavailable and the exact underlying mechanisms triggering hepatic inflammation are still largely unknown making liver transplantation the only effective treatment for advanced liver disorders stemming from NASH (4). Because of this, there is currently an enormous demand for novel more effective early non-invasive diagnostic and therapeutic tools for NASH. Also, cancer therapy suffers from major therapy-limiting factors such as the occurrence of therapy resistance, a persisting issue despite significant advances of (neo-)adjuvant chemotherapy and radiotherapy treatment protocols. Although these improvements have undoubtedly led to improved short-term cancer outcomes and reduced tumor burden, long-term survival and tumor eradication remain disappointing even though major progress in understanding of the molecular basis of treatment response has been acquired in recent years. In order to improve long-term outcomes, it is important to establish complementary intervention strategies that sensitize tumors to current therapies to increase the therapeutic ratio. As previously discussed in this thesis, there are striking similarities between metabolic derangements in cancer and diseases related to the metabolic syndrome e.g. atherosclerosis and NASH (5). Subject of this thesis in particular is the similarity of broad involvement of oxLDL in the pathologies of NASH and cancer. While oxLDL is long known to be a driver of disease particularly in metabolic diseases such as NASH, atherosclerosis as well as cancer, the mechanisms through which it does so remains poorly understood. By further exploring the role of oxLDL and related components in the pathophysiology of these metabolic diseases this thesis showcases the therapeutic potential of a non-invasive method of counteracting the detrimental oxLDLinduced effects through use of specific anti-oxLDL antibody strategies. Successful translation and implementation of these findings in the future into the clinic would potentially lead to improvement of quality of life for NASH and cancer patients and reduction of treatment cost. 


\section{Appendices}

\section{Novelty of the concept}

In this thesis we investigated the mechanism through which oxLDL contributes to inflammation in NASH and the potential of oxLDL targeting in this setting. Hence this thesis provides new mechanistic insights of direct effect of oxLDL and the potential of directly targeting oxLDL in the context of NASH. Furthermore, while oxLDL has been extensively studied for its effect on cancer development and progression there has been no attempts to ascertain the benefits of targeting oxLDL directly in the context of cancer and little to no research on the effect of oxLDL on cancer therapy (3). This thesis expanded on the prospect of targeting oxLDL directly and suggests that this approach may be promising for future consideration. Finally, in recent years many studies have suggested that the future of therapy should be developed in the form of personalized treatment. This thesis further demonstrates the need for this consideration and that sex be a strong determining factor for this based on the duality of response to $27 \mathrm{HC}$ in men and women. In addition, this thesis also suggests a novel mechanism where differences ER receptor expression between men and women may possibly be key to explaining the differences in response to treatment between men and women.

\section{Future perspectives and potential application}

The studies described in this thesis facilitate the clinical translation of using oxLDL antibodies in NASH and cancer treatment. The fact that antibodies against oxLDL are naturally present in the human body makes them particularly attractive targets for intervention. And as there are already existing vaccines and new ones being developed to induce immunity against pneumococci infection which do not lead to any side effects, it further enables acceleration of the process of repurposing this vaccine for use in NASH and cancer clinical trials to target oxLDL. In the meantime, before this point of transition is reached, research is currently ongoing to further strengthen the basis of this approach. In particular the effectiveness of immunization in restoring treatment sensitivity in vivo will be determined. Additionally, the mechanism through which ER receptor expression is thought to result in variances of response between men and women still needs to be fleshed out and the effect of this verified across other diseases that show clear disparities between the sexes. This will facilitate future endeavors down the road to personalized treatment but also help with understanding mechanism of diseases that show discrepancies in pathophysiology between men and women. 


\section{Appendices}

\section{References}

1. Araújo, A. R., Rosso, N., Bedogni, G., Tiribelli, C., and Bellentani, S. (2018) Global epidemiology of non-alcoholic fatty liver disease/non-alcoholic steatohepatitis: What we need in the future. Liver Int 38 Suppl 1, 47-51

2. Povsic, M., Wong, O. Y., Perry, R., and Bottomley, J. (2019) A Structured Literature Review of the Epidemiology and Disease Burden of Non-Alcoholic Steatohepatitis (NASH). Adv Ther $36,1574-1594$

3. Bitorina, A. V., Oligschlaeger, Y., Shiri-Sverdlov, R., and Theys, J. (2019) Low profile high value target: The role of OxLDL in cancer. Biochim Biophys Acta Mol Cell Biol Lipids 1864, 158518

4. Wong, R. J., Aguilar, M., Cheung, R., Perumpail, R. B., Harrison, S. A., Younossi, Z. M., and Ahmed, A. (2015) Nonalcoholic steatohepatitis is the second leading etiology of liver disease among adults awaiting liver transplantation in the United States. Gastroenterology 148, 547555

5. Hirsch, H. A., Iliopoulos, D., Joshi, A., Zhang, Y., Jaeger, S. A., Bulyk, M., Tsichlis, P. N., Shirley Liu, X., and Struhl, K. (2010) A transcriptional signature and common gene networks link cancer with lipid metabolism and diverse human diseases. Cancer Cell 17, 348-361 